\title{
Towards a Precision Measurement of Parity-Violating e-p Elastic Scattering at Low Momentum Transfer
}

\author{
by
}

Jie Pan

\begin{abstract}
A Thesis submitted to the Faculty of Graduate Studies of
The University of Manitoba

in partial fulfillment of the requirements of the degree of
\end{abstract}

DOCTOR OF PHILOSOPHY

\author{
Department of Physics and Astronomy \\ University of Manitoba \\ Winnipeg
}

Copyright (C) 2012 by Jie Pan 
This work is dedicated

to my family. 


\section{Acknowledgments}

I have been indebted to numerous individuals. Without their support and guidance, this dissertation would not have been possible. It is a pleasure to convey my gratitude to them all in my humble acknowledgement.

Foremost, I would like to express my sincere gratitude to my thesis advisor, Prof. Jeff Martin, for the continuous support of my $\mathrm{Ph} . \mathrm{D}$. study and research, for his patience, as well as his immense knowledge. His guidance helped me in all the time of research and writing of this thesis. From the lab experiences to the critical comments on my writing, I am truly grateful in the assistance offered by him.

Besides my advisor, many thanks go in particular to the rest of my Ph.D. advisory committee for their valuable advice. I am much indebted to Prof. Shelley Page for offering me the opportunity to work on the Q-weak experiment. I am grateful for her steady encouragement and support in various ways, especially for spending her precious time on reading the countless revisions of my thesis and giving her insightful comments. I have also benefited by advice and guidance from Prof. James Birchall, who kindly grants me his time on reading and providing his constructive suggestions. My sincere thanks also goes to Prof. Douglas Goltz, for providing me encouragement and valuable advice. I convey special acknowledgement to the late Prof. Randy Kobes, not only for his suggestion on my research project, but also for the knowledge on Quantum Field Theory learned from him.

I am grateful for the mentorship and assistance from outstanding individuals, including Prof. David Armstrong, Dr. David Mack and Dr. David Gaskell and Dr. Gregory Smith. My research 
project bears on imprint of many peoples, I wish to express my gratitude to the members of the University of Manitoba Subatomic Group, including Prof. Willem T.H. van Oers, Prof. Michael Gericke, Dr. Willie Falk, Dr. Desmond Ramsay, Dr. Lawrence Lee, Dr. Charles Davis, Dr. Rob Mahurin, Dr. Vladas Tvaskis and Scott MacEwan, and my Q-weak collaborators, including Dr. Paul King, Dr. Wouter Deconinck, Dr. Jeong Han Lee, Dr. Anna Micherdzinska, as well as graduate students, including Siyuan Yang, John Leacock, Katherine Myers, Buddhini Waidyawansa, Rakitha Beminiwattha, Joshua Hoskins, Nuruzzaman and Narayan Amrendra. Thanks to these individuals who rendered their help in carrying out my work during the period of this research project. I'd like to give special thanks to James Dowd and Rob Mahurun who took over the scanner responsibility in Q-weak Run II, and also to NSERC Canada for funding my study, research and the experiment. Last but not least, I wish to avail myself of this opportunity to express a sense of gratitude and love to my family and friends for their understanding and support. 


\section{Abstract}

The goal of the Q-weak experiment is to make a measurement of the proton's weak charge $Q_{W}^{p}=$ $1-4 \sin ^{2} \theta_{W}$ to an accuracy of $\sim 4 \%$. This would represent $\mathrm{a} \sim 0.3 \%$ determination of the weak mixing angle $\sin ^{2} \theta_{W}$ at low energy. The measurement may be used for a precision test of the Standard Model (SM) prediction on the running of $\sin ^{2} \theta_{W}$ with energy scale. The Q-weak experiment operates at Thomas Jefferson National Accelerator Facility (Jefferson Lab). The experiment determines $Q_{W}^{p}$ by measuring the parity violating asymmetry in elastic electron-proton scattering at low momentum transfer $Q^{2}=0.026(\mathrm{GeV} / \mathrm{c})^{2}$ and forward angles $\left(\sim 8^{\circ}\right)$. The anticipated size of the asymmetry, based on the SM, is about 230 parts per billion (ppb). With the proposed accuracy, the experiment may probe new physics beyond Standard Model at the TeV scale.

This thesis focuses on my contributions to the experiment, including track reconstruction for momentum transfer determination of the scattering process, and the focal plane scanner, a detector I designed and built to measure the flux profile of scattered electrons on the focal plane of the Q-weak spectrometer to assist in the extrapolation of low beam current tracking results to high beam current. Preliminary results from the commissioning and the first run period of the Q-weak experiment are reported and discussed. 


\section{Table of Contents}

Nomenclature $\quad$ xiii

1 Theory and Motivation 1

1.1 Introduction . . . . . . . . . . . . . . . 1

1.2 Theoretical Background and Motivation . . . . . . . . . . . . 2

1.2.1 Electroweak Standard Model and the Weak Mixing Angle . . . . . . . . 2

1.2.2 Running of the Weak Mixing Angle . . . . . . . . . . . . 6

1.3 Methodology of Measuring $\mathrm{Q}_{\mathrm{W}}^{\mathrm{p}} \ldots \ldots \ldots \ldots \ldots$

1.3.1 e-N Elastic Scattering Kinematics . . . . . . . . . . . . . . . 8

1.3.2 Scattering Amplitude and Cross-section . . . . . . . . . . . . . . . . . . . 10

1.3.3 Parity-Violating Asymmetry . . . . . . . . . . . . . . . . . . . . . 14

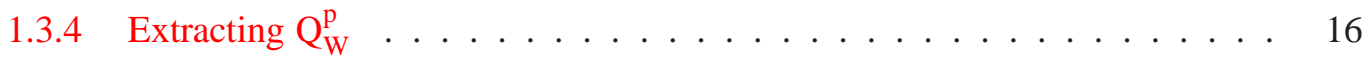

1.3.5 Electroweak Radiative Corrections . . . . . . . . . . . . . . . . . . . . . 18

1.4 Constraints on the Standard Model Parameters and New Physics . . . . . . . . . 22

1.4.1 Constraints on Quark Vector Couplings . . . . . . . . . . . . . . . . . . 22

1.4.2 Example of Model-independent Constraints . . . . . . . . . . . . . 23

1.4.3 Model-dependent Constraints . . . . . . . . . . . . . . 25

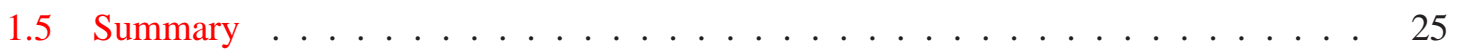

2 The Q-weak Experiment 26

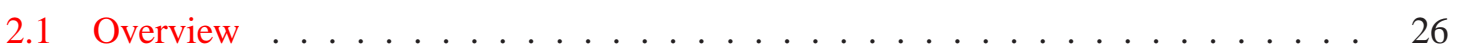

2.2 The Polarized Electron Beam . . . . . . . . . . . . . . . . . . . . . . . . . 29

2.2.1 CEBAF Accelerator . . . . . . . . . . . . . . . . . . . 29

2.2.2 The Polarized Electron Source . . . . . . . . . . . . . . . . . 30

2.2 .3 Beam Polarimetry . . . . . . . . . . . . . . . 33

2.2 .4 Beam Monitors . . . . . . . . . . . . . . . . . . . . 38

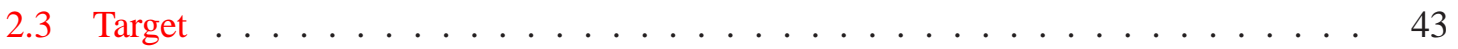


2.4 Spectrometer System . . . . . . . . . . . . . . . . . . . . . . . 45

2.4.1 Collimator and Shielding Wall . . . . . . . . . . . . . . . . . 45

2.4.2 Toroidal Magnet . . . . . . . . . . . . . . . . . . 46

2.4 .3 Main Detectors . . . . . . . . . . . . . . . . . . . . . . 47

2.4.4 Tracking System . . . . . . . . . . . . . . . . . . . . . . . . . 49

2.4.5 Luminosity Monitors . . . . . . . . . . . . . . . . . . . . 54

2.5 Data Acquisition . . . . . . . . . . . . . . . . . . 55

2.6 Data Analyzer . . . . . . . . . . . . . . . . . . . . . . . 57

3 Electron Flux Profile Scanner $\quad 59$

3.1 Motivation . . . . . . . . . . . . . . . . . . . . . . . 59

3.2 E158 Scanner Review . . . . . . . . . . . . . . . . . . . . . . 60

3.3 Tailoring the E158 Scanner Design for Q-weak . . . . . . . . . . . . . 62

3.4 Basic Q-weak Scanner Design . . . . . . . . . . . . . . . . . 64

3.4 .1 Detector . . . . . . . . . . . . . . . . . . 64

3.4.2 Linear Motion Assembly . . . . . . . . . . . . . . . . . 66

3.5 Monte Carlo Simulations of the Scanner Detector and Backgrounds _ . . . . . . 68

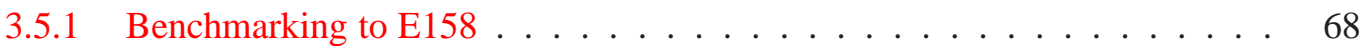

3.5.2 Geometry and Material Studies . . . . . . . . . . . . . 70

3.5 .3 Signal . . . . . . . . . . . . . . . . . . 71

3.5.4 Light-guide Associated Backgrounds _ . . . . . . . . . . . . . 72

3.6 Cosmic Ray Testing of Prototype Detectors . . . . . . . . . . . . . . . . 73

3.7 TRIUMF Beam Tests . . . . . . . . . . . . . . . . . . . . . . . . . . 74

3.7.1 Signal and Comparison to Simulations . . . . . . . . . . . . . . . . 74

3.7 .2 Background Light Yield . . . . . . . . . . . . . . . . 78

3.8 Monte Carlo Simulations of Scan Efficiency . . . . . . . . . . . . . . . . 78

3.9 Final Detector Design and Construction . . . . . . . . . . . . . . 85

3.9 .1 Radiator . . . . . . . . . . . . . . . . . . . . 85

3.9 .2 Light Guides . . . . . . . . . . . . . . . . . . . . . . . . . . . . . . . 86

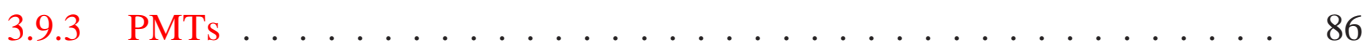

3.9.4 Detector Housing and Mechanics _ . . . . . . . . . . . . . . . . . 88

3.10 Linear Motion System ． . . . . . . . . . . . . . . . . . . . 88

3.10 .1 Hardware . . . . . . . . . . . . . . . . . . . . . 88

3.10 .2 Software and GUI . . . . . . . . . . . . . . . . . . 91

3.10.3 Mounting of Detector on the 2D Motion System . . . . . . . . . . . . 92

3.10 .4 Mounting in Q-weak . . . . . . . . . . . . . . . . . . . . . . 92 
3.11 Electronics and DAQ . . . . . . . . . . . . . . . . . . . . . . . 94

3.12 Installation and Commissioning . . . . . . . . . . . . . . 96

3.13 Monte Carlo Simulations of Scanner Applications . . . . . . . . . . . . . . . . 98

4 Momentum Transfer Determination $\quad 100$

4.1 Overview . . . . . . . . . . . . . . . . . . 100

4.2 Coordinate Systems and Naming Conventions . . . . . . . . . . . . . . . 102

4.3 Partial Track Finding . . . . . . . . . . . . . . . . . . . . . . . 104

4.3.1 Pattern Recognition Algorithm . . . . . . . . . . . . . . . . . . . 104

4.3.2 Parameterization of Partial Track Projections ． . . . . . . . . . . . . . . . 107

4.3.3 Partial Tracks in Three Dimensions _. . . . . . . . . . . . . . . . . . . . 109

4.4 Bridging Partial Tracks . . . . . . . . . . . . . . . . . 111

$4.4 .1 \quad$ Track Filter . . . . . . . . . . . . . . . . . . . . . . . 112

4.4.2 Tracking in the Magnetic Field . . . . . . . . . . . . . . . 113

4.4 .3 The Momentum Matrix . . . . . . . . . . . . . . . . . . . . . . . 115

4.4.4 Shooting Method . . . . . . . . . . . . . . . . . . . . 118

4.4.5 Momentum Transfer Determination . . . . . . . . . . . . . . . . . . 119

4.5 Bridging Performance Test . . . . . . . . . . . . . . . . . . . . 121

4.6 Preliminary Tracking Results . . . . . . . . . . . . . . . . 126

5 Scanner Data Analysis $\quad 131$

5.1 Focal Plane Flux Profile . . . . . . . . . . . . . . . . . . . . . . 131

5.1.1 Raw Rate Distribution . . . . . . . . . . . . . . . . . . . . . . . 132

5.1 .2 Rate Corrections . . . . . . . . . . . . . . . . . . . . 135

5.1.3 Scanner Results Discussion . . . . . . . . . . . . . . . . . 145

5.2 Momentum Transfer Extrapolation . . . . . . . . . . . . . . . . . . . . . . . . . 149

5.2.1 Calibration to Region 3 VDCs . . . . . . . . . . . . . . . . . . . . 149

5.2.2 Dimensionless Normalization _. . . . . . . . . . . . . . . . 151

5.2 .3 Contrast . . . . . . . . . . . . . . . . . . . . . . 152

5.2.4 Light-weighted $\left\langle\mathrm{Q}^{2}\right\rangle$ Extrapolation . . . . . . . . . . . . . . . . 156

5.3 Other Applications . . . . . . . . . . . . . . . . . . . . . . . . 162

5.4 Summary and Outlook . . . . . . . . . . . . . . . . . . . 167

6 Parity Data Analysis $\quad 169$

6.1 General Analysis Procedures . . . . . . . . . . . . . . . . . . . . 169

6.2 Raw Asymmetry . . . . . . . . . . . . . . . . . . . . . . 171

6.2 .1 Initial Data Processing . . . . . . . . . . . . . . . . . . . . 171 
6.2 .2 Data Selection . . . . . . . . . . . . . . . . . . . 172

6.2 .3 Raw Asymmetry Calculation . . . . . . . . . . . . . . . . . 173

6.2 .4 Data Quality in Run I . . . . . . . . . . . . . . . . . . . . . . . 176

6.3 Applying Corrections . . . . . . . . . . . . . . . . . . . . 179

6.3 .1 Linear Regression _ . . . . . . . . . . . . . . . . . . . . 180

6.3 .2 Backgrounds Correction . . . . . . . . . . . . . . . . . 181

6.3.3 Beam Polarization Correction . . . . . . . . . . . . . . . . . . . 182

6.3.4 Hadronic Structure Correction . . . . . . . . . . . . . . . . . . . . . . 184

6.4 Physics Result Extraction . . . . . . . . . . . . . . . . . . . . . . . . . 184

6.5 Preliminary Analysis Results . . . . . . . . . . . . . . . . . . . . 185

6.5.1 Helicity Correlated Beam Property Corrections . . . . . . . . . . . . . . . 186

6.5.2 Beam Polarization . . . . . . . . . . . . . . . . . . . 186

6.5 .3 Backgrounds . . . . . . . . . . . . . . . . . . . . . . 189

6.5.4 Transverse Asymmetry . . . . . . . . . . . . . . . . . . . . 190

6.5 .5 Raw Asymmetry . . . . . . . . . . . . . . . . . . 192

7 Qweak Status Summary and Conclusion 195

$\begin{array}{ll}\text { Bibliography } & 198\end{array}$

$\begin{array}{ll}\text { Appendices } & 211\end{array}$

A Engineering Drawing of the 2D Motion Assembly 211

B Scanner Run List 213

$\begin{array}{lll}\text { C My Contributions to the Qweak Experiment } & 217\end{array}$

$\begin{array}{ll}\text { D Qweak Collaboration } & 219\end{array}$

$\begin{array}{ll}\text { Index } & 224\end{array}$ 


\section{List of Figures}

1.1 Three types of fermion gauge interactions $\ldots \ldots \ldots \ldots \ldots$

1.2 Running of weak mixing angle $\ldots \ldots \ldots \ldots \ldots \ldots$

1.3 Exchange $\gamma$ and $Z$ boson between the electron and the nucleon . . . . . . . . 10

1.4 Global fit of PVES asymmetries . . . . . . . . . . . . . . . . . . . 17

1.5 Typical one-loop self-energy diagrams . . . . . . . . . . . . . . . . . 19

1.6 Typical $\gamma Z$ loop and box diagrams . . . . . . . . . . . . . . . . . . . . . . . . 19

1.7 Constraints on the quark couplings . . . . . . . . . . . . 23

1.8 Model independent mass limits . . . . . . . . . . . . . . . . . . . . . . . . 24

2.1 Q-weak 3D layout in Hall C . . . . . . . . . . . . . . . . . 27

2.2 The Q-weak apparatus . . . . . . . . . . . . . . . . . . . . . . . 28

2.3 The CEBAF accelerator . . . . . . . . . . . . . . . . . . 30

2.4 The layout of the polarized source $\ldots \ldots \ldots \ldots \ldots \ldots \ldots \ldots$

2.5 The Møller polarimeter layout . . . . . . . . . . . . . . . . . . . . . . 34

2.6 The schematic diagram of the Compton polarimeter . . . . . . . . . . . 35

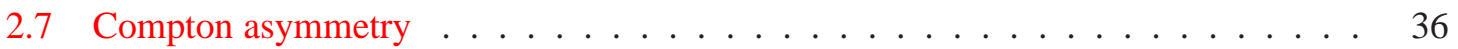

2.8 The Compton multi-strip diamond electron detector . . . . . . . . . . . . . . 37

2.9 Halo monitor position . . . . . . . . . . . . . . . . . . . 41

2.10 Two views of the Q-weak high power cryo $\mathrm{LH}_{2}$ target $\ldots \ldots \ldots \ldots$

2.11 A picture for collimator system . . . . . . . . . . . . . . . . 46

2.12 A view of QTOR . . . . . . . . . . . . . . . . . . . 47

2.13 Main detectors $\ldots \ldots \ldots \ldots \ldots \ldots \ldots$

2.14 Region 2 HDC chambers . . . . . . . . . . . . . . . . . . 51

2.15 Region 3 VDC chambers . . . . . . . . . . . . . . . . . . . . 52

2.16 A view of trigger scintillator $\ldots \ldots \ldots \ldots \ldots$

2.17 The Q-weak luminosity monitors . . . . . . . . . . . . . . . . . . . 54

2.18 Schematic diagram of the Q-weak data acquisition system $\ldots \ldots \ldots \ldots$ 
2.19 Schematic diagram of the Q-weak data paths . . . . . . . . . . . . 58

3.1 Beam's eye view of the E158 scanner system _ . . . . . . . . . . . . 61

3.2 The radial rate distribution acquired by E158 scanner detectors . . . . . . . . . . 61

3.3 The schematic layout of an E158 scanner detector . . . . . . . . . . . . . . . 62

3.4 Schematics of the Q-weak scanner detector . . . . . . . . . . . . . . . 64

3.5 Čerenkov light wavelength distribution . . . . . . . . . . . . . . . 66

3.6 CAD model of scanner detector mounted in bottom octant . . . . . . . . . . 67

3.7 Benchmarking the Q-weak scanner simulation against E158 f . . . . . . . 68

3.8 Visualization of the scanner simulation for benchmarking to E158 . . . . . . . . 69

3.9 Optimizing the cone opening angle of light guide . . . . . . . . . . . 71

3.10 Scanner detector cosmic ray test . . . . . . . . . . . . . . 73

3.11 TRIUMF beam test setup . . . . . . . . . . . . . . . . . . 75

3.12 TOF spectrum for particle identification $\ldots \ldots \ldots \ldots$

3.13 Comparisons of scanner pulse height spectra . . . . . . . . . . . . . . . 77

3.14 Scanner backgrounds in air-core light guides . . . . . . . . . . . . . . . . . 79

3.15 The scanner simulation visualization . . . . . . . . . . . . . . 80

3.16 Simulated scanner light yield spectra . . . . . . . . . . . . . . . . . 80

3.17 Simulated scanner detector efficiency map . . . . . . . . . . . . . . . 81

3.18 Simulated scan efficiency versus threshold setting . . . . . . . . . . . . . 83

3.19 Simulated scanner rate map . . . . . . . . . . . . . . . . . . . . . 84

3.20 Photo of cutting quartz radiator $\ldots \ldots \ldots \ldots \ldots$

3.21 Typical spectral characteristics of XP2268 PMT _ . . . . . . . . . . . . . 87

3.22 The inner structure of the scanner detector housing . . . . . . . . . . . . 88

3.23 Pictures of the motion control and position recording hardware . . . . . . . . . 89

3.24 The scanner GUI for 2D motion control . . . . . . . . . . . . . . . . . . 91

3.25 Extension brackets for the mounting scanner detector in different z-planes . . . . . 93

3.26 Block diagram of the scanner electronics chain . . . . . . . . . . . . . . . 95

3.27 The as-installed scanner detector in Q-weak . . . . . . . . . . . . . . . . 96

3.28 Vertical S-scan pattern . . . . . . . . . . . . . . . . . . . . . . 97

3.29 A rate distribution measured by the scanner . . . . . . . . . . . . . . . . . 98

3.30 Simulated rate distribution with varying BFIL . . . . . . . . . . . . . . . . 99

3.31 Simulated position variations versus BFIL variations . . . . . . . . . . . . . . 99

4.1 Q-weak coordinate systems . . . . . . . . . . . . . . . . . . . . . . . . 102

4.2 The Region 2 xuv-coordinates . . . . . . . . . . . . . . . . . . . . . . . 104

4.3 The Region $3 u v$-coordinates . . . . . . . . . . . . . . . . . . . . 104 
4.4 Side views of the Region 2 and Region 3 wire planes in simulation . . . . . . . . 105

4.5 Region 2 HDC bit pattern in four wire planes . . . . . . . . . . . . . 106

4.6 Region 3 HDC bit pattern in a single wire plane . . . . . . . . . . . . . . 106

4.7 Partial track in three dimensions . . . . . . . . . . . . . . . . . . 111

4.8 Flow chart of partial track bridging . . . . . . . . . . . . . . . . . . . 112

4.9 The shape of QTOR magnetic field . . . . . . . . . . . . . . . . . . . . 114

4.10 Momentum matrix parameters . . . . . . . . . . . . . . . . . 116

4.11 Shooting method using Newton iteration . . . . . . . . . . . . . . . 118

4.12 Energy loss simulation in QweakSimG4 . . . . . . . . . . . . . . . . 120

4.13 A simulated pre-scattering energy distribution . . . . . . . . . . . . . . . . 121

4.14 The simulated tracks in QweakSimG4 . . . . . . . . . . . . . . . . . 122

4.15 Computing time comparison for different bridging algorithms . . . . . . . . . . . . 122

4.16 Distribution of momentum residuals for "ideal" simulation events . . . . . . . 123

4.17 Comparison of simulated and reconstructed momentum distributions . . . . . . . . 124

4.18 Distribution of momentum residuals for "realistic" simulation events . . . . . . . . 125

4.19 Partial track projections . . . . . . . . . . . . . . . . . . . . 127

4.20 Projected scattering vertices f . . . . . . . . . . . . . . . . . 128

4.21 Reconstructed scattering angle and kinetic energy . . . . . . . . . . . . 128

4.22 Distributions of events on the main detector . . . . . . . . . . . . . . 129

4.23 Reconstructed momentum transfer and light-weighted momentum transfer . . . . . 129

5.1 Raw rate maps of total coincidence and accidental coincidence . . . . . . . . . 133

5.2 Singles rate $\operatorname{map} \ldots \ldots \ldots \ldots$. . . . . . . . . . . . . . . . . . 134

5.3 Projection of the scanner singles rate maps . . . . . . . . . . . . . 135

5.4 Scanner light guides and PMTs exposed to the gamma rays . . . . . . . . . . . 136

5.5 Waveforms of typical scanner PMT output pulses . . . . . . . . . . . . . . 137

5.6 Coincidence rate distribution for run $6616 \ldots \ldots \ldots$. . . . . . . . . . 138

5.7 Measured deadtime for run $11431 \ldots \ldots \ldots$

5.8 Deadtime correction to singles rate distribution . . . . . . . . . . . . . 140

5.9 Rate map with deadtime correction . . . . . . . . . . . . . . . . . . . . . 141

5.10 Beam current map and normalized scanner rate map for run 10044 . . . . . . . . 141

5.11 Locations of left, central and right bins . . . . . . . . . . . . . . . . 142

5.12 Measured deadtime versus the discriminator pulse width settings . . . . . . . . . . 143

5.13 Singles and coincidence rate corrections . . . . . . . . . . . . . . . . 144

5.14 Normalized rate distribution in the $x$ - and $y$-directions $\ldots \ldots \ldots$. . . . . . 145

5.15 VDC track propagation onto scanner plane . . . . . . . . . . . . . . . . . . 148

5.16 Scanner single point calibration to VDC . . . . . . . . . . . . 150 
5.17 Contrast versus beam current . . . . . . . . . . . . . . . . 153

5.18 Scanner's contrast at $10 \mu \mathrm{A}$ beam current . . . . . . . . . . . . . . . . 154

5.19 Scanner's contrast at $50 \mu \mathrm{A}$ beam current . . . . . . . . . . . . . . . 154

5.20 Rates at the left, central and right bins versus beam current . . . . . . . . . 155

5.21 Light-weighted $\mathrm{Q}^{2}$ distribution extrapolated to $50 \mu \mathrm{A} \ldots \ldots \ldots \ldots \ldots$

5.22 The extrapolated light-weighted $\left\langle\mathrm{Q}^{2}\right\rangle$ at five different beam currents . . . . . . . 159

5.23 Comparison of rate and light distribution in Octant 5 and 7 . . . . . . . . . 160

5.24 Rate maps for $1 \%$ US Al solid target . . . . . . . . . . . . . . . . . 165

5.25 The comparison of the profile projections at different QTOR currents . . . . . . 166

5.26 Rate map and projection for 4\% DS Al solid target . . . . . . . . . . . . 166

5.27 Scanner charge ADC spectra . . . . . . . . . . . . . . . . . . . . 167

6.1 The flow chart of the Q-weak analysis procedures . . . . . . . . . . . . 170

6.2 Main detector asymmetries . . . . . . . . . . . . . . . . . . . 175

6.3 The raw asymmetry of the combination of all main detectors . . . . . . . . . 176

6.4 The evaluation on expected statistical uncertainty versus scheduled beam time . . . 178

6.5 The slug plot for production runs with $\mathrm{LH}_{2}$ target . . . . . . . . . . . . . . . . 179

6.6 The main detector all bar sensitivities . . . . . . . . . . . . . . . . 187

6.7 Helicity correlated beam property corrections to asymmetry . . . . . . . . . . . 187

6.8 Sum of all helicity correlated beam property corrections . . . . . . . . . . . . . 188

6.9 Beam polarization result . . . . . . . . . . . . . . . . . . . . . . 189

6.10 Transverse asymmetry measurements . . . . . . . . . . . . . . . 191

6.11 The parity-violating asymmetries for 5 Wien settings . . . . . . . . . . . 193

6.12 Total average of asymmetries for all Wien periods . . . . . . . . . . . . . . 193

A.1 Engineering drawing of Lintech 2D motion assembly . . . . . . . . . . . . 212

B.1 Normalized scanner rate map at $10 \mu \mathrm{A}$ beam current $\ldots \ldots \ldots$. . . . . . . 215

B.2 Normalized scanner rate map at $50 \mu \mathrm{A}$ beam current . . . . . . . . . . . 215

B.3 Normalized scanner rate map at $145 \mu \mathrm{A}$ beam current . . . . . . . . . . . . . . 215

B.4 Normalized scanner rate map at $150 \mu \mathrm{A}$ beam current . . . . . . . . . . . . . . 216

B.5 Normalized scanner rate map at $165 \mu \mathrm{A}$ beam current . . . . . . . . . . . . . . 216 


\section{List of Tables}

1.1 Electric and weak charges of elementary fermions . . . . . . . . . . 5

1.2 Quark weak couplings . . . . . . . . . . . . . . . . 6

1.3 Uncertainties and sources for $\mathrm{SM}$ prediction on $\mathrm{Q}_{\mathrm{W}}^{\mathrm{p}} \ldots \ldots \ldots \ldots \ldots . \ldots . \ldots 21$

$1.4 \gamma$ Z-box correction . . . . . . . . . . . . . . . . . . . . 22

2.1 The anticipated experimental uncertainties . . . . . . . . . . . . . . . 27

2.2 The summary of systematic error requirements $\ldots \ldots \ldots \ldots \ldots$

2.3 Main trigger sources and types in Q-weak . . . . . . . . . . . . . . . 57

3.1 Comparison of the simulated number of photons reaching PMT . . . . . . . 70

3.2 Quartz wrapping material comparisons . . . . . . . . . . . . . . . 78

3.3 Scan ranges upstream and downstream of the main detector . . . . . . . . . . 93

4.1 Residuals of different track parameters . . . . . . . . . . . . . . . . 125

5.1 Comparison of rate corrections at several positions . . . . . . . . . . . . . . 142

5.2 Left-right asymmetries of several distributions . . . . . . . . . . . . . . 161

5.3 Peak position and maximum rate at three different QTOR currents . . . . . . . . 164

6.1 Summary of background source and estimated contributions _ . . . . . . . 183

6.2 Summary of hadronic structure corrections . . . . . . . . . . . . . . . . . . . . 184

6.3 Summary of helicity correlated beam properties . . . . . . . . . . . . 188

6.4 Preliminary background dilution and correction results . . . . . . . . . . . . 190

6.5 Q-weak Run I asymmetry data . . . . . . . . . . . . . . . . . . . . . . 192

B.1 Scanner run list during Q-weak Run I . . . . . . . . . . . . . . . . . . . . . 214 


\section{Nomenclature}

\begin{tabular}{|c|c|}
\hline $\mathrm{ADC}$ & Analog-to-Digital Converter \\
\hline APV & Atomic Parity Violation \\
\hline $\mathrm{BCM}$ & Beam Charge Monitor \\
\hline BPM & Beam Position Monitor \\
\hline CAD & Computer-Aided Design \\
\hline CEBAF & Continuous Electron Beam Accelerator Facility \\
\hline CERN & European Organization for Nuclear Research \\
\hline CHL & Central Helium Liquefier \\
\hline CMM & Coordinate Measuring Machine \\
\hline CODA & CEBAF Online Date Acquisition \\
\hline CsI & Cesium Iodide \\
\hline CVD & Chemical Vapor Deposition \\
\hline $\mathrm{CW}$ & Continuous Wave \\
\hline DAQ & Data Acquisition \\
\hline DCCT & DC Current Transformer \\
\hline DMC & Dynamic Motion Control \\
\hline DS & Downstream \\
\hline DT & Dead-Time \\
\hline
\end{tabular}




\begin{tabular}{|c|c|}
\hline EEL & Experimental Equipment Laboratory \\
\hline EM & Electromagnetic \\
\hline EOT & End-of-Travel \\
\hline ESR & End Station Refrigerator \\
\hline FR & Fast Raster \\
\hline GEM & Gas Electron Multiplier \\
\hline GSO & Germanium Silicon Oxide \\
\hline GUI & Graphic User Interface \\
\hline HDC & Horizontal Drift Chamber \\
\hline HPC & Helicity Pockels Cell \\
\hline $\mathrm{I}-\mathrm{V}$ & Current-to-Voltage Converter \\
\hline IHWP & Insertable Half Wave Plate \\
\hline JLab & Thomas Jefferson National Laboratory \\
\hline $\mathrm{LCP}$ & Left-Handed Circularly Polarized \\
\hline LEP & Large Electron Positron Collider \\
\hline $\mathrm{LH}_{2}$ & Liquid Hydrogen \\
\hline LHC & Large Hadron Collider \\
\hline LINAC & Linear Accelerator \\
\hline LSB & Least Significant Bit \\
\hline LUMI & Luminosity Monitor \\
\hline $\mathrm{MC}$ & Monte Carlo \\
\hline MD & Main Detector \\
\hline MIT-Bates & Bates Linear Accelerator Center at Massachusetts Institute of Technology \\
\hline MPS & Macro-Pulse \\
\hline
\end{tabular}




\begin{tabular}{|c|c|}
\hline MSSM & Minimal Supersymmetric Standard Model \\
\hline MT_TIR & Master Trigger \\
\hline NIM & Nuclear Instrument \\
\hline $\mathrm{NuTeV}$ & Deep Inelastic Neutrino-Nucleus Scattering \\
\hline $\mathrm{PbWO}_{4}$ & Lead Tungstate \\
\hline $\mathrm{PC}$ & Parity-Conserving \\
\hline PDG & Particle Data Group \\
\hline $\mathrm{PE}$ & Photo-Electron \\
\hline PID & Proportional-Integral-Derivative \\
\hline PITA & Polarization Induced Transport Asymmetry \\
\hline PMT & Photo-Multiplier Tube \\
\hline PV & Parity Violation, or Parity-Violating \\
\hline $\mathrm{PVC}$ & Polyvinyl Chloride \\
\hline PVES & Parity-Violating Electron Scattering \\
\hline PZT & Piezo-Electric Transducer \\
\hline QCD & Quantum Chromodynamics \\
\hline QDC & Charge-to-Digital Converter \\
\hline QE & Quantum Efficiency \\
\hline QED & Quantum Electrodynamics \\
\hline QTOR & Q-weak Toroidal Magnetic Spectrometer \\
\hline QTR & Q-weak Track Reconstruction Software \\
\hline QweakSimG4 & Q-weak Geant4 Monte Carlo Simulation Program \\
\hline $\mathrm{RCP}$ & Right-Handed Circularly Polarized \\
\hline RF & Radio Frequency \\
\hline
\end{tabular}




\begin{tabular}{|c|c|}
\hline RK4 & 4th-Order Runge-Kutta Method \\
\hline RMS & Root Mean Square \\
\hline ROC & Readout Controller \\
\hline RPV & R Parity-Violating \\
\hline $\mathrm{S} / \mathrm{B}$ & Signal-to-Background Ratio \\
\hline $\mathrm{S} / \mathrm{N}$ & Signal-to-Noise Ratio \\
\hline SEE & Switched Electrode Electronics \\
\hline SFR & Superconducting Radio Frequency \\
\hline SLAC & Stanford Linear Accelerator Center \\
\hline SLC & Stanford Linear Collider \\
\hline SM & Standard Model \\
\hline SUSY & Supersymmetry \\
\hline TDC & Time-to-Digital Converter \\
\hline TIR & Total Internal Reflection \\
\hline TRIUMF & Tri-University Meson Facility \\
\hline TS & Trigger Supervisor \\
\hline US & Upstream \\
\hline UV & Ultraviolet \\
\hline VDC & Vertical Drift Chamber \\
\hline VME & Versa Module Eurocard \\
\hline WCC & Weak Charged Current \\
\hline WNC & Weak Neutral Current \\
\hline
\end{tabular}




\section{Chapter 1}

\section{Theory and Motivation}

\subsection{Introduction}

The Standard Model (SM) of particle physics describes the interactions of elementary particles. This theory has successfully described a wide variety of phenomena in nuclear and particle physics and has been empirically tested through a large number of experiments over the past several decades. However, there are still reasons for us to believe that the SM is incomplete. Many questions cannot be answered by the SM, which suggests a more fundamental description of nature. Over years of efforts by physicists, many experiments have been carried out to test the SM and search for possible new physics. Direct searches for new particles are performed at high energy scale, such as those being performed at the Large Hadron Collider (LHC) at CERN (European Organization for Nuclear Research). Indirect searches are conducted at lower energies and seek to test the SM through precision measurements. Q-weak is an example of a low-energy experiment seeking to test the SM.

The greatest achievement of the SM lies in its success at incorporating quantum chromodynamics (QCD) and electroweak theory into a single framework based on a gauge field theory. Electroweak theory unifies the electromagnetic and weak interactions. The weak mixing angle $\theta_{W}$ characterizes the mixing between the electromagnetic (EM) and weak interactions. As with all coupling 
constants in quantum field theory, the weak mixing angle varies or "runs" with the momentum transfer of the interaction. Precision measurements of the running in turn allow a test of the SM. To date, the predicted running of the weak mixing angle has not been fully confirmed with existing data; more precise measurements of $\theta_{W}$ at low energy scale are needed.

The Q-weak collaboration [1] (see Appendix D for a collaboration list) at Jefferson Laboratory [2] is conducting a parity-violating electron scattering (PVES) experiment, to attempt an extraction of the weak mixing angle at low momentum transfer $\left(Q^{2}=0.026(\mathrm{GeV} / \mathrm{c})^{2}\right)$ through precise measurement of the proton's weak charge $Q_{W}^{p}$. The proton's weak charge will be determined by measuring the parity-violating asymmetry in elastic electron-proton scattering at low $Q^{2}$ and very forward angles. The goal of Q-weak is to determine the proton's weak charge to $4 \%$ combined systematic and statistical uncertainties, corresponding to $0.3 \%$ uncertainty in $\sin ^{2} \theta_{W}$ [3].

In this chapter, the physics of Q-weak will be reviewed, including the theoretical basis of the electroweak interaction, the methodology of PVES measurements, and the motivation to conduct such a difficult and precise experiment.

\subsection{Theoretical Background and Motivation}

\subsubsection{Electroweak Standard Model and the Weak Mixing Angle}

The Electroweak Standard Model unifies the electromagnetic interaction and the weak interaction based on the spontaneous symmetry breaking of the $S U(2)_{L} \times U(1)_{Y}$ local gauge group $[4,5,6,7]$. In this theory, elementary fermions (leptons and quarks) interact by exchanging vector gauge bosons. A fundamental parameter called the weak mixing angle (or Weinberg angle) $\theta_{W}$ characterizes the mixing of the $\mathrm{SU}(2)$ and $\mathrm{U}(1)$ spaces with gauge bosons $W_{\mu}^{i}, i=1,2,3$ and $B_{\mu}$. It describes the 
transformation of the original bosons as $[8,9]$ :

$$
\begin{aligned}
A & =B \cos \theta_{W}+W^{3} \sin \theta_{W}, & & \text { (massless photon field) } \\
W^{ \pm} & =\left(W^{1} \mp i W^{2}\right) / \sqrt{2}, & & \text { (massive charged weak boson field) } \\
Z & =-B \sin \theta_{W}+W^{3} \cos \theta_{W} . & & \text { (massive neutral weak boson field) }
\end{aligned}
$$

The resulting boson fields are a massless photon field $A$, two massive charged weak boson fields $W^{ \pm}$, and a massive neutral weak boson field $Z$. Masses are incorporated through spontaneous symmetry breaking via the Higgs mechanism [7, 10, 11]. The weak mixing angle relates the gauge coupling constants $g$ for $\mathrm{SU}(2)$ and $g^{\prime}$ for $\mathrm{U}(1)$ by:

$$
\sin \theta_{W}=\frac{e}{g}=\frac{g^{\prime}}{\sqrt{g^{2}+g^{\prime 2}}}, \quad \cos \theta_{W}=\frac{e}{g^{\prime}}=\frac{g}{\sqrt{g^{2}+g^{\prime 2}}}, \quad \tan \theta_{W}=\frac{g^{\prime}}{g}
$$

where $e$ represents the electric charge of a positron.

Fermions in this theory have three types of gauge interaction, with each Lagrangian density expressed in Einstein notation as [7]:

$$
\begin{aligned}
\mathcal{L}_{Q} & =-\frac{g g^{\prime}}{\sqrt{g^{2}+g^{\prime 2}}} \mathcal{J}_{E M}^{\mu} A_{\mu}, \\
\mathcal{L}_{Z} & =-\frac{\sqrt{g^{2}+g^{\prime 2}}}{2} \mathcal{J}_{Z}^{\mu} Z_{\mu}, \\
\mathcal{L}_{W} & =-\frac{g}{2 \sqrt{2}}\left(\mathcal{J}_{W}^{\mu} W_{\mu}^{-}+\mathcal{J}_{W}^{\mu \dagger} W_{\mu}^{+}\right),
\end{aligned}
$$

corresponding to the EM sector, weak charged sector and weak neutral sector, respectively (see the Feynman diagrams in Fig. 1.1). $\mathcal{J}^{\mu}$ are fermion currents; $A_{\mu}, Z_{\mu}$ and $W_{\mu}^{ \pm}$represent boson fields; the upper index $\mu$ indicates a contravariant component and the lower index $\mu$ indicates a covariant component. The factors involving couplings of $g$ and $g^{\prime}$ in the Lagrangian expressions may also be represented by the weak mixing angle according to Eqn. 1.4. 

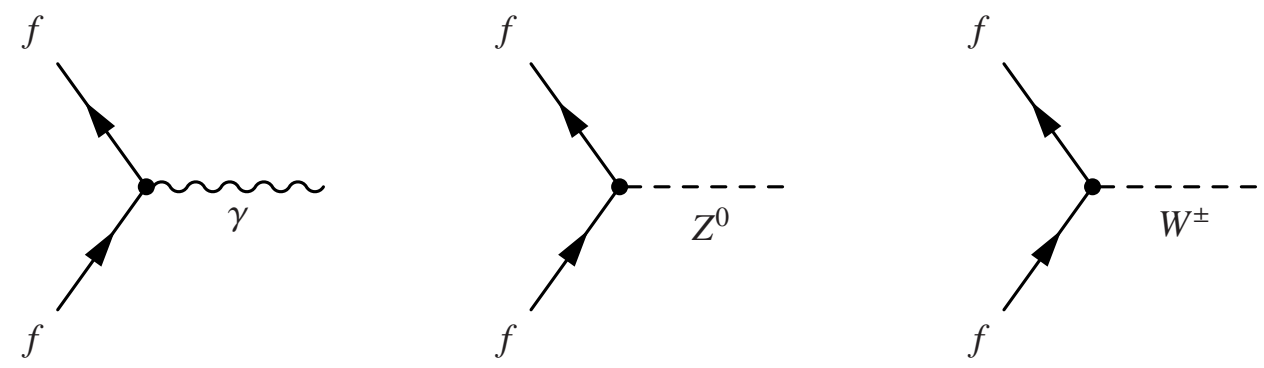

Figure 1.1: Fermion gauge interactions in the standard electroweak model.

\section{The QED Interaction}

Eqn. 1.5 describes the EM interaction [7] between a massless photon field $A_{\mu}$ and the fermion EM current

$$
\mathcal{J}_{E M}^{\mu}=\sum_{i} q_{i} \bar{\psi}_{i} \gamma^{\mu} \psi_{i}
$$

where the sum is over all fermion currents running over all quarks and leptons, $q_{i}$ is the electric charge of the fermion in units of $e, \gamma^{\mu}(\mu=0,1,2,3)$ are Dirac matrices, $\bar{\psi}_{i}$ and $\psi_{i}$ are the annihilation operator and creation operator, respectively.

\section{The Weak Neutral Current Interaction}

Eqn. 1.6 represents weak neutral current interaction $[12,7,13]$ between a massive $Z^{0}$ boson field and the fermion weak neutral current (WNC):

$$
\mathcal{J}_{Z}^{\mu}=\sum_{i} \bar{\psi}_{i} \gamma^{\mu}\left[g_{V}^{i}+g_{A}^{i} \gamma^{5}\right] \psi_{i}
$$

where $\gamma_{5}=i \gamma^{0} \gamma^{1} \gamma^{2} \gamma^{3}, g_{V}^{i}$ and $g_{A}^{i}$ are the vector coupling and axial-vector coupling of the fermion. They are given by $[7,9,13]$ :

$$
\begin{aligned}
& g_{V}^{i}=2 T_{3}^{i}-4 q_{i} \sin ^{2} \theta_{W}, \\
& g_{A}^{i}=-2 T_{3}^{i},
\end{aligned}
$$


in which $T_{3}^{i}$ is the 3 rd component of the weak isospin of fermion $i$ and has the value of $+\frac{1}{2}$ or $-\frac{1}{2}, q_{i}$ is the electric charge of the fermion in units of $e$. The couplings $g_{V}^{i}$ and $g_{A}^{i}$ reflect the weak vector charge and weak axial charge of fermions. In the SM at tree level, the lowest order in perturbation theory, the values of weak vector charge, weak axial charge, along with electric charge for the elementary fermions are listed in Table 1.1.

\begin{tabular}{c|crcc}
\hline \hline & Fermion & $q$ & $g_{V}$ & $g_{A}$ \\
\hline Leptons & $v_{e}, v_{\mu}, v_{\tau}$ & 0 & 1 & -1 \\
& $e, \mu, \tau$ & -1 & $-1+4 \sin ^{2} \theta_{W}$ & 1 \\
Quarks & $u, c, t$ & $\frac{2}{3}$ & $1-\frac{8}{3} \sin ^{2} \theta_{W}$ & -1 \\
& $d, s, b$ & $-\frac{1}{3}$ & $-1+\frac{4}{3} \sin ^{2} \theta_{W}$ & 1 \\
\hline \hline
\end{tabular}

Table 1.1: Electric and weak charges of elementary fermions in the Standard Model at tree level [14].

For a description of the parity-violating electron scattering process, a set of SM parameters for quark weak couplings, $C_{1 f}$ and $C_{2 f}\left(f=u, c, t, d, s, b\right.$ quarks), is also commonly used. The $C_{1 f}$ and $C_{2 f}$ are low energy effective PV couplings, characterizing the WNC interaction between an electron and a quark. This set of quark parameters is related to the above parameters $g_{V}$ and $g_{A}$ by:

$$
\begin{aligned}
g_{V}^{f} & =-2 C_{1 f}, \\
g_{A}^{f} & =\frac{2 C_{2 f}}{1-4 \sin ^{2} \theta_{W}} .
\end{aligned}
$$

The $C_{1 f}$ and $C_{2 f}$ correspond to the weak vector charge and axial charge of quarks, respectively. Explicit expressions for these quark weak couplings are summarized in Table 1.2. The Q-weak measurement is expected to establish constraints on $C_{1 u}$ and $C_{1 d}$, which will be discussed in Section 1.4.1. 


\begin{tabular}{c|cc}
\hline \hline Quarks & $C_{1 f}$ & $C_{2 f}$ \\
\hline$u, c, t$ & $-\frac{1}{2}\left(1-\frac{8}{3} \sin ^{2} \theta_{W}\right)$ & $-\frac{1}{2}\left(1-4 \sin ^{2} \theta_{W}\right)$ \\
$d, s, b$ & $-\frac{1}{2}\left(-1+\frac{4}{3} \sin ^{2} \theta_{W}\right)$ & $\frac{1}{2}\left(1-4 \sin ^{2} \theta_{W}\right)$ \\
\hline \hline
\end{tabular}

Table 1.2: Quark weak couplings at tree level [9].

\section{The Weak Charged Current Interaction}

Eqn. 1.7 describes the weak charged current (WCC) interaction $[12,7]$ in which a massive $W^{ \pm}$ boson field is coupled to a charged current

$$
\mathcal{J}_{W}^{\mu}=\sum_{r} \bar{\psi}_{i}^{\prime} \gamma^{\mu}\left(1-\gamma^{5}\right) \psi_{i}
$$

Being different from the electromagnetic and neutral currents, in which only fields with the same charge can be mixed, the weak charged current requires one charge to be raised or lowered; therefore, the $\bar{\psi}_{i}^{\prime}$ and $\psi_{i}$ in Eqn. 1.14 represent fermion fields from different particles. Since only QED and WNC interactions are involved in the parity-violating elastic electron scattering processes, we end the discussion of the WCC interaction here.

\subsubsection{Running of the Weak Mixing Angle}

Radiative corrections in the SM (also discussed later in section 1.3.5) are responsible for the variation of the effective QED coupling $\alpha\left(\mu^{2}\right)$ and QCD coupling $\alpha_{s}\left(\mu^{2}\right)$ with energy scale $\mu$. This variation is referred to as "running" with energy scale. The running has been well established and can be predicted to high precision, and is regarded as one of the triumphs of Quantum Field The-

ory. Analogous to $\alpha\left(\mu^{2}\right)$ and $\alpha_{s}\left(\mu^{2}\right)$, the effective weak mixing angle $\theta_{W}\left(\mu^{2}\right)$ also runs. This is a key prediction made by the electroweak SM $[15,16]$. Fig. 1.2 displays the running of $\sin ^{2} \theta_{W}$ with momentum transfer $Q(\mu \sim Q)$ in the $\overline{\mathrm{MS}}$ (modified minimal subtraction) scheme [9, 15]. The width of the blue line reflects the theoretical uncertainty. The precise measurements in electron-positron 
colliding beam experiments at the $Z$-pole pin the overall magnitude of the curve. The minimum of the curve corresponds to the electroweak scale $Q=M_{W}$.

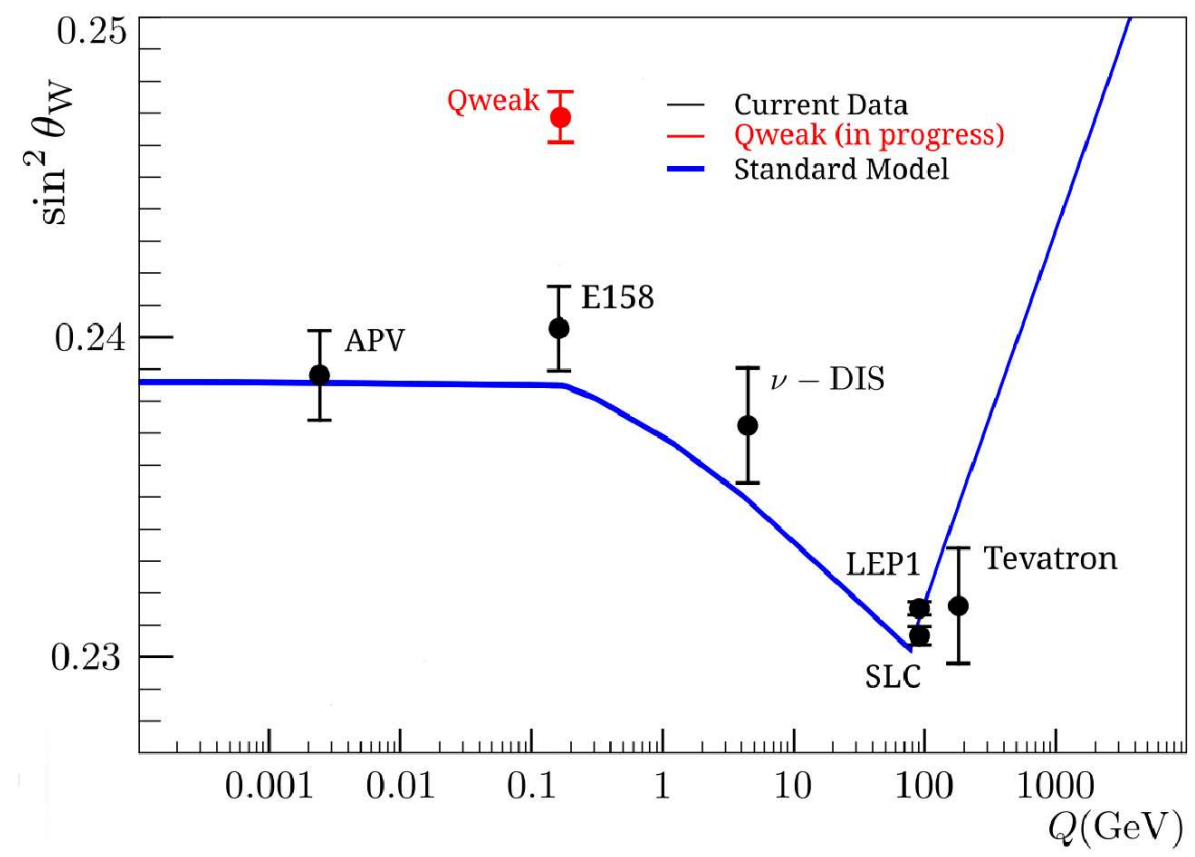

Figure 1.2: Running of the weak mixing angle. The solid blue line shows the calculated running of $\sin ^{2} \theta_{W}$ in the Standard Model, defined in the $\overline{\mathrm{MS}}$ scheme. The low energy measurements at various scales are from atomic parity violation (APV) [17, 18], deep inelastic neutrino-nucleus scattering (NuTeV) [19], elastic Møller scattering (SLAC E158) [20] and Z-pole asymmetries (LEP+SLC) [21, 22]. The Q-weak e-p elastic scattering measurements are in progress. The data point has been displaced from the theoretical curve to make the size of the error bar more apparent.

By comparing this prediction with precise experimental results, the electroweak theory of the SM can be tested. A deviation would indicate new physics beyond the SM. To test the running, a set of precision measurements at different $Q$ is required. To date, unlike the gauge couplings of QED and QCD, whose running below the weak scale has been stringently tested in a variety of ways, the low-energy running of $\sin ^{2} \theta_{W}$ has not been precisely confirmed.

The most accurate measurements have been performed at LEP (the CERN $e^{+} e^{-}$collider) and SLC (the Stanford Linear Collider) [21, 22] near the $Z^{0}$ pole, which fix the value of $\sin ^{2} \theta_{W}$ precisely near the electroweak scale. An atomic parity violation (APV) experiment [17, 18] extracted the 
weak charge of the cesium nucleus $\left(Q_{W}(C s)\right)$, hence the value of $\sin ^{2} \theta_{W}$ at very low $Q^{2}$. The $\mathrm{NuTeV}$ experiment [19] at Fermilab extracted the value of $\sin ^{2} \theta_{W}$ at $Q \sim 3 \mathrm{GeV}$ through deep inelastic scattering of neutrinos from a $\mathrm{BeO}$ target. The $\mathrm{NuTeV}$ result exhibits a large deviation from the SM prediction, but it is still a subject of debate, as the source of deviation is not clearly known yet [23]. The E158 experiment at SLAC [20] was a parity-violating Møller scattering experiment performed at low $Q^{2} \sim 0.026 \mathrm{GeV}^{2}$. The reported result is consistent with the SM expectation. The Q-weak experiment at JLab is performing a parity-violating e-p elastic scattering measurement near the same $Q^{2}$ as SLAC E158. The JLab $12 \mathrm{GeV}$ Møller and e-DIS measurements have also been proposed for future data points [24].

\subsection{Methodology of Measuring $Q_{W}^{p}$}

To lowest order in perturbation theory (tree level), the proton's weak charge $\left(Q_{W}^{p}\right.$, the vector coupling) is expressed as

$$
Q_{W}^{p}=1-4 \sin ^{2} \theta_{W}
$$

Since the numerical value of $\sin ^{2} \theta_{W}$ is approximately $1 / 4$, any small change of $\sin ^{2} \theta_{W}$ due to new physics would be relatively enhanced in the observable $Q_{W}^{p}$. Conversely, $\sin ^{2} \theta_{W}$ can be extracted to high precision with a relatively lower precision extraction of $Q_{W}^{p}$. The Q-weak experiment uses parity-violating elastic electron-proton (e-p) scattering as a tool to measure the proton's weak charge via the weak neutral current.

\subsection{1 e-N Elastic Scattering Kinematics}

For the process of an electron $e^{-}$elastically scattering from a nucleon $N$ (proton or neutron),

$$
e^{-}\left(P_{1}\right)+N\left(P_{2}\right) \longrightarrow e^{-}\left(P_{3}\right)+N\left(P_{4}\right)
$$


with the four-momenta of the initial $e^{-}$and $N$ defined as $P_{1}$ and $P_{2}$, and the four-momenta of final $e^{-}$and $N$ as $P_{3}$ and $P_{4}$, respectively. The four-momentum for each particle is commonly expressed in terms of its energy $E$ and three-momentum $\vec{p}$, denoted by a contravariant and a covariant fourvector:

$$
P^{\mu}=(E, \vec{p}), \quad P_{\mu}=g_{\mu \nu} P^{v}=(E,-\vec{p}),
$$

where $\mu, v=0,1,2,3$ (i.e. $P^{0}=E, P^{1}=p_{x}, P^{2}=p_{y}, P^{3}=p_{z}$ ), $g_{\mu \nu}$ are the elements of the Minkowski tensor

$$
g_{\mu \nu}=g^{\mu \nu}=\left(\begin{array}{cccc}
1 & 0 & 0 & 0 \\
0 & -1 & 0 & 0 \\
0 & 0 & -1 & 0 \\
0 & 0 & 0 & -1
\end{array}\right) \text {. }
$$

In Einstein notation, for a particle of mass $m$, the four-momentum squared is given by:

$$
P^{2}=P^{\mu} P_{\mu}=E^{2}-|\vec{p}|^{2}=m^{2}
$$

Units with $\hbar=c=1$ are employed in this discussion.

The four-momentum transferred from the electron to the nucleon can be obtained from $q=$ $P_{1}-P_{3}$. We define the Lorentz invariant $Q^{2}$ as:

$$
Q^{2}=-q^{2}=-\left(P_{1}-P_{3}\right)^{2} \simeq 4 E_{1} E_{3} \sin ^{2} \frac{\theta}{2},
$$

where $E_{1}$ and $E_{3}$ are the energies of the incident and scattered electrons, and $\theta$ is the electron's scattering angle, in the lab frame. Assuming elastic scattering, the value of $E_{3}$ can be related to $E_{1}$ and $\theta$ by

$$
E_{3}=\frac{E_{1}}{1+\frac{E_{1}}{M}(1-\cos \theta)},
$$

where $M$ is the rest mass of the nucleon. 


\subsubsection{Scattering Amplitude and Cross-section}

The process of an electron elastically scattering from a nucleon involves both the EM interaction and the WNC interaction. The interference between the two amplitudes leads to parity violation. To leading order in electroweak theory, the electron-nucleon (e-N) elastic scattering process is described by the Feynman diagrams in Figure 1.3, representing single $\gamma$ exchange for the EM interaction and $Z^{0}$ exchange for the WNC interaction.
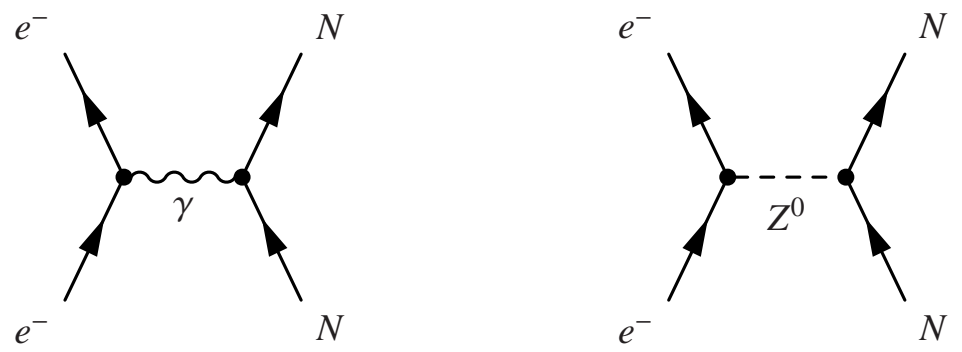

Figure 1.3: EM (left) and WNC (right) amplitudes for elastic e-N scattering.

The interaction amplitude therefore contains both electromagnetic and weak neutral current contributions, and the total invariant amplitude is a coherent sum of $\mathcal{M}_{\gamma}$ and $\mathcal{M}_{Z}$ :

$$
\mathcal{M}=\mathcal{M}_{\gamma}+\mathcal{M}_{Z}
$$

\section{Amplitude for $\gamma$ exchange}

The amplitude for the EM interaction is [25]

$$
\mathcal{M}_{\gamma}=4 \pi \alpha \mathcal{T}_{E M}^{e, \mu} \frac{g_{\mu v}}{Q^{2}} \mathcal{J}_{E M}^{N, v}
$$

where $Q^{2}$ is the four-momentum transfer squared, $g_{\mu \nu}$ are the elements of the Minkowski tensor, and $\alpha$ is the fine structure constant. $\mathcal{J}_{E M}^{e, \mu}$ and $\mathcal{J}_{E M}^{N, v}$ represent the matrix elements of the EM currents 
for a point-like electron and structured nucleon, respectively. They can be expressed as [25, 10]

$$
\mathcal{J}_{E M}^{e, \mu}=Q^{\prime} \overline{\mathcal{U}}_{e}\left(P_{3}, s_{3}\right) \gamma^{\mu} \mathcal{U}_{e}\left(P_{1}, s_{1}\right)
$$

and

$$
\begin{aligned}
\mathcal{J}_{E M}^{N, \mu} & =\overline{\mathcal{U}}_{N}\left(P_{4}, s_{4}\right) \Gamma_{\gamma}^{\mu} \mathcal{U}_{N}\left(P_{2}, s_{2}\right) \\
& =\overline{\mathcal{U}}_{N}\left(P_{4}, s_{4}\right)\left[\gamma^{\mu} F_{1}^{\gamma}\left(Q^{2}\right)+\frac{i \sigma^{\mu v} q_{v}}{2 M} F_{2}^{\gamma}\left(Q^{2}\right)\right] \mathcal{U}_{N}\left(P_{2}, s_{2}\right)
\end{aligned}
$$

The $\mathcal{U}$ in the above equations is a Dirac spinor, which is a 4-component vector, depending on momentum $P$ and spin state $s$ ( $s=1,2$ for spin "up" or "down"). Defining the helicity operator $\hat{h}=\vec{\sigma} \cdot \vec{p} /|\vec{p}|$ with the Pauli spin matrices $\vec{\sigma}$, the explicit form for the Dirac spinor in the Pauli-Dirac representation can be written as $[7,10]$ :

$$
\mathcal{U}(P, s)=\sqrt{E_{\vec{p}}+m}\left(\begin{array}{c}
\chi_{s} \\
\frac{\vec{\sigma} \cdot \vec{p}}{E_{\vec{p}}+m} \chi_{s}
\end{array}\right),
$$

where

$$
\chi_{1}=\left(\begin{array}{l}
1 \\
0
\end{array}\right) \quad \text { and } \quad \chi_{2}=\left(\begin{array}{c}
0 \\
1
\end{array}\right)
$$

represent two spin states of the particle: parallel and anti-parallel to its momentum direction, and $E_{\vec{p}}=+\sqrt{m^{2}+\vec{p}^{2}} \cdot \overline{\mathcal{U}} \equiv \mathcal{U}^{\dagger} \gamma^{0}$ is the Dirac adjoint. In Eqn. 1.24, $Q^{\prime}$ represents the electromagnetic charge. In Eqn. 1.25, $F_{1}^{\gamma}$ and $F_{2}^{\gamma}$ are the EM Dirac and Pauli form factors that represent the internal structure of the nucleon, $M$ is the nucleon mass, and $\sigma^{\mu v} \equiv \frac{i}{2}\left[\gamma^{\mu}, \gamma^{v}\right]$. 


\section{Amplitude for $Z$ boson exchange}

The amplitude for the WNC interaction is $[25,10]$

$$
\mathcal{M}_{Z}=\frac{g^{2}}{16 \cos ^{2} \theta_{W}} \mathcal{J}_{Z}^{e, \mu} \frac{g_{\mu \nu}-q_{\mu} q_{v} / M_{Z}^{2}}{Q^{2}-M_{Z}^{2}} \mathcal{J}_{Z}^{N, v},
$$

where $M_{Z}$ is the mass of $Z^{0}$ boson, $\mathcal{J}_{Z}^{e, \mu}$ and $\mathcal{J}_{Z}^{N, v}$ are neutral currents for electron and nucleon, respectively:

$$
\mathcal{J}_{Z}^{e, \mu}=\overline{\mathcal{U}}_{e}\left(P_{3}, s_{3}\right) \gamma^{\mu}\left(g_{V}^{e}+g_{A}^{e} \gamma^{5}\right) \mathcal{U}_{e}\left(P_{1}, s_{1}\right)=\mathcal{J}_{Z, V}^{e, \mu}+\mathcal{J}_{Z, A}^{e, \mu}
$$

and

$$
\begin{aligned}
\mathcal{J}_{Z}^{N, \mu} & =\overline{\mathcal{U}}_{N}\left(P_{4}, s_{4}\right) \Gamma_{Z}^{\mu} \mathcal{U}_{N}\left(P_{2}, s_{2}\right) \\
& =\overline{\mathcal{U}}_{N}\left(P_{4}, s_{4}\right)\left[\gamma^{\mu} F_{1}^{Z}\left(Q^{2}\right)+\frac{i \sigma^{\mu v} q_{v}}{2 M} F_{2}^{Z}\left(Q^{2}\right)+\gamma^{\mu} \gamma_{5} G_{A}^{Z}\left(Q^{2}\right)\right] \mathcal{U}_{N}\left(P_{2}, s_{2}\right) \\
& =\mathcal{J}_{Z, V}^{N, \mu}+\mathcal{J}_{Z, A}^{N, \mu},
\end{aligned}
$$

where $F_{1}^{Z}$ and $F_{2}^{Z}$ are weak (vector) Dirac and Pauli form factors, and $G_{A}^{Z}$ is the axial form factor. The Sachs electric and magnetic form factors [26] are defined as linear combinations of the Dirac and Pauli form factors:

$$
\begin{aligned}
& G_{E}^{\gamma(Z)}\left(Q^{2}\right)=F_{1}^{\gamma(Z)}\left(Q^{2}\right)-\tau F_{2}^{\gamma(Z)}\left(Q^{2}\right), \\
& G_{M}^{\gamma(Z)}\left(Q^{2}\right)=F_{1}^{\gamma(Z)}\left(Q^{2}\right)+F_{2}^{\gamma(Z)}\left(Q^{2}\right),
\end{aligned}
$$

where $\tau=\frac{Q^{2}}{4 M^{2}}$ is a kinematic factor. In the Breit frame [27] of the elastic electron scattering system, these form factors correspond to the Fourier transforms of the charge and magnetization densities of the nucleon. In the $Q^{2} \rightarrow 0$ limit, $G_{E}^{\gamma}$ is equal to the normalized electric charge, and $G_{M}^{\gamma}$ coincides with the magnetic moment. In terms of the Sachs form factors, the EM current and weak neutral 
current for the nucleon in Eqn. 1.25 and Eqn. 1.30 can be expressed as:

$$
\begin{gathered}
\mathcal{J}_{E M}^{N, \mu}=\overline{\mathcal{U}}_{N}\left(P_{4}, s_{4}\right)\left[G_{M}^{\gamma, N} \gamma^{\mu}-\left(P_{4}^{\mu}+P_{2}^{\mu}\right) \frac{1}{2 M_{N}} \frac{G_{M}^{\gamma, N}-G_{E}^{\gamma, N}}{1+\tau}\right] \mathcal{U}_{N}\left(P_{2}, s_{2}\right), \\
\mathcal{J}_{Z}^{N, \mu}=\overline{\mathcal{U}}_{N}\left(P_{4}, s_{4}\right)\left[G_{M}^{Z, N} \gamma^{\mu}-\left(P_{4}^{\mu}+P_{2}^{\mu}\right) \frac{1}{2 M_{N}} \frac{G_{M}^{Z, N}-G_{E}^{Z, N}}{1+\tau}+\gamma^{\mu} \gamma_{5} G_{A}^{Z, N}\right] \mathcal{U}_{N}\left(P_{2}, s_{2}\right) .
\end{gathered}
$$

\section{Elastic Scattering Cross-section}

In the Born approximation, the differential cross-section can be written as [28]:

$$
\frac{d \sigma}{d \Omega}=\left(\frac{\alpha}{4 M Q^{2}} \frac{E^{\prime}}{E_{0}}\right)^{2}|\mathcal{M}|^{2}
$$

where $E^{\prime}$ and $E_{0}$ are the final and initial energies of the scattered electrons. From Eqn. 1.22, the squared amplitude includes the following three terms:

$$
\begin{aligned}
|\mathcal{M}|^{2} & =\left|\mathcal{M}_{\gamma}+\mathcal{M}_{Z}\right|^{2}=\left(\mathcal{M}_{\gamma}+\mathcal{M}_{Z}\right)\left(\mathcal{M}_{\gamma}+\mathcal{M}_{Z}\right)^{*} \\
& =\left|\mathcal{M}_{\gamma}\right|^{2}+2 \Re\left(\mathcal{M}_{\gamma}^{*} \mathcal{M}_{Z}\right)+\left|\mathcal{M}_{Z}\right|^{2}
\end{aligned}
$$

The purely weak parity-conserving (PC) term $\left|\mathcal{M}_{Z}\right|^{2}$ and the parity-violating (PV) interference term $2 \Re\left(\mathcal{M}_{\gamma}^{*} \mathcal{M}_{Z}\right)$ are small, compared to the purely EM parity-conserving term $\left|\mathcal{M}_{\gamma}\right|^{2}$. Therefore, $|\mathcal{M}|^{2} \simeq$ $\left|\mathcal{M}_{\gamma}\right|^{2}$, and the $\left|\mathcal{M}_{\gamma}\right|^{2}$ term provides the dominant contribution to the cross-session, which is well known according to the Rosenbluth Formula [29]:

$$
\frac{d \sigma}{d \Omega}=\left(\frac{d \sigma}{d \Omega}\right)_{M o t t}\left[\left(\frac{G_{E}^{\gamma 2}+\tau G_{M}^{\gamma 2}}{1+\tau}\right)+2 \tau G_{M}^{\gamma 2} \tan ^{2} \frac{\theta}{2}\right] .
$$

The Sachs form factors $G_{E}^{\gamma}$ and $G_{M}^{\gamma}$ are functions of $Q^{2}$ and have been determined by other experiments [30]. Here, the Mott cross-section describing the scattering from a point-like spin-0 target is 
given by [8]:

$$
\left.\frac{d \sigma}{d \Omega}\right|_{\text {Mott }}=\frac{(\hbar c \alpha)^{2}}{4 E_{0}^{2} \sin ^{4}(\theta / 2)}\left(\frac{E^{\prime}}{E_{0}}\right) \cos ^{2}(\theta / 2)=\frac{(\hbar c \alpha)^{2} \cos ^{2}(\theta / 2)}{4 E_{0}^{2} \sin ^{4}(\theta / 2)\left[1+2 \frac{E_{0}}{M} \sin ^{2}(\theta / 2)\right]}
$$

\subsubsection{Parity-Violating Asymmetry}

For longitudinally polarized electrons scattering elastically from an unpolarized nucleon, the relative difference in the scattering cross section $d \sigma$ between the two helicity states of the electron is defined as the parity-violating asymmetry [9]

$$
A_{P V}=\frac{d \sigma_{R}-d \sigma_{L}}{d \sigma_{R}+d \sigma_{L}}
$$

where the subscripts " $L$ " and " $R$ " refer to the helicity states of the left- and right-handed polarized electrons. In terms of the interaction amplitudes, this is equivalent to [28]:

$$
A_{P V}=\frac{\left|\mathcal{M}^{R}\right|^{2}-\left|\mathcal{M}^{L}\right|^{2}}{\left|\mathcal{M}^{R}\right|^{2}+\left|\mathcal{M}^{L}\right|^{2}}
$$

where $\mathcal{M}^{R}$ and $\mathcal{M}^{L}$ are the amplitudes for the right-handed and left-handed helicity states, respectively, and

$$
\mathcal{M}^{R, L}=\mathcal{M}_{\gamma}+\mathcal{M}_{Z}^{R, L}
$$

The $\gamma$-exchange amplitudes are the same for left- and right-handed electrons since EM interactions conserve parity, while the Z-exchange amplitudes are different as they involve parity-violating processes. The $\mathcal{M}_{Z}^{R}$ and $\mathcal{M}_{Z}^{L}$ are calculated by applying chiral projection operators to the Dirac spinor of the electron [31]:

$$
\mathcal{U}_{e}(P, s) \longmapsto\left(\frac{1 \pm \gamma^{5}}{2}\right) \mathcal{U}_{e}(P, s)
$$

By replacing the $\mathcal{U}_{e}(P, s)$ by $\frac{1}{2}\left(1 \pm \gamma^{5}\right) \mathcal{U}_{e}(P, s)$ in Eqn. 1.29 for the positive-helicity $(R)$ and negativehelicity $(L)$ electrons, the matrix elements for the neutral weak interaction between a polarized 
electron and an unpolarized nucleon can be explicitly written as:

$$
\begin{aligned}
\mathcal{M}_{Z}^{R}= & \sin ^{2} \theta_{W} \overline{\mathcal{U}}_{e}\left(P_{3}, s_{3}\right) \gamma_{\mu}\left(\frac{1+\gamma^{5}}{2}\right) \mathcal{U}_{e}\left(P_{1}, s_{1}\right)\left(\frac{-1}{Q^{2}-M_{Z}^{2}}\right) \\
& \overline{\mathcal{U}}_{N}\left(P_{4}, s_{4}\right)\left[G_{M}^{Z, N} \gamma^{\mu}-\left(P_{4}^{\mu}+P_{2}^{\mu}\right) \frac{1}{2 M_{N}} \frac{G_{M}^{Z, N}-G_{E}^{Z, N}}{1+\tau}+\gamma^{\mu} \gamma_{5} G_{A}^{Z, N}\right] \mathcal{U}_{N}\left(P_{2}, s_{2}\right)
\end{aligned}
$$

and

$$
\begin{aligned}
\mathcal{M}_{Z}^{L}= & \left(-\frac{1}{2}+\sin ^{2} \theta_{W}\right) \overline{\mathcal{U}}_{e}\left(P_{3}, s_{3}\right) \gamma_{\mu}\left(\frac{1-\gamma^{5}}{2}\right) \mathcal{U}_{e}\left(P_{1}, s_{1}\right)\left(\frac{-1}{Q^{2}-M_{Z}^{2}}\right) \\
& \overline{\mathcal{U}}_{N}\left(P_{4}, s_{4}\right)\left[G_{M}^{Z, N} \gamma^{\mu}-\left(P_{4}^{\mu}+P_{2}^{\mu}\right) \frac{1}{2 M_{N}} \frac{G_{M}^{Z, N}-G_{E}^{Z, N}}{1+\tau}+\gamma^{\mu} \gamma_{5} G_{A}^{Z, N}\right] \mathcal{U}_{N}\left(P_{2}, s_{2}\right) .
\end{aligned}
$$

In Eqn. 1.40, the parity-conserving contributions cancel in the numerator of $A_{P V}$, so that the asymmetry is sensitive primarily to the $\gamma$-Z interference term. The scale of the PV asymmetry is typically the $10^{-6}$ (ppm level), which is determined by the relative strength of the EM and weak interactions at typical $Q^{2} \sim \mathrm{GeV}^{2}$.

Using the above expressions, $A_{P V}$ may be obtained in terms of the various Sachs form factors as $[31,28]$ :

$$
A_{P V}=-\frac{G_{F} Q^{2}}{4 \sqrt{2} \pi \alpha}\left[\frac{\varepsilon G_{E}^{\gamma} G_{E}^{Z}+\tau G_{M}^{\gamma} G_{M}^{Z}-\left(1-4 \sin ^{2} \theta_{W}\right) \varepsilon^{\prime} G_{M}^{\gamma} G_{A}^{e}}{\varepsilon\left(G_{E}^{\gamma}\right)^{2}+\tau\left(G_{M}^{\gamma}\right)^{2}}\right],
$$

where

$$
G_{F}=\frac{\pi \alpha}{\sqrt{2} M_{Z}^{2} \sin ^{2} \theta_{W} \cos ^{2} \theta_{W}}=1.16639 \times 10^{5} \quad \mathrm{GeV}^{-2}
$$

is the Fermi constant [9] which governs the strength of the weak interaction. The kinematic factors $\varepsilon$ and $\varepsilon^{\prime}$ are:

$$
\varepsilon=\left[1+2(1+\tau) \tan ^{2} \frac{\theta}{2}\right]^{-1}, \quad \text { and } \quad \varepsilon^{\prime}=\left[\tau(1+\tau)\left(1-\varepsilon^{2}\right)\right]^{1 / 2}
$$

The Sachs form factors indicate the substructure of the proton target. In the $Q^{2} \rightarrow 0$ limit, the weak neutral form factor $G_{E}^{Z}\left(Q^{2}\right)$ coincides with the proton's weak charge $Q_{W}^{p}$. The neutral 
weak interaction also involves an axial form factor $G_{A}^{e}$ in the third term of the numerator. In this expression, the notation for the axial form factor is modified to $G_{A}^{e}$ from the previously used $G_{A}^{Z}$. The difference between $G_{A}^{Z}$ and $G_{A}^{e}$ lies in radiative corrections. $G_{A}^{Z}$ involves leading order $Z$-exchange contribution, while $G_{A}^{e}$ includes more contributions from anapole effects and other electroweak radiative corrections [32]. The relationship between them can be expressed as:

$$
G_{A}^{e}=G_{A}^{Z}+\eta F_{A}+R_{e}
$$

where $\eta=\frac{8 \pi \sqrt{2} \alpha}{1-4 \sin ^{2} \theta_{W}}, F_{A}$ represents the nucleon anapole form factor, and $R_{e}$ are radiative corrections. Typical contributions to $F_{A}$ and $R_{e}$ are shown in Fig. 1.6. The anapole type effects associated with the " $\gamma Z$ mixing" amplitudes are the dominant correction [33]. Therefore, $G_{A}^{Z}$ is traditionally replaced by $G_{A}^{e}$ here to reflect the higher order diagrams involving electromagnetic interactions.

\subsubsection{Extracting $Q_{w}^{p}$}

The Q-weak experiment is performing an elastic e-p scattering asymmetry measurement at small $Q^{2}$ and forward angles. In the limit $Q^{2} \rightarrow 0$ and $\theta \rightarrow 0$, the kinematic factors have the limiting behavior $\epsilon \rightarrow 1$ and $\tau \ll 1$. In this limit, the asymmetry can be expressed as [15, 16]:

$$
A_{P V}=\frac{-G_{F}}{4 \pi \alpha \sqrt{2}}\left[Q^{2} Q_{W}^{p}+F^{p}\left(Q^{2}, \theta\right)\right] \underset{Q^{2} \rightarrow 0}{\stackrel{\theta \rightarrow 0}{\longrightarrow}} \frac{-G_{F}}{4 \pi \alpha \sqrt{2}}\left[Q^{2} Q_{W}^{p}+Q^{4} B\left(Q^{2}\right)\right]
$$

where $F^{p}\left(Q^{2}, \theta\right)$ and $B\left(Q^{2}\right)$ represent hadronic form factors, and $Q_{W}^{p}$ is the proton's weak charge.

The asymmetry $A_{P V}$ under this condition includes one $Q_{W}^{p}$ term for a point-like proton and one hadronic structure term, providing corrections. At Q-weak kinematics, the asymmetry is approximately proportional to the leading term $Q^{2} Q_{W}^{p}$, and $Q_{W}^{p}=1-4 \sin ^{2} \theta_{W}$ at tree level.

The nucleon structure contributions contained in $B\left(Q^{2}\right)$ can be reduced by measuring $A_{P V}$ at low momentum transfer, since $B\left(Q^{2}\right)$ enters to order $Q^{4}$, while the $Q_{W}^{p}$ enters to order $Q^{2}$. However, a side-effect of lowering the momentum transfer is that it also reduces the value of $A_{P V}$ and hence the sensitivity to $Q_{W}^{p}$. The Q-weak experiment design reached a compromise between these two effects 
by selecting an average $Q^{2}=0.026(\mathrm{GeV} / \mathrm{c})^{2}$; at this value, the hadronic term contributes $\sim 1 / 3$ to the total asymmetry of $\sim 230 \mathrm{ppb}[34]$.

The proton's weak charge and the hadronic form factor $B\left(Q^{2}\right)$, containing the contributions from the nucleon electric, magnetic and weak (or equivalently strange) form factors, can be extrapolated via empirical fits from previous experiments, such as SAMPLE at MIT-Bates, G0 and HAPPEx at JLab and PVA4 at Mainz (see [35, 36, 37, 38, 39, 40] and references within). Thus, once the parity-violating asymmetry $A_{P V}$ is precisely measured by Q-weak, and accurate knowledge of the hadronic form factors is obtained, the proton's weak charge and hence the weak mixing angle can be determined.

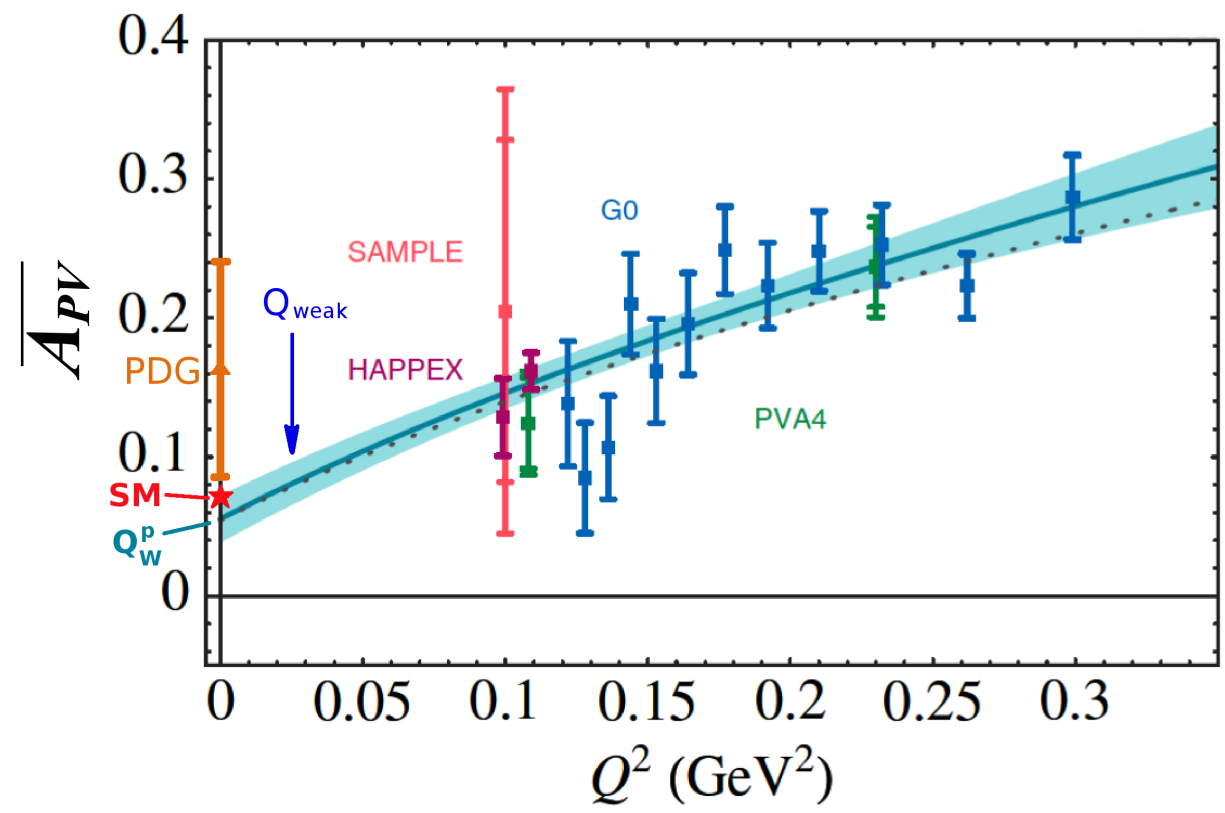

Figure 1.4: Global fit of normalized PVES asymmetries $\overline{A_{P V}}$ (defined by Eqn. 1.50) and $Q_{W}^{p}$ extrapolation to $Q^{2}=0$ [41]. The triangular data point indicates the previous data, summarized by the Particle Data Group (PDG) [9]; the star indicates the Standard Model prediction [15]; the solid curve and shaded region indicate the best fit of existing data and $1 \sigma$ bound, respectively. The dashed line is the fit including theoretical estimates of the anapole form factors [42].

Fig. 1.4 shows such an empirical extrapolation [40] with the existing world data from a number of PVES experiments, measuring parity violation over the $Q^{2}$ range of $0.1-0.3 \mathrm{GeV}^{2}$. In this 
analysis, data were fitted with a systematic expansion of the relevant hadronic form factors in powers of $Q^{2}$; the parity-violating asymmetries were normalized as:

$$
\overline{A_{P V}}\left(Q^{2}\right)=\frac{A_{P V}}{\frac{-G_{F}}{4 \pi \alpha \sqrt{2}} Q^{2}}=Q_{W}^{p}+Q^{2} B\left(Q^{2}\right),
$$

which is a dimensionless, $Q^{2}$-dependent function, referred to as the normalized PV asymmetry. Extrapolation of $\overline{A_{P V}}$ for world data to the forward-angle limit $\theta \rightarrow 0$ yields the value of the proton's weak charge $Q_{W}^{p}$. The extrapolation gives the global fit value of $Q_{W(\text { Fit })}^{p}\left(Q^{2}=0\right)=0.055 \pm 0.017$, which has a relative uncertainty of $31 \%$. A prediction of the proton's weak charge made in the Standard Model (see discussion in the next section) gives a theoretical value of $Q_{W(\mathrm{SM})}^{p}\left(Q^{2}=0\right)=$ $0.0716 \pm 0.0008$ (indicated by the red star in Fig. 1.4), which has a relative uncertainty of $1.1 \%$. The large relative uncertainty of the global fit value and the difference between the global fit value and the theoretical value motivate a direct measurement of the $Q_{W}^{p}$ at low energy - the Q-weak experiment. With an anticipated uncertainty of $4.1 \%$, including the statistical and systematic uncertainties [34], the Q-weak experiment should improve the $Q_{W}^{p}$ measurement accuracy by about a factor of 7 .

\subsubsection{Electroweak Radiative Corrections}

When a particle interacts with various fields, the effective values of its physical properties, such as mass, charge and couplings may change due to radiative corrections. For example, an electromagnetic field produces virtual electron-positron pairs that change the distribution of charges and currents that generated the original electromagnetic field. This is a typical radiative correction, referred to as vacuum polarization [43]. Examples of vacuum polarization diagrams are shown in Fig. 1.5.

A renormalization scheme must be introduced in order to make higher order corrections finite by redefining some quantities. The values of physical observables may vary in different renormalization

schemes. In the following discussion, the renormalized $\sin ^{2} \hat{\theta}_{W}$ defined in the $\overline{\mathrm{MS}}$ scheme [7] is used. 

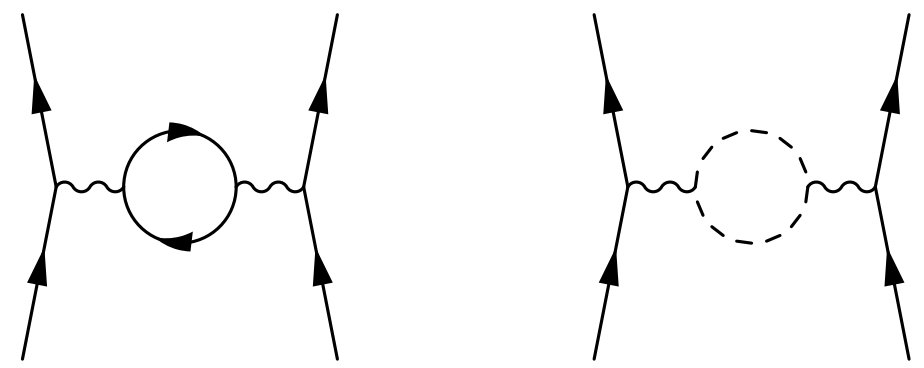

Figure 1.5: Typical one-loop self-energy diagrams, also known as vacuum polarization or oblique diagrams [7]. They represent the fermion-antifermion pair (left) and Higgs pair (right) coupling indirectly to the external fermions via virtual bosons.

Electroweak radiative corrections are higher order corrections involving the weak interaction in some way. Fig. 1.6 shows two representative $\gamma Z$ mixing loop and box Feynman diagrams that contribute to electroweak radiative corrections.
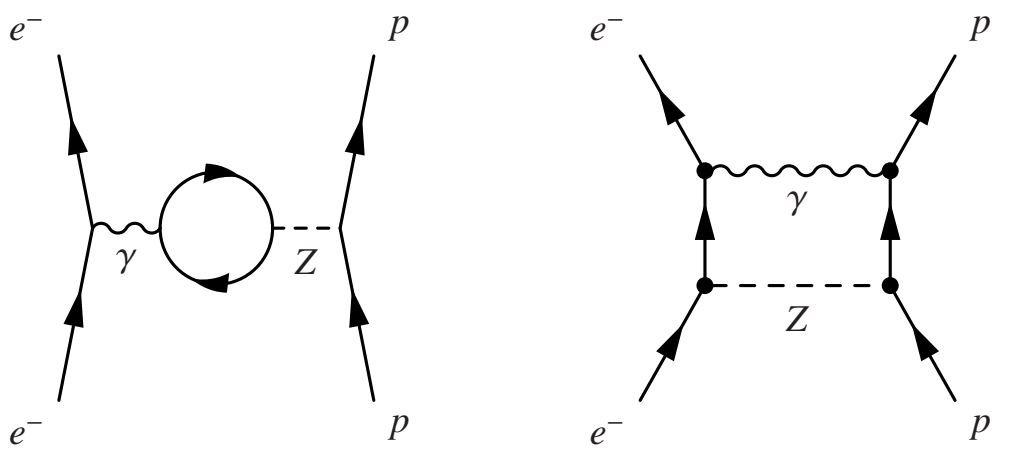

Figure 1.6: Typical $\gamma Z$ loop and box diagrams.

In 2003, J. Erler et al. expressed the proton's weak charge, including the higher-order contributions, in Ref. [15] as:

$$
Q_{W}^{p}=\left[\rho_{N C}+\Delta_{e}\right]\left[1-4 \sin ^{2} \hat{\theta}_{W}(0)+\Delta_{e}^{\prime}\right]+\square_{W W}+\square_{Z Z}+\square_{\gamma Z},
$$

in which $\rho_{N C}$ is a renormalization factor for the ratio of neutral to charged current interaction 
strength, $\Delta_{e}$ and $\Delta_{e}^{\prime}$ are the electron vertex and external leg corrections, $\square_{W W}$ and $\square_{Z Z}$ are the pure weak box graph corrections, and $\square_{\gamma Z}$ is the mixing gamma-Z box graph correction. With the following definitions and quantities $[9,15]$ :

$$
\begin{aligned}
& \alpha=(137.035999679 \pm 0.000000094)^{-1}, \\
& M_{Z}=(91.1876 \pm 0.0021) \mathrm{GeV} \\
& m_{e}=(0.510998910 \pm 0.000000013) \times 10^{-3} \mathrm{GeV} \\
& \hat{\alpha} \equiv \hat{\alpha}\left(M_{Z}\right)=(127.925 \pm 0.016)^{-1} \\
& \hat{s}^{2} \equiv 1-\hat{c}^{2} \equiv \sin ^{2} \hat{\theta}_{W}\left(M_{Z}\right)=0.231119 \pm 0.00014
\end{aligned}
$$

and an energy scale $(\Lambda)$ dependent constant $C_{\gamma Z}(\Lambda)$ given by [44]:

$$
C_{\gamma Z}\left(m_{\rho}\right)=3 / 2 \pm 1 \quad\left(\Lambda=m_{\rho} \simeq 0.77 \mathrm{GeV}\right)
$$

the radiative correction parameters in Eqn. 1.51 can be obtained as [15]:

$$
\begin{aligned}
& \rho_{N C}=1+\Delta \rho=1.0004, \\
& \Delta_{e}=-\frac{\alpha}{2 \pi}=-0.00116, \\
& \Delta_{e}^{\prime}=-\frac{\alpha}{3 \pi}\left(1-4 \hat{s}^{2}\right)\left[\ln \left(\frac{M_{Z}^{2}}{m_{e}^{2}}\right)+\frac{1}{6}\right]=-0.00142, \\
& \square_{W W}=\frac{7 \hat{\alpha}}{4 \pi \hat{s}^{2}}=0.01884, \\
& \square_{Z Z}=\frac{\hat{\alpha}}{4 \pi \hat{s}^{2} \hat{c}^{2}}\left(\frac{9}{4}-5 \hat{s}^{2}\right)\left(1-4 \hat{s}^{2}+8 \hat{s}^{4}\right)=0.00156, \\
& \square_{\gamma Z}=\frac{5 \hat{\alpha}}{2 \pi}\left(1-4 \hat{s}^{2}\right)\left[\ln \left(\frac{M_{Z}^{2}}{\Lambda^{2}}\right)+C_{\gamma Z}(\Lambda)\right]=0.00519 .
\end{aligned}
$$

The authors also included new higher-order terms in $\alpha$ and $\alpha_{s}$ and updated the analysis of nonperturbative, hadronic contributions. They reported the $\overline{\mathrm{MS}}$ scheme value for $\sin ^{2} \hat{\theta}_{W}$ at low energy 
as:

$$
\sin ^{2} \hat{\theta}_{W}(0)=0.23807 \pm 0.00017
$$

Based on this result, the Standard Model prediction of $Q_{W}^{p}$ (indicated by the red star in Fig. 1.4) was derived from Eqn. 1.51, as:

$$
Q_{W(\mathrm{SM})}^{p}=0.0716 \pm 0.0008 .
$$

The uncertainties and sources from this analysis are summarized in Table 1.3.

\begin{tabular}{lccl}
\hline \hline Sources & $\Delta Q_{W}^{p}$ & $\Delta Q_{W}^{p} / Q_{W}^{p}$ & \\
\hline $\sin ^{2} \hat{\theta}_{W}\left(M_{Z}\right)$ & \pm 0.0006 & $\pm 0.84 \%$ & (experimental uncertainty of $\sin ^{2} \theta_{W}$ at the Z-pole) \\
$\sin ^{2} \hat{\theta}_{W}(0)_{\text {hadronic }}$ & \pm 0.0003 & $\pm 0.42 \%$ & (hadronic contributions to the running of $\left.\sin ^{2} \theta_{W}\right)$ \\
$W W$-box, ZZ-box & \pm 0.0001 & $\pm 0.14 \%$ & (strong corrections to $W W$-box and $Z Z$-box graphs) \\
$\gamma Z$-box & \pm 0.0005 & $\pm 0.70 \%$ & (strong corrections to $\gamma Z$-box graphs) \\
Charge symmetry & 0 & $0 \%$ & (isospin-breaking effects in nucleon current matrix) \\
\hline Total & \pm 0.0008 & $\pm 1.12 \%$ & \\
\hline \hline
\end{tabular}

Table 1.3: Uncertainties and their sources for the Standard Model prediction of $Q_{W}^{p}$ in the $\overline{\mathrm{MS}}$ scheme [15].

For e-p scattering at Q-weak kinematics, besides the primary $\gamma Z$ mixing one-loop electroweak radiative corrections, which have been well studied, one of the most important radiative corrections for precision electroweak measurements is from $\gamma Z$ box diagrams. In 2009, M. Gorchtein and C. J. Horowitz demonstrated via dispersion theory that the $\gamma$ Z-box corrections in forward kinematics were not suppressed by the small weak charge of electron, suggesting that the theoretical uncertainty for Q-weak might be substantially underestimated [45]. More refined calculations were performed independently by several groups, and the results are summarized in Table 1.4. The groups tend to agree that the size of the $\gamma Z$-box correction to $Q_{W}^{p}$ is $0.0057(\sim 8 \%)$, though they tend to disagree on the size of a conservative uncertainty to assign to the correction, which ranges from \pm 0.0008 to 
$\pm 0.0020( \pm 1.1 \%$ to $\pm 2.8 \%)$.

\begin{tabular}{|c|c|c|c|}
\hline PV Amplitude & $\begin{array}{l}\text { Corrections to } Q_{W}^{p} \\
\text { at } E=1.165(\mathrm{GeV})\end{array}$ & Authors & Reference \\
\hline \multirow{4}{*}{$\begin{array}{c}A(e) \times V(p) \\
(\text { vanishes as } E \rightarrow 0)\end{array}$} & $0.0026 \pm 0.0026$ & Gorchtein \& Horowitz & {$[45]$} \\
\hline & $0.0047_{-0.0004}^{+0.0011}$ & $\begin{array}{l}\text { Sibirtsev, Blunden, } \\
\text { Melnitchouk,Thomas }\end{array}$ & {$[46]$} \\
\hline & $0.0057 \pm 0.0009$ & Rislow \& Carlson & {$[47]$} \\
\hline & $0.0054 \pm 0.0020$ & $\begin{array}{l}\text { Gorchtein, Horowitz } \\
\text { and Ramsey-Musolf }\end{array}$ & {$[48]$} \\
\hline \multirow[t]{2}{*}{$\begin{array}{c}V(e) \times A(p) \\
(\text { finite as } E \rightarrow 0)\end{array}$} & $0.0052 \pm 0.0005$ & $\begin{array}{c}\text { Marciano and Sirlin } \\
\text { (as updated by Erler, Kurylov } \\
\text { and Ramsey-Musolf) }\end{array}$ & $\begin{array}{l}{[49]} \\
{[15]}\end{array}$ \\
\hline & $0.0037 \pm 0.0004$ & $\begin{array}{c}\text { Blunden, Melnitchouk, } \\
\text { and Thomas }\end{array}$ & {$[50]$} \\
\hline
\end{tabular}

Table 1.4: $\gamma Z$-box corrections near Q-weak kinematics.

\subsection{Constraints on the Standard Model Parameters and New Physics}

\subsubsection{Constraints on Quark Vector Couplings}

As discussed previously, the quark weak couplings, $C_{1 f}$ and $C_{2 f}$, may be determined experimentally, as they are related to $\sin ^{2} \theta_{W}$ in the SM. Elastic scattering measurements constrain the vector couplings $C_{1 f}$. Fig. 1.7 shows the present experimental constraints on the vector couplings of u-, d-quarks, as well as the anticipated knowledge after the Q-weak measurement. The precise atomic PV measurements and other previous experiments set up the primary constraints on the combination of $C_{1 u}+C_{1 d}$, and the recent high precision PVES scattering measurements provide an essentially orthogonal constraint on the combination of $C_{1 u}-C_{1 d}$. The Q-weak measurement is expected to place dramatically tighter constraints on the couplings. 


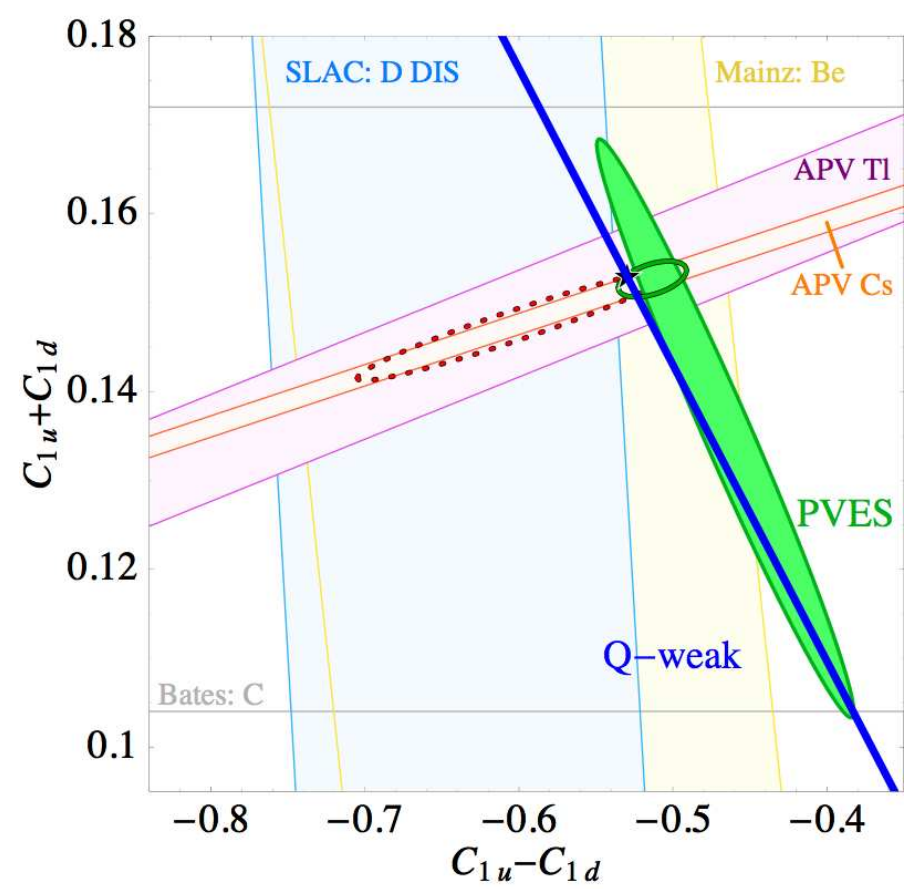

Figure 1.7: Constraints on the weak vector couplings for u-, d-quarks [41]. The dotted ellipse represents the constraint from the combination of atomic parity violation experiments and the previous experimental limits, including APV Cs [17], APV TI [51], MIT-Bates C [35] and SLAC Deuterium [52], Mainz Be [53] measurements. The solid filled green ellipse denotes the high precision PVES experiments on hydrogen, deuterium and helium targets, including HAPPEx [36], G0 [37], PVA4 [38, 39], SAMPLE [14]. The solid green contour indicates the full constraint obtained by combining all results. The black star indicates the SM predication. The projected Q-weak $4 \%$ measurement, assuming agreement with the SM, will impose a narrow blue band (the anticipated uncertainty band) onto the phase space plot.

\subsubsection{Example of Model-independent Constraints}

The e-p elastic scattering process can be described by a low-energy effective four-point interaction between electrons and quarks [7]. Considering the electron-quark weak neutral current interaction in the form of $A(e) \times V(q)$, an effective interaction Lagrangian for parity-violation in potential new physics, for example new sources of parity violation between electrons and light quarks, can be 
expressed in terms of an effective contact interaction as [15]:

$$
\mathcal{L}_{N E W}^{P V}=\frac{g^{2}}{4 \Lambda^{2}} \bar{e} \gamma_{\mu} \gamma_{5} e \sum_{q} h_{V}^{q} \bar{q} \gamma^{\mu} q
$$

where $g$ represents the coupling strength, $\Lambda$ is the characteristic mass scale, and the quark-specific coefficients $h_{V}^{q}$ are couplings associated with possible new physics, resulting in a modification of the e-q coupling. In analogy with Fermi theory, here, the new physics could be, for example, an additional neutral gauge boson $Z^{\prime}$, much as in beta-decay, the effective Fermi interaction was eventually discovered to be due to heavy charged gauge bosons. This is why $\Lambda$ represents the energy scale of the new physics.

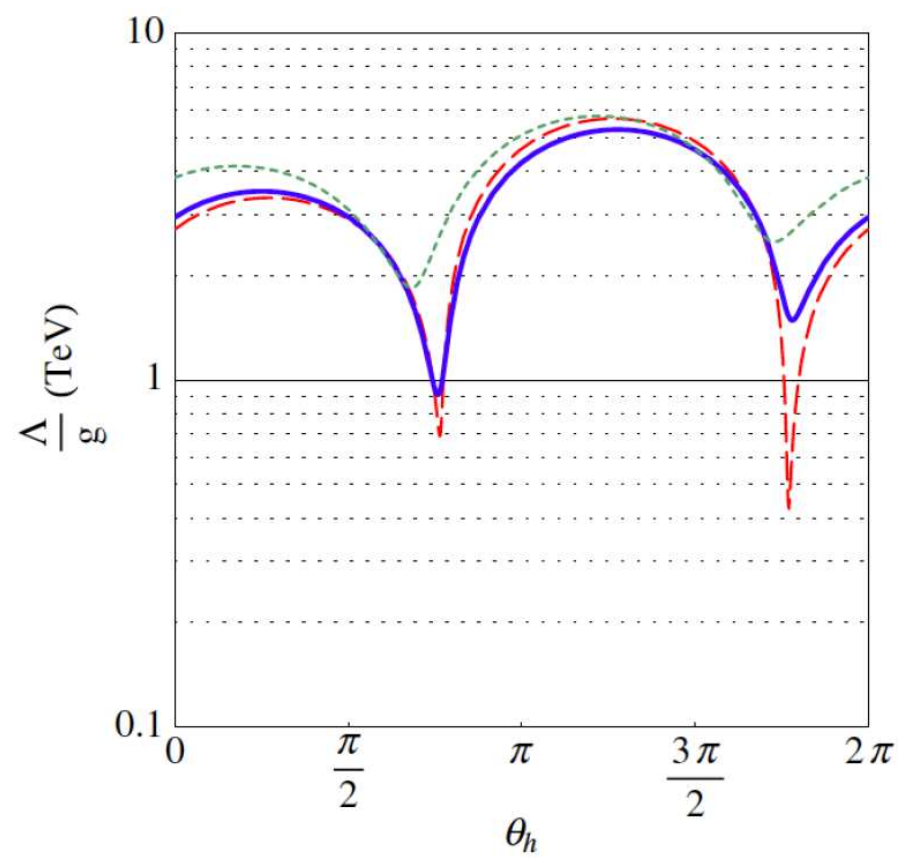

Figure 1.8: Model independent mass limits [41] - the bounds on the size of the interaction represented by Eqn. 1.66. The long-dashed red curves shows the limit $(\Lambda / g>0.4 \mathrm{TeV})$ without parityviolating electron scattering data (atomic PV only). The solid blue curve shows the limit $(\Lambda / \mathrm{g}>$ $0.9 \mathrm{TeV}$ ) including parity-violating electron scattering experiment. The short-dashed green curve indicates the reach of the $\mathrm{Q}$-weak experiment $(\Lambda / \mathrm{g}>2 \mathrm{TeV})$ assuming agreement with the Standard Model is observed. 
Fig. 1.8 shows experimental constraints on the parameters of the interaction Lagrangian in Eqn. 1.66. Referring to the figure, the angle $\theta_{h}$ is a flavor mixing angle of the new physics: $h_{V}^{u}=\cos \theta_{h}$ and $h_{V}^{d}=\sin \theta_{h}$. New physics is ruled out at the $95 \%$ confidence level below the curves. The Q-weak measurement would improve the lower limit on the energy scale to coupling ratio $(\Lambda / g)$ to $\sim 2 \mathrm{TeV}$ in this analysis.

\subsubsection{Model-dependent Constraints}

Many studies have been performed to address different scenarios of new physics that the Q-weak experiment may impact. In these studies, several models were considered as the possible extensions of the SM, such as Supersymmetry (SUSY) Loops in the Minimal Supersymmetric Standard Model (MSSM), $E_{6} Z^{\prime}$ extra neutral gauge interaction, R-parity-violating (RPV) SUSY, Leptoquarks.

Different experiments have different sensitivities to the new physics extensions, and they are complementary to each other. For example, the Q-weak measurement is sensitive to scalar leptoquarks, while a purely leptonic experiment like E158 is not [54]. Discussions of different SM extensions are beyond the scope of this thesis work; a review is given in Ref. [15].

\subsection{Summary}

The weak vector coupling of the proton is sensitive to physics beyond the SM. This motivates a precision experiment to use the proton's weak charge as a probe for new physics beyond the SM. The proton's weak charge can be determined by measuring the parity-violating asymmetry of elastic e-p scattering. Since the asymmetry is very small, measuring it to high precision requires special experimental techniques, discussed in the next chapter. 


\section{Chapter 2}

\section{The Q-weak Experiment}

\subsection{Overview}

The Q-weak experiment is being carried out at Thomas Jefferson National Accelerator facility (Jefferson Lab, or JLab) [2] in Newport News, Virginia, USA. The goal of the Q-weak experiment is to determine the proton's weak charge to $4.1 \%$ combined systematic and statistical uncertainties (see Table 2.1 for anticipated uncertainties). This will be done by measuring the PV asymmetry $(\sim 230 \mathrm{ppb})$ to $\pm 2.5 \%$. The systematic uncertainties are mainly constrained by the experimental apparatus and beam conditions. In order to meet the final goal, the statistical uncertainty has to be small $(<2.1 \%)$, implying that enough running time ( $\sim 2500$ hours) and high luminosity (more than $150 \mu \mathrm{A}$ beam current) are required. The experiment was first proposed in 2001 [55], and was under construction and development from 2002 - 2009. The installation of experimental apparatus in experimental Hall C began in November, 2009. After commissioning, the Q-weak experiment entered its first production data-taking phase in January, 2011 and completed it in May, 2011. Data collection is anticipated during phase II from November, 2011 through May, 2012, to achieve the final experimental goal. The results presented in this work are based on the commissioning and phase I data.

The Q-weak experiment is designed to measure the parity-violating asymmetry to high precision 


\begin{tabular}{lll} 
Source of uncertainty & $\Delta \mathrm{A}_{\text {phys }} / \mathrm{A}_{\text {phys }}$ & $\Delta \mathrm{Q}_{\mathrm{W}}^{\mathrm{p}} / \mathrm{Q}_{\mathrm{W}}^{\mathrm{p}}$ \\
\hline Statistical $(2544$ hours at $150 \mu \mathrm{A})$ & $2.1 \%$ & $3.2 \%$ \\
\hline Systematic: & & $2.6 \%$ \\
Hadronic structure corrections & - & $1.5 \%$ \\
Beam polarimetry & $1.0 \%$ & $1.5 \%$ \\
Absolute $Q^{2}$ determination & $0.5 \%$ & $1.0 \%$ \\
Helicity-correlated beam properties & $0.5 \%$ & $0.7 \%$ \\
Backgrounds & $0.5 \%$ & $0.7 \%$ \\
\hline Total & $2.5 \%$ & $4.1 \%$ \\
\hline
\end{tabular}

Table 2.1: The anticipated experimental uncertainties in the Q-weak experiment for both the physics asymmetry and the extracted $\mathrm{Q}_{\mathrm{W}}^{\mathrm{p}}[54]$.

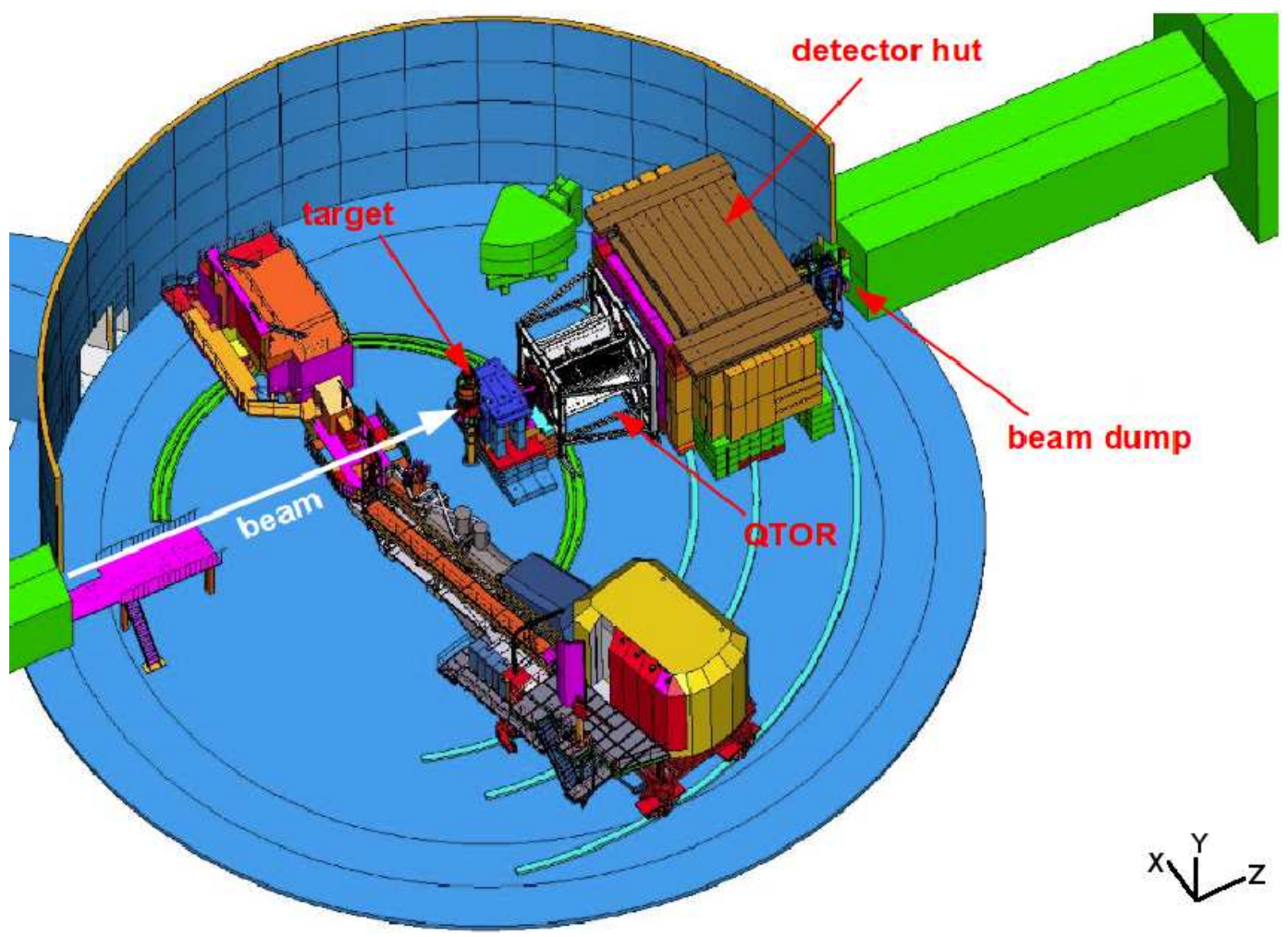

Figure 2.1: 3D model of the Q-weak layout in Hall C at JLab [1]. The electron beam travels from left to right, going through the target, QTOR spectrometer and detector hut.

in the elastic scattering of longitudinally polarized electrons from an unpolarized liquid hydrogen target at low momentum transfer and at very forward angles. Fig. 2.1 displays a 3D model of the 


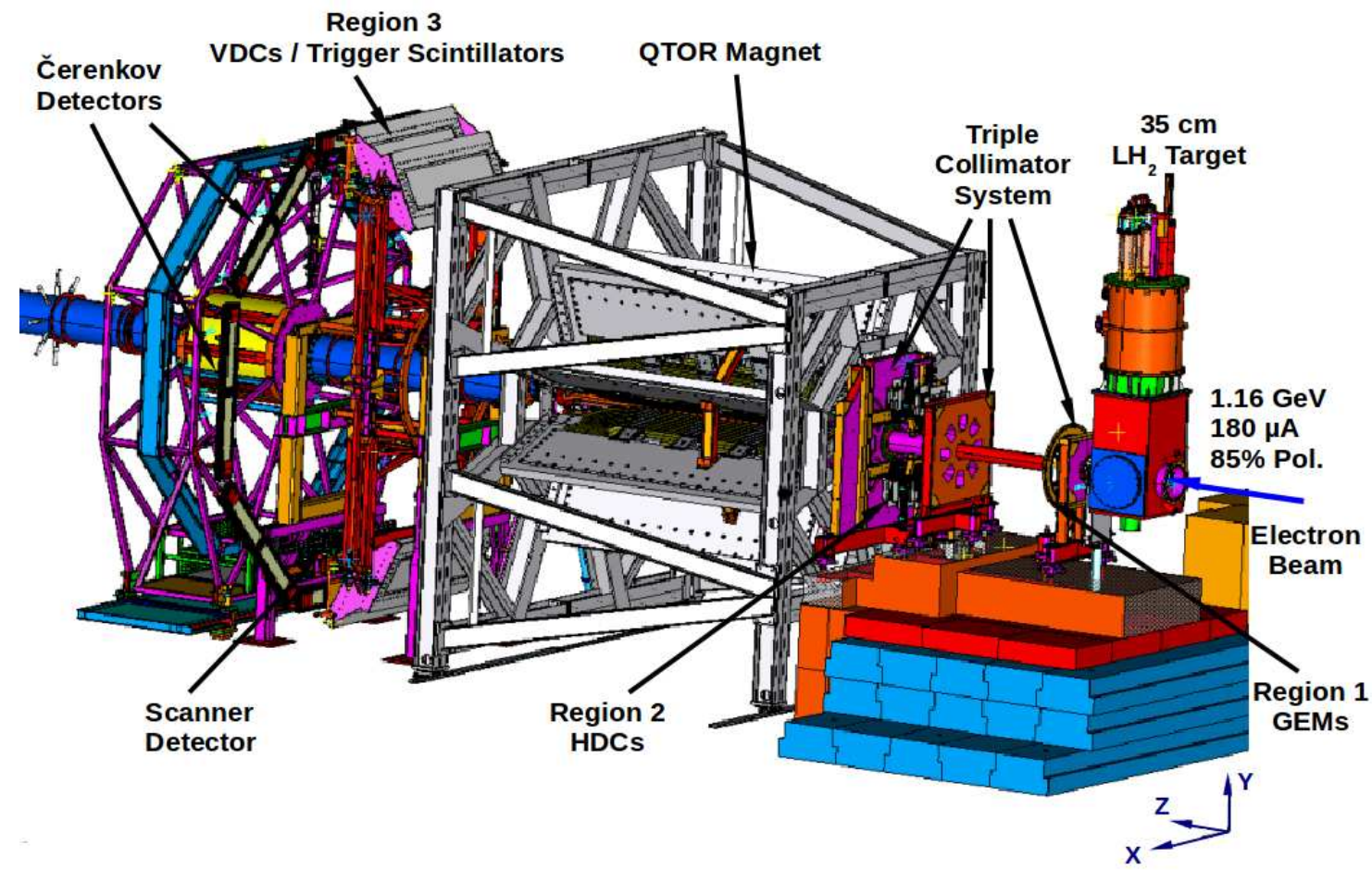

Figure 2.2: An overview of the Q-weak experimental setup [1]. The longitudinally polarized electron beam impinges upon the $\mathrm{LH}_{2}$ target. After selection by a triple collimator system, the elastically scattered electrons can be focused by the QTOR spectrometer onto its focal plane and detected by a set of quartz Čerenkov detectors. The tracking chambers (Region 1 GEMs, Region 2 HDCs and Region 3 VDCs), which are designed to measure $Q^{2}$ in calibration mode (sub-nA) only, are retracted during the high current $A_{P V}$ measurement.

Q-weak setup in Hall C, and Fig. 2.2 illustrates schematically the basic apparatus (the Q-weak coordinate systems are defined in Section 4.2). The $1.162 \mathrm{GeV}$ electron beam, longitudinally polarized in excess of $85 \%$, impinges on a $35 \mathrm{~cm}$ long liquid hydrogen target. Scattered electrons with $\sim 8^{\circ}$ scattering angle are selected by the subsequent collimator system, and enter the Q-weak toroidal (QTOR) magnetic spectrometer. Within the magnetic field, the elastically scattered electrons are deflected an additional $10^{\circ}$, and are focused onto a set of eight quartz Čerenkov bars. Čerenkov light generated by the scattered electrons is transported by total internal reflection to photo-multiplier tubes (PMTs) located at either end of each bar. To achieve the very high statistical precision needed for Q-weak, the main detectors are operated in current mode. 
High resolution tracking detectors, indicated by Region 1 Gas Electron Multiplier (GEMs), Region 2 Horizontal Drift Chamber (HDCs) and Region 3 Vertical Drift Chamber (VDCs) in Fig. 2.2, are designed to be used in dedicated low current (sub-nA) counting-mode calibration runs for $Q^{2}$ determination and background studies. During high-current parity runs, these chambers are retracted from the spectrometer.

\subsection{The Polarized Electron Beam}

\subsubsection{CEBAF Accelerator}

The CEBAF (Continuous Electron Beam Accelerator Facility) accelerator at JLab can deliver essentially continuous electron beams ${ }^{1}$ of up to $6 \mathrm{GeV}$, shared by three experimental halls simultaneously. A schematic layout of the accelerator is presented in Fig. 2.3. Polarized electron beams generated in the injector are accelerated by a pair of recirculating superconducting RF linear accelerators (LINACs) linked by two arcs, and delivered to different experimental areas by the beam switchyard. The $45 \mathrm{MeV}$ electron beams from the injector can travel through the racetrack up to five times, gaining up to $550 \mathrm{MeV}$ per LINAC. The separate five-pass beam orbits in the arcs make it possible to accommodate variable beam energies to different halls by selecting a different number of passes. To obtain the required Q-weak beam energy of $1.162 \mathrm{GeV}$, Hall $\mathrm{C}$ uses single pass electron beam.

In the injector region, the polarized electron beams are generated via the photoelectric effect induced by a polarized laser beam incident on a GaAs crystal. The beam for each hall is created by its own individual laser source and is separated from the beams for other halls by timing.

To achieve high experimental statistics, both high beam polarization and high beam intensity are required for Q-weak. Beam currents up to about $200 \mu \mathrm{A}$ can be provided by the accelerator. However, limited by the cooling power available for the cryogenic targets in Hall A and Hall C, high current can not be reached simultaneously in both of these halls. After an extensive commissioning

\footnotetext{
${ }^{1}$ In fact, CEBAF uses superconducting accelerating cavities at $1497 \mathrm{MHz}$; each hall receives an electron pulse train at $499 \mathrm{MHz}$ repetition frequency.
} 


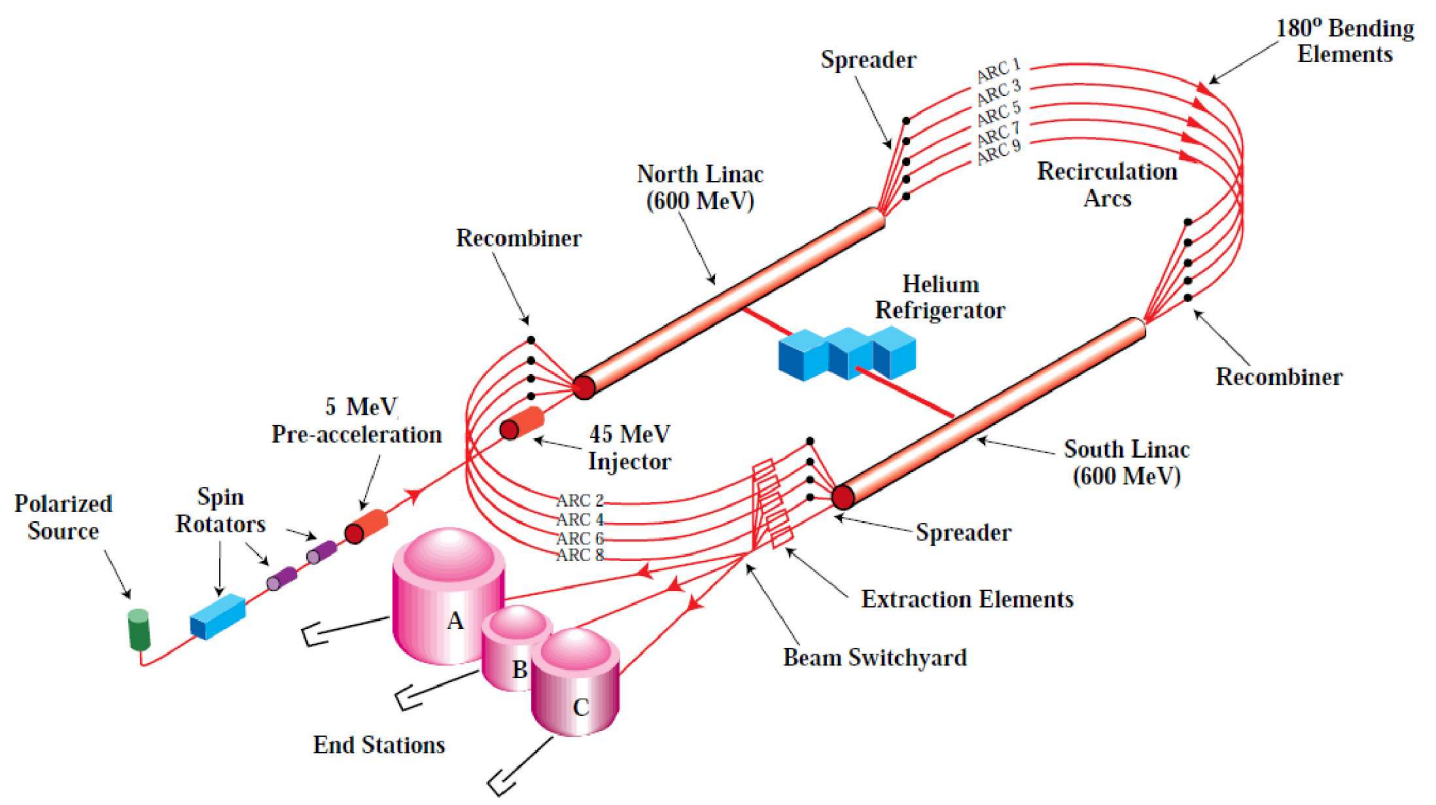

Figure 2.3: Schematic layout of the CEBAF accelerator [56]. The positions of injector, North and South LINACs and the five-pass arcs are shown, along with the positions of the three experimental halls currently in use.

process, it was demonstrated that $180 \mu \mathrm{A}$ could be delivered to Hall C for the Q-weak experiment.

\subsubsection{The Polarized Electron Source}

The longitudinally polarized electron beam for Q-weak is produced by photoemission from gallium arsenide (GaAs) in a DC high voltage photogun $[57,58,59]$. The schematic layout of the polarized source is shown in Fig. 2.4. Circularly polarized photons generated by a laser system are directed onto a photocathode of strained-superlattice GaAs [60], leading to highly polarized electrons liberated by the photoelectric effect. The potential difference between the source and the injector extracts the electrons into the injector, and then into the accelerator. The polarization direction of emitted electrons is given by the circular polarization axis of the laser light, and thus can be reversed by alternating the handedness of the photons impinging on the GaAs crystal. By using a strained-superlattice GaAs photocathode, the beam for the Q-weak experiment can obtain an average polarization greater than $85 \%$ (see Section 6.5.2). 
polarized source

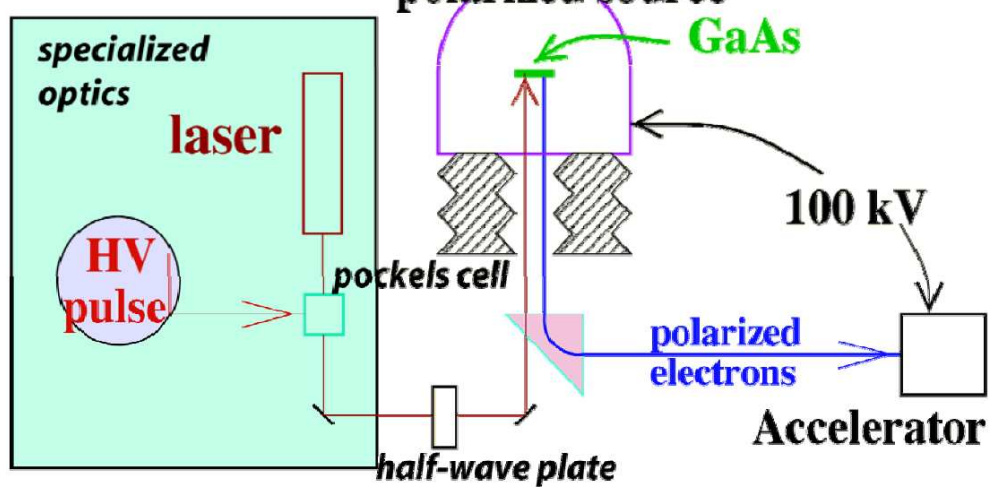

Figure 2.4: The schematic layout of the polarized source [61].

\section{Beam Helicity Control}

Several optical elements are used for laser control at the polarized source. One key component is a helicity Pockels Cell (HPC). The Pockels Cell is a birefringent crystal whose indices of refraction vary with applied electric fields. By varying the high voltage applied to the HPC, linearly, circularly or elliptically polarized light can be selected. The HPC serves as a quarter-wave plate to produce left-handed or right-handed, circularly polarized light (LCP or RCP light) for electron beam helicity control.

The helicity of the Q-weak electron beam is reversed by changing the polarity of the high voltage applied to the HPC at a frequency of $960 \mathrm{~Hz}$. Each $1 \mathrm{~ms}$ is referred to as a macro-pulse (MPS), during which the helicity state is a constant. In between MPS windows, there is a $70 \mu$ s settling time used to allow the high voltage on the HPC to stabilize.

In order to reduce the noise from beam property drifts, a "quartet" pattern in the sequence of " $+--+"$ or " $-++-"$ is used in generating the electron's helicity, with the first bit selected from a pseudo-random sequence. Asymmetries are calculated for each quartet and are histogrammed. Averaging the results gives the measured asymmetry. Delayed helicity reporting (with 8-quartet delay) to the data acquisition system is used to eliminate the potential cross-talk between detector 
signals and the helicity state in the injector. For further systematic error control, an Insertable Half Wave Plate (IHWP) is periodically ( every 24 hours) inserted or removed from the laser path to reverse the direction of the beam helicity. Asymmetry data are acquired either with IHWP "IN" or "OUT" (inserted or retracted) to search for false asymmetries due to systematic effects.

\section{Helicity-correlated Beam Property Control}

Good control of helicity-correlated beam properties is necessary to minimize systematic effects. The helicity-correlated beam property specifications for Q-weak are listed in Table 2.2. Ideally, there should be no beam property change under helicity reversal. However, in practice, the laser beam and the Pockels Cell effects may lead to helicity-correlated intensity, position and angle differences on the GaAs crystal. The systematic responses are then transferred to the polarization of the emitted electron beam, leading to a polarization induced transport asymmetry (PITA).

A number of techniques are employed to control the helicity-correlated beam properties. Helicitycorrelated beam intensity changes are signified by the charge asymmetry $A_{q}$, defined as the relative

difference of beam charge delivered in different helicity states: $A_{q}=\frac{Q^{+}-Q^{-}}{Q^{+}+Q^{-}}$. To control $A_{q}$, a charge feedback system is adopted. In the feedback loop, $A_{q}$ is measured with the beam charge monitors (BCMs) in Hall C. The results are then used to determine a new voltage applied to the Pockels Cell to null $A_{q}$. Similarly, a PZT (a mirror attached to a Piezo-electric transducer, with the angle of the mirror being changed by application of an AC voltage at low frequency) feedback loop is commissioned to null out the helicity correlated beam position differences $(\delta x, \delta y)$ at the target to the $\mathrm{nm}$ level.

\section{Spin Precession}

The polarization of the electron beam at the source is either aligned or anti-aligned with the electron's momentum. However, the spin orientation precesses relative to the momentum as the beam is transported through the bending dipoles in accelerator arcs and end station transport lines. To obtain longitudinally polarized beam at the experimental target, the spin orientation of the electron beam is 


\begin{tabular}{l|ll}
\hline \hline Parameter & 2544 hours & $8 \mathrm{~ms}$ \\
\hline Beam Intensity & $A_{q}<10^{-7}$ & $<3 \times 10^{-4}$ \\
Beam Energy & $\Delta E / E \leqslant 10^{-9}$ & $\Delta E / E \leqslant 3 \times 10^{-6}$ \\
Beam Position & $\langle\delta x\rangle<2 \mathrm{~nm}$ & $7 \mu \mathrm{m}$ \\
Beam Angle & $\langle\delta \theta\rangle<30 \mathrm{nrad}$ & $100 \mu \mathrm{rad}$ \\
Beam Diameter & $\langle\delta \sigma\rangle<0.7 \mu \mathrm{m}$ & $<2 \mathrm{~mm}$ \\
\hline \hline
\end{tabular}

Table 2.2: Summary of systematic error requirements on helicity-correlated beam parameters for the entire experiment or a quartet spin cycle [54]. The helicity-correlated differences in beam position $\Delta x$ and $\Delta y$, beam direction $\Delta \theta_{x}$ and $\Delta \theta_{y}$, beam energy $\Delta E$ and beam intensity $A_{q}$ are continuously measured during $\mathrm{Q}$-weak data taking.

manipulated in the source with Wien filters [62, 63, 64], which serve as spin rotators to compensate for the spin precession.

A Wien filter consists of homogeneous electric and magnetic fields $(\vec{E}$ and $\vec{B})$, which are perpendicular to each other and transverse to the direction of travel of the electrons. The $\vec{E}$ and $\vec{B}$ field strengths are adjusted so that the electrons experience zero net force as they travel through the crossed fields, while rotating the electron spin vector about the magnetic field axis.

The net rotation angle of the spin vector, denoted by the Wien angle $\left(\eta_{\text {wien }}\right)$, is adjusted and set by maximizing the longitudinal polarization measured in the experimental hall. The slow helicity reversal status of the experiment is determined by the IHWP together with the Wien angle settings.

\subsubsection{Beam Polarimetry}

The Q-weak experiment requires knowledge of the electron beam polarization to $1 \%$. The beam polarization is measured by two complementary devices: the Hall $\mathrm{C}$ Møller polarimeter and the Compton polarimeter. 


\section{Møller Polarimeter}

The beam polarization is measured periodically using the Hall $\mathrm{C}$ Møller polarimeter $[65,66]$. The measurement is based on the spin-dependent cross-section asymmetry $A_{M}$ in the process of elastic scattering of polarized electrons from polarized electrons $\vec{e}+\vec{e} \longrightarrow e+e$, given by:

$$
A_{M}=\frac{\left(\frac{d \sigma}{d \Omega}\right)^{\uparrow \uparrow}-\left(\frac{d \sigma}{d \Omega}\right)^{\uparrow \downarrow}}{\left(\frac{d \sigma}{d \Omega}\right)^{\uparrow \uparrow}+\left(\frac{d \sigma}{d \Omega}\right)^{\uparrow \downarrow}}=P_{b} P_{t} A_{z z},
$$

where $\uparrow \uparrow$ and $\uparrow \downarrow$ refer to the direction of beam and target polarization, and the analyzing power is given by

$$
A_{z z}(\theta)=-\frac{\sin ^{2} \theta\left(8-\sin ^{2} \theta\right)}{\left(4-\sin ^{2} \theta\right)^{2}}
$$

and $\theta$ is the center of mass $(\mathrm{CM})$ scattering angle. Knowing the target polarization $P_{t}$, the measured $A_{M}$, and the kinematics of scattering, the beam polarization $P_{b}$ can be determined. The analyzing power $A_{z z}$ has a maximum value of $-\frac{7}{9}$ at a $\mathrm{CM}$ scattering angle of $\theta=90^{\circ}$. This corresponds to a scattering angle in the lab frame $\theta_{\text {lab }}=1.83^{\circ} / \sqrt{E(\mathrm{GeV})}$, where $E$ represents the incident beam energy. This defines the best kinematics at which to perform the measurements.

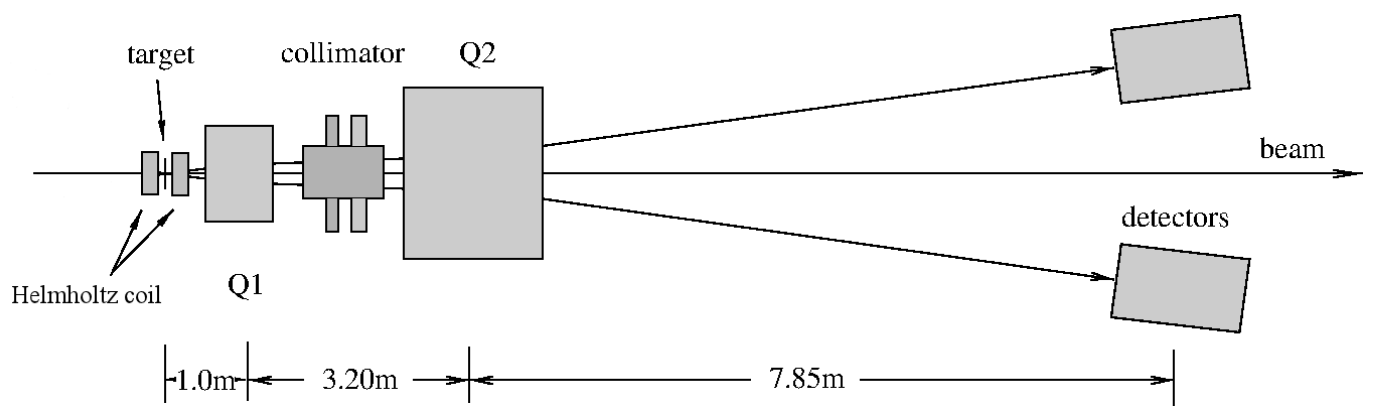

Figure 2.5: The layout of Hall C Møller polarimeter [54, 1]. A superconducting solenoid is used to drive a pure iron target foil into saturation. Both the magnetic field and the foil are oriented perpendicular to the beam. This results in target electrons polarized parallel (or anti-parallel) to the electron beam direction.

Fig. 2.5 shows a schematic diagram of the Møller apparatus. During Q-weak measurements, 
the polarized target electrons are provided by a pure iron foil of $1 \mu \mathrm{m}$ thickness, which is driven into saturation with a $3.5 \mathrm{~T}$ superconducting solenoid. Target electrons are polarized parallel or anti-parallel to the electron beam direction and with a polarization known to better than $0.5 \%$. The preferred kinematics are defined by the spectrometer magnets Q1 and Q2 and the collimator between them. Elastically scattered Møller electrons with $\sim 90^{\circ} \mathrm{CM}$ scattering angles are focused onto the detectors and are detected in coincidence. The Møller asymmetry, obtained by comparing the two beam helicity states, is used to extract the beam polarization.

The polarimeter is restricted to low beam currents $(<8 \mu \mathrm{A})$ due to beam-heating effects which may result in the depolarization of the iron foil target. Polarization measurements are made weekly in dedicated runs at low current. Typically, a statistical uncertainty of $0.4 \%$ and a systematic uncertainty of $0.6 \%$ [65] are achieved in a $\sim 1$ hour run.

\section{Compton Polarimeter}

A new Compton polarimeter developed for the Q-weak experiment [67] is used as an independent polarization measurement. Because it does not require insertion of a solid target or retuning of the beam, it permits non-invasive, continuous measurements to be conducted in parallel with production runs. The principle of the measurement is based on the polarization sensitivity of electron-photon

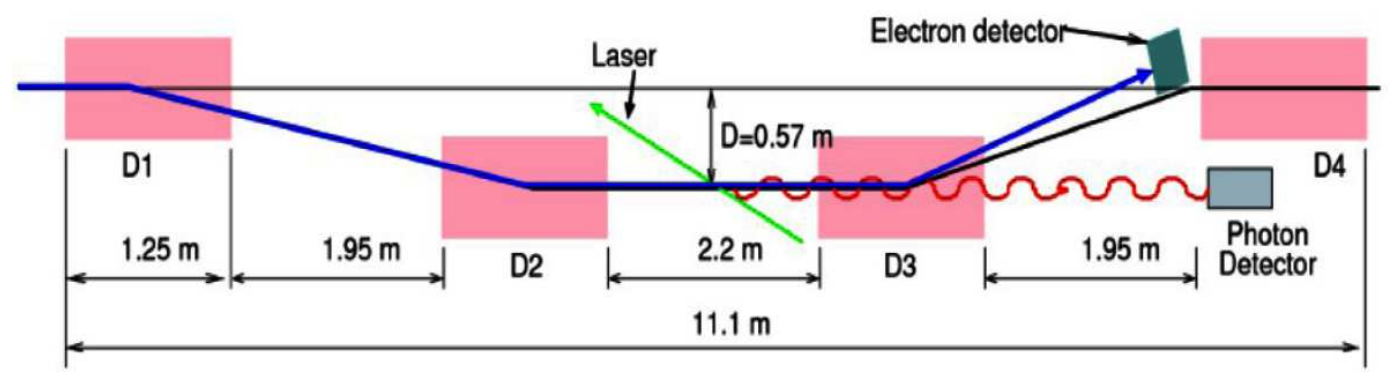

Figure 2.6: The schematic diagram of the Compton polarimeter [1].

scattering. A schematic diagram of the Compton polarimeter is shown in Fig. 2.6. The electron beam interacts with circularly polarized laser light provided by a high power green laser system, 
and the polarization dependent feature of the Compton cross-section is used to determine the beam polarization. The laser system consists of a $10 \mathrm{~W} \mathrm{CW} \mathrm{(Continuous} \mathrm{Wave)} \mathrm{green} \mathrm{laser} \mathrm{with} \mathrm{a} \mathrm{wave-}$ length of $532 \mathrm{~nm}$ and a low-gain resonant cavity used to lock the laser. With the cavity gain of about 100 , the stored laser power is approximately $700-800$ Watts. Four dipoles are used to divert the electron beam through a magnetic chicane to separate the scattered and unscattered electrons. Backscattered photons are measured by a photon detector. The angular distribution of scattered electrons is measured by a diamond micro-strip detector.

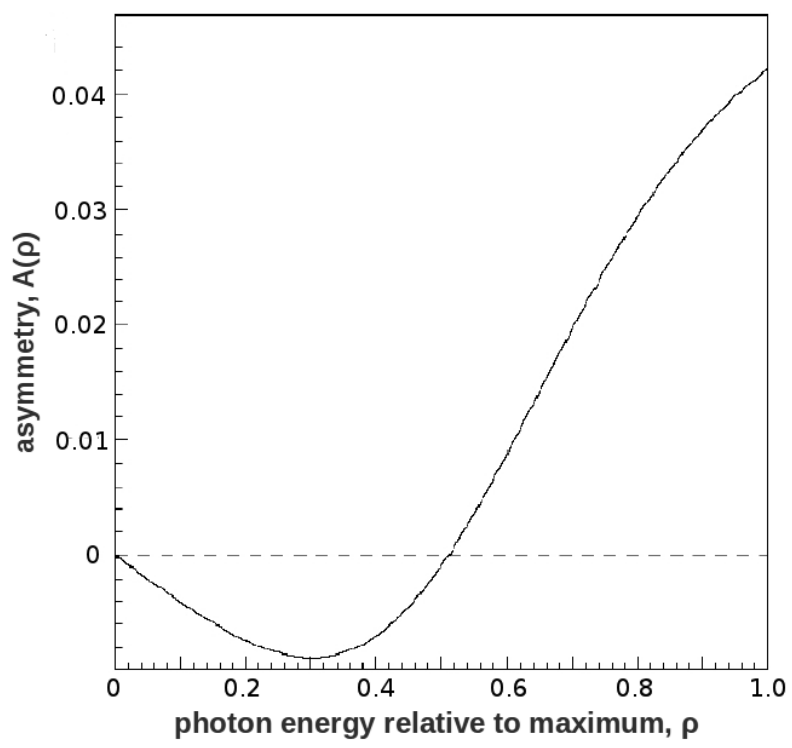

Figure 2.7: Theoretical Compton scattering asymmetry versus energy of back scattered photons [68]. For Q-weak kinematics, with $1.162 \mathrm{GeV}$ electron beam and $2.325 \mathrm{eV}$ laser light, the Compton edge energy is $46 \mathrm{MeV}$, corresponding to $\rho=1$ on the horizontal scale.

The theoretical Compton cross-section asymmetry is well-known in Quantum Electrodynamics (QED), as discussed in Ref. [69]. A plot of this function versus energy of the scattered photons is shown in Fig. 2.7. The maximum energy is given by a kinematic limit $E_{\max }$, known as the Compton edge. The asymmetry crosses zero when the scattered photon energy is about half of the maximum energy $\left(E=0.512 E_{\max }\right)$, and rises to $A_{\max }=0.0421$ at the Compton edge. By mapping the microstrip detector position onto electron momentum, and hence the photon recoil energy, the scattered 
electron asymmetry can be fitted against the theoretical asymmetry function to determine the beam polarization. Typically, a statistical uncertainty of $<1 \%$ per hour with an anticipated systematic uncertainty of $<1 \%$ [65] is achievable (see Section 6.5.2).

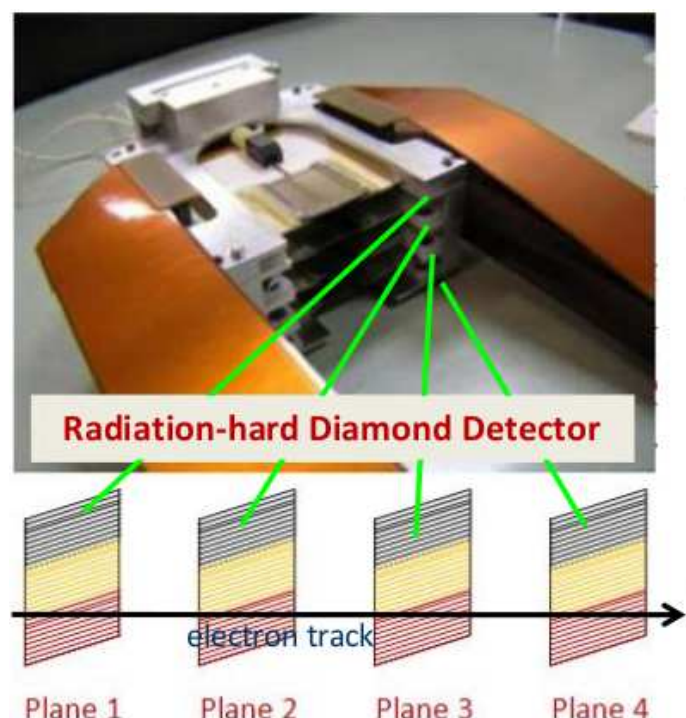

Figure 2.8: The Compton multi-strip diamond electron detector [1]. This electron detector consists of four $21 \mathrm{~mm} \times 21 \mathrm{~mm}$ planes of diamond, with $1 \mathrm{~cm}$ distance in between. There are 96 horizontal $200 \mu \mathrm{m}$ wide strips on each plane. It is located $5 \mathrm{~mm}$ from the main beam for position determination of the incident electrons, from which the momentum can be deduced.

The utilization of a photon detector and an electron detector in the Compton polarimeter allows two independent measurements of the beam polarization, and the coincidence between these two detectors in principle provides useful information for calibration of the photon detector.

The photon detector consists of a large scintillating crystal coupled to a PMT, operated as an electromagnetic calorimeter. During Q-weak commissioning, a single undoped CsI (Cesium Iodide) crystal was used. However, it was found to exhibit phosphorescence; the long time "afterglow" in this material results in history-dependent pedestal shifts in its output spectrum. A GSO (Germanium Silicon Oxide) crystal borrowed from Hall A Compton was used for about one month during Run I, before changing to use a $\mathrm{PbWO}_{4}$ (Lead Tungstate) crystal in the remaining measurements from late 
April of 2011 [70].

Recoil electrons are deflected from the primary electron beam by the third dipole of the Compton chicane. By measuring the spatial displacement of recoil electrons from the main beam, the energy of the scattered photon may be deduced. A CVD (Chemical Vapor Deposition) diamond micro-strip detector is used for this purpose, as illustrated in Fig. 2.8. The detector consists of four $21 \mathrm{~mm} \times 21$ mm planes with 96 horizontal $200 \mu \mathrm{m}$ wide strips on each. The fine strip pitch on this detector allows for good momentum resolution. The detector initially suffered from poor efficiency mainly caused by poor grounding of the readout boards, resulting in excess noise experienced in the Hall C tunnel. This necessitated increasing the detector thresholds. Nonetheless, percent-level beam polarization measurements were made in hour long runs during Run I [71]. Following redesign and upgrade of the readout electronics during summer 2011, the performance was dramatically improved.

\subsubsection{Beam Monitors}

A variety of devices throughout the accelerator are employed to monitor the electron beam. There are about 40 monitors currently in use along the Hall $\mathrm{C}$ beam line [72]. In order to reduce systematic errors arising from helicity correlated beam properties, the accelerator machine conditions have to be carefully optimized to minimize unwanted changes upon helicity reversal. Non-invasive measurements of beam parameters (charge Q, beam position $\mathrm{X}$ and $\mathrm{Y}$, beam direction $\mathrm{X}^{\prime}$ and $\mathrm{Y}^{\prime}$ and beam energy E) are made continuously during normal data taking. In this section, several important beam monitor devices used in Hall $\mathrm{C}$ for the Q-weak experiment will be introduced. A summary of helicity correlated beam properties for Q-weak Run I is given in Table 6.3.

\section{Beam Charge Monitors (BCM)}

Q-weak employs many of the standard Hall C instruments for beam diagnostics, including beam current monitors and beam position monitors. A linear, low noise beam charge monitor (BCM) is essential, in order to provide accurate normalization for the Q-weak asymmetry measurements, and to measure helicity-correlated beam current fluctuations which would lead to false asymmetries. 
Q-weak uses cavity-style BCMs to measure beam current. These are cylindrical "pillbox" RF cavities resonant in the transverse EM mode $\mathrm{TM}_{010}$ at the beam acceleration frequency of $1.497 \mathrm{GHz}$. When the electron beam excites this resonance, the associated power is temporarily stored inside the cavity. Part of this RF power can be read out by an antenna, providing an output signal proportional to the beam intensity.

The cavity BCMs possess long-term stability, good linearity and a large dynamic range. Their gains are periodically calibrated with respect to the Unser monitor [73], a parametric current transformer, which itself is self-cross-calibrated to a precise current source. One of the important requirements for the BCMs is that the noise in the beam charge measurement must be small with respect to the counting statistical error in Q-weak main detectors. During Q-weak commissioning and Run I, four BCMs located upstream of the target were operated. The Q-weak main detector yield was normalized to a combination of two adjacent monitors, BCM1 and BCM2. The noise contributions from BCMs ( $\sim 50 \mathrm{ppm}$ ) were estimated to be acceptable (see Section 6.2.4).

\section{Beam Position Monitor (BPM)}

A set of beam position monitors (BPMs) is used to measure the beam position and angle incident on the Q-weak target. Beam position is measured with stripline BPMs, which consist of four 1/4wavelength antenna wires $\left(X^{+}, X^{-}, Y^{+}, Y^{-}\right)$oriented 90 degrees to each other and parallel to the beam axis. At $1.497 \mathrm{GHz}$, beam power is coupled to the antennae, and the output signal amplitude on each wire depends on the distance from the beam.

Neglecting the finite length and radii of the antennae, as well as the finite size of the beam, to first order, the coupling of the beam to each antenna can be expressed as $V \propto$ constant $+r$, where $r$ is the distance of the beam from the BPM center [74]. Assuming, for example, that the beam is at $Y=0$, then $r$ is the coordinate $X$, and the beam position is given by

$$
X \propto \frac{V^{+}-V^{-}}{V^{+}+V^{-}}
$$


The BPMs are instrumented with Switched Electrode Electronics (SEE). The SEE is a sampleand-hold module; a single electronics chain is rapidly switched between the BPM antennae. Via this technique, electronic gain drifts are cancelled. Offset drifts and non-linearities in the electronics are approximately cancelled if the beam is centered at the BPM so that the $V^{+}$and $V^{-}$signals are of comparable magnitude. Therefore, the BPM has excellent long-term stability with the calibration set simply by geometry.

Instrumented with SEE, the BPMs are stable and linear over large current ranges, and have good signal to noise ratios with useful bandwidths $>10 \mathrm{kHz}$. The noise of the 4-wire SEE BPMs for beam currents above $10 \mu \mathrm{A}$ was shown to meet Q-weak beam requirements [75]. They also can be used to make relative beam energy measurements by using the known dispersion of the beam in the Hall $\mathrm{C}$ arc $(40 \mathrm{~mm} / \%)$ and measuring the position in a BPM in the arc. Absolute beam energy measurements are made in dedicated runs using the superharp [76] where the beam is arranged in a dispersive tune. In addition to the 4-wire BPMs, several sensitive cavity position monitors are also available for the beam position measurements. Q-weak uses many BPMs to extrapolate the beam position and angle at the target during Run I, for example, BPM3h04 located $18.9 \mathrm{~m}$ upstream and BPM3h09 3.9 m upstream of the Q-weak target were used to deduce the beam position at the target [77]. A slow position lock on the target is implemented by using feedback from these BPMs to adjust steering magnets, so that the position and angle remain within acceptable limits.

\section{Halo Monitors}

Halo monitors are installed on support frames referred to as the halo girder and the Q-weak girder (see Fig. 2.9). The main purpose of these detectors is to monitor beam halo, defined as any primary beam well outside the nominal beam envelope. Beam halo is mainly caused by beam scraping in apertures in the accelerator, or interactions with residual gas in the beamline. A $2 \mathrm{~mm}$ thick aluminum halo target is installed in the beamline on the halo girder. The halo target has both an 8 $\mathrm{mm}$ square, and a $13 \mathrm{~mm}$ diameter aperture (the one normally used) as a continuous halo monitor during production running. Electrons scattering from the targets can be detected by the various halo 
monitors located at different positions.

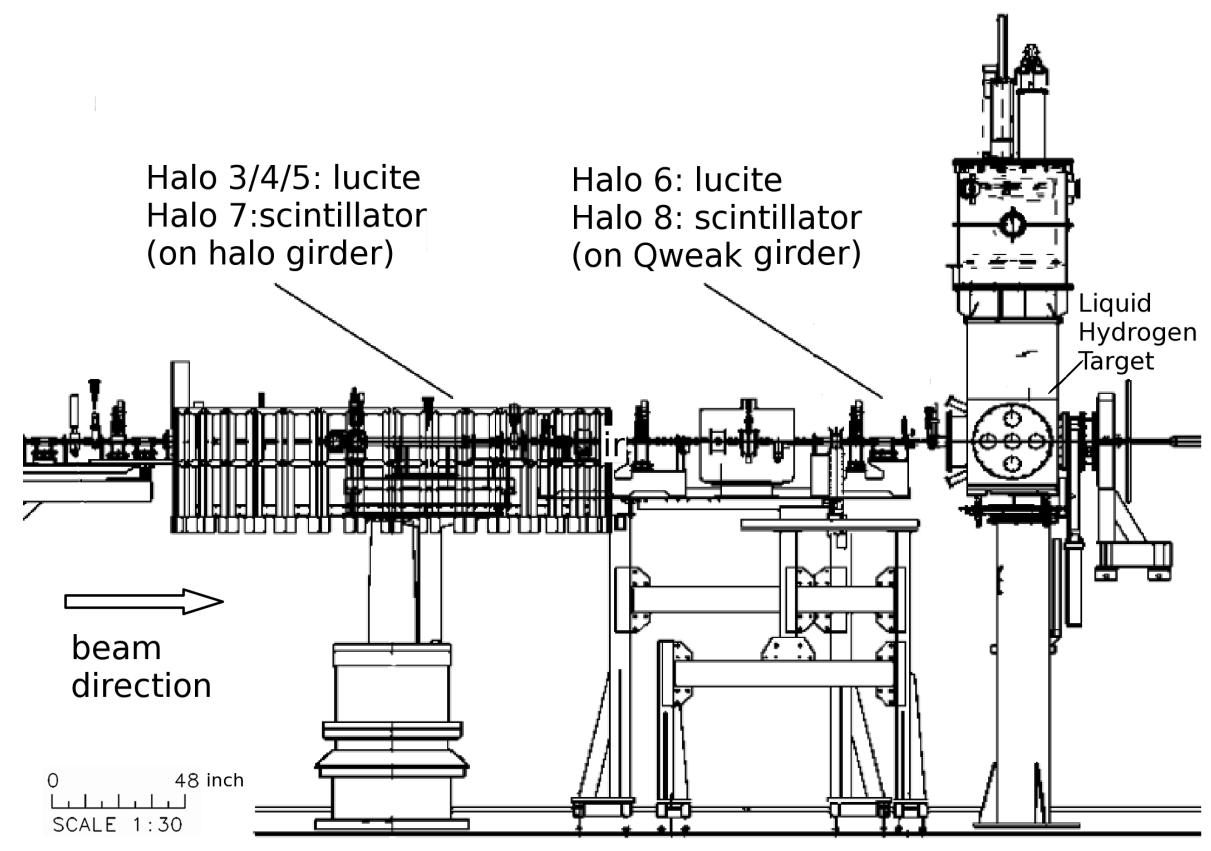

Figure 2.9: The position of the halo monitors for the Q-weak experiment [78].

Each halo monitor consists of a piece of lucite or scintillator coupled to a PMT; incident radiation generates photons in the lucite (scintillator) that are detected by the PMT. Two materials (lucite and scintillator) are used in halo monitors as they have different sensitivities to the incident neutral particles. Halo monitors 3, 4, 5 (with lucite) and 7 (with scintillator) are located on the halo girder, while halo monitors 6 (with lucite) and 8 (with scintillator) are located on the Q-weak girder, closer to the Q-weak target. During production running, the halo monitor rates are displayed in real time to assist in monitoring Qweak beam quality. A typical "good halo" rate for Q-weak Run I is about $10 \mathrm{~Hz} / \mu \mathrm{A}$ in halo 3 , within its tolerance of $20 \mathrm{~Hz} / \mu \mathrm{A}$; the rate limit for halo 4 is $33 \mathrm{~Hz} / \mu \mathrm{A}$. Beam conditions are considered to be bad once the beam halo rates go beyond these limits. 


\section{Beam Modulation}

In addition to minimizing helicity-correlated beam properties, it is also important to be able to apply corrections to the Q-weak asymmetry data to account for any residual via beam modulations. This requires us to know both the helicity-correlated differences in beam parameters, and the sensitivity of the Q-weak main detector to each beam parameter variation.

Detector sensitivities to beam property changes are measured using either natural beam motion, or beam modulation in dedicated studies. For the latter, four air-core magnetic dipoles in the Hall $\mathrm{C}$ beamline are used to purposely modulate the beam position and angle in $x$ and $y$. In addition, the beam energy $E$ is modulated with a Superconducting Radio Frequency (SRF) cavity. Based on these applied modulations, the corresponding detector sensitivities are determined. One advantage of this method is that it allows for good separation of position and angle changes, which are usually correlated in natural beam motion.

\section{Beam Raster}

The very small intrinsic size of the primary electron beam $(\sim 100 \mu \mathrm{m}$ diameter $)$ requires that it be scanned by a fast raster (FR) system over the $\mathrm{LH}_{2}$ target to avoid damage of target system due to beam heating effects. Beam is steered uniformly over a large area by using the FR system installed in the Hall C beam line tunnel, about $21 \mathrm{~m}$ upstream of the Q-weak target [79].

The FR system consists of two air-core magnets for independent steering in $x$ and $y$ at high frequency. During Q-weak full current runs, a $4 \mathrm{~mm} \times 4 \mathrm{~mm}$ square raster pattern was used. The FR pattern was generated by using two triangular steering signals with frequencies $f_{x}=24.96 \mathrm{kHz}$ and $f_{y}=25.08 \mathrm{kHz}$. The system generates a highly uniform beam intensity distribution on the target, and dramatically reduces beam heating effects. 


\subsection{Target}

The Q-weak experiment employs a $35 \mathrm{~cm}$ (4\% radiation length $\left.{ }^{2}\right)$ long cryogenic liquid hydrogen target, which was designed to be operated at $180 \mu \mathrm{A}$ with beam power of $2500 \mathrm{~W}$ [80]. The basic design goal was to provide high luminosity $\left(L=1.8 \times 10^{39} \mathrm{~cm}^{-2} \mathrm{~s}^{-1}\right)$ while minimizing density fluctuations which would increase the widths of the measured asymmetries.

A schematic view of the Q-weak target loop is presented in Fig. 2.10(a). The $\mathrm{LH}_{2}$ target is contained in an aluminum flask located at the bottom of the loop (see Fig. 2.10(b)). A cryogenic pump circulates $\mathrm{LH}_{2}$ in the loop and produces transverse flow relative to the beam direction. A heater and a heat exchanger in the loop are used to regulate the temperature of $\mathrm{LH}_{2}$ to the nominal $20 \mathrm{~K}$ at the design pressure of 35 psia without boiling or freezing. Thermometers and pressure gauges along the loop provide continuous monitoring of these crucial operating parameters.

Aluminum has a factor of ten larger parity-violation asymmetry than $\mathrm{LH}_{2}$ (with the opposite sign) at Q-weak kinematics. In order to minimize contributions from the aluminum target vessel to the measured asymmetry, the $\mathrm{LH}_{2}$ cell was designed in a cone shape with its wall and end-windows as thin as possible given the requirements for safe operation. The cell thickness is $0.254 \mathrm{~mm}$ for the wall, $0.127 \mathrm{~mm}$ for the entrance window and $0.127 \mathrm{~mm}$ for the central nipple of the $0.508 \mathrm{~mm}$ thick exit window. The cell windows are machined with a certain radius of curvature to reduce helicity-correlated changes in target length upon beam motion. With this design, the total rate from aluminum target windows at $180 \mu \mathrm{A}$ beam current is about $27 \mathrm{MHz}$ per octant, or $3 \%$ of the elastic rate from the $\mathrm{LH}_{2}$ itself.

The target heat exchanger obtains cooling power from both the $4 \mathrm{~K}$ Central Helium Liquefier (CHL) and the $15 \mathrm{~K}$ End Station Refrigerator (ESR) through two separate transfer lines. Heaters in the target are controlled via feedback loops that take information from one of the $\mathrm{BCMs}$ so that the target temperature is stabilized in response to changes in beam current.

Many studies were performed to characterize the effects due to boiling and density fluctuations

\footnotetext{
${ }^{2}$ Radiation length, usually denoted by $X_{0}$, is a characteristic length of matter, over which a high-energy electron loses all but $1 / e$ of its energy $E$ by bremsstrahlung, i.e. $-d E / d X=E / X_{0}$; and $X_{0}=7 / 9$ of the mean free path for pair production by a high-energy photon.
} 


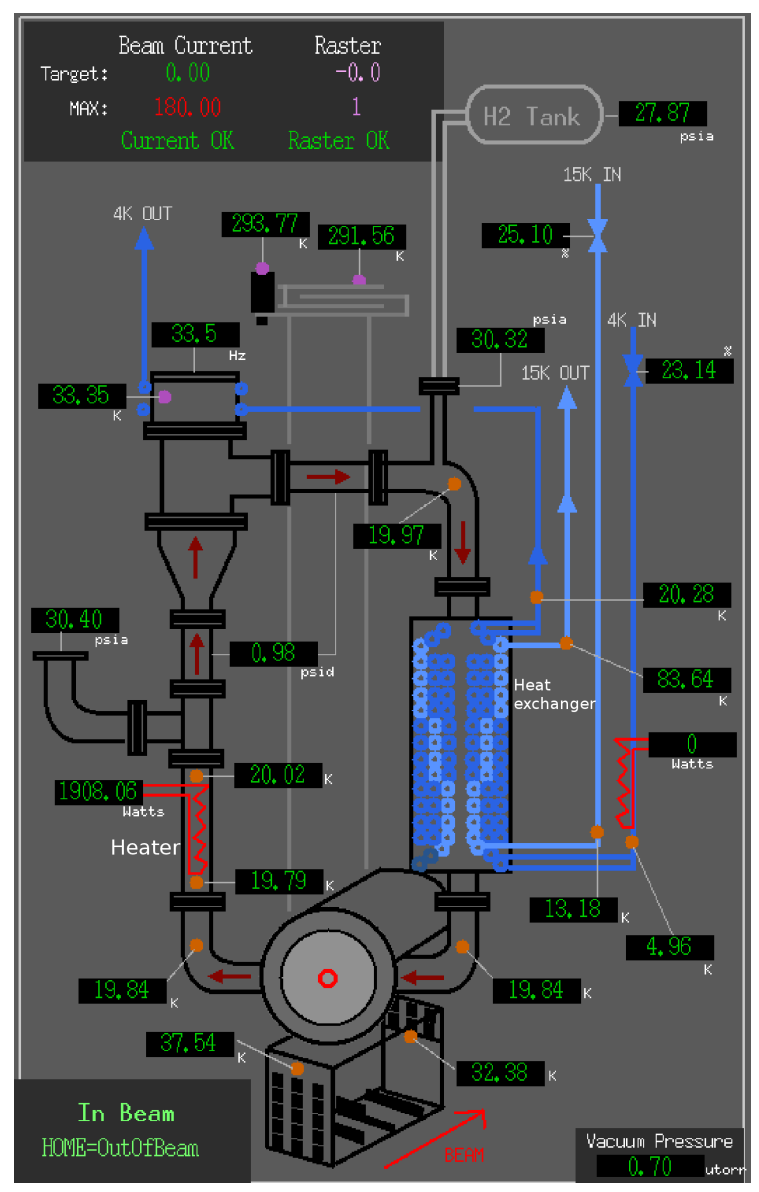

(a)

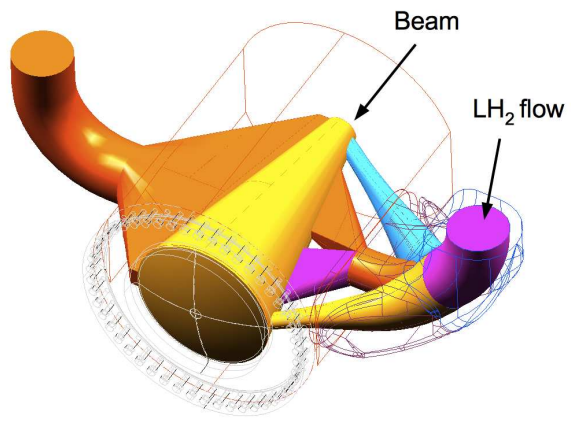

(b)

Figure 2.10: Two views of the Q-weak high power cryo $\mathrm{LH}_{2}$ target. (a) The target control GUI shows the schematic layout of the target and recirculating loop. (b) A zoomed-in view of the Qweak $\mathrm{LH}_{2}$ target cell [80].

with various beam current, pump speed, raster size and helicity reversal frequency settings. During the planning stages of Q-weak, tests with existing JLab targets indicated that the effects of target density noise could be greatly reduced (by a factor $\sim 10$ ) by running the experiment at an increased helicity reversal frequency. This necessitated changes to the helicity control system at the polarized source. The Q-weak experiment adopted $960 \mathrm{~Hz}$ as the nominal helicity reversal frequency, whereas previous experiments used $30 \mathrm{~Hz}$. During Q-weak commissioning, the boiling contribution to the main detector asymmetry width was measured to be $45 \mathrm{ppm}$ at $169 \mu \mathrm{A}$. The bulk density 
changes have been bounded to less than $0.2 \%$ at $150 \mu \mathrm{A}$. This performance meets the original design goals [81].

The Q-weak target also has 24 solid targets to allow for background and systematic studies. These targets are mounted in either the front or back panel of a box frame located beneath the $\mathrm{LH}_{2}$ cell so that the z-dependence of the backgrounds can be characterized. The targets include aluminum alloy dummy targets with different thicknesses for target cell window background studies, and carbon targets for $\mathrm{LH}_{2}$ boiling comparisons. A remote controlled motion system is used to select any given target.

\subsection{Spectrometer System}

The Q-weak spectrometer system is composed of an optics defining collimator system, a resistive toroidal magnet with 8-fold symmetry about the beam axis (QTOR), a set of 8 quartz Čerenkov detectors, and a set of tracking devices. A brief introduction will be given here with highlights on how the spectrometer achieves the requirements of this experiment.

\subsubsection{Collimator and Shielding Wall}

The Q-weak experiment utilizes a triple collimator system to select electrons scattered from the $\mathrm{LH}_{2}$ target. A picture of the system is displayed in Fig. 2.11. The middle collimator is the primary one, defining the acceptance for scattered electrons. The first and third collimators are mainly used to clean up Møller electrons and EM shower events. The collimators are made of lead alloy. The openings in each collimator are arranged in 8 azimuthally symmetric segments, corresponding to the 8 main detector octants. Extensive Monte Carlo studies were used to optimize the collimator design for maximum elastic rate at the main detectors with minimum contribution from inelastic events. The optimized openings define the azimuthal acceptance to $\pm 15^{\circ}$, and polar acceptance to $\left[4^{\circ}-13^{\circ}\right]$. The scattering angle acceptance defines the range of $Q^{2}$ for the measurement.

After selection by the collimator system, elastically scattered electrons are focused onto the 
main detector quartz bars by QTOR. Charged background particles are deflected out of the bar acceptance. Neutral backgrounds from the target that pass through the collimators are blocked by the shielding wall constructed downstream of QTOR.

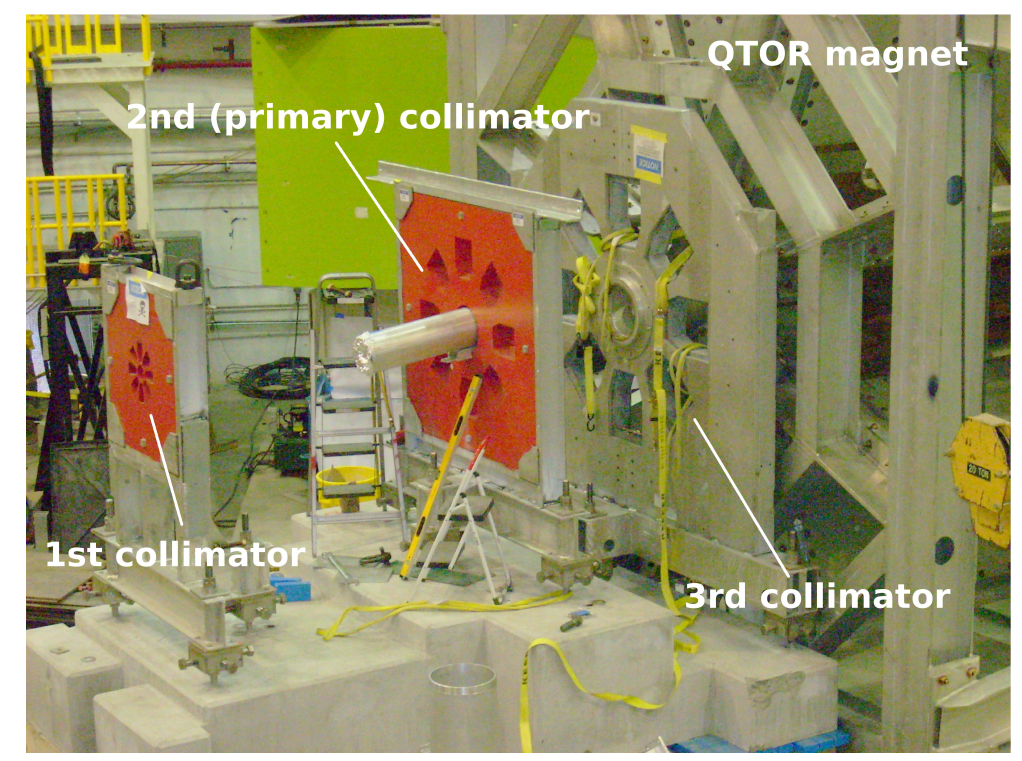

Figure 2.11: A view of the Q-weak collimator system during installation in the experimental hall [1]. The scattered electron beam entering from the left will go through the first (red) cleanup collimator, the middle (red) primary collimator and the third cleanup collimator into QTOR.

The shielding wall is a concrete wall with eight trapezoid-like apertures through which elastically scattered electrons are admitted. The apertures are designed to be large enough so that they do not affect the acceptance for elastic electrons. The wall also blocks photons and low energy electron backgrounds generated in upstream beamline.

\subsubsection{Toroidal Magnet}

A resistive toroidal magnet (QTOR) is used to focus elastically scattered electrons onto the Q-weak main detectors. QTOR has eight open sectors symmetrically located around the beam axis. Each sector is made up of two racetrack-shaped copper coils, with each layer consisting of two $2.20 \mathrm{~m}$ long straight sections, and two semicircular curved sections with inner radius $0.235 \mathrm{~m}$ and outer 
radius $0.75 \mathrm{~m}$. The copper conductor has a cross section of $5.84 \mathrm{~cm}$ by $3.81 \mathrm{~cm}$ with a center hole of $2 \mathrm{~cm}$ in diameter for water cooling [82]. The coils provide a field integral value of about $0.67 \mathrm{~T} \cdot \mathrm{m}$ along the central trajectory of elastically scattered electrons, leading to a nominal bend angle of $10^{\circ}$ for the electrons scattered at $\sim 8^{\circ}$.

The QTOR magnetic field was carefully calculated via numerical integration of the Biot-Savart law over the conductor's current distributions based on measured dimensions. The magnetic field was also mapped to verify these calculations. A photograph of QTOR during installation and field mapping is shown in Fig. 2.12. During Q-weak commissioning and Run I, QTOR was operated at the nominal 8921 A DC current.

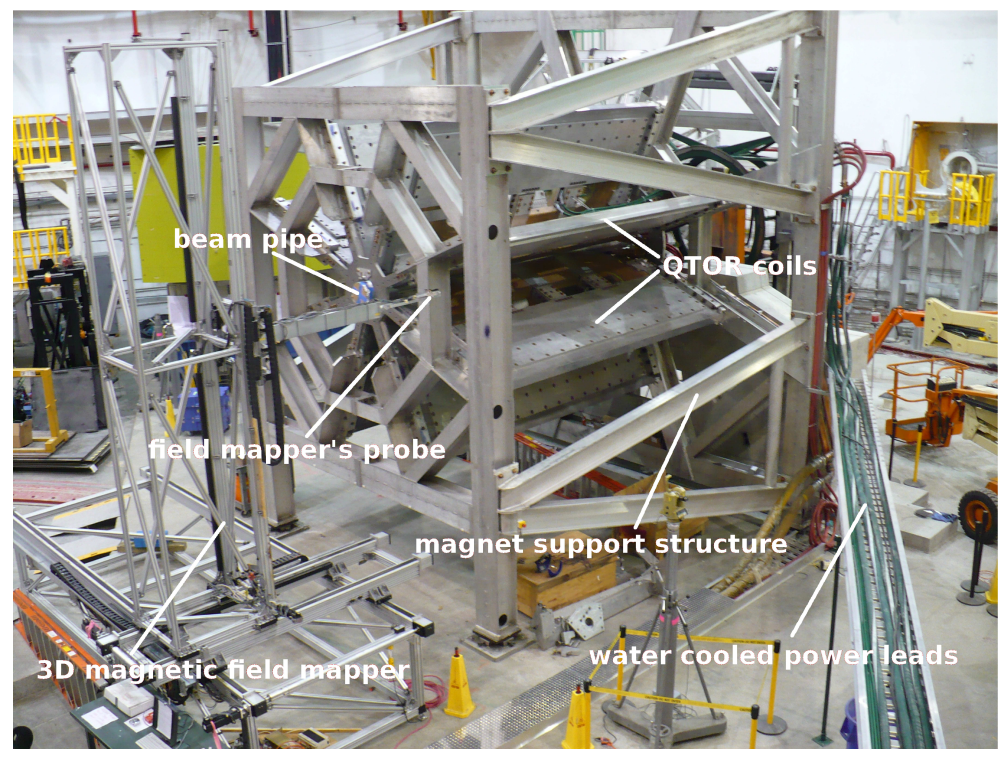

Figure 2.12: The QTOR magnet installed in Hall C [1]. A 3D magnetic field mapper was used to measure the magnetic field.

\subsubsection{Main Detectors}

Q-weak employs a set of eight quartz Čerenkov detectors to sense elastically scattered electrons, as Čerenkov detectors are insensitive to neutral backgrounds. The detector active elements are fused-silica quartz (Spectrosil 2000) bars which are radiation hard, arranged symmetrically about 
the beam axis in the focal plane of the spectrometer. Each $2 \mathrm{~m}$ long bar consists of two $1 \mathrm{~m} \times 18 \mathrm{~cm}$ $\times 1.25 \mathrm{~cm}$ bars glued together end-to-end (see Fig. 2.13(a)). The resultant quartz bar is fixed within an aluminum detector housing and shielded from external light with two covers. Čerenkov light generated by electrons travels along the bar via total internal reflection and is collected by 5 inch (12.7 cm diameter) PMTs attached to either end of the quartz bar ${ }^{3}$. The Čerenkov signals are read out either in current mode with low gain $(\sim 2000)$ bases for asymmetry measurements or in counting mode with high gain $\left(2 \times 10^{6}\right)$ bases for tracking measurements. The detectors are supported by aluminum exoskeletons and mounted on a Ferris wheel structure (see Fig. 2.13(b)).

To achieve the desired statistical precision within the allocated beam time, the Q-weak main asymmetry is measured at a high beam current of $150-180 \mu \mathrm{A}$. At full beam current, the rate of elastic electrons impinging on each bar is $800 \mathrm{MHz}$, giving the total rate of $6.4 \mathrm{GHz}$ for eight detectors. This rate is too high to employ pulse-counting techniques; therefore, the asymmetry data are acquired in current mode. The output DC current $(\sim 6 \mu \mathrm{A})$ from the low gain PMT bases is amplified by I-V converters with gain of $1 \mathrm{M} \Omega$, and digitized by 18-bit ADC's. The main detector electronics chain achieved noise well within acceptance criteria, being much smaller than the statistical width due to the photons from the Čerenkov process.

During tracking mode runs (discussed in Section 2.4.4), the beam current is reduced to $\lesssim 100 \mathrm{pA}$, and the main detectors are operated in pulse counting mode using high gain PMTs and countingmode electronics. The main detector light yield for electrons was initially characterized in tracking mode. It was found that one track generates $\sim 16$ photo-electrons in each main detector. To increase this light yield and hence the signal-to-noise $(\mathrm{S} / \mathrm{N})$ ratio, a $2 \mathrm{~cm}$ thick $\mathrm{Pb}$ pre-radiator was installed in front of each quartz bar. This increased the light yield by a factor of seven and improved S/N by $\sim 20$. However, shower fluctuations in a pre-radiator also introduce an excess noise $(\sim 10 \%)$ to the octant averaged asymmetry. Essentially all the phase I data were acquired with this configuration. A detailed discussion of the main detector design, construction, installation, tests and performance can be found in Ref. [83].

\footnotetext{
${ }^{3}$ The Čerenkov signal generation process is discussed in detail in the context of the Q-weak scanner detector in Section 3.4.
} 


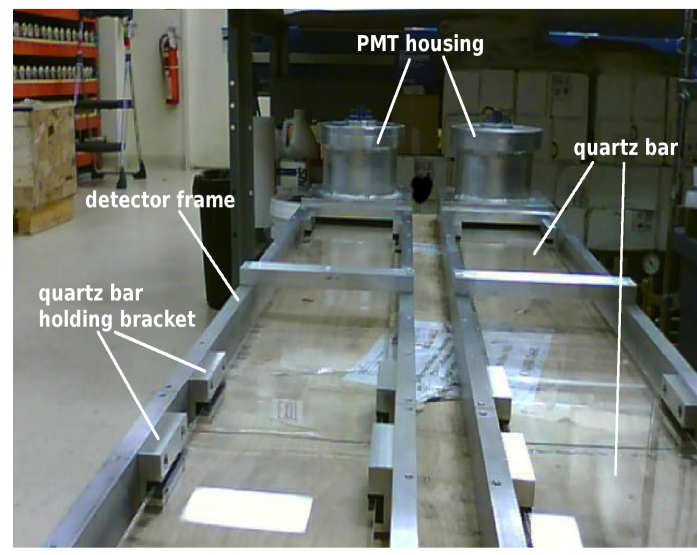

(a)

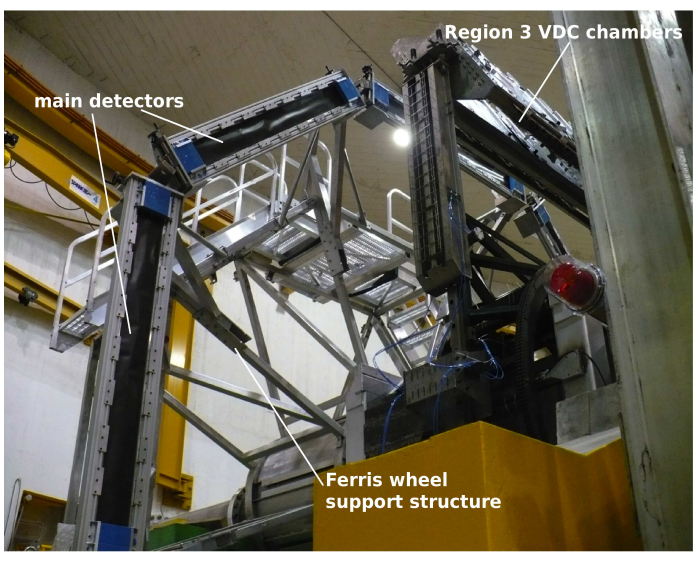

(b)

Figure 2.13: (a) Main detector assembly at JLab EEL building. (b) Main detector installed on the Ferris wheel support structure [1].

\subsubsection{Tracking System}

The Q-weak tracking system is designed to map the $Q^{2}$ acceptance of the experiment, to measure the light-weighted response of the main detectors, and to characterize backgrounds. For example, the goal of measuring the proton's weak charge to $4 \%$ requires that the average $Q^{2}$ weighted by the main detector Čerenkov light yield be determined to $1 \%$. The main detectors being operated in current-integrating mode necessitates weighting of the kinematic $Q^{2}$ by light yield. This is referred to as the light-weighted $\left\langle Q^{2}\right\rangle$.

A set of tracking detectors are operated in calibration runs at beam currents below $100 \mathrm{pA}$, so that tracks from individual scattered particles can be determined. Two sets of tracking detectors are mounted on rotatable frames so that they may be positioned in two opposing octants. Thus, the entire detector acceptance can be mapped in four sets of tracking measurements. The detectors are retracted radially outwards and parked outside the main detector acceptance when not in use.

\section{Region 1 GEMs}

The Region 1 gas electron multipliers (GEMs) were designed to make measurements at the location right after the first collimator, $1.07 \mathrm{~m}$ from the target. The GEMs would provide a single position 
point for a track, and together with Region 2 chambers, 13 plane points would be used to reconstruct the scattering vertex. The GEMs did not function during Q-weak Run I. Therefore they receive no further mention in this thesis.

\section{Region 2 HDCs}

The Region 2 horizontal drift chambers (HDCs) are located downstream of the primary collimator, $3.4 \mathrm{~m}$ from the target (see Fig. 2.2). Their purpose is to establish the initial trajectory of the scattered electrons before deflection in the QTOR magnet, and to track back to the target to find the interaction vertex [84].

In general, a drift chamber consists of planes of parallel sense wires at zero potential strung between cathode planes to which a negative high voltage is applied. The assembly is contained in a support structure with thin entrance and exit windows, and filled with a customized gas mixture (65\% argon and 35\% ethane during the tests at Virginia Tech; 50\% argon and 50\% ethane during Q-weak Run I). In each sense wire plane, field wires are placed between the sense wires to form drift cells by shaping the electric field. When a charged particle passes through a chamber and ionizes the gas in particular cells in each plane, the resulting electrons drift toward the sense wire along the electric field lines in the cell. The measured drift time between an external trigger signal and the resulting sense wire signal reflects the drift distance of the charged particle as it passed through the wire plane. However, the hit location can not be identified since the particle may go through that plane on either the left or the right side of the triggered wire. This phenomenon is referred to as the left-right ambiguity. By sequencing sets of planes with different wire orientations, left-right ambiguities can be removed, and the particle's trajectory through a multi-plane drift chamber can be determined.

In Region 2, each of two octants is covered by a pair of HDC chambers, which are separated by $42 \mathrm{~cm}$ along the beam direction. Each chamber consists of 6 wire planes $\left(x, u, v, x^{\prime}, u^{\prime}, v^{\prime}\right.$, representing wire orientations which are defined in Section. 4.2) with 32 sense wires per plane. The different wire orientations and wire offsets in those planes help to identify good tracks and remove left-right 


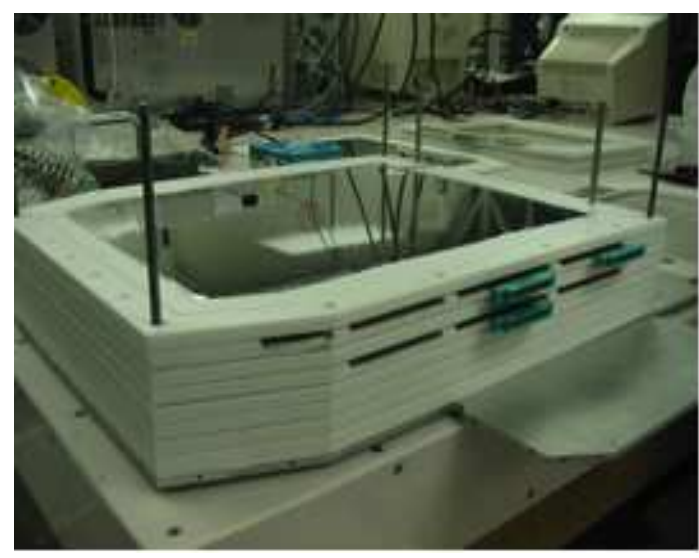

(a)

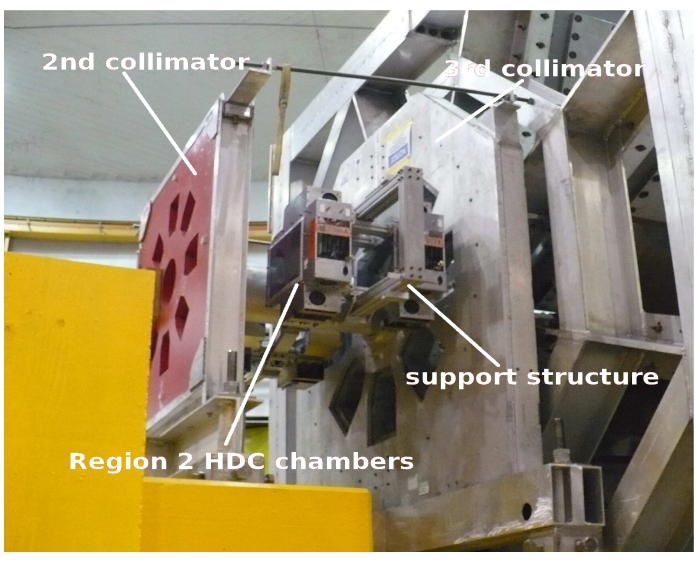

(b)

Figure 2.14: (a) The Region 2 HDC chambers constructed at Virginia Tech. (b) The HDC chambers in their motion mechanism installed between the second (primary) collimator and the third (cleanup) collimator $[1,85]$.

ambiguities. The HDCs analysis reconstructs hit positions first, then reconstructs a track by measuring the drift time between the wire signal and the particle trigger signal, and then converting this time to drift distance based on a parameterized drift velocity. Through on-board Nanometric preamplifier discriminator cards, the total of 768 sense wire signals for the HDCs are digitized by JLab "F1" TDCs. The position resolution measured with the HDCs is $150-200 \mu \mathrm{m}$ with single plane efficiencies better than 99\% [85]. During Q-weak Run I, the HDCs encountered some hardware issues. It was found that there was $\sim 7 \mathrm{~ns}$ missing at the beginning of their drift time distributions; the single wire efficiency was reduced to $93 \%$ due to this issue [86]. The Region 2 chambers can be operated up to a beam current of about $1 \mathrm{nA}$.

\section{Region 3 VDCs}

The Region 3 vertical drift chambers (VDCs) are located after the QTOR magnet and just before the main detectors. They are used to measure the tracks after the magnet, and in conjunction with the HDCs, to determine the momentum of elastically scattered electrons, hence the $Q^{2}$ distribution and main detector light-weighted $\left\langle Q^{2}\right\rangle$. 


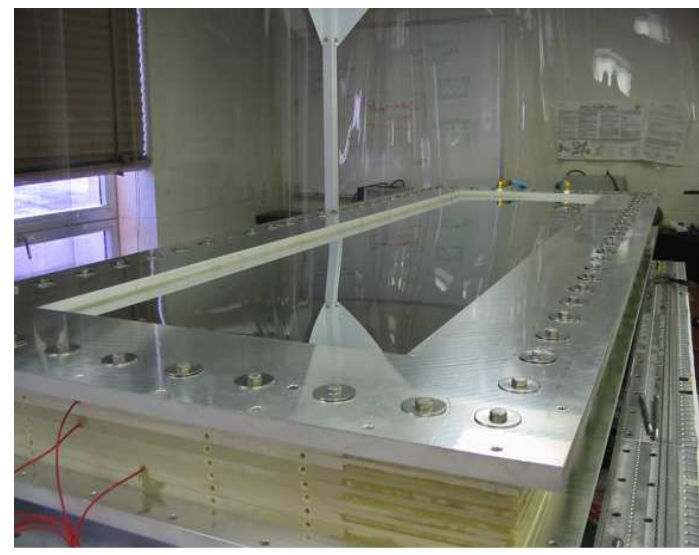

(a)

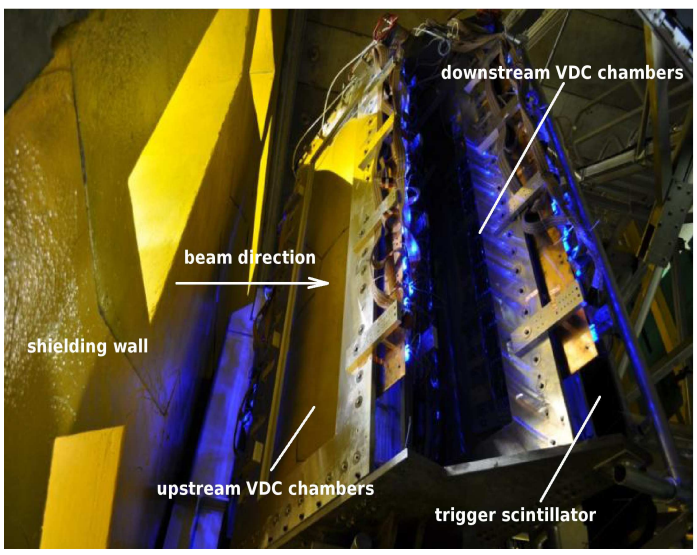

(b)

Figure 2.15: (a)The Region 3 VDC chambers constructed at The College of William and Mary. (b) The VDC chambers mounted on the Region 3 rotator right behind the shielding wall [1, 85].

In Region 3, there are a pair of chambers in each octant. Each chamber has an active area of $2.3 \mathrm{~m} \times 1 \mathrm{~m}$, and has 2 planes $(u, v)$ each with 281 sense wires. The total of 2248 wire signals are read out via multiplexing: one TDC channel shares 9 wires through delay lines, requiring only four 64-channel TDC modules. The resolution for an individual plane is $220 \mu \mathrm{m}$, with single plane efficiency better than $99.8 \%$ [85]. The VDCs are operable up to a beam current of about $100 \mathrm{nA}$.

Although both the HDCs and VDCs are drift chambers with similar working principles, there are some design differences between them in order to meet the specific Q-weak tracking requirements. Details about their differences and how to reconstruct tracks with them will be discussed in Chapter 4.

\section{Trigger Scintillator}

Two $220 \mathrm{~cm} \times 30.48 \mathrm{~cm} \times 1 \mathrm{~cm}$ trigger scintillators are used to provide a trigger and timing reference for the tracking system [87]. They are mounted on the Region 3 rotator behind the VDCs. As the picture shown in Fig. 2.16 illustrates, scintillation light is collected by PMTs attached to either end of the scintillator via light guides. The trigger time is obtained by averaging the time of the left and right PMT pulses by using a mean-timer. The timing resolution for these trigger scintillators is 


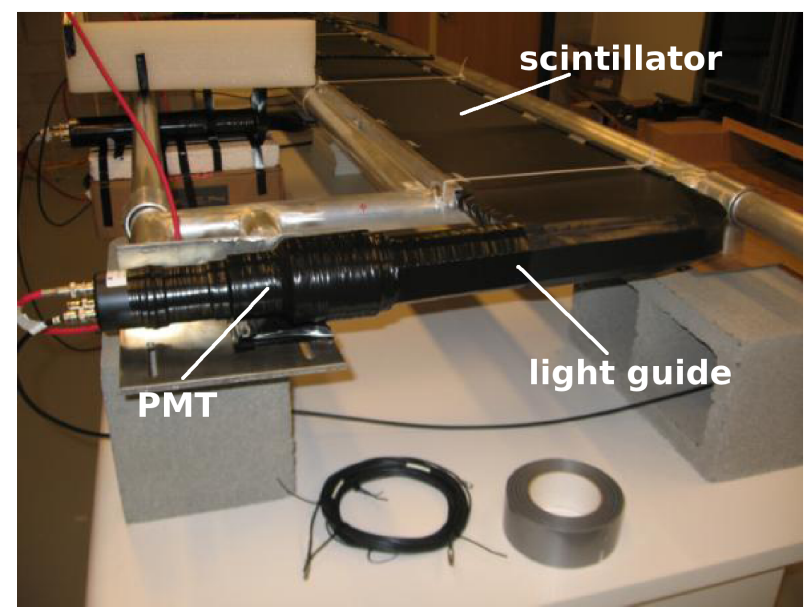

Figure 2.16: A view of one trigger scintillator on the bench [1, 87].

better than $460 \mathrm{ps}$, which is more than sufficient to provide a trigger signal and timing reference for the tracking chambers.

\section{Focal Plane Scanner}

The focal plane scanner was developed at the University of Winnipeg. This instrument was my main hardware responsibility for Q-weak; therefore, it will be the focus of a separate chapter (Chapter 3) of this thesis.

Tracking measurements are conducted at beam currents below $100 \mathrm{pA}$, about 3 orders of magnitude lower in current than the $A_{P V}$ measurement. In order to match the tracking results to high beam current, an extrapolation method is necessary. The focal plane scanner is designed for this purpose. It is also useful in monitoring spectrometer optics and for background studies.

The focal plane scanner is a small Čerenkov scanning detector with $1 \mathrm{~cm} \times 1 \mathrm{~cm}$ active area. It can be mounted either in front of or immediately behind the bottom octant of the main detector to sense the electron flux distribution in the focal plane. It was designed to be used in both tracking mode and production mode to extrapolate tracking results from low to high beam current. 


\subsubsection{Luminosity Monitors}

The Q-weak experiment employs two sets of luminosity monitors (LUMIs): the upstream and downstream LUMIs, with different functionalities. Both of them serve as sensitive diagnostic detectors.

The Upstream LUMIs The upstream LUMIs are an array of 4 small Cerenkov detectors situated on the upstream face of the primary collimator (see Fig. 2.17 (a)). They are $25 \mathrm{~cm} \times 7 \mathrm{~cm} \times 2 \mathrm{~cm}$ quartz bars coupled with air-core light guides to PMTs at both ends. They sense mainly Møller electrons scattered at $5^{\circ}$ and are used as target density fluctuation monitors. These detectors sense a total rate of $133 \mathrm{GHz}$, and therefore can provide precise measurements in a short time. Being symmetrically positioned around the beam line, they have high sensitivity to beam position, and thus can serve as a sensitive beam position monitor.

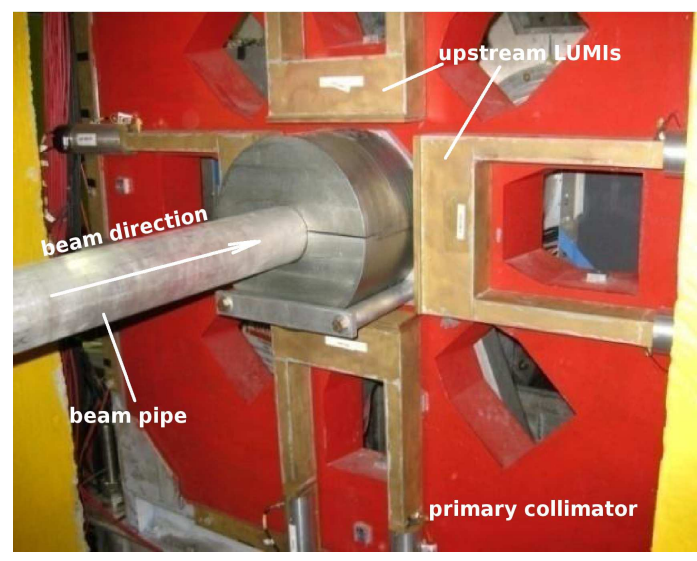

(a)

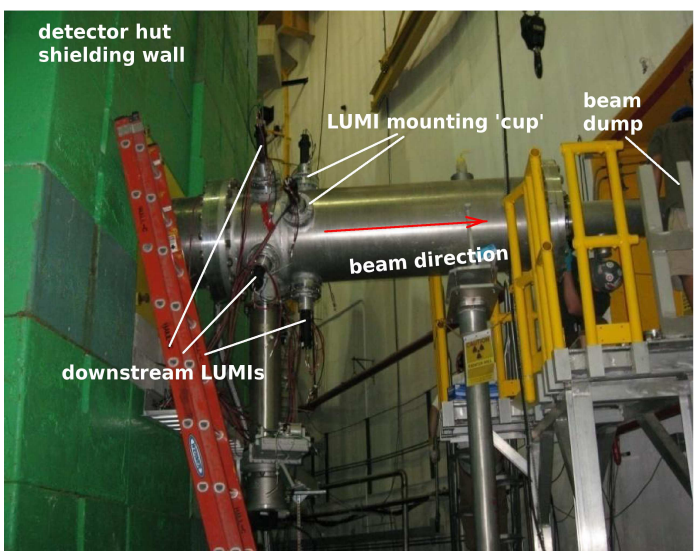

(b)

Figure 2.17: A view of the upstream and downstream luminosity monitors [1, 85]. (a) The 4 upstream LUMIs mounted on the upstream face of the primary collimator. (b) The 8 downstream LUMIs installed in the beam pipe near the end of Hall $\mathrm{C}$ beamline.

The Downstream LUMIs The downstream LUMIs, consisting of eight detectors, are designed to be null asymmetry monitors. Each LUMI is made up of a piece of $4 \mathrm{~cm} \times 3 \mathrm{~cm} \times 1.3 \mathrm{~cm}$ Spectrosil 2000 quartz positioned at $\sim 0.5^{\circ}$ from the beam axis. To reach this very small angle, the LUMI assemblies are inserted into special cups which penetrate deep into the beampipe far 
downstream of the target (see Fig. 2.17(b)). These detectors are each read out with a Hamamatsu 2-inch (5.08 cm diameter) quartz window PMT via a $35 \mathrm{~cm}$ long air light guide. The downstream LUMIs sense a total rate of $100 \mathrm{GHz}$ of scattered e-p elastic and Møller electrons (the rate ratio $\sim 1: 1$, giving very small statistical error. Since they are at very forward angles, both $Q^{2}$ and the parity violating asymmetry should be essentially zero (see Eqn. 1.49). These monitors are therefore very sensitive to false asymmetries arising from helicity-correlated beam properties, and can give immediate feedback for beam diagnostics [88]. They are designed to be operated in both event mode for tracking runs as well as current mode for parity runs. In event mode, the PMTs use high gain bases and $\times 10$ fast pre-amplifiers, read out by scalers or QDCs, while in integrating mode, unity gain bases are used and the PMT signals are amplified by $4 \mathrm{M} \Omega$ current to voltage pre-amplifiers and integrated by 18-bit ADCs.

\subsection{Data Acquisition}

The Q-weak Data Acquisition system (DAQ) [89] is built upon the CODA framework [90] developed at JLab. The system contains electronics crates, readout controllers (ROCs), trigger-supervisor (TS) module and DAQ computers, which run Linux and CODA systems. A schematic diagram of the DAQ system is shown in Fig. 2.18. The triggering and event control are performed by the TS, which is linked to each subsystem (or crate) via trigger interface modules. The TS serves as an interface between experiment-specific triggering and the DAQ system, and there are several different trigger sources (as listed in Table 2.3) available for it. Data from all crates are read out and transported back to the DAQ computer through ethernet and are saved in standard CODA format.

The Q-weak experiment has two modes of data acquisition: parity (current) mode and tracking (event) mode. The electronics for the different modes are arranged in different crates. ROC0 is in the crate containing the trigger-supervisor. ROC1 and 2 in the electronics room are used for parity mode, containing the 18-bit ADCs for main detectors and beam line monitors. ROC9, 10 and 11 in the electronics cage in the experimental hall are used for tracking mode, containing the TDCs, 
scalers for the GEM, HDC and VDC tracking detectors. ROC4 in the electronics room is also for tracking mode, corresponding to the VME (Versa Module Eurocard, a computer bus standard, widely used for many applications in the experimental subatomic field) crates housing the QDCs, TDCs, and scaler modules for the main detectors, trigger scintillators, and the focal plane scanner.

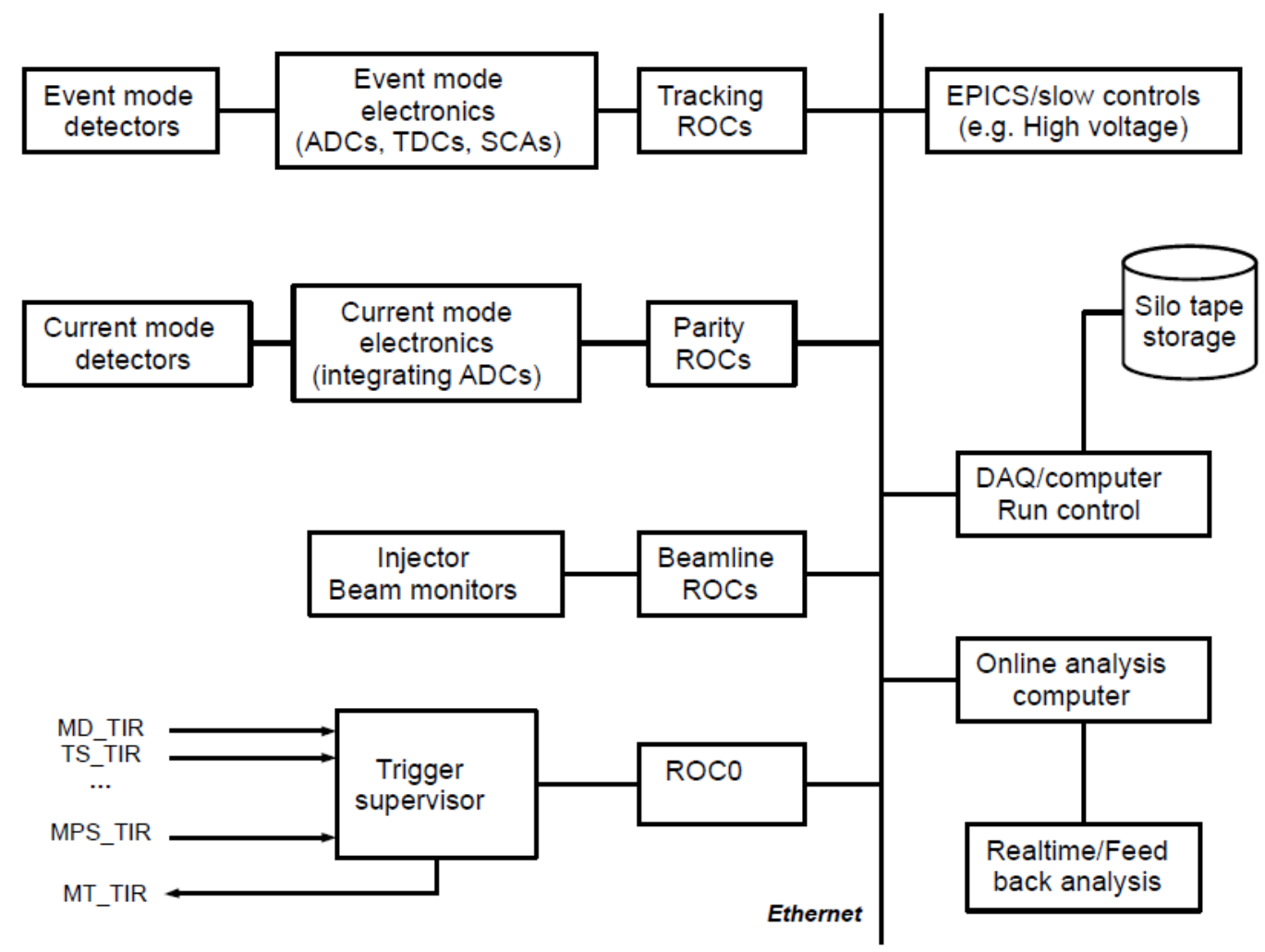

Figure 2.18: Schematic diagram of the Q-weak data acquisition system.

The main measurements of Q-weak use the parity mode DAQ to record main detector signals. The 16 channel output PMT currents from the main detectors are amplified by low noise trans-impedance pre-amplifiers (I-to-V converters) then read out by the TRIUMF 18-bit integrating ADCs [91]. Luminosity detectors and beamline instrumentation are read out with the TRIUMF ADCs as well. The Q-weak parity mode DAQ uses the MPS trigger to record at $960 \mathrm{~Hz}$ helicity reversal rate to measure the average voltage levels produced by the detectors and beamline instru- 


\begin{tabular}{l|ll}
\hline \hline Trigger Source & Type & Mode \\
\hline Region 3 trigger scintillator & TS_TIR & event mode \\
main detector & MD_TIR & event mode \\
quartz scanner & QS_TIR & event mode \\
Region 2 scintillator & R2_TIR & event mode \\
\hline helicity interval & MPS_TIR & event/current mode \\
Clock/pulser & Fake_MPS_TIR & event/current mode \\
\hline \hline
\end{tabular}

Table 2.3: Main trigger sources and types in Q-weak. There are also other trigger sources available, such as triggers from Region 1 detector, background detectors and random triggers. These triggers can be combined logically and pre-scaled to an appropriate rate by the trigger supervisor, to form the master trigger (MT_TIR) for the DAQ system.

mentation in each helicity interval. The resulting data flow is about $4.5 \mathrm{MB} / \mathrm{s}$, independent of beam current.

The tracking mode DAQ records a large number of channels from all elements of the tracking system for every event, which is triggered by the trigger scintillator. The HDC and VDC signals are read out by F1 TDCs and scalers. The event hits on main detector, trigger scintillator and scanner are recorded with VMEbus CAEN [92] V792 QDCs, JLab F1 TDCs and SIS3801 scalers. The tracking mode DAQ is able to read out all the channels at event rates up to $6 \mathrm{kHz}$.

\subsection{Data Analyzer}

The general Q-weak data analysis paths are depicted in Fig. 2.19. The data are analyzed by two analyzer codes: "QwParity" for parity mode data analysis, and "QwTracking" for event mode data analysis. Both analyzers have a data decoding stage, in which CODA files or datastream are decoded into physical data for each device according to its channel map, which contains the information on ROC number, data bank and channel number. In further analysis, the parity analyzer calculates the helicity quartet asymmetries, yields and the corresponding running averages, based on delay- 
reported pseudo-random helicity states. The tracking analyzer finds the partial tracks in HDCs and VDCs separately, then matches them to tracks and determines the momentum and momentum transfer for each reconstructed track.

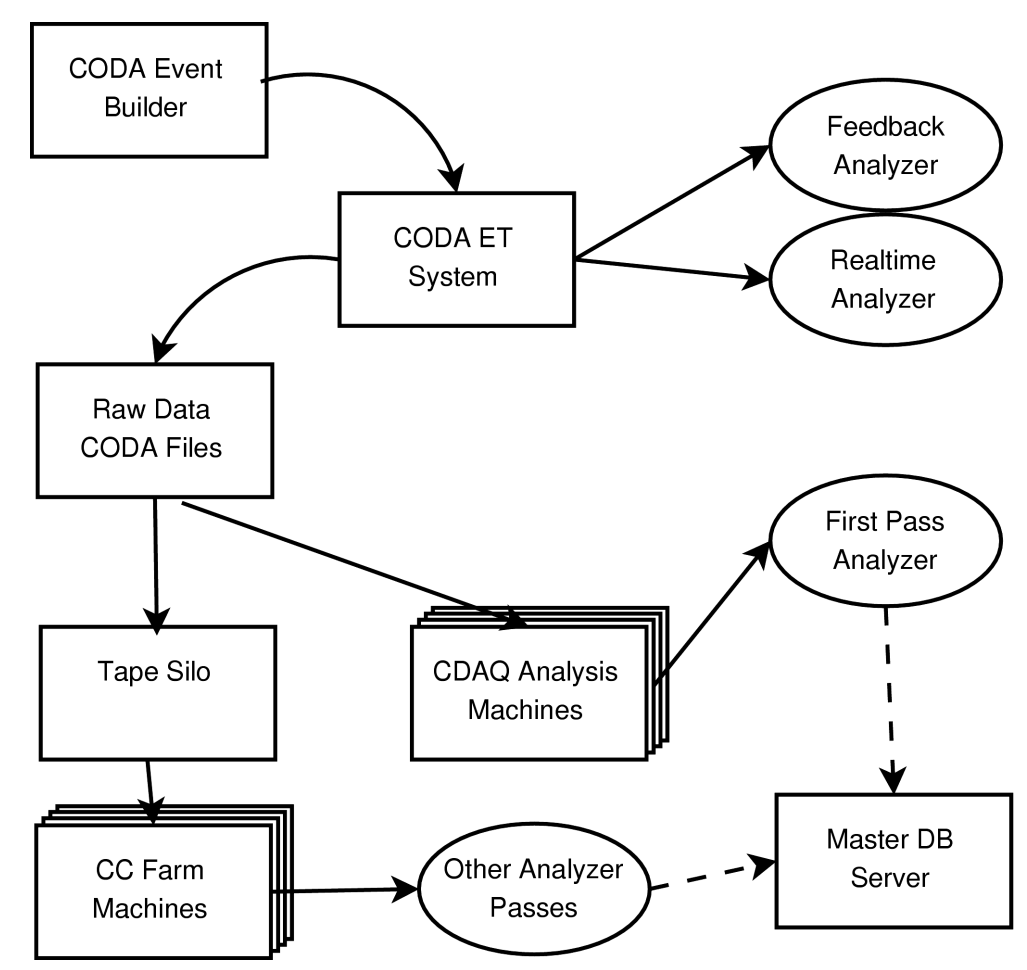

Figure 2.19: Schematic diagram of the Q-weak data analysis paths [93]. 


\section{Chapter 3}

\section{Electron Flux Profile Scanner}

The Q-weak electron flux profile scanner is a scanning Čerenkov detector designed to measure the rate distribution of scattered electrons in the focal plane of the spectrometer; it is thus also referred to as the Focal Plane scanner. A key criterion for the scanner design is the uniformity of its response with position as it moves across the acceptance of the Q-weak main detectors. This will be discussed in Section 3.8. A Čerenkov detector is used since it is insensitive to neutral particle backgrounds. The scanner system may be positioned immediately upstream or downstream of the main detector in octant 7 (see Section 4.2, the Q-weak coordinates and naming convention, for reference) to perform 2-dimensional scans of rates in a vertical plane over the fiducial area of that Čerenkov bar. The scanner possesses the unique ability to work in counting mode at both low and high beam current, and is the only detector in the experiment capable of making measurements of tracking parameters at high current. In this chapter, I present a chronologically ordered description of the evolution of the scanner's design leading up to its first commissioning results.

\subsection{Motivation}

As described in Chapter 2, in Q-weak, the $\left\langle Q^{2}\right\rangle$ determination and background studies are performed up to $100 \mathrm{nA}$ with the tracking system. The $A_{P V}$ measurement, on the other hand, is done at 
$150-180 \mu \mathrm{A}$, where the tracking detectors are inoperable. To obtain confidence in applying the results from tracking mode to parity mode, tracking results determined at low beam current must be extrapolated to high beam current over 3 orders of magnitude.

The SLAC E158 experiment [20], which measured the electron's weak charge (refer to the discussion in Chapter 1), successfully employed a similar scanning detector to map the spatial distribution of the scattered electron beam intensity [94]. That device was found to be crucial to the success of the experiment, since it was the only means with which to study the spectrometer optics and perform background studies. A similar scanning detector was also used in the HAPPEx e-p parity violation experiment $[95,36]$ at JLab. The Q-weak scanner design was based on the E158 scanner; therefore, the E158 scanner will be discussed in some detail here.

\subsection{E158 Scanner Review}

As shown in Fig. 3.1, the E158 scanner system consisted of 4 Čerenkov scanner detectors, which performed radial and azimuthal scans over both e-e and e-p scattering regions [94]. In E158, this system was used to determine optics parameters and confirm Monte Carlo predictions of rates (see Fig. 3.2) for high-energy ( $45 \mathrm{GeV})$, high-flux $\left(\sim 10^{11}\right.$ electrons $\left./ \mathrm{s} / \mathrm{cm}^{2}\right)$ scattered electrons.

As illustrated in Fig. 3.3, the active element of each E158 scanner detector was a piece of rectangular fused silica (synthetic quartz) of size $5 \times 5 \times 20 \mathrm{~mm}^{3}$, oriented at an angle of 45 degrees with respect to the incident beam direction. In order to increase the sensitivity of the device, a rhombic tungsten pre-radiator with edge dimensions of $5 \times 15 \times 15 \mathrm{~mm}^{3}$ was mounted on the upstream face of the quartz radiator. Čerenkov light generated within the quartz volume was transported out of the quartz, and reflected along a $19 \mathrm{~mm}$ diameter air-core light guide until being detected by a PMT, which was operated in integrating mode. 


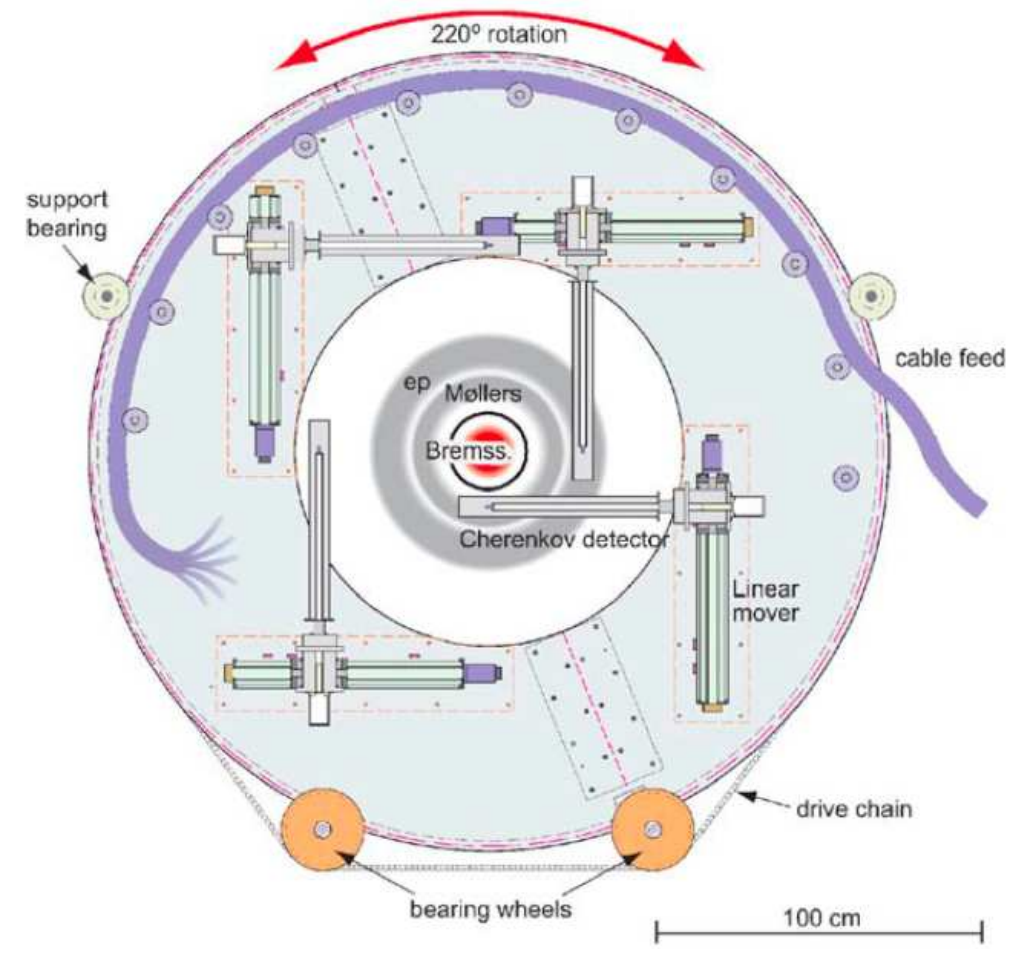

Figure 3.1: Beam's eye view of the E158 scanner system [94].

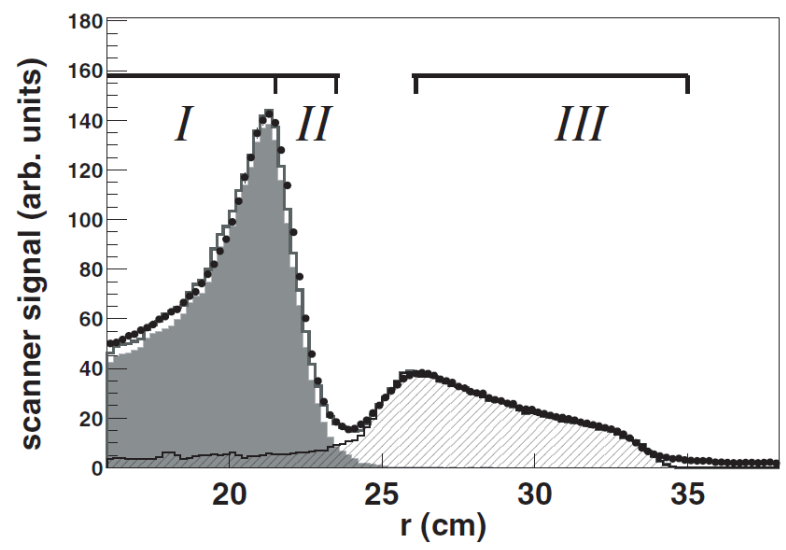

Figure 3.2: The radial rate distribution acquired by E158 scanner detectors [96], which validated the Monte Carlo results and hence expected physics backgrounds in E158. The points are scanner data, and the histogram is the Monte Carlo simulation. The shaded region is from the Møller scattering contribution; the hatched region is from the $e p$ scattering contribution. Møller and $e p$ asymmetries were measured by the PMTs in region I, III of the E158 calorimeter. 


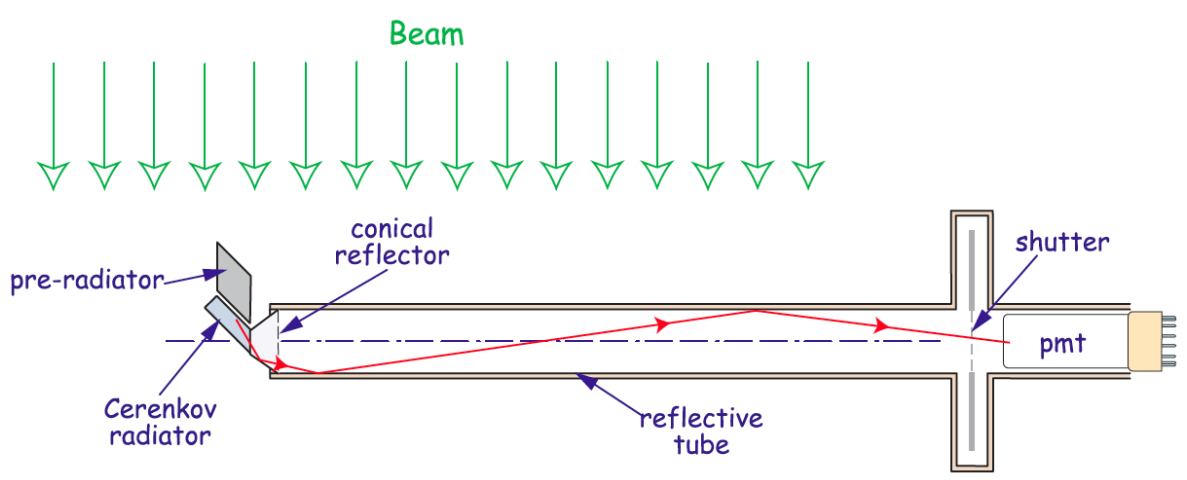

Figure 3.3: The schematic layout of an E158 scanner detector [94].

\subsection{Tailoring the E158 Scanner Design for Q-weak}

Taking the E158 scanner as a reference, a brief review of key design features and their optimization for Q-weak is presented here.

Use of quartz radiators Both E158 and Q-weak scanners use synthetic quartz as Čerenkov radiators; the type of quartz in the Q-weak scanner is the same as that used in the Q-weak main detectors. Quartz is insensitive to neutral backgrounds from gamma rays and neutrons, and is radiation hard, unlike scintillator. However, the design of Čerenkov light transport is more challenging than for scintillation light, especially for a device used in counting mode, as discussed below.

Counting mode versus current mode The E158 scanner was operated in current mode due to the high incident electron flux $\left(\sim 10^{5} \mathrm{MHz} / \mathrm{cm}^{2}\right)$. In contrast, for Q-weak, the maximum flux at the focal plane is $1 \mathrm{MHz} / \mathrm{cm}^{2}$ in parity mode with full beam current $(180 \mu \mathrm{A})$. This makes it possible for the scanner to operate in counting mode. In tracking mode runs, the rate is $<560 \mathrm{~Hz} / \mathrm{cm}^{2}$ below $100 \mathrm{nA}$ beam current. Based on these considerations, the Q-weak scanner was designed to operate in pulse counting mode over a large dynamic range of beam intensities. The use of counting mode also makes the scanner less sensitive to backgrounds than an integrating device. 
Light pipe diameter The E158 scanners used air-core pipe as light guides to reduce backgrounds arising from interactions with the light-guide material as opposed to the quartz radiator. The Qweak scanner design inherited this advantage of using air-core pipe, but based on the choice of a counting mode device, required better light transport for high efficiency operation. Light-tube associated backgrounds had been studied extensively by E158 aimed at improving the scanner's signal-to-background (S/B) ratio. It was found in those studies that a smaller diameter light guide led to a higher S/B ratio, but smaller signal [94]. The E158 scanner adopted a $19 \mathrm{~mm}$ diameter light guide to pursue a relatively higher $\mathrm{S} / \mathrm{B}$ ratio. The Q-weak scanner, in contrast, uses a larger $(51 \mathrm{~mm})$ diameter light guide.

PMT readout coincidence requirements In order to suppress backgrounds, the Q-weak scanner employs two light guides and PMTs viewing two separate pieces of quartz that are optically isolated from one another, operated in coincidence mode. Thus, better performance for both signal amplitude and $\mathrm{S} / \mathrm{B}$ ratio are achieved.

Cartesian versus polar scanning The E158 scanners measured circular electron-flux bands formed by the scattered electrons in e-e and e-p scattering, by performing "polar" scans in radial and azimuthal directions. Each scanner was mounted on a linear motion mechanism to scan radially relative to the beam axis, and a rotation annulus centered on the beam axis (see Fig. 3.1) to allow measurement of the entire profile of the scattered electrons. In contrast, the Q-weak scanner detector performs 2D Cartesian (the $x-y$ plane) scans along the bottom octant of the main detector (see Fig. 3.6). A full bar scan of the Q-weak scanner can cover a large area $(200 \mathrm{~cm} \times 26 \mathrm{~cm})$, in excess of the main detector dimensions, which allows it also to be useful for background studies.

Radiator tilt angle In the E158 scanner, the quartz radiator was constructed with a tilt angle of $45^{\circ}$ relative to the approximately parallel incident electron beam in order to increase light yield. For Q-weak, this was not necessary, since the electron beam is incident on the scan plane with an average angle of about $20^{\circ}$ in $\theta$ and azimuthal angle $\phi$ within range of $-25^{\circ}$ to $+25^{\circ}$; this allowed 
for a more compact layout (see Fig. 3.6).

Geometrical Considerations The Q-weak scanner has to fit in a relatively constrained region of space inside the main detector shielding hut. It has to be positioned as closely as practically possible to the Q-weak main detector of interest. The scanner detector was designed to allow its PMTs to stay outside the scattered electron beam envelope, minimizing radiation damage and direct backgrounds. Unfortunately, this led to location of the PMTs in a region of unexpectedly high gamma backgrounds, which posed a particular challenge (see section 5.1.1).

\subsection{Basic Q-weak Scanner Design}

\subsubsection{Detector}

The Q-weak scanner system consists of two main parts: a detector and a 2D linear motion assembly. The detector was designed to work in coincidence mode with two sensitive components and two light guides. The structure of the detector is depicted schematically in Fig. 3.4. Two pieces of fused silica (synthetic quartz) are used as Čerenkov radiators. They are optically decoupled from one another, and are each optically coupled to separate light guides arranged in a "V"-configuration. The air-core light pipes are lined with a highly reflective coating to improve light transport efficiency. The PMTs at the ends of the pipes are operated in counting mode.

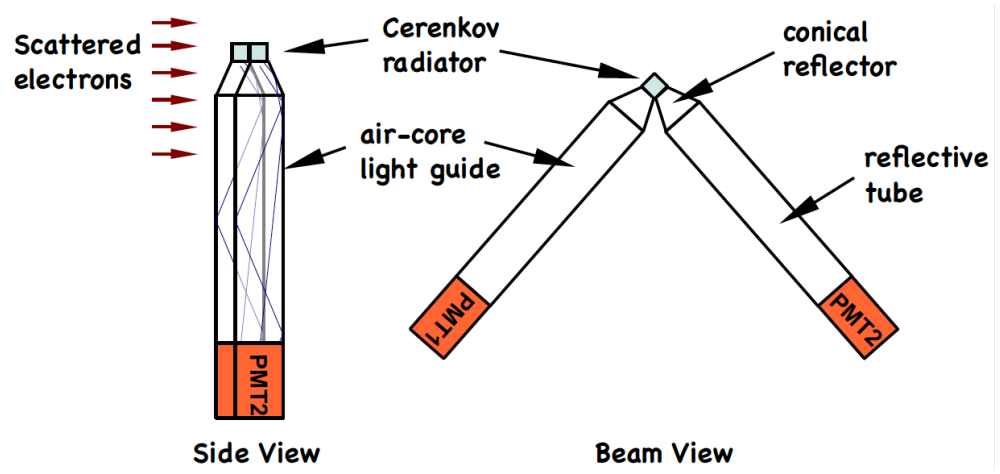

Figure 3.4: Schematic of the Q-weak scanner detector. 
The light generation process from which the scanner detector derives its signals is based on the Čerenkov effect. For a high-energy electron passing through a quartz element, faster than the speed of light in quartz, Čerenkov photons are generated in a cone-shaped distribution about the electron trajectory with an angle [9]:

$$
\cos \theta_{c}=\frac{1}{\beta n(\lambda)}
$$

where $n(\lambda)$ is the index of refraction of quartz for the photons with wavelength $\lambda ; \beta=v / c, v$ is velocity of the electrons, and $c$ is the speed of light in vacuum. The quartz radiators have $n(\lambda) \sim 1.47$ for photons with wavelength $\lambda \sim 360 \mathrm{~nm}$. For highly relativistic electrons, $\beta \sim 1$, and thus the Čerenkov cone angle $\theta_{c}$ in quartz is approximately $47^{\circ}$. Only incident electrons with an energy above $\sim 0.7 \mathrm{MeV}$ can emit Čerenkov photons and be detected. The number of photons produced per unit path length of an electron and per unit wavelength interval of the photons is given by $[9,97]$ :

$$
\frac{d^{2} N}{d \lambda d x}=\frac{2 \pi \alpha}{\lambda^{2}}\left(1-\frac{1}{\beta^{2} n^{2}(\lambda)}\right)
$$

From this formula, the number of Čerenkov photons generated inside the scanner radiators can be predicted. The Čerenkov light spectrum increases into the UV region at wavelengths below $400 \mathrm{~nm}$ (see Fig. 3.5). For a $1 \mathrm{~cm}$ thick quartz radiator, about 200 photons within wavelengths from $300-450 \mathrm{~nm}$ would be generated by each incident electron. Not all of these photons can be detected by PMTs due to light transport loss and the quantum efficiency of the PMT's photocathode (see Section 3.9, and Fig. 3.21 for the PMT wavelength response). Optimization of the light transport process is thus another important part of the detector design.

The two quartz elements are $1 \times 1 \times 1 \mathrm{~cm}^{3}$ cubes, positioned one in front of the other and as close as possible in the $z$-direction. Each cube is parallel to the vertical focal plane of the Q-weak spectrometer (the $x-y$ plane), and with no tilt relative to its light guide axis. The geometrical overlap of the two sensitive elements forms the fiducial area of the scanner detector, which is small enough to allow operation in counting mode. This size is also consistent with the typical spatial size of the variations of the scattered electron rate distribution in the focal plane. 


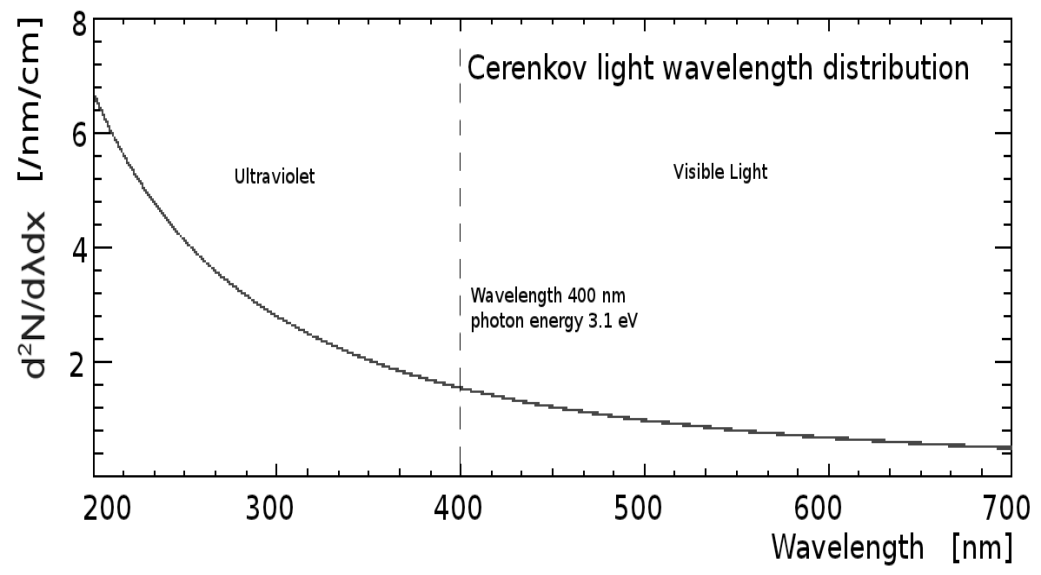

Figure 3.5: Number of Čerenkov photons generated by the incident electrons per unit wavelength $(1 \mathrm{~nm})$ and unit path length $(1 \mathrm{~cm})$ in quartz as a function of the photon's wavelength [98].

The detector light yield depends on the thickness of each quartz radiator according to Eqn. 3.2. A thickness of $1 \mathrm{~cm}$ was found to be sufficient, through Monte Carlo simulations. Details of the design and testing of the scanner detector are discussed in Sections 3.5-3.8. The specifications of the final design are given in Section 3.9.

\subsubsection{Linear Motion Assembly}

The scanner detector assembly is mounted on a movable table capable of scanning over the fiducial area of the bottom element of the main detector (see Fig. 3.6) and measuring the spatial distribution of the electron rate. The scanner PMT output pulses are discriminated and counted in scalers to generate rate information, which is correlated with the position information provided by the $2 \mathrm{D}$ motion system in order to generate event rate maps.

The 2D linear motion system consists of two stainless-steel ball-screw driven tables and has a motion range of $200 \mathrm{~cm} \times 26 \mathrm{~cm}$, which extends beyond the fiducial area of the main detector $(200 \mathrm{~cm} \times 18 \mathrm{~cm})$, with good position resolution $(\sim 100 \mu \mathrm{m})$ and minimal skewing. To avoid interference with scattered electrons that could generate helicity dependent asymmetries, non-magnetic material was used as much as possible. DC brushless motors were employed in the system to avoid 
EM interference with other subsystems in the Q-weak experiment, such as the main detectors.

One of the most important design considerations for this motion system is the synchronization of the position information with the detector rate information. This synchronization is realized by reading out position and rate information simultaneously by the same fast DAQ system. Different scan patterns can be used to address different physics questions.

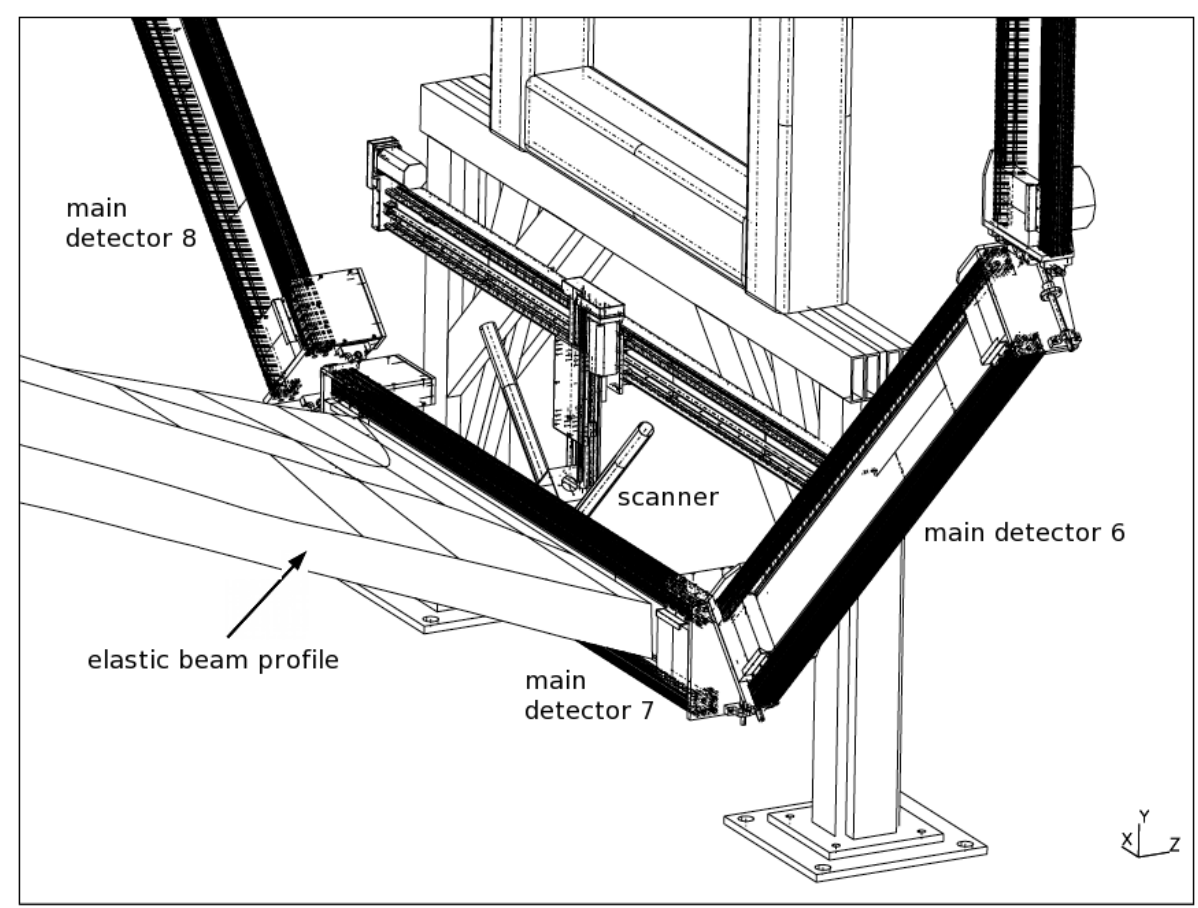

Figure 3.6: CAD model of the scanner system showing the scanner detector mounted downstream of the main detector in bottom octant [99]. The elastic beam envelope is shown as well.

The 2D motion assembly was designed so that it could be mounted in any octant in principle, but eventually only the bottom octant was selected for simplicity of design. The scanner detector is attached to the carrier board of the 2D linear motion assembly with an aluminum support structure. The final design and construction of the motion system will be further discussed in section 3.10. 


\subsection{Monte Carlo Simulations of the Scanner Detector and Backgrounds}

Details of the scanner detector design were studied through extensive Monte Carlo simulations based on the Geant4 toolkit $[100,101]$. The scanner was modeled using various geometries and materials, and the related physics processes, such as Čerenkov radiation, scintillation, multiple scattering, bremsstrahlung, etc., were implemented.

\subsubsection{Benchmarking to E158}

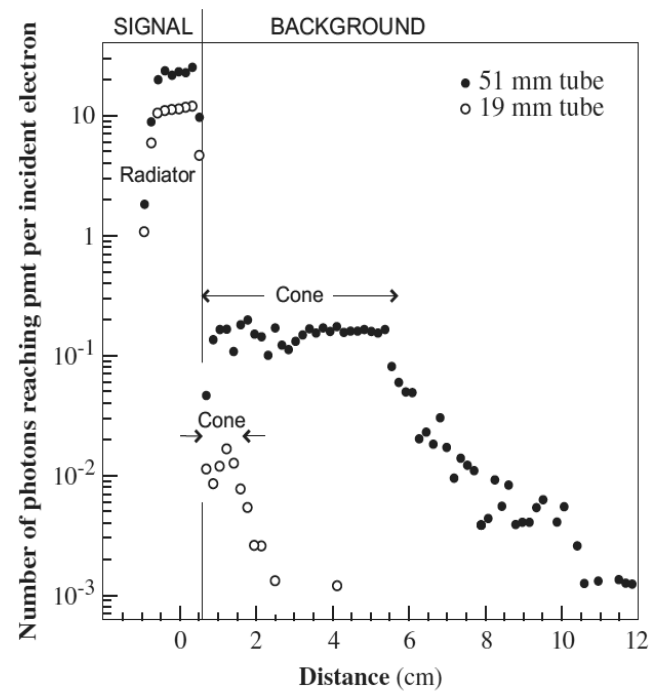

(a)

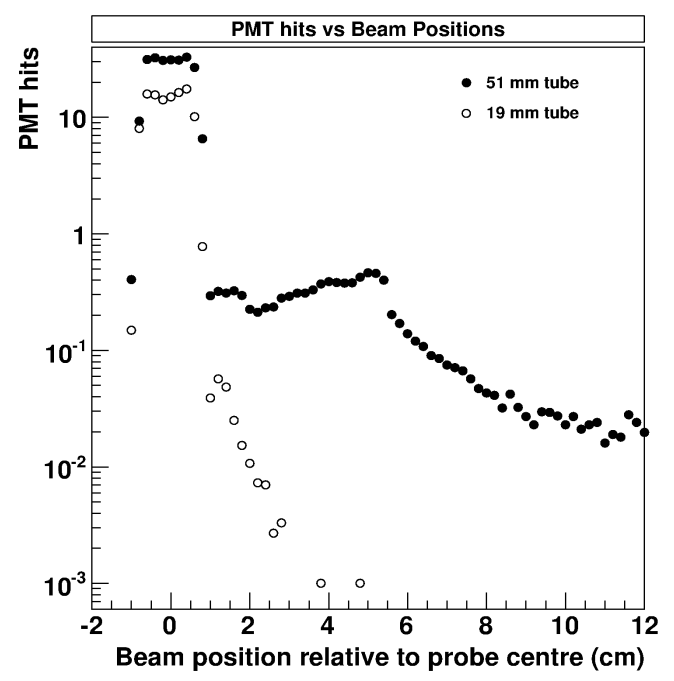

(b)

Figure 3.7: (a) One of the simulation results from E158 scanner paper [94]. (b) Benchmarking the Q-weak scanner simulation results against E158. Both these two plots show the number of photons reaching the PMT per incident electron versus the beam position relative to the centre of the quartz radiator. In this simulation, the PMT is located at $50 \mathrm{~cm}$ from the radiator; the quartz radiator size is $0.5 \mathrm{~cm} \times 0.5 \mathrm{~cm} \times 2 \mathrm{~cm}$; only photons in the wavelength range of $300 \mathrm{~nm}-450 \mathrm{~nm}$ were simulated.

Special care was taken to make sure that optical photon processes were simulated properly. To achieve this, the simulation program for the Q-weak scanner was benchmarked against the E158 scanner simulations. Fig. 3.7 shows an example comparison of one simulated result from the Qweak scanner simulation code with that reported in the E158 paper [94]. In this simulation, an 


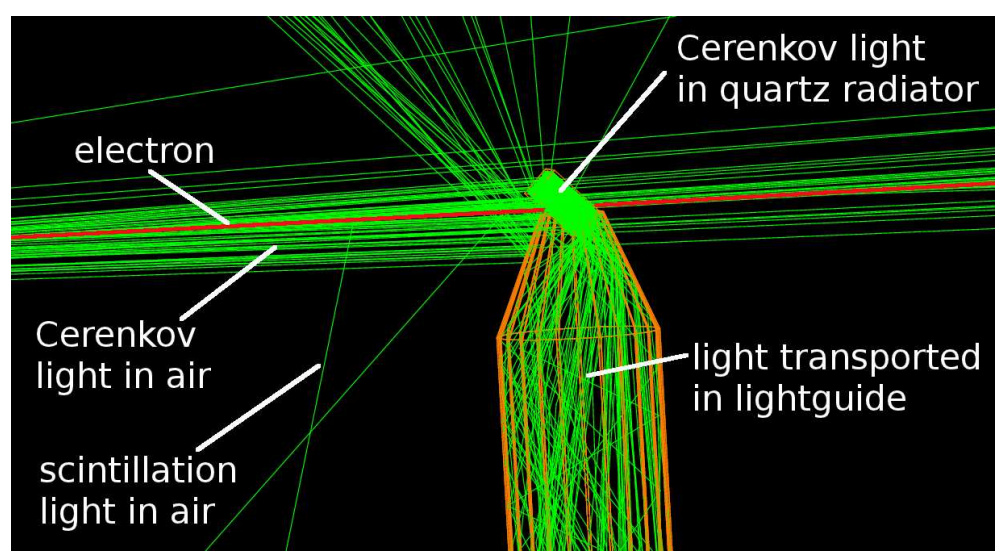

Figure 3.8: Visualization of the scanner simulation for benchmarking to E158.

electron beam was directed toward the model detector at different positions along the axis of the quartz radiator and the air-core light guide (see Fig. 3.8), and the number of hits on the PMT per incident electron was recorded. In these plots, the center of the quartz radiator is defined as the origin, and the various incident beam positions are expressed as distances on the horizontal axis. The responses of detectors with two different light-guide diameters (19 $\mathrm{mm}$ and $51 \mathrm{~mm}$ ) were compared. The simulated signals and background magnitudes for the $51 \mathrm{~mm}$ diameter light guides are also summarized in Table 3.1, which demonstrates basic agreement between the two simulations, although the PMT hits were little higher in ours than E158's. The small discrepancies may arise from different simulation conditions. In E158, the dominant Čerenkov radiation in the air was simulated, while neglecting the scintillation process. In our simulation, both processes were implemented, therefore, our simulated background signals appear slightly larger than those from E158. In addition, in the E158 paper, there is no information available for the optical surface properties of the quartz radiator, PMT window and PMT photocathode. In our simulation, these optical parameters were described according to the properties of the quartz material and PMTs used in Q-weak. These effects should account for the differences of simulated signals between the two packages.

From Fig. 3.7, it can be seen that when the electron beam strikes the radiator, relatively large signals are obtained. When the electron beam strikes the air-core light guide, the PMT rates are 


\begin{tabular}{l|cc}
\hline \hline Beam location & E158 scanner & Q-weak scanner \\
\hline Radiator region & 25 & 30 \\
Cone section (1 - 5 cm to radiator) & 0.15 & 0.3 \\
Straight section (8 cm to radiator) & 0.008 & 0.07 \\
\hline \hline
\end{tabular}

Table 3.1: Comparison of the simulated number of photons reaching a PMT per incident electron for $51 \mathrm{~mm}$ diameter light guides.

about two orders of magnitude smaller. Relatively larger backgrounds originate in the cone-shaped light-guide region, which is adjacent to the radiator, as shown in Fig. 3.3. When beam strikes the straight light guide region, the PMT rates decrease with increasing distance from the radiator. These plots also demonstrate that with the larger diameter of the reflective tube, a larger signal $(\sim$ a factor of 2 increase) is obtained, but with a lower S/B ratio (about an order of magnitude decrease). Based on these results, E158 selected to use $19 \mathrm{~mm}$ diameter light guides for their scanner.

\subsubsection{Geometry and Material Studies}

Using the benchmarked simulation program, various geometries and materials for the Q-weak scanner detector were explored to optimize the design. High light yield is a desirable feature, which requires both sufficient light generation and efficient transport to the PMTs. Light generation was first estimated based on analytical calculations. Simulation techniques were then used to quantitatively understand the light transport process and the variation of light yield with design parameters. Fig. 3.9 shows an example of the predicted PMT hits as a function of the cone angle for the initial conical light guide section. The rates are highest in the range of $\sim 40^{\circ}-55^{\circ}$. Based on this, the angle $45^{\circ}$ was selected in the Q-weak scanner design for easier construction, and based on a better match with the Čerenkov cone angle in quartz for $\beta \sim 1$ particles. To minimize light loss during the transport process, the effects of radiator wrapping materials, the light guide geometry and lining materials, and PMT selections were all systematically studied in simulations. Detailed configurations for each component of the scanner detector will be discussed in section 3.9. 


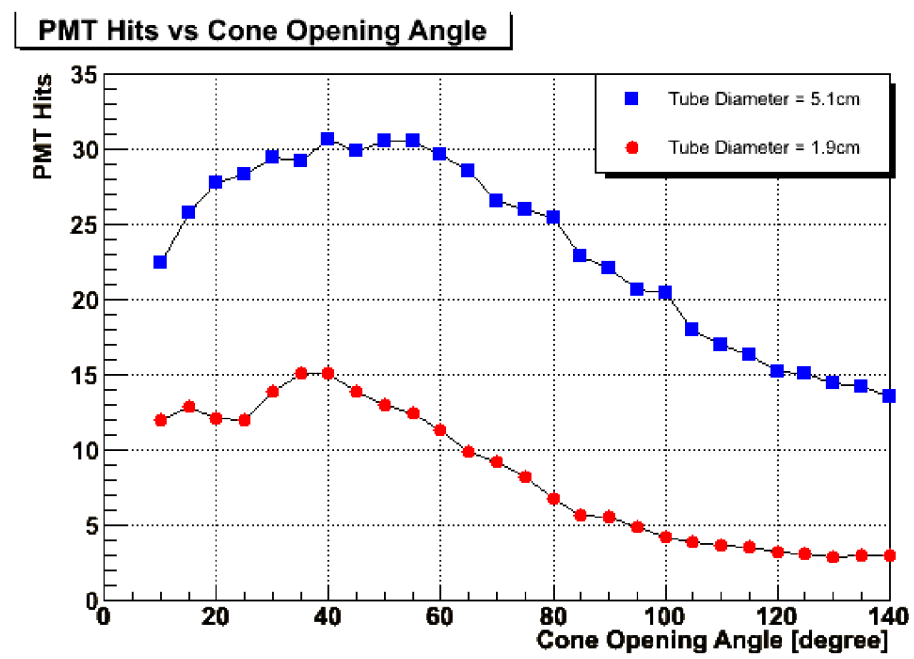

Figure 3.9: Simulated PMT hits versus cone opening angle of light guide for $1.9 \mathrm{~cm}$ and $5.1 \mathrm{~cm}$ tube diameters for the Q-weak scanner.

\subsubsection{Signal}

Simulated light production in the scanner detector indicates that each electron with energy of $1.16 \mathrm{GeV}$ generates about 200 photons within a wavelength band of $300-450 \mathrm{~nm}$ in $1 \mathrm{~cm}$ thick quartz. After reflection losses along the air-core light guide, about 45 photons reach the PMT photocathode. For a typical photocathode quantum efficiency (QE) of $\sim 20 \%-25 \%$, about 10 photoelectrons (PE) would then be sensed in the PMT. This signal magnitude is sufficient for the scanner detector because it is operated in counting mode, and since background pulses with an amplitude of about 1-2 PE can be discriminated from the 10 PE signals with relatively good efficiency and with small contamination.

In addition to producing sufficient signal from the electrons of interest, another major concern in the scanner design is how to reduce detector backgrounds arising from interactions in the light guides of both the conical and straight sections. The simulation for benchmarking to E158 (see Fig. 3.7) demonstrates a signal-to-background (S/B) ratio of about 100, which does not include the background contributions induced by the Q-weak environment. In that simulation, the electron beam was simulated to impinge perpendicularly on the surface of the radiator or light guide. 
However, as discussed in Section 3.3, the Q-weak scanner is subjected to electrons with a range of incident angles, which may enhance edge effects in the quartz radiators and vary backgrounds in light guides. More detailed studies (see discussions in Section 3.8) indicate that in the Q-weak environment, the scanner has a S/B ratio of about $5-20$, where the signal amplitude is about $10 \mathrm{PE}$, and the backgrounds from light guides are less than $2 \mathrm{PE}$.

One way to increase both signal and signal-to-background ratio is to install a pre-radiator for the quartz radiators. A pre-radiator is made of high- $Z$ material so that a high energy electron beam will initiate an EM shower in it. In the scanner simulation code, a pre-radiator was implemented, and its effects were studied. In practice, a $2 \mathrm{~cm}$ thick lead pre-radiator, corresponding to $\sim 4$ radiation lengths, was installed in front of each main detector; that same pre-radiator also serves as a preradiator for the scanner when the scanner is mounted downstream of the main detector.

\subsubsection{Light-guide Associated Backgrounds}

The backgrounds for the scanner detector include all contributions to the scanner signal from interactions occurring anywhere outside the small quartz radiators. From simulations, the dominant background for the scanner detector was expected to come from Čerenkov and scintillation processes in the air volume of the light guides. Compared with the Čerenkov light generated inside the quartz, light yields from the air in the light pipe are small (see Fig. 3.7 (b)). A backup option to reduce the light-guide backgrounds was to fill the guides with a gas with lower scintillation yield (e.g. $\mathrm{CO}_{2}$ ), though this did not prove to be necessary.

In the Q-weak environment, the scanner light guides were exposed to a high intensity gamma ray beam (referred to as the "death ray") generated from beam interactions upstream of the shield house. Unexpectedly high singles rates due to the "death ray" were found (for a detailed discussion, see section 5.1.1). The two PMT coincidence requirement for the scanner events substantially reduced its sensitivity to the high intensity gamma background. 


\subsection{Cosmic Ray Testing of Prototype Detectors}

Based on detector performance simulations, three scanner prototypes, including one single-arm and two double-arm detectors with different configurations, were designed and constructed at The University of Winnipeg, University of Manitoba and TRIUMF. They were initially tested using cosmic rays at the Subatomic Physics Detector Laboratory at the University of Winnipeg.

The basic setup for the cosmic ray tests included two scintillating paddles, the scanner optical assembly, and related electronics, as shown in Fig. 3.10 (a). The scintillating paddles were located $\sim 20 \mathrm{~cm}$ above and below the sensitive components of the scanner to define the paths of cosmic ray muons that would pass through the quartz radiators. The scintillators provided a gate signal for a charge sensitive ADC, which digitized the PMT output pulses.

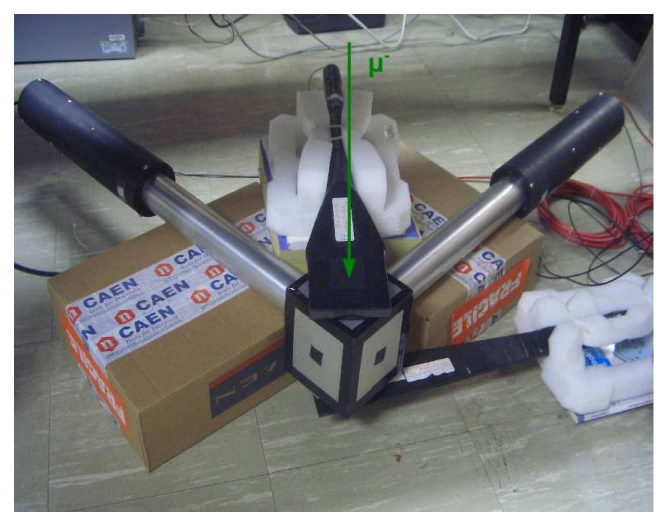

(a)

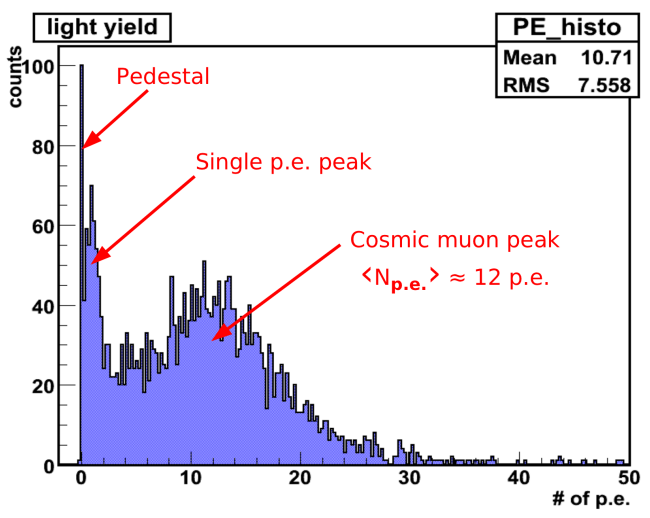

(b)

Figure 3.10: (a) The scanner detector cosmic ray test set up: the upper and the lower scintillator paddles were used to define cosmic muon directions and to provide a coincidence gate for the scanner detector signals. (b) The scanner PMT's pulse height spectrum from cosmic ray tests. Single photo-electron (PE) events were used to calibrate the PMT output signals, and the muon peak appears to be larger than $10 \mathrm{PE}$.

Fig. 3.10 (b) shows a typical pulse height spectrum from one PMT of a scanner prototype. There are three peaks appearing in the figure. The large sharp peak on the left side is the pedestal peak, corresponding to events that missed the active element of the detector because of the much 
larger trigger paddle size relative to the quartz radiator size. The wide peak on the right side is the cosmic muon peak due to Čerenkov radiation by muons in the quartz. The narrow peak in between, referred to as the single photo-electron (PE) peak, is mainly caused by the thermal noise from the PMT photocathode, edge events, and low-energy muons. The PMT output signals were calibrated to the number of photo-electrons using the $1 \mathrm{PE}$ peak and the pedestal peak in the spectrum. The test result for the detector photo-electron yield demonstrates good consistency with the Geant4 simulations (see the simulated spectra in Fig. 3.16 for reference).

Extensive tests were conducted with the prototypes. For example, the light yield using different sensitive components, such as scintillator, acrylic and quartz, was tested. PMTs with various entrance windows and gains were tested. Tests of various light guide materials, including Anolux, Anomet, "MIRO-2" and "MIRO-4" [102], were also performed; about 5 - 30\% differences in PMT yield were observed. The "MIRO-4" specular aluminum sheet with a total reflectivity of larger than 95\% showed the best signal and was therefore used in the final scanner optical assembly.

\subsection{TRIUMF Beam Tests}

\subsubsection{Signal and Comparison to Simulations}

Beam tests of the final scanner prototype were performed at TRIUMF in the summer of 2008. The tests were conducted in the M11 pion channel using a mixed beam of pions, muons and electrons. Electrons were separated from the pions and muons using a time of flight (TOF) technique with two trigger scintillators in the experimental area (see the test setup in Fig. 3.11 and the TOF spectrum in Fig. 3.12). The beam momentum was selected by a large dipole magnet and collimator system. Good electron rates $(\sim 800 \mathrm{~Hz} \mathrm{~S} 1$ trigger rate; 20 - $40 \mathrm{~Hz}$ coincidence rate from S1 and S2 trigger paddles) and particle identification could be achieved for $p=120 \mathrm{MeV} / \mathrm{c}$, and therefore that momentum setting was used.

Several different quartz configurations with $1 \times 1 \mathrm{~cm}^{2}$ fiducial area were compared. Fig. 3.13 shows the scanner pulse height spectra from three types of configurations. The configuration (a) with 


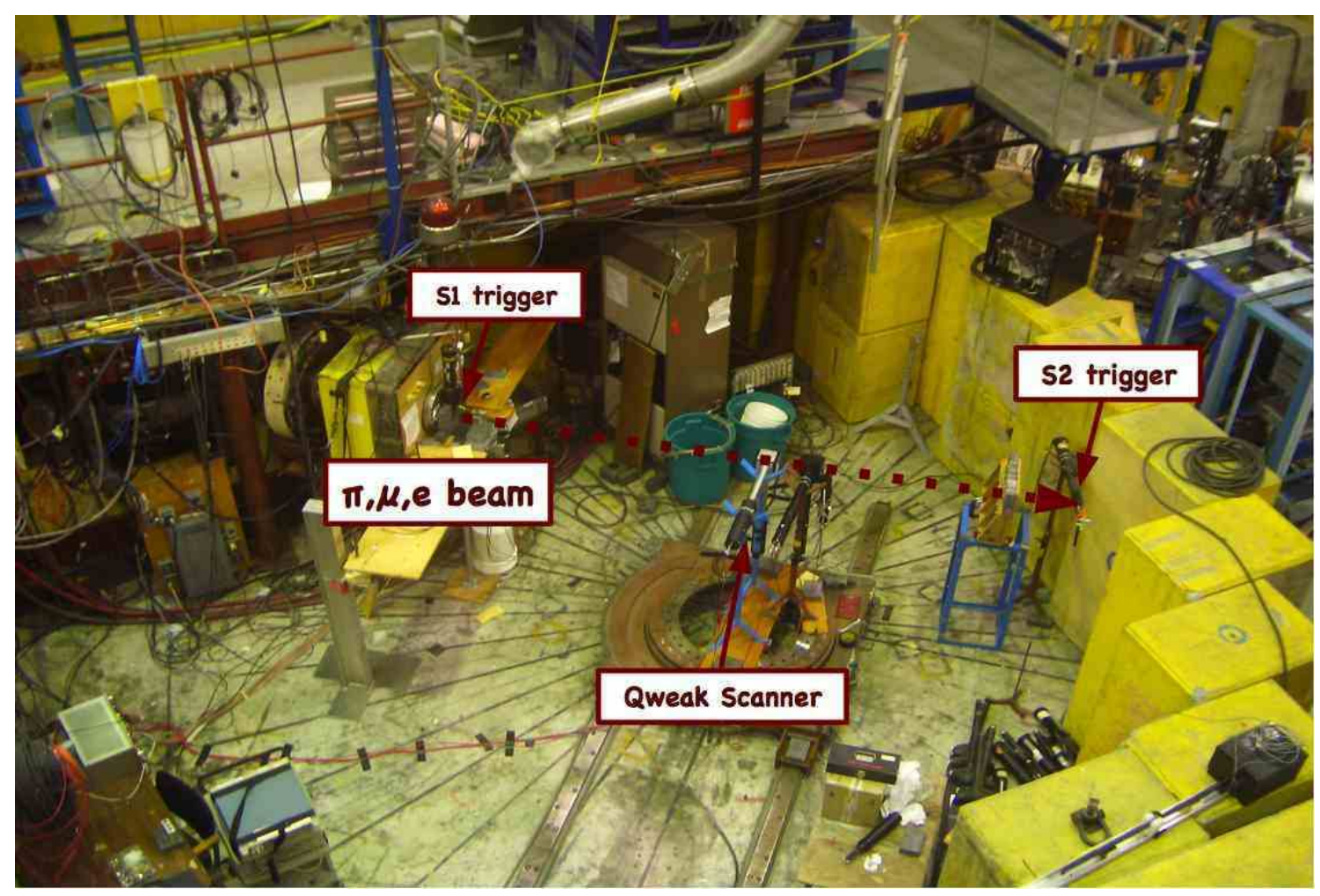

Figure 3.11: TRIUMF beam test setup. The beam entered the M11 area from the left side, being selected by two trigger scintillator paddles located about $10 \mathrm{~m}$ apart from each other. The scanner prototype was installed between the two scintillator paddles.

two $1 \times 1 \times 2 \mathrm{~cm}^{3}$ optically decoupled radiators was the baseline version at that time; however, it had the disadvantage that the light yield arising from a quartz radiator beyond its fiducial area would contribute large background signals to the detector, and affected the subsequent rate measurements. Compared with (a), configuration (b) with two $1 \times 1 \times 1 \mathrm{~cm}^{3}$ decoupled radiators obtained a similar light yield ( $\sim 10 \mathrm{PE})$ and lower backgrounds, but was more sensitive to the electron's incident angle in $\theta$ and $\phi$. The configuration (c) with one piece of $1 \times 1 \times 1 \mathrm{~cm}^{3}$ quartz coupled to both PMTs contributes lower light yield ( 5 - 7 PE) to single PMT, with low backgrounds, and lower sensitivity to tilt angle; however, there is more risk for background light to be transported between the two tubes. Based on these comparisons, configuration (b) was selected. It was confirmed later that the sensitivity to electron's incident angle of configuration (b) did not strongly affect the scanner's rate 


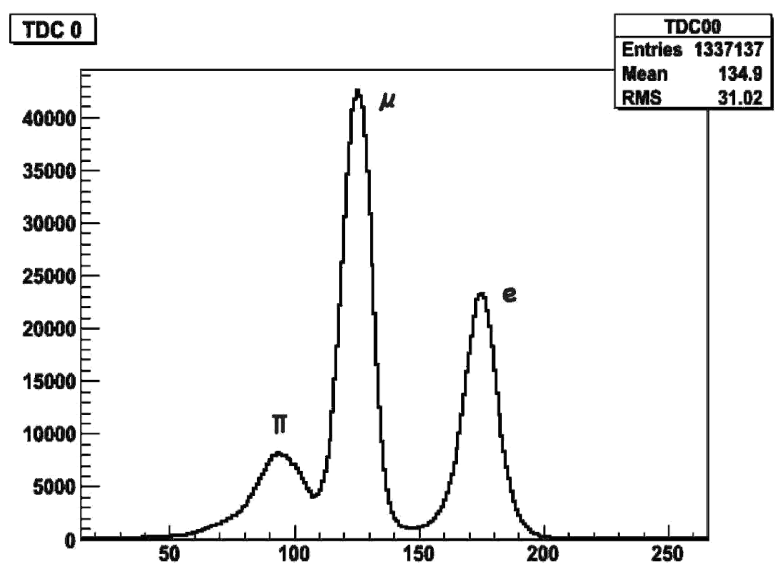

Figure 3.12: The TOF spectrum for particle identification in the pion, muon and electron beam. The horizontal scale is time in arbitrary units.

measurements, as will be discussed in Section 3.8.

From the beam tests, the scanner signal yield of $\sim 10$ PE per incident electron was calibrated and confirmed to be consistent with the results from simulations and cosmic tests. For configurations (a) and (b), five sides of each piece of quartz were covered with wrapping materials, only the sixth being open to the light pipe; for configuration (c), four sides of the quartz were covered, the other two sides being open to two light pipes, respectively. According to simulations and cosmic tests, the radiator-wrapping materials were expected to affect the light yield at the $10 \%-15 \%$ level relative to the case with no wrapping. In beam tests, effects due to different wrapping materials, including Tyvek, white paper and Teflon, were studied. As shown in Table. 3.2, about 10 - 15\% variations were found, which was most likely because the quartz used for testing were not well polished. The imperfect total internal reflection (TIR) indicates the importance of "good" wrapping with a material of high reflectivity.

In the PMT spectra in Fig. 3.13, the events between the single PE peak and the wide electron peak are partially due to quartz edge effects and the backgrounds arising from the conical section of light-guides. Tests of the scanner's sensitivity to tilt angles $(\theta, \phi)$ with different quartz configurations and threshold settings were performed to vary the contribution of edge effects in the detector signal. 


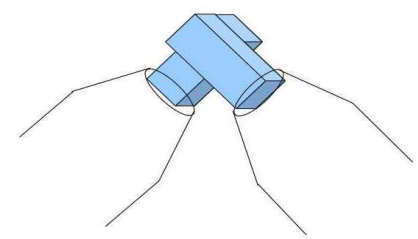

(a) with two $1 \times 1 \times 2 \mathrm{~cm}^{3}$ quartz

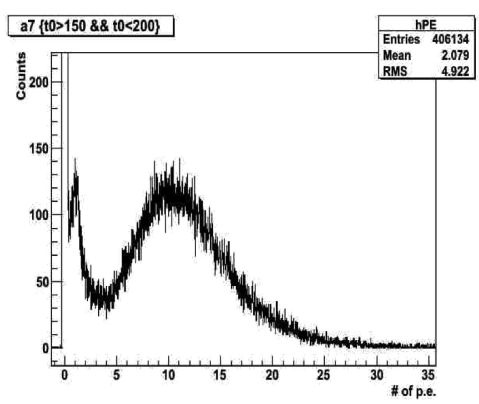

(d) with two $1 \times 1 \times 2 \mathrm{~cm}^{3}$ quartz

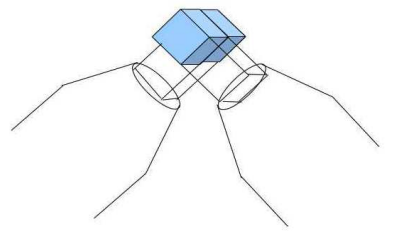

(b) with two $1 \times 1 \times 1 \mathrm{~cm}^{3}$ quartz

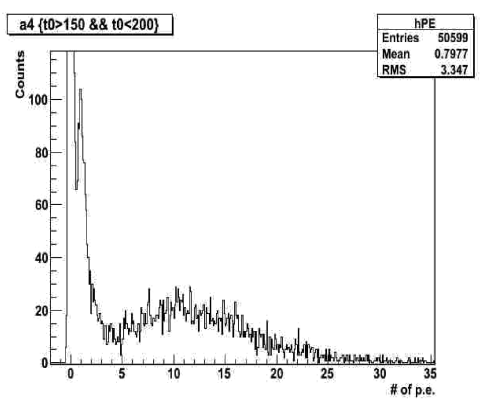

(e) with two $1 \times 1 \times 1 \mathrm{~cm}^{3}$ quartz

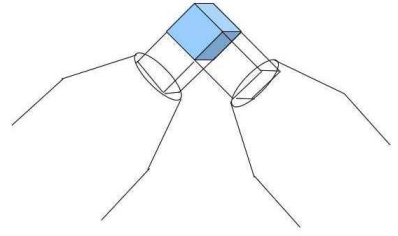

(c) with one $1 \times 1 \times 1 \mathrm{~cm}^{3}$ quartz

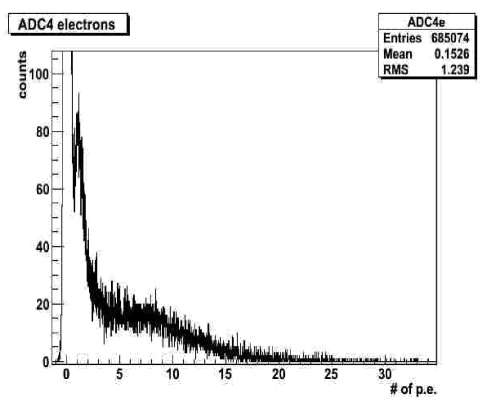

(f) with one $1 \times 1 \times 1 \mathrm{~cm}^{3}$ quartz

Figure 3.13: Comparisons of scanner pulse height spectra (d), (e) and (f) from TRIUMF beam tests with three different quartz configurations illustrated in (a), (b) and (c), respectively. The blue blocks represent the quartz radiators, which are wrapped with Tyvek so as to couple Čerenkov light into the corresponding light guide with low light loss. Configuration (a) receives higher rate due to larger radiators compared with other configurations; configuration (b) obtains the same light yield as configuration (a), but lower rate than (a); configuration (c) has the lowest light yield since the light generated in one quartz radiator is shared by two separate light guides.

In the Q-weak environment, the scanner detector outputs represent a response to a complicated function of many variables, including the electron's incident angles, detector positions, threshold setting, etc. Therefore, a full Monte Carlo simulation of the Q-weak environment was needed in order to assess the anticipated position-sensitivity of the scanner under realistic conditions, as discussed in Section 3.8. 


\begin{tabular}{cc}
\hline \hline Wrapping material & Light yield (number of PE) \\
\hline Tyvek & $10.7 \pm 0.2$ \\
white paper & $8.7 \pm 0.4$ \\
Teflon tape & $9.4 \pm 2.4$ \\
\hline
\end{tabular}

Table 3.2: Comparison of light yield with different radiator-wrapping materials for the configuration (b) shown in Fig. 3.13.

\subsubsection{Background Light Yield}

Besides the detector signal test, the detector backgrounds were also tested with the TRIUMF beam. During background tests, the quartz radiators were removed. The detector position was varied so that the beam would strike different positions along the axis of one air-core light guide, from the cone region down to the cylindrical region near the PMT.

Fig. 3.14 shows a comparison of background measurements at the cone region and the middle of the cylindrical region, respectively. It can be seen that the background from the cone region of the air-core light guide is larger than that from the straight part, as indicated by the previous simulations. For both cases, the background signal amplitudes are at the $1 \mathrm{PE}$ level. These results suggest that the threshold for the scanner accepting pulses should be set above 1 PE in order to separate the light-guide related backgrounds from true signals.

\subsection{Monte Carlo Simulations of Scan Efficiency}

The fiducial area of the scanner detector, formed by the geometrical overlap of the two quartz radiators, is a function of the electron's incident angles, scan positions, and threshold setting, expressed by $A(x, y, \theta, \phi$, threshold). Since electrons enter into the radiators from different directions at various positions, the fiducial area may change from one position to the other, consequently leading to changes in the detector efficiency. The uniformity of the scanning detector response in the Q-weak experimental environment was therefore explored in Monte Carlo simulations. 


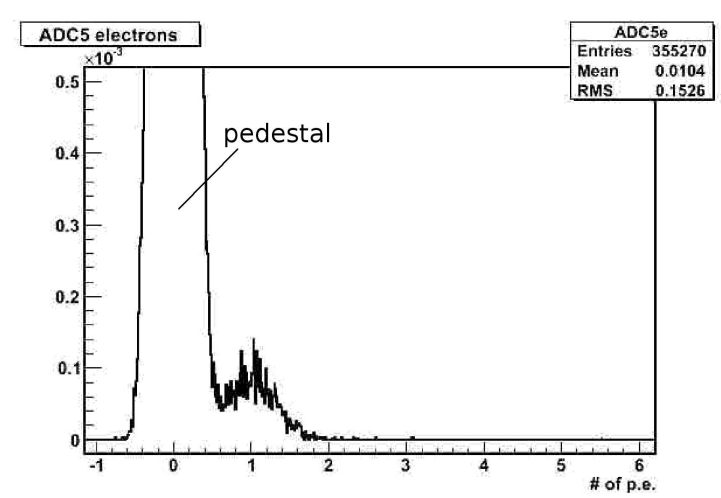

(a)

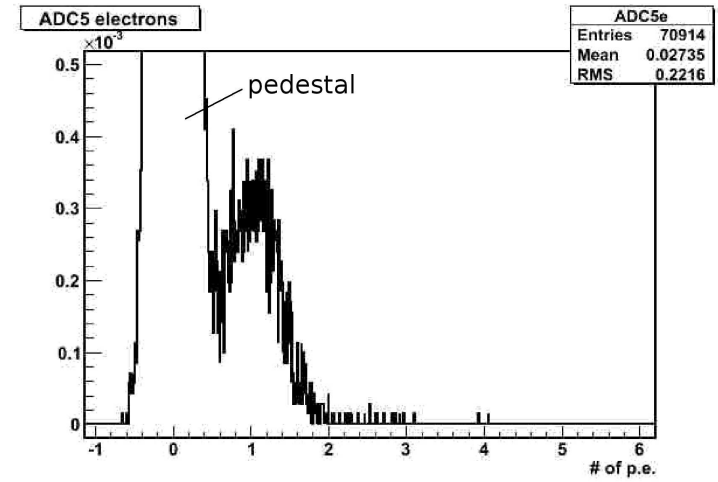

(b)

Figure 3.14: Scanner backgrounds in air-core light guides (both histograms were normalized to the total number of incident electrons): (a) beam incident on the middle of the straight light-guide area, (b) beam incident on the cone area. In each histogram, the signal peak on the right side indicates the light yield strength of $\sim 1 \mathrm{PE}$.

For this purpose, the previous scanner Geant4 simulation package was extended to the Q-weak environment. The hit pattern of elastic electrons impinging on the main detector obtained in a global Q-weak Geant4 simulation was used as an event generator for the scanner simulation. Fig. 3.15 shows a visualization of the scanner simulation in Q-weak optics. The scanner detector model with two $1 \times 1 \times 1 \mathrm{~cm}^{3}$ quartz radiators was coded to move step by step automatically and measure the rate distribution in that $z$-plane. In this package, the center of the event generating area (the fiducial area of the main detector) is defined as the origin of the coordinate system. The geometric center of the scanner radiators varies over the range of $x=[-100 \mathrm{~cm}, 100 \mathrm{~cm}]$ and $y=[-10 \mathrm{~cm}, 15 \mathrm{~cm}]$ in a plane at constant $z$ downstream of the main detector. Via this extended simulation package, it was possible to study the detector's efficiency at different positions in $x, y$ and $z$, and to model potential applications in the Q-weak experiment. Fig. 3.16 shows examples of simulated scanner PMT spectra. The light yield of $\sim 11 \mathrm{PE}$ is in good agreement with TRIUMF beam test results.

A set of studies related to effects of radiator geometry and detector efficiency was performed through simulations. In our consideration, the ideal scanner radiator would be infinitely thin and would have a large light yield. To test the scanner's efficiency, such an ideal detector was used as 


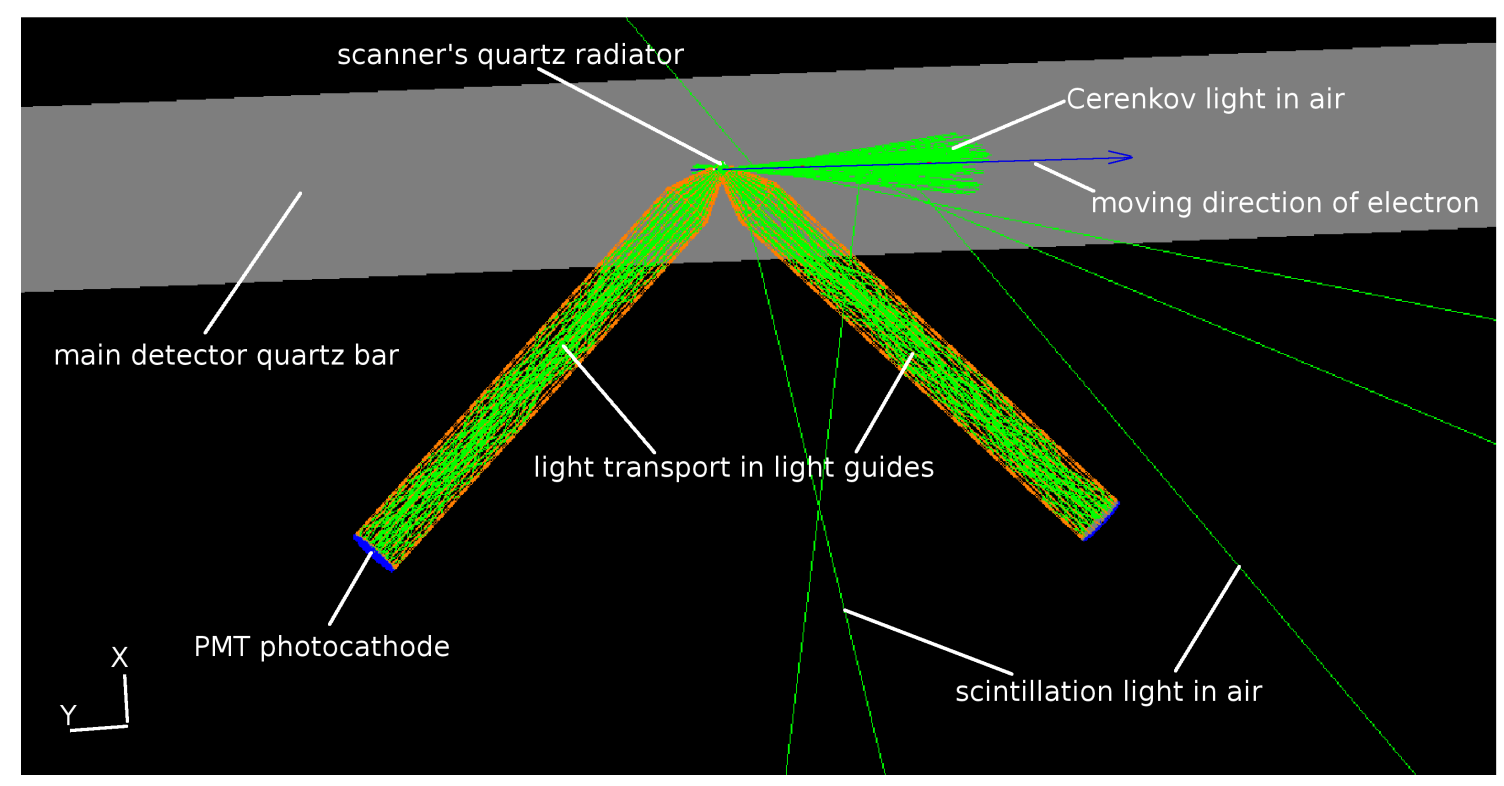

Figure 3.15: The scanner simulation visualization. The gray region represents the main detector quartz bar. The scanner model is located $14 \mathrm{~cm}$ downstream of the main detector, receiving both primary electrons and secondaries $\left(e^{+}, e^{-}\right.$and $\left.\gamma\right)$ from the Čerenkov bar. The green lines represent the Čerenkov and scintillation light that is generated and transported in the scanner quartz radiators and in the air.

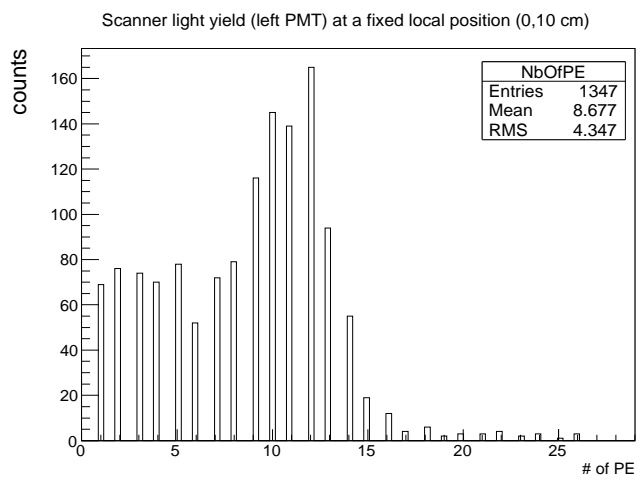

(a)

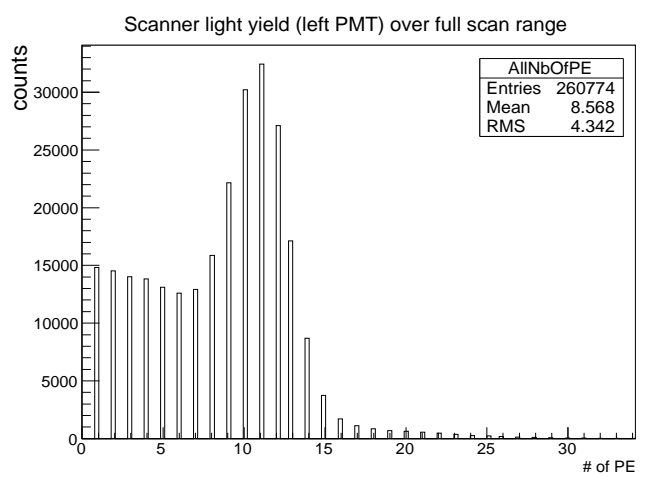

(b)

Figure 3.16: The simulated scanner light yield spectra. (a) One PMT (left) spectrum at a fixed local position $(0,10 \mathrm{~cm})$. (b) One PMT (left) spectrum over the full scan range. The light yields in the spectra were expressed in units of photo-electron (PE). The low PE contributions are mainly from backgrounds and quartz edge events. 


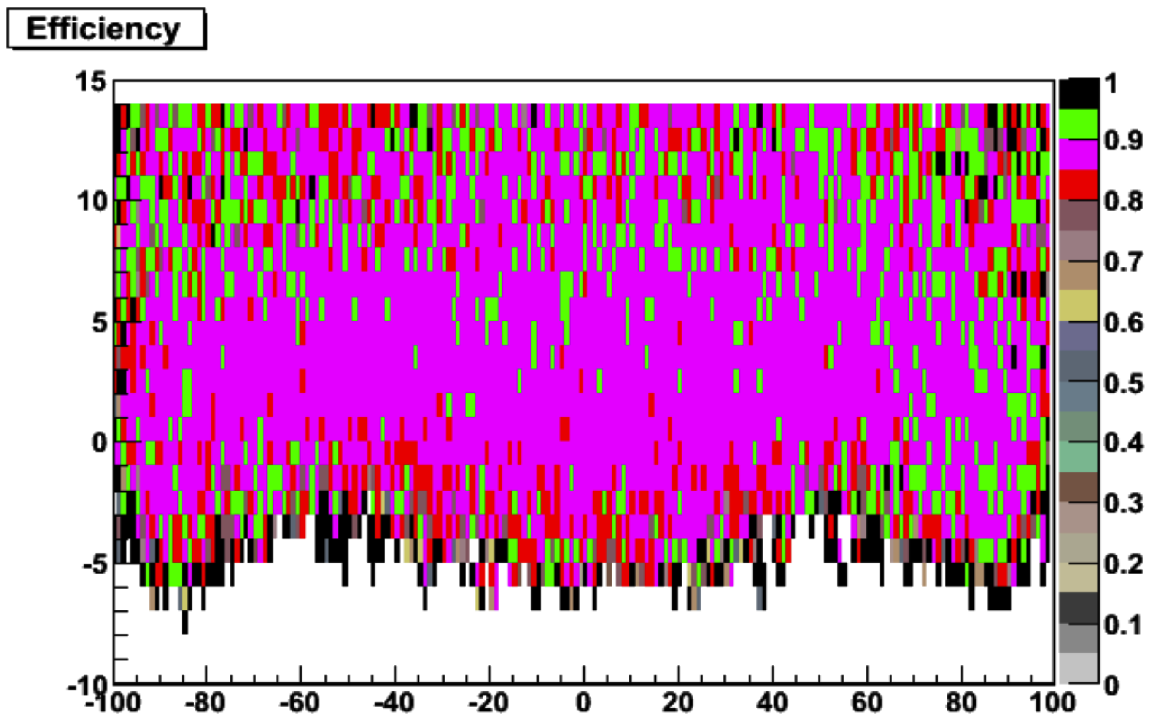

Figure 3.17: The simulated scanner detector efficiency map with threshold setting of $\sim 0 \mathrm{PE}$ at $5 \mathrm{~cm}$ downstream of the main detector. The $x$ - and $y$-axes in units of $\mathrm{cm}$ represent the local coordinates defined in the scanner simulation package. The $x$-axis and $y$-axis are along the length and width of the main detector quartz bar, respectively, with the origin defined as the center of the quartz bar. The color scale indicates the scan efficiency.

a baseline for comparisons, and was defined to have an efficiency of $100 \%$. Detector efficiency in our following discussion is defined as the measured coincidence rate from two PMTs of the scanner detector relative to that from an ideal detector. The resultant efficiency map with a threshold of $\sim 0$ PE is depicted in Fig. 3.17, which demonstrates a fairly uniform ( 90\% - 100\%) efficiency distribution across the entire bar. It is understandable that the detector efficiency would decrease with increasing threshold, as the relative contribution of edge events is reduced. Further investigations were then focused on the uniformity of the efficiency distribution at higher threshold settings. Fig. 3.18 provides an example to address this question, demonstrating how rate distributions and detector efficiencies vary with threshold settings (from 0 to $3 \mathrm{PE}$ ) when the detector scans along the $x=-85 \mathrm{~cm}$ vertical strip. With a threshold of $1 \mathrm{PE}$, the scanner detector has an efficiency of $\sim 80 \%$. Even with a threshold of $3 \mathrm{PE}$, the efficiency is $63 \%$ and uniform to $\pm 7 \%$ absolute. 
Similar studies were performed at different positions, which indicate that for a threshold above $1 \mathrm{PE}$, most of the edge effects in the quartz radiators are removed. The active area of the scanner detector and its efficiency at different positions are fairly uniform over the entire scan area with a threshold above $1 \mathrm{PE}$. This verified that basic needs for detector design could be met with our proposed radiator configuration. However, the higher the threshold, the lower the detector efficiency, so the threshold setting and efficiency requirements have to be carefully balanced in actual applications.

With the extended scanner simulation package, some other effects and applications were also studied. For example, the defocusing effects of the elastic electron envelope due to the QTOR magnetic field were investigated in different $z$-planes to assist in the scanner detector mounting design. Fig. 3.19(b) shows an example of a simulated rate distribution with the scanner model at thresholds of 3 PE for both PMTs, located $14 \mathrm{~cm}$ downstream of main detector. In this rate map, the elastic electron image appears in a "moustache" shape, and is shifted radially $\sim 5.5 \mathrm{~cm}$ relative to that in the main detector plane, as displayed in Fig. 3.19(a). 

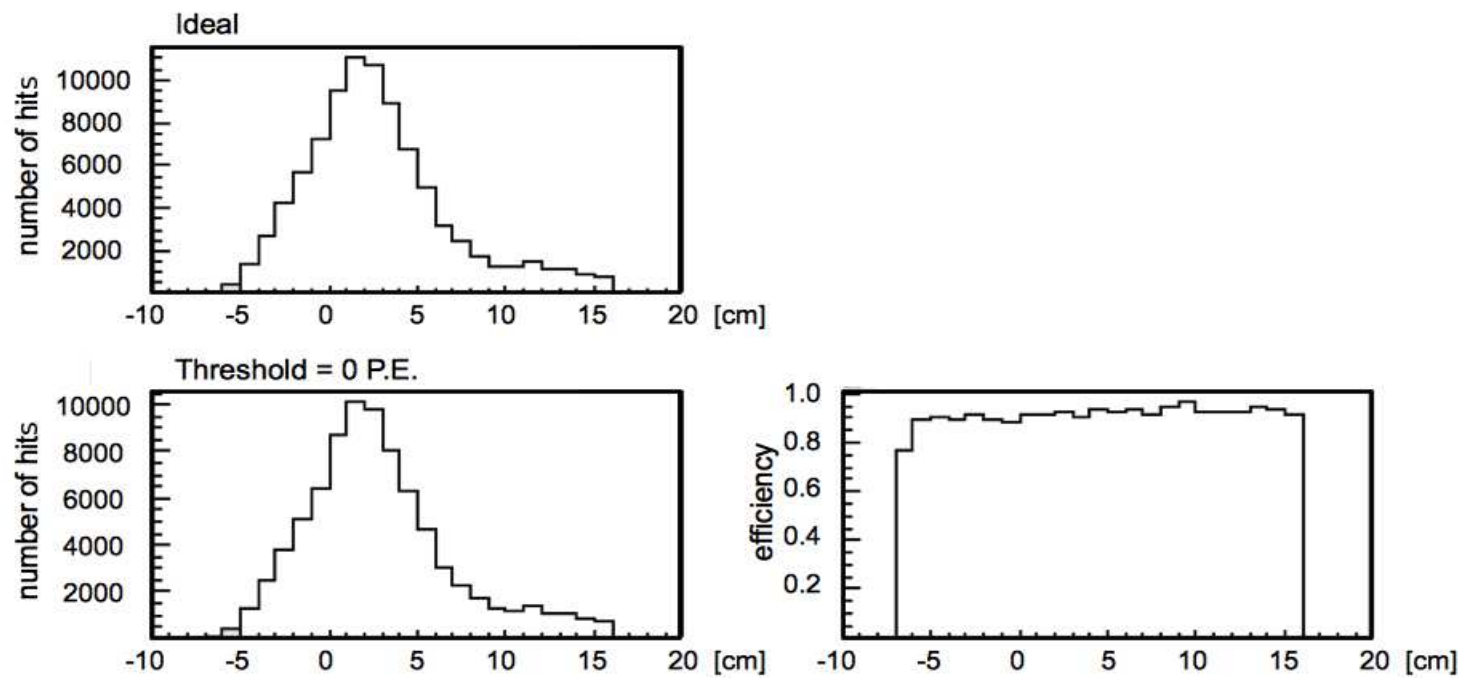

Threshold = 1 P.E.
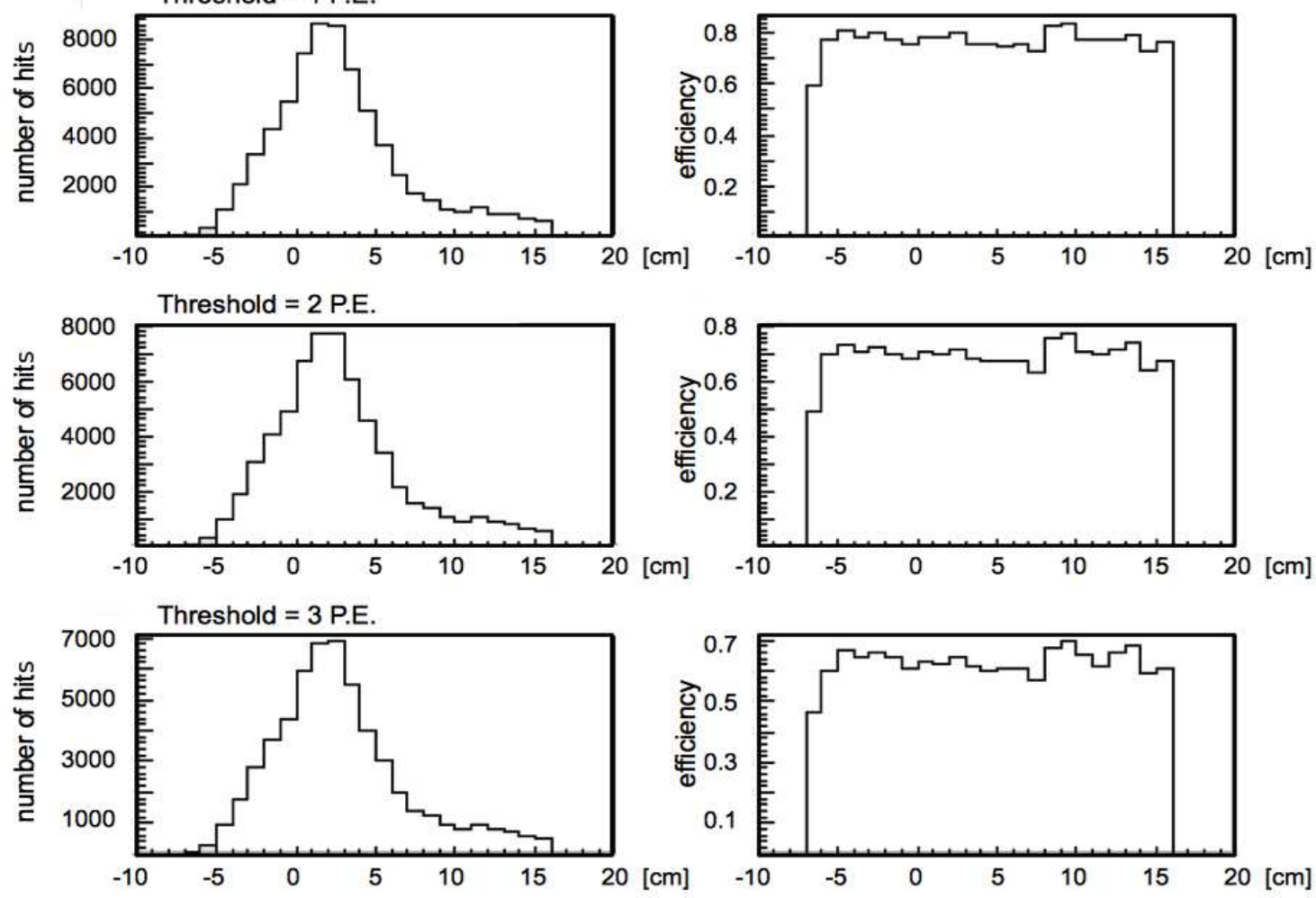

Figure 3.18: Example of the simulated scan efficiency versus threshold setting of 0 to $3 \mathrm{PE}$ when the scanner moves along the $x=-85 \mathrm{~cm}$ strip in the $z$-plane $5 \mathrm{~cm}$ downstream of the main detector. The plots in the left column are rate distributions for the ideal case, and 0 - 3 PE thresholds from top to bottom; the plots in the right column are the corresponding detector efficiencies for 0 - $3 \mathrm{PE}$ thresholds settings, respectively. 


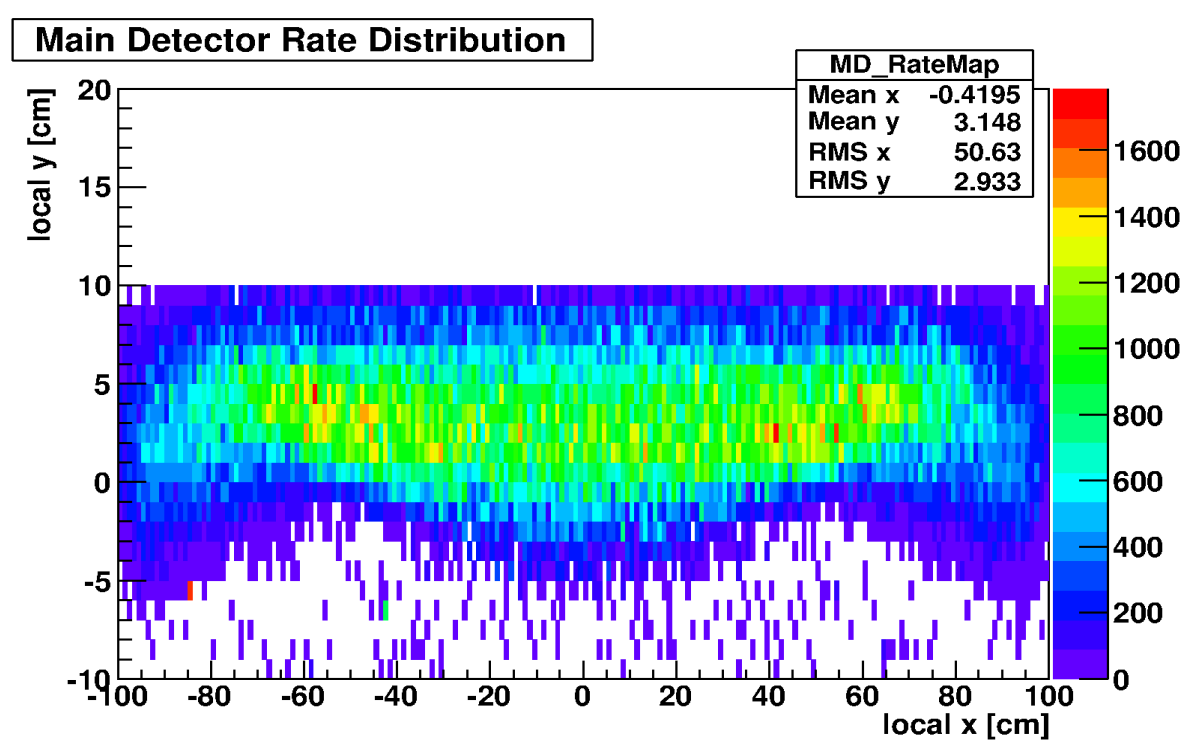

(a) Simulated rate distribution in the main detector plane.

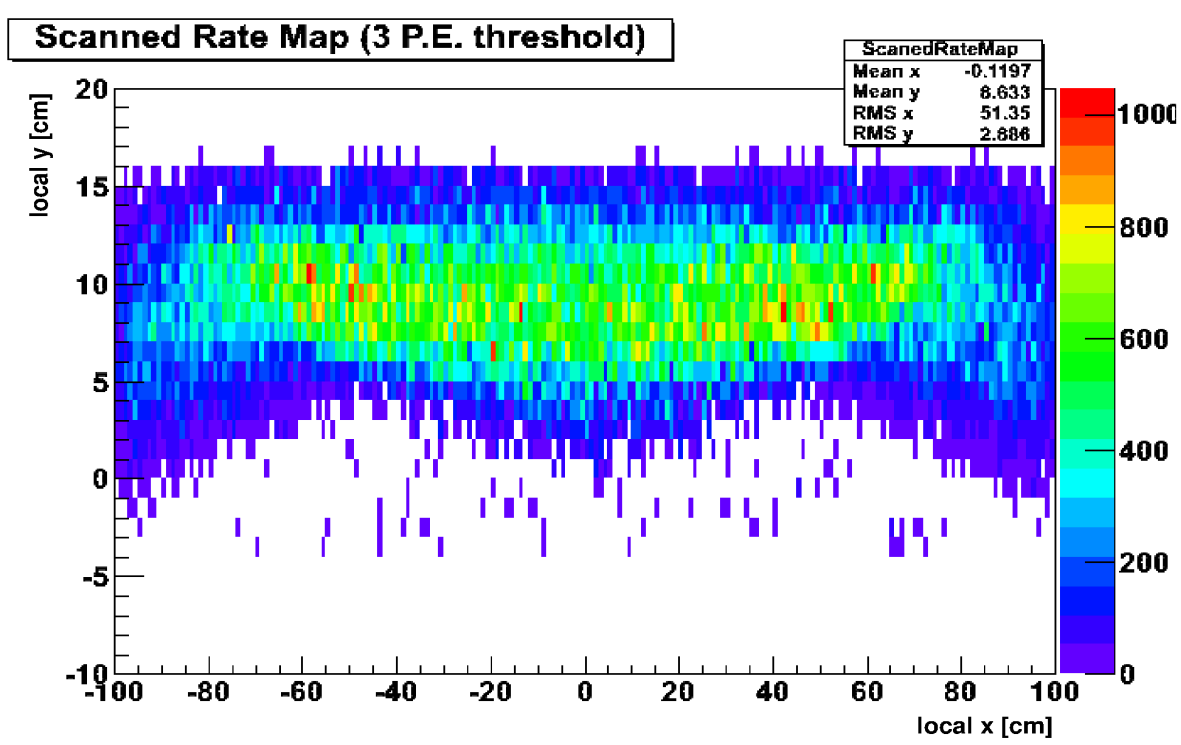

(b) Simulated rate map seen by the scanner detector located $14 \mathrm{~cm}$ downstream of the main detector with $3 \mathrm{PE}$ threshold.

Figure 3.19: A comparison of the simulated rate map seen by the scanner detector with the rate distribution in the main detector plane. Local coordinates were used in this simulation. The elastic electron image seen by the scanner shifts radially $\sim 5.5 \mathrm{~cm}$ relative to that in the main detector plane. The scanner efficiencies of $\sim 60-70 \%$ at 3 PE threshold also can be seen by comparing the color scales, which indicate the rates in the same arbitrary units. 


\subsection{Final Detector Design and Construction}

\subsubsection{Radiator}

As mentioned in the previous section, in the scanner detector, two pieces of $1 \mathrm{~cm} \times 1 \mathrm{~cm} \times 1 \mathrm{~cm}$ synthetic quartz were employed as the active elements. They were obtained from a main detector sample block (Spectrosil 2000 quartz), which was precisely cut with a diamond saw in the NanoSystems Fabrication Laboratory at the University of Manitoba (see the photos of quartz cutting in Fig. 3.20). They were placed one in front of the other, giving a $\sim 1 \times 1 \mathrm{~cm}^{2}$ active area.

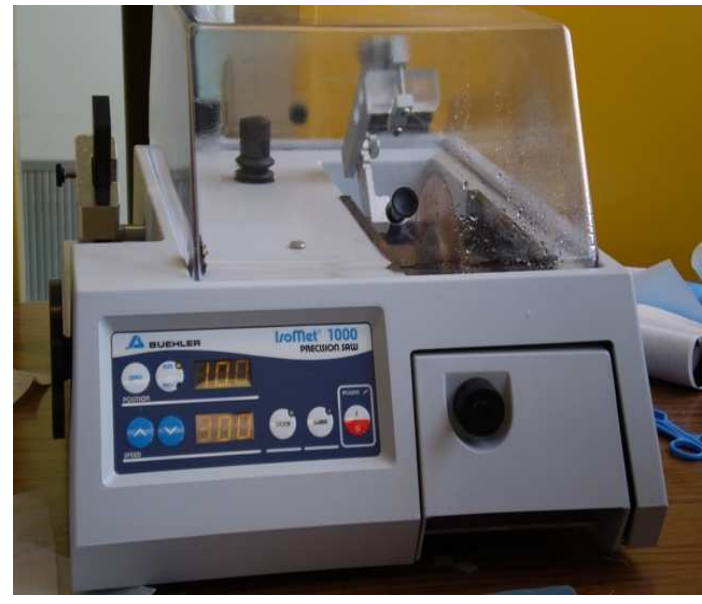

(a)

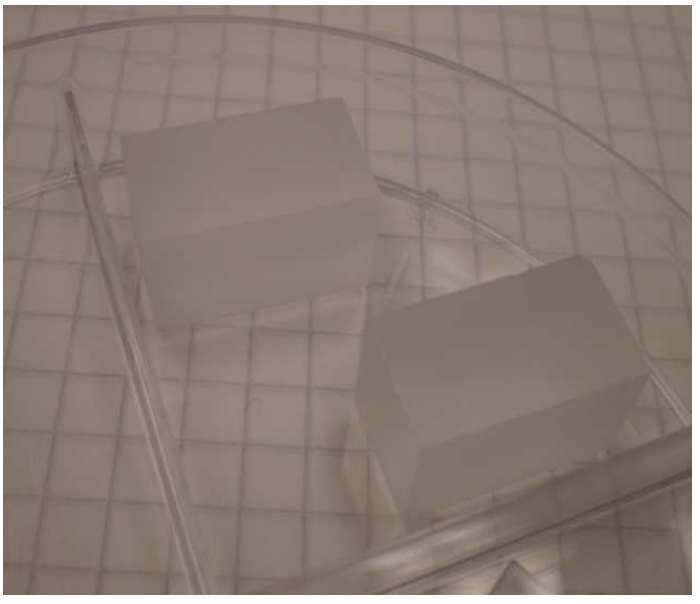

(b)

Figure 3.20: (a) A quartz radiator being cut with a precision diamond saw in the Nano-Systems Fabrication Laboratory at the University of Manitoba. (b) Prototype quartz radiators after cutting. The size of these radiators is $1 \mathrm{~cm} \times 1 \mathrm{~cm} \times 2 \mathrm{~cm}$.

To maximize the efficiency of the light transport, the surfaces of the quartz were fine-polished to $100 \AA$ (rms) so that Čerenkov photons could be transported inside the quartz radiator via total internal reflection with minimal light loss. The polishing was done by hand on a flat plate. Each element was separately wrapped with white Teflon tape, so that the light produced in the two quartz elements was decoupled from each other and sent to an independent air-core light pipe. Each quartz cube was covered on five sides, the sixth being open to the light pipe. 


\subsubsection{Light Guides}

The Čerenkov light from each quartz radiator was directed by means of a reflective air-core lightguide to the PMTs. The light-guides consist of hollow-tubes lined with reflective MIRO-4 Alzak sheet [102] of thickness $0.08 \mathrm{~mm}$. The light pipe lining was rolled with a 51-mm-diameter roller machine. The lining material with an optical finish was produced by electrochemically brightening and anodizing a high purity aluminum alloy. This material has high permanent reflectivity (>95\%) and good resistance to corrosion and abrasion, so the photons can be transported toward the PMTs with minimal light loss. Each light-guide is composed of a cone-shaped section and a straight 51$\mathrm{mm}$-diameter cylindrical section. The $\sim 5 \mathrm{~cm}$ long cone-shaped section is a link for coupling the light from the quartz radiator to the cylindrical section of the light-guide with a small top opening. The half-angle of the cone is $45^{\circ}$, which is well matched with the Čerenkov angle in the quartz and reduces the total number of reflections along the tube, and thus light loss. The small top opening of the cones also minimizes the chance of light escaping. A total length of $50 \mathrm{~cm}$ was chosen for the light-guides. With this length, sufficient light is collected by the PMTs, and both PMTs can be located out of the scattered electron beam profile to reduce radiation damage.

The light from each quartz radiator is directed into its own light-guide and PMT, and is optically decoupled from the other light tube assembly. The coincidence rate of the two PMTs is used to signify the passage of a high-energy electron through both quartz elements. In order to reduce accidental coincidences, the light pipes for the two different radiators are arranged in a V-shape with $90^{\circ}$ between them, as depicted in Fig. 3.22, instead of being in line with the incident beam direction.

\subsubsection{PMTs}

Light produced in the scanner detector is sensed by two $51 \mathrm{~mm}, 12$ stage XP2268 PMTs manufactured by Photonis Technologies [103]. This PMT features a quartz window and bi-alkali (antimonyrubidium-caesium $\mathrm{Sb}-\mathrm{Rb}-\mathrm{Cs}$, antimony-potassium-caesium $\mathrm{Sb}-\mathrm{K}-\mathrm{Cs})$ photocathode, and has high sensitivity to a large spectral range from $150 \mathrm{~nm}$ to $650 \mathrm{~nm}$, with a maximum at $420 \mathrm{~nm}$ (see 
Fig. 3.21). The relatively high window transparency and photocathode sensitivity in the UV band makes this PMT suitable for Čerenkov light collection (refer to Fig. 3.5 for Čerenkov light generation in the UV region). Photonis S563 voltage dividers are attached to these PMTs. With this type of divider, and an applied voltage of $\sim 1900 \mathrm{~V}$, the PMT has a typical gain of $10^{7}$.

\section{Typical spectral characteristics}

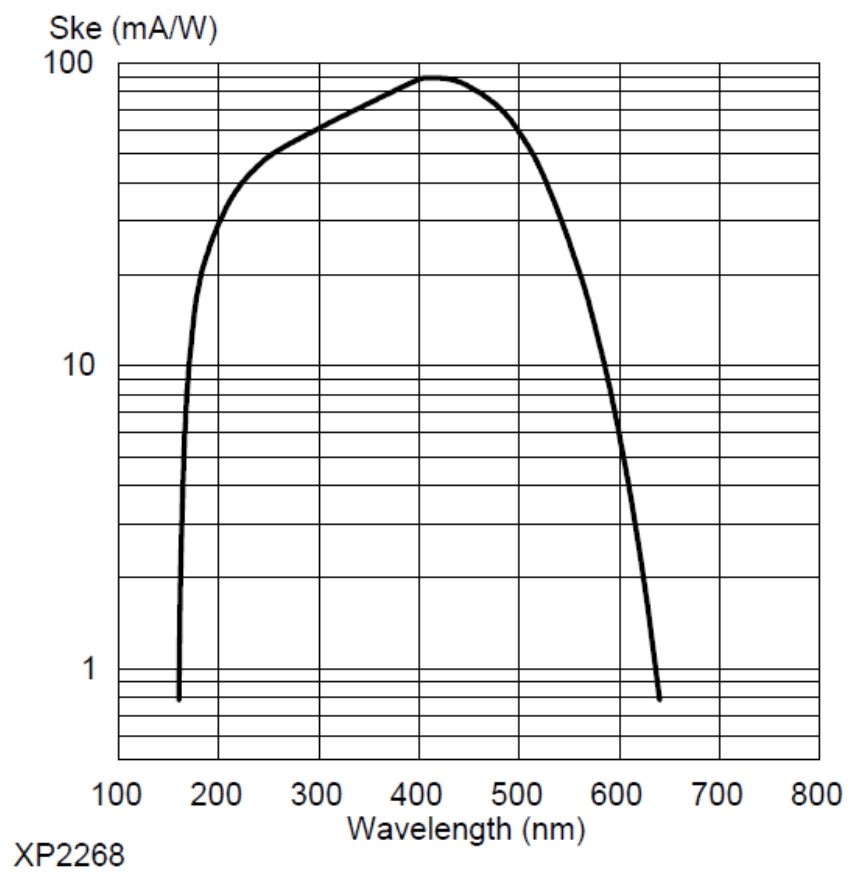

Figure 3.21: Typical spectral characteristics (photocathode sensitivity to photon wavelength) of XP2268 PMTs [103]. The vertical scale indicates the radiant sensitivity, which is the photoelectric current from the photocathode, divided by the incident radiant power at a given wavelength. At a certain wavelength, the quantum efficiency, the number of photoelectrons emitted from the photocathode divided by the number of incident photons, is proportional to radiant sensitivity.

Several layers of high permeability magnetic shielding, including a one layer Photonis MS172 shield, surround the PMTs. A $\sim 2 \mathrm{~cm}$ wide white teflon coupler is added between the Alzak pipe and the PMT to protect the PMT against possible sparks between the PMT cathode and the aluminum light pipe. The coupler, PMT and its voltage divider are held in a black PVC (Polyvinyl Chloride) pipe at the end of the light guide. 


\subsubsection{Detector Housing and Mechanics}

The quartz radiator elements, the aluminum radiator holders, and the cone light guides together are enclosed in an aluminum housing (radiator box) to provide a shielded environment from ambient light in the experimental hall (see Fig. 3.22). The geometrical center of the radiator elements is the center of the box. To minimize scattering effects, thin front and back aluminum windows of thickness $0.6 \mathrm{~mm}$ were used.

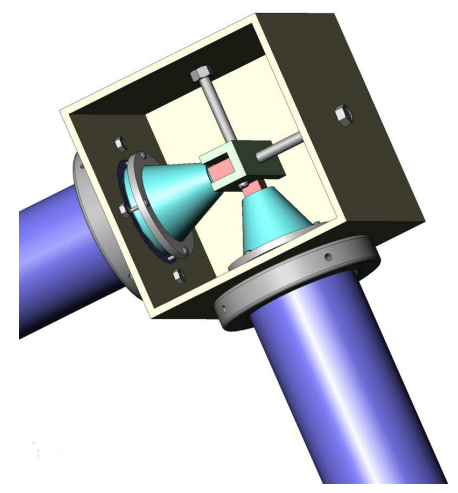

(a)

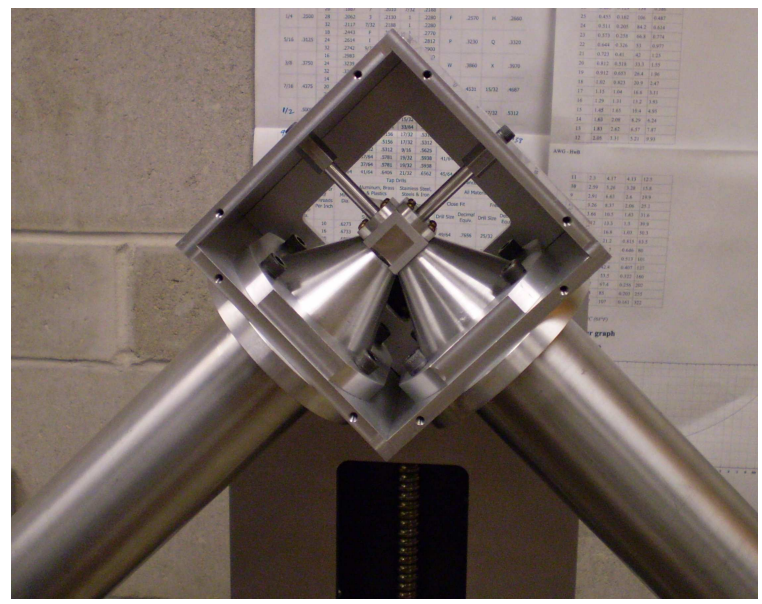

(b)

Figure 3.22: (a) A 3D CAD model for the scanner detector housing. (b) A view of the inner structure of the as-built scanner detector housing. The front window and back window of the radiator box were removed so that the radiator elements, radiator holders and the cone light guides can be seen.

\subsection{Linear Motion System}

\subsubsection{Hardware}

The 2D linear motion assembly consists of two stainless steel ball-screw-driven tables - one mounted on the other orthogonally, giving the detector the ability to move in both $x$ - and $y$-directions in Qweak coordinates. Fig. A.1 in the appendices shows an engineering drawing of the 2D linear motion assembly from Lintech-Motion [104]. 
A custom built 19-inch (48.3 cm wide) rack-mount control box (see Fig. 3.23 (a)) was designed to house several motion control components. Inside the box, two S300 servo-amplifiers by Danaher Motion [105], a DMC-4020 motion controller by Galil Motion Control [106], a 24 V DC power supply, and power on/off switches are assembled to form a PID (proportional-integral-derivative) control loop. Commands are sent by the Galil card, communicating the required analog speed to the servo-amplifiers. The amplifiers then provide the required drive current needed by the servomotors to drive table via the ball screw. The position information produced by a resolver (a rotary transformer) in the motor is encoded in the servo-amplifiers, and sent back to the Galil card. The communication between the Galil card and the host PC in the Q-weak electronics cage is performed through Ethernet.

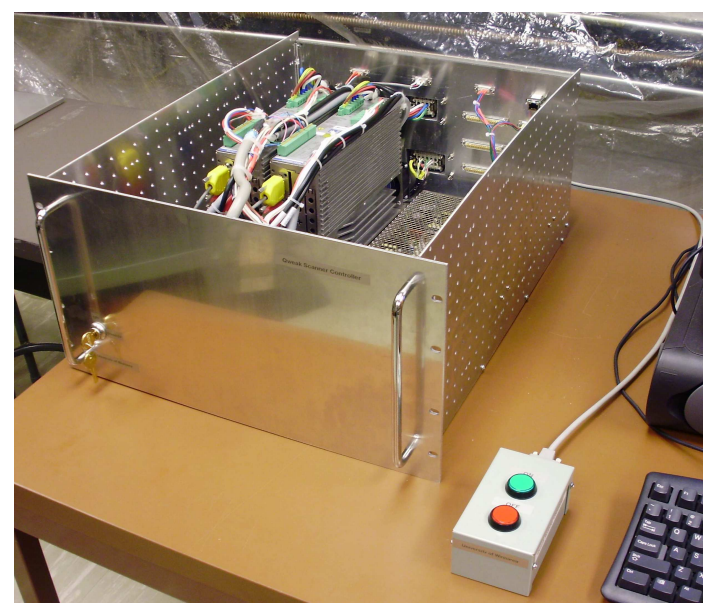

(a) The 19-inch control box for the scanner 2D linear motion system

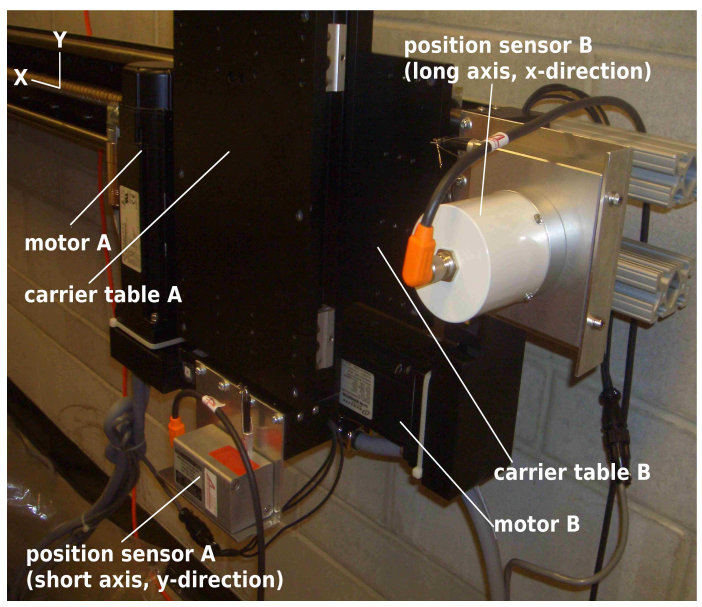

(b) Two position sensors attached on $2 \mathrm{D}$ tables

Figure 3.23: Pictures of the motion control and position recording hardware.

The control box was shielded in a $\mathrm{Pb}$ and concrete housing and placed downstream of the scanner, close to the 2D-motion assembly. Radiation hardness of this system is a concern. Thus far, the system has proven to be more robust against radiation damage than the Ethernet repeaters used to communicate with it in the experimental hall. A dedicated Ethernet line was also created to minimize communication errors (see Section 3.10.4). 
The maximum motion ranges for $x$ and $y$ tables are $2.5 \mathrm{~m}$ and $0.35 \mathrm{~m}$, respectively. These are large enough to scan over the entire fiducial area of the main detector and beyond (see Section 3.10.3). When positioned upstream of the main detector, the scanner can perform a full bar scan, covering $2 \mathrm{~m} \times 0.25 \mathrm{~m}$ area. When downstream of the main detector, the motion is restricted to a central scan over $1 \mathrm{~m} \times 0.25 \mathrm{~m}$ range due to spatial constraints arising from conflicts with the PMT housings of the main detector. For each table, there are two end-of-travel (EOT) switches mounted on each end to limit the motion range, and one home $(\mathrm{H})$ switch to define an absolute reference position. Position along the table is determined relative to the home switch. For added safety, a pair of mechanical fail-safe switches was added to the ends of each table in case the EOT switches failed to function. These devices are expected to work in a radiation harsh environment for a long period of time, so the originally equipped semiconductor proximity sensors, which are used as EOT and home switches, were also replaced with more reliable mechanical switches (Honeywell [107] 11SM1 with a solder pin and JS-5 roller leaf actuator). Furthermore, an emergency stop mechanism designed in the control circuit will halt the motion in response to any switch failure, to avoid collisions with the surrounding objects.

Two position sensors, linear displacement draw wire potentiometers by A-Tech Instruments [108], are installed on the tables (see Fig. 3.23 (b)) to measure instantaneous detector positions. The use of potentiometer-based position sensors provides a convenient way to synchronize the scanner output rate with position signals in the Q-weak fast DAQ system. They can be read out in an ADC synchronously with each scaler read. Due to concerns of radiation hardness, these position sensors are configured as pure resistive potentiometers without any other signal transducer inside. They are attached to one end of the $x$ and $y$ axes, and their stainless steel cables are hooked on the moving $x$ and $y$ carrier boards. When a regulated stable voltage is applied across the potentiometer, the output voltages, which are proportional to the length of cable drawn, directly provide instantaneous position information. 


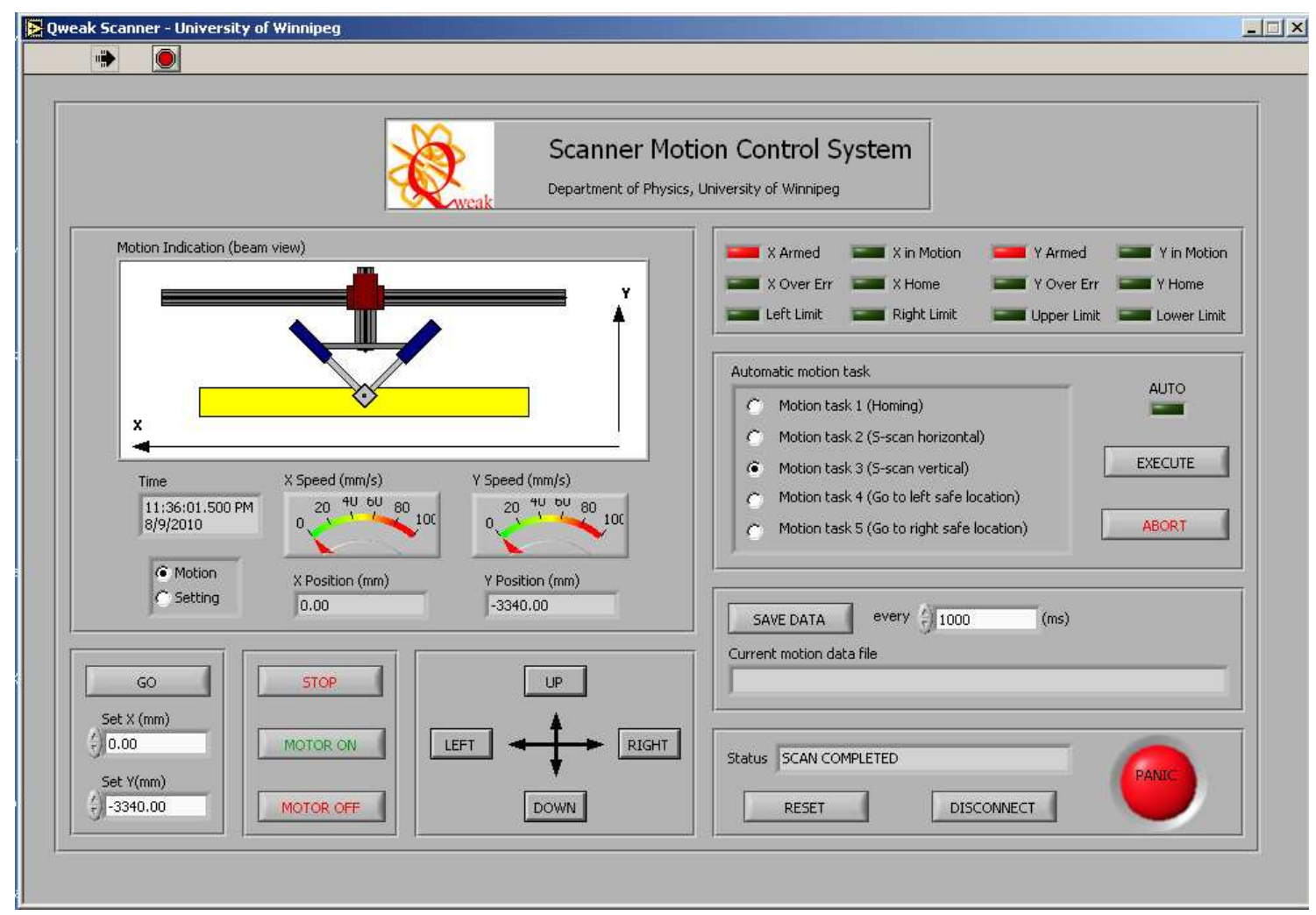

Figure 3.24: The scanner 2D motion control GUI indicates the real-time position information and the status of each switch.

\subsubsection{Software and GUI}

For operational convenience, a graphical user interface (GUI) was developed with LabView (Laboratory Virtual Instrumentation Engineering Workbench), a graphical development environment provided by National Instruments [109]. Several Dynamic Motion Control (DMC) scripts were coded to conduct different motion tasks for various scan patterns. With this GUI, real-time motion status and position information can be displayed. The scanner control PC is located in the Q-weak electronics cage and may be accessed either physically or from the Linux machines in the counting room for convenient control and monitoring. 


\subsubsection{Mounting of Detector on the 2D Motion System}

The scanner detector is mounted on its $2 \mathrm{D}$ motion system via a $39.4 \mathrm{~cm} \times 20.3 \mathrm{~cm} \times 1.0 \mathrm{~cm}$ bridge plate, which was machined to connect the 2D carrier board on the one end, and the detector back supporting plate on the other end. Since at different $z$-planes, the image of the elastic scattered electron locus shifts in radius (in the global $y$-direction), the distance between detector and the bridge plate must be adjusted correspondingly. The bridge plate was therefore implemented with multiple mounting holes, making it easy to adjust the detector mounting position in the radial direction. So far, scanner locations in two z-planes have been implemented. From a JLab survey report [110], one location is $13.92 \mathrm{~cm}$ downstream of the central $z$ of the main detector, and the other is $18.48 \mathrm{~cm}$ upstream. To reach the upstream location, a $22.86 \mathrm{~cm}$ long (in z) extension stage is added between the detector and the 2D linear-motion table (see Fig. 3.25 (a)). The scan range in each plane is listed in Table 3.3. Technical drawings for the 2D motion system, detector and all mounting parts are available in Ref. [111]. An additional z-position further downstream is being discussed for Q-weak Run II, and another extension bracket has been designed at JLab (see Fig. 3.25 (b)). The goal of this additional $z$-position is to study pre-radiator effects with a broader scan range in the $x$-direction downstream of the main detector element, which is currently limited to a half-bar scan by the PMT housings of the main detector.

\subsubsection{Mounting in Q-weak}

The long table of the scanner 2D motion system is bolted to a stainless steel U-channel, which serves as a backbone to avoid distortion of the long, heavy ball-screw assembly. The U-channel is in turn mounted on the Region 3 rotator legs. Unlike other tracking detectors, which may be rotated to other octants, the scanner is fixed in the bottom octant (octant 7). This scanner mounting design, despite its limitation to one octant, was selected for simplicity, with an option to pursue mounting in other octants if confusing trends in comparisons between tracking and main detector parity-mode data arose. This additional option has not yet been pursued.

After installation, the detector was fiducialized and surveyed by the JLab survey group using 


\begin{tabular}{l|cccc}
\hline \hline & $\begin{array}{c}\text { Z-location } \\
{[\mathrm{cm}]}\end{array}$ & $\begin{array}{c}\text { X-range } \\
{[\mathrm{cm}]}\end{array}$ & $\begin{array}{c}\text { Y-range } \\
{[\mathrm{cm}]}\end{array}$ & $\begin{array}{c}\text { Elastic peak in Y } \\
{[\mathrm{cm}]}\end{array}$ \\
\hline \hline MD octant 7 & 577.875 & {$[-100.00,+100.00]$} & {$[-344.00,-326.00]$} & -331.70 \\
\hline Upstream scan & 559.394 & {$[-100.00,+100.00]$} & {$[-339.00,-314.00]$} & -323.00 \\
Downstream scan & 591.792 & {$[-50.00,+50.00]$} & {$[-352.00,-326.00]$} & -336.70 \\
\hline \hline
\end{tabular}

Table 3.3: Scanner detector's motion ranges upstream and downstream of the main detector in octant 7, in the Q-weak global coordinate system. The location of octant 7 is listed in this table as well for reference. For the downstream location, the scan range in the x-direction is limited by the PMT housings of the main detector, and that in the y-direction is limited by the motion range $(26 \mathrm{~cm})$ of the motion table.

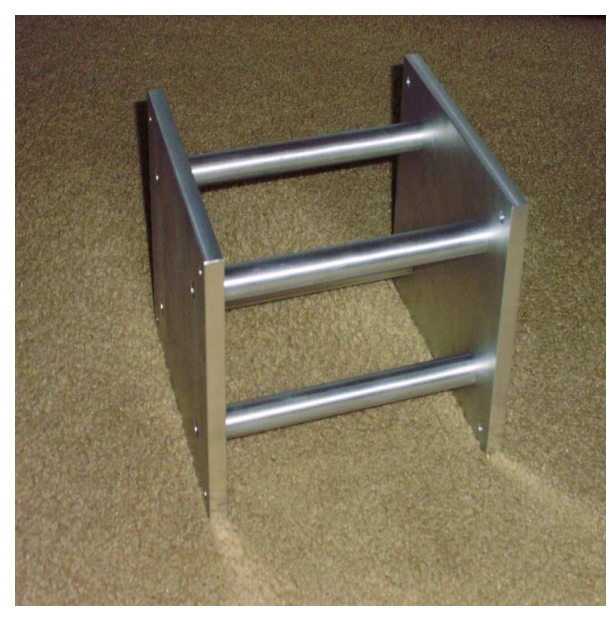

(a) The extension stage built to mount the scanner detector $18.5 \mathrm{~cm}$ upstream of the main detector.

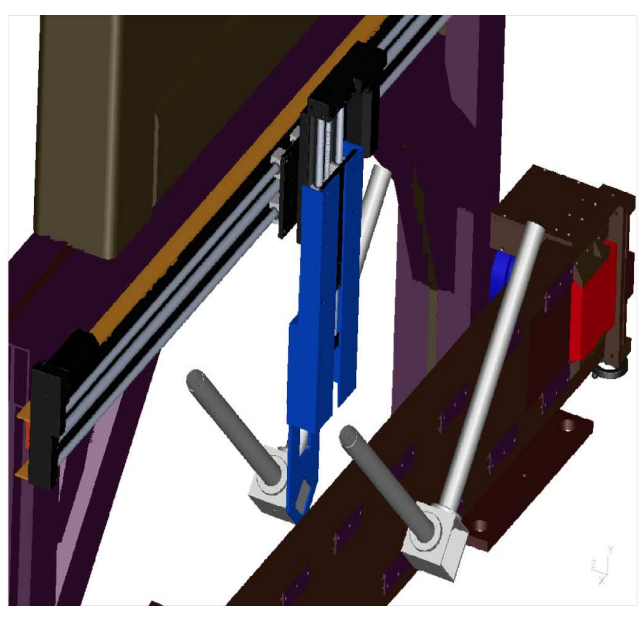

(b) The extension bracket design for scanner mounting at an additional downstream location [112].

Figure 3.25: Extension brackets for the mounting scanner detector on the $2 \mathrm{D}$ motion system at different $z$-planes.

a CMM (coordinate measuring machine). The scanner survey results [110] indicate that it has a position resolution of $\sim 100 \mu \mathrm{m}$ with high reproducibility $(<50 \mu \mathrm{m})$, as expected. The survey also gave a precise measurement of the scanner home position and other calibration parameters. 
Due to limited space and motor cable length, the control box for the motion system was positioned inside the Ferris wheel main detector support structure, $\sim 1 \mathrm{~m}$ behind the scanner detector. It was shielded inside a Pb brick and concrete structure located closer to the beam pipe. All signal cables from the detector and motion controller were routed to the Q-weak electronics cage through cable trays and patch panels. Shielded cables (RG-58 coaxial cable) are used for the signals, and they are isolated from each other and from other subsystems to reduce noise pick-up and cross-talk.

The scanner control PC is located in the Q-weak electronics cage. Communication between it and the Galil control card in the experimental hall was found to suffer from heavy network traffic during commissioning. Two methods were used to resolve the communication problem. One was to put the scanner communication onto its own dedicated Ethernet network. Another was to implement communication with the scanner controller via its serial RS-232 port through the Hall C port server, as an alternate means of communication in the event of a failure of Ethernet-based communications. The communication now performs reliably via both mechanisms.

\subsection{Electronics and DAQ}

The scanner electronics were integrated into the Q-weak electronics and DAQ system. Since the scanner detector must operate in both calibration mode and parity mode, the corresponding electronics had to accommodate the two different functions. As shown in Fig. 3.26, the scanner detector uses a set of NIM and VME modules to record detector PMT outputs and position sensor outputs.

In Q-weak's tracking mode (“calibration mode”), each scanner PMT's analog pulses are digitized by a CAEN V792 12-bit QDC. A JLab F1 TDC is used to measure the timing in relation to other detectors. Four channels of a Struck Innovative System SIS3801 scaler count the two PMTs' singles rates, the coincidence rate and the accidental coincidence rate (the latter is defined by delaying one of the PMT signals by $100 \mathrm{~ns}$ with respect to the other). Position signals are digitized by both a CAEN V792 QDC and a TRIUMF VQWK 18-bit ADC. In calibration mode, the QDC is triggered by several different sources, including the trigger scintillator, the main detector, and the 


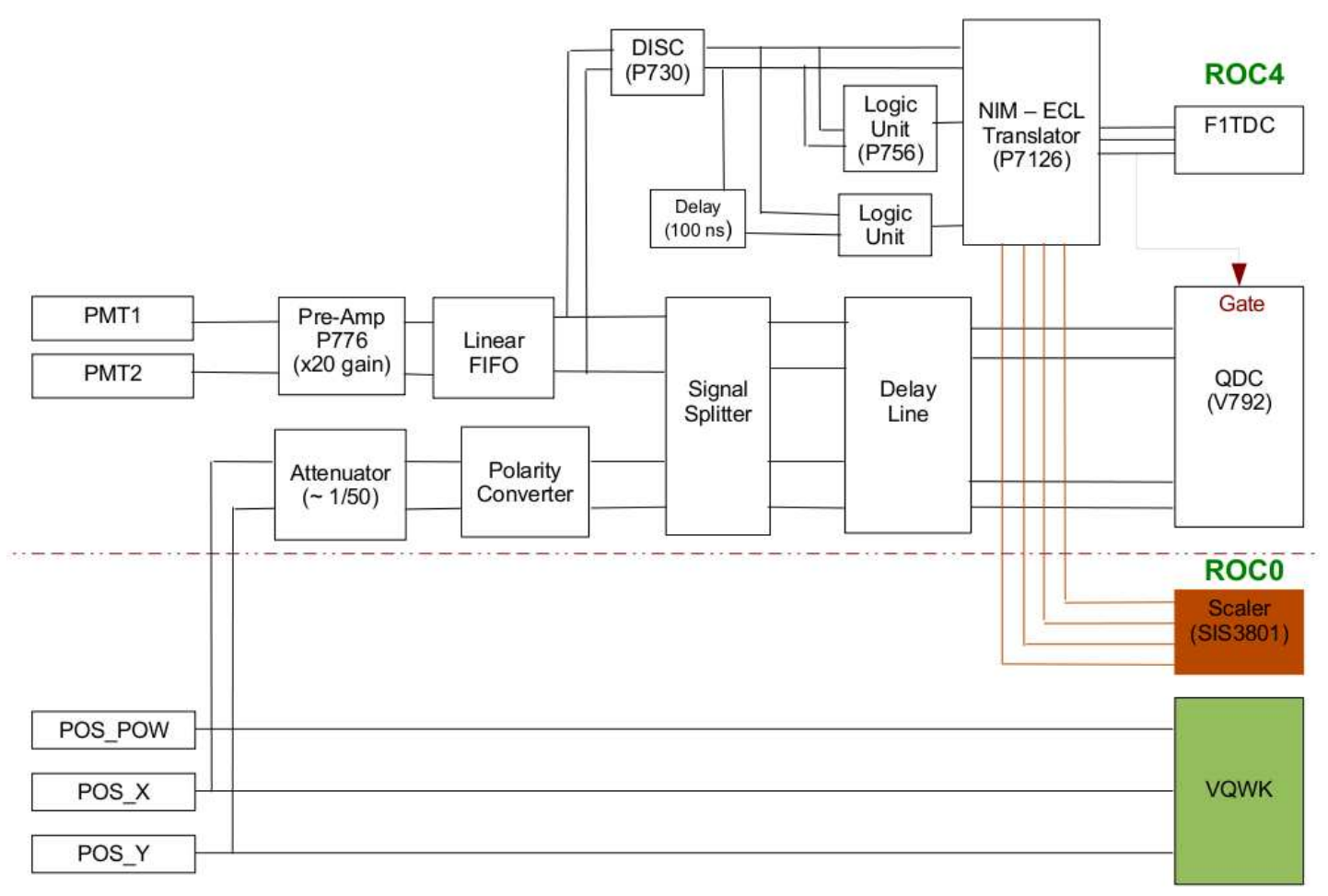

Figure 3.26: Block diagram of the scanner electronics chain (discussed in the text).

scanner self-trigger. In parity mode, the MPS trigger is used to generate reads of the four scaler channels and the detector position, which are recorded by the VQWK ADC.

Scanner position measurements are expected to have $100 \mu \mathrm{m}$ resolution at the nominal $\sim 5 \mathrm{~cm} / \mathrm{s}$ motion speed; to read these to sufficient accuracy requires at least 14-bit ADCs with less than $2 \mathrm{~ms}$ AD converting time for the $2 \mathrm{~m}$ long motion range. The 18-bit VQWK integrating ADC meets these requirements. The V792 12-bit QDC is used as a backup. In order to match the signal level and impedance for these two types of ADCs, voltage followers were constructed for the VQWK ADCs and $\times 1 / 50$ attenuators were used for the V792 position channels.

For rate measurements, in addition to recording the coincidence from both PMTs, a second delay line was set up in the circuit to record out-of-time coincidences as a direct measurement of 
accidental coincidences. As will be discussed in Section 5.1.1, the scanner PMTs at times sense significant backgrounds, leading to high singles rates, and thus high accidental coincidence rates, which may contribute a large fraction of the total coincidence signals. In order to obtain the true coincidence rate arising from electrons striking the quartz radiators, the measured coincidence rate must therefore be corrected. Details of the correction procedure will be discussed in Chapter 5 .

\subsection{Installation and Commissioning}

The scanner system was designed, built and tested at the University of Winnipeg before being shipped to JLab at the end of 2009. It was installed in the experimental hall in the summer of 2010, followed by detector survey and alignment procedures. As seen in Fig. 3.27, the detector can be installed either at upstream or downstream of the main detector in octant 7 to make measurements in two different $z$-planes.

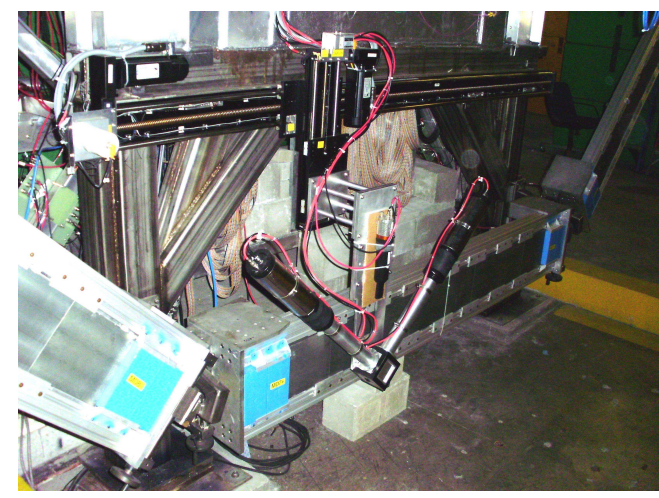

(a)

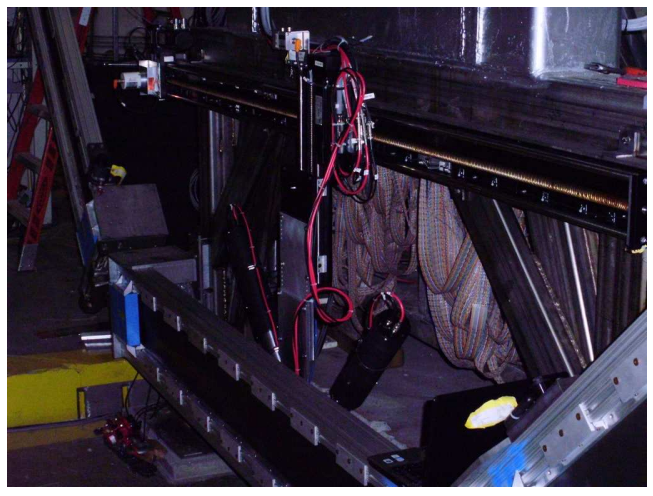

(b)

Figure 3.27: (a) The scanner installed in front of the main detector in the bottom octant. (b) The scanner installed behind the main detector.

During the Q-weak commissioning phase in fall 2010, the scanner detector, motion system, electronics and DAQ were tested, including detector light tightness tests, dark rate measurements, signal timing match, discriminator threshold and pulse width optimization. The scanner system was successfully commissioned, and it was used to perform measurements at various beam currents with 
different Q-weak targets.

The scanner was the first detector to produce an image of electrons reaching the focal plane, which gave early confirmation that the Q-weak spectrometer was functioning properly. Soon after the first scans were acquired and displayed online, the device was heralded as "having paid for itself already" [113]. Fig. 3.29 shows an example rate map acquired by the scanner detector at $50 \mu \mathrm{A}$ beam current with the $\mathrm{Q}$-weak $\mathrm{LH}_{2}$ target during Q-weak commissioning. The rate map was made from the data acquired with a vertical S-shape scan pattern (see Fig. 3.28). During data taking, the detector was moved continuously in the $y$-direction at various fixed $x$-positions, while measuring the instantaneous rate and position $\left(R_{i j}, x_{i}, y_{j}(j=1-26)\right.$, where $i$ and $j$ represent the bin numbers in $x$ and $y$ ). The position in the $x$-direction changes in $1 \mathrm{~cm}$ steps (also used as the bin size), covering a total of 200 bins. The average rate within each bin, and the corresponding average position fill a 2D histogram to form the measured rate map. The one shown in Fig. 3.29 is a rate map after accidental coincidence subtraction from the raw coincidence rate distribution.

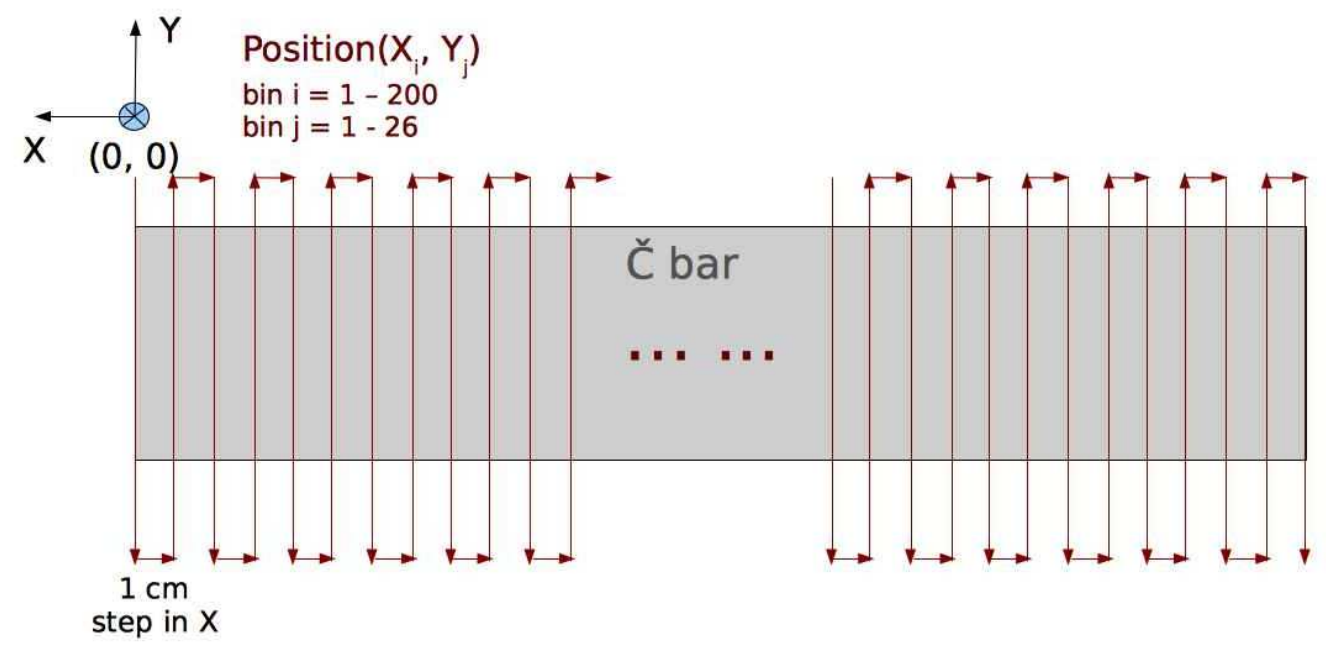

Figure 3.28: Schematic diagram of the scanner's vertical S-scan pattern. The system starts the motion from the home positions for both axes, and moves following the path indicated by the arrows. The detector moves continuously in the $y$-direction with $1 \mathrm{~cm}$ steps in the $x$-direction. 


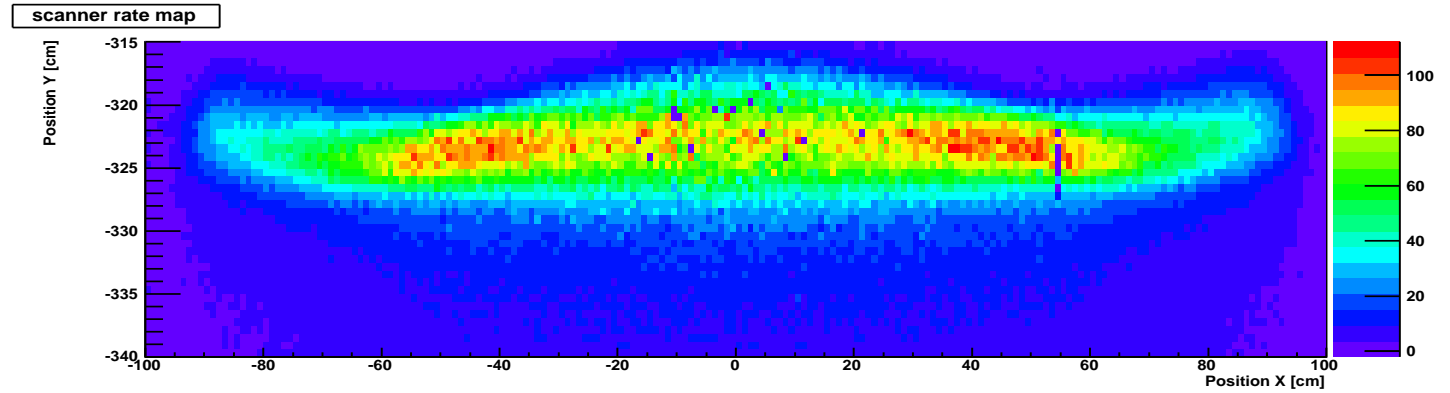

Figure 3.29: The measured rate distribution with accidental coincidence rates subtracted, at $50 \mu \mathrm{A}$ beam current with $\mathrm{LH}_{2}$ target (run 6616). In this figure, the $x$ - and $y$-coordinates are the Q-weak global coordinates in units of $\mathrm{cm}$. The intensity scale shows the rate in units of $\mathrm{kHz}$. The rate map is not normalized to beam current, but a beam current cut was applied in the analysis, and a $0.24 \mu \mathrm{A}$ RMS width of beam current distribution was found during this scan period. A vertical strip near $x=54 \mathrm{~cm}$ due to a beam trip can also be observed.

\subsection{Monte Carlo Simulations of Scanner Applications}

Monte Carlo simulations were used to study various scanner applications and motion pattern optimization to address specific physics questions. For instance, the scanner is sensitive to percent-level QTOR magnetic field scale variations, especially along the mid-plane of the Čerenkov bar. Fig. 3.30 shows a simulation of the elastic electron rate along the bar center as a function of the value of BFIL, a factor used in the Monte Carlo simulation to express the strength of the QTOR magnetic field. A value of the QTOR DC current of $8615 \mathrm{~A}$ at a coil temperature of $20^{\circ} \mathrm{C}$ corresponds to a BFIL factor of 1. For a BFIL factor of 1.04, for example, the corresponding QTOR current is $8960 \mathrm{~A}$. The scanner can thus be used to monitor spectrometer optics and verify the beam locus on the main detector during parity runs by performing a vertical central strip scan, which can be done within one minute. Possible variations of the QTOR magnetic field may affect the rate in detector acceptance, thus the $\left\langle Q^{2}\right\rangle$. The overall main detector rate itself would be relatively insensitive to these small changes. The scanner detector possesses the ability to make quick, minimally invasive measurements of the vertical rate profile, and hence can be used to monitor the variation of $\left\langle Q^{2}\right\rangle$ across production runs.

As seen in Fig. 3.31, simulation studies indicate that the scanner has an approximately linear 
response to magnetic field variations and its sensitivity to BFIL ( $\triangle \mathrm{X} / \triangle \mathrm{BFIL})$ is about $1.2 \mathrm{~cm} / 1 \%$. This has now been verified by beam test data, as will be discussed in Chapter 5 .

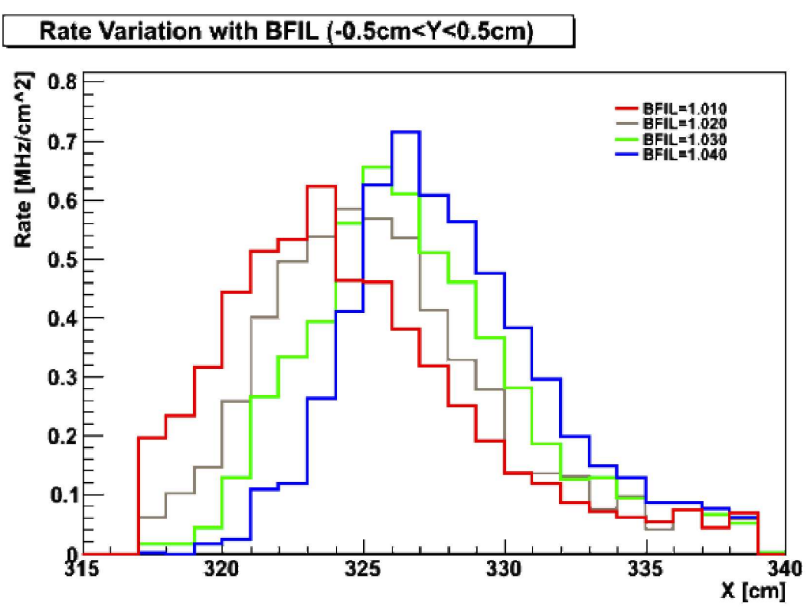

Figure 3.30: Simulated rate distribution with varying BFIL. The position of the electron peak along the bar center is sensitive to BFIL variations. With an increase of magnetic field, the elastic peak moves to outer radii in the spectrometer.

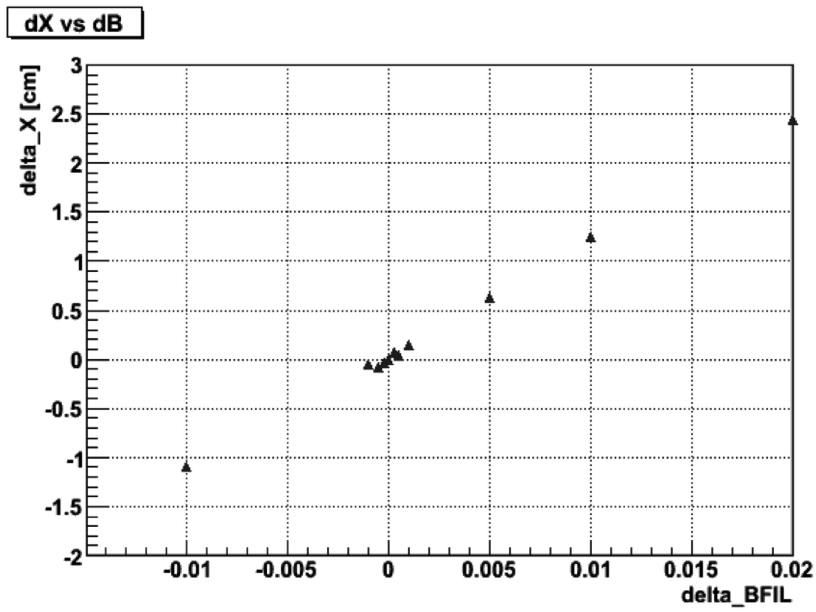

Figure 3.31: Simulated vertical position variations of the elastic rate distribution centroid determined by the scanner versus BFIL variations relative to the nominal value of $\mathrm{BFIL}=1.04$. 


\section{Chapter 4}

\section{Momentum Transfer Determination}

The light-weighted average momentum transfer squared $\left\langle Q^{2}\right\rangle$ for elastically scattered electrons must be determined to $<1 \%$. This is achieved from auxiliary measurements with a set of high resolution tracking detectors at low beam current $(<100 \mathrm{pA})$ in event mode runs. This necessitates dedicated tracking software to reconstruct individual tracks according to the output hit information from tracking chambers. This chapter will be focused on the Q-weak Track Reconstruction (QTR) program one of the areas in which I made substantial contributions to Q-weak. Preliminary tracking results analyzed with QTR on an analyzable subset of the tracking data will be presented.

\subsection{Overview}

As introduced in Chapter 2, the tracking system includes the Region 2 HDCs (located upstream of QTOR) and the Region 3 VDCs (downstream). The tracking detector output signals are pulse arrival times, digitized by TDCs. These signals carry information about which drift cells have been hit, and what the drift distances are, via time-to-distance conversion. The event-based TDC values are recorded and transferred to the back-end processes and stored in CODA format. The tracking analyzer decodes the CODA events and registers them in a hit container, then performs track reconstruction on the hits through QTR. The main functions of QTR are to identify and reconstruct 
individual tracks, based on sets of hits in the container for the three tracking regions.

Each track is expected to provide a set of hits in 12 planes of HDCs, and 4 planes of VDCs, each forming separate straight line partial tracks, since the magnetic field is close to zero at the location of the tracking chambers. The two track segments are related in the software by simulating the passage of an electron through the magnetic field of the QTOR magnet, a procedure known as "swimming". In this way, front partial tracks are matched with back partial tracks to reconstruct global tracks (see Fig. 4.10 and Fig. 4.14 for reference). For an electron trajectory, the scattering vertex and scattering angle are reconstructed from the upstream chambers, and the momentum for the elastic event is confirmed by the downstream chambers. The $Q^{2}$ for each track is then calculated based on the scattering angle and momentum before and after scattering, using Eqn. 1.20. Finally, in order to determine the light-weighted $\left\langle Q^{2}\right\rangle$, the main detector light response is correlated with each track. In general, the track reconstruction procedures in QTR could be summarized as:

- Establish partial tracks for front and back segments, respectively, by using a "treesearch" pattern recognition algorithm (see Section 4.3);

- Combine front and back partial tracks into global trajectories in space with a "bridging" routine where the swimming procedure mentioned above is iterated to find the best track momentum (see Section 4.4);

- Determine $Q^{2}$ with reconstructed global track parameters - scattering vertex, scattering angle and momentum;

- Map out the main detector light response to elastic events and calculate the light-weighted $\left\langle Q^{2}\right\rangle$.

From wire hits to reconstructed tracks, two major algorithms, tree-search and bridging, are used in QTR. Since these processes are time critical, special care was taken to optimize the algorithms and code structures for speed of execution. In the following sections, the main components of QTR will be discussed in detail. 


\subsection{Coordinate Systems and Naming Conventions}

For the convenience of further discussion, it is worthwhile to introduce the Q-weak coordinate systems and naming conventions. The QTR program, together with the Q-weak simulation, follows the same definitions and conventions as those used in the experiment installation.

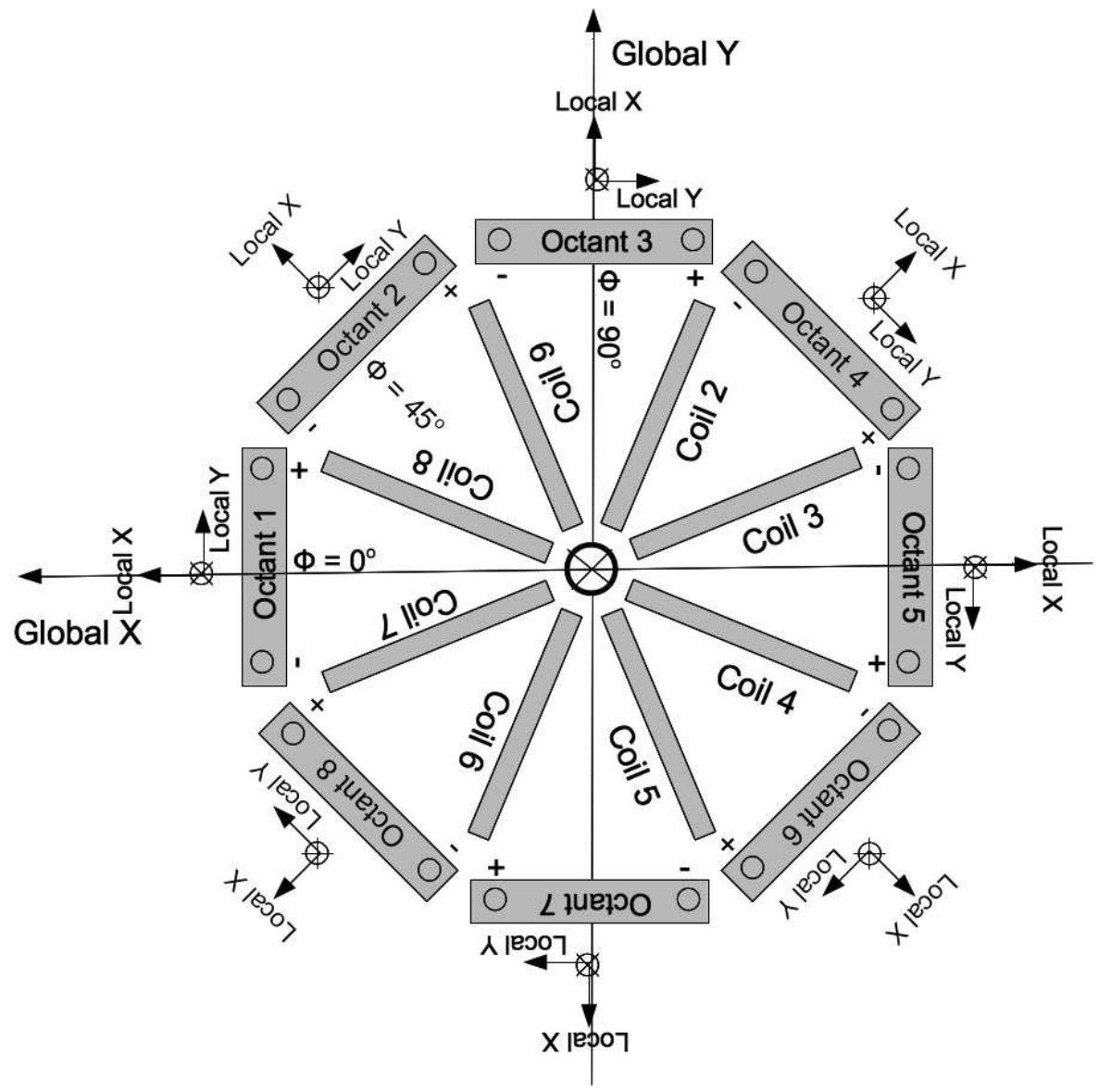

Figure 4.1: The Q-weak coordinate systems, including a global coordinate system and local coordinate systems.

The geometrical center of the QTOR spectrometer is the origin of the Q-weak coordinate system. The global $x$-axis points horizontally to beam left, the $y$-axis points vertically upward, and the $z$-axis points downstream along the beam axis to form a right-handed Cartesian coordinate system. Eight magnet coils, numbered from 2 to 9 in the clockwise direction when looking downstream (see 
Fig. 4.1), are positioned symmetrically around the beam axis. They separate the experimental space into eight octants, numbered from 1 to 8 in the clockwise direction, with octant 1 starting at the azimuthal angle $\phi=0^{\circ}$, with each of the other octants being rotated about the $z$-axis by $45^{\circ}$ relative to the last, in turn. The azimuthal angle $\phi$ is defined in the corresponding cylindrical coordinates with respect to the global $x$-axis mentioned above.

In addition to the global coordinate system, there are local coordinate systems defined for each of the eight octants. The local $x$-axis points radially outward from the beam line; the local $y$-axis points in the direction of increasing $\phi$, and the local $z$-axis is the same as the global $z$-axis. Therefore, in octant 1 , the local $x, y, z$ directions are consistent with the global $x, y, z$ directions. In a few cases, the origin of a local coordinate system is assigned at a specified position of the detector in that octant.

For the main detector in each octant, the two PMTs at the ends of the quartz bar are marked as "+" and "-", respectively, according to the PMT"s $y$-location in its local coordinate system. In the Q-weak parity or tracking analyzer, each main detector PMT is identified with a specific name that reflects its local and global position, e.g., md1p (main detector, octant $1,+$ ), md2m (main detector, octant $2,-)$, and so on.

For the rotatable tracking drift chambers (the HDCs and VDCs), in order to easily identify which wires have been hit in the planes with different wire orientations ( $x-, u-, v$-wires), an $x u v$ coordinate system is defined in the local coordinates. The HDCs and VDCs have different wire orientations, and thus different $x u v$-coordinate systems, which are shown in Fig. 4.2 and Fig. 4.3, respectively. The HDCs have 12 wire planes per octant with three types of wire strung at constant $x, u, v$ orientations, and the $x$-, $u$-, $v$ - axes in their $x u v$-coordinate system are perpendicular to the corresponding wires [114]. The $u$-axis and $v$-axis point in directions at angles $\theta= \pm 26.6^{\circ}$ with respect to the $x$-axis. The VDCs have 4 planes per octant with two types of wire orientations $(u$-and $v$-wires). In the VDC $x u v$-coordinate system, the $\theta$ angle between $u$-axis (or $v$-axis) and the $x$-axis is $53.13^{\circ}$. 

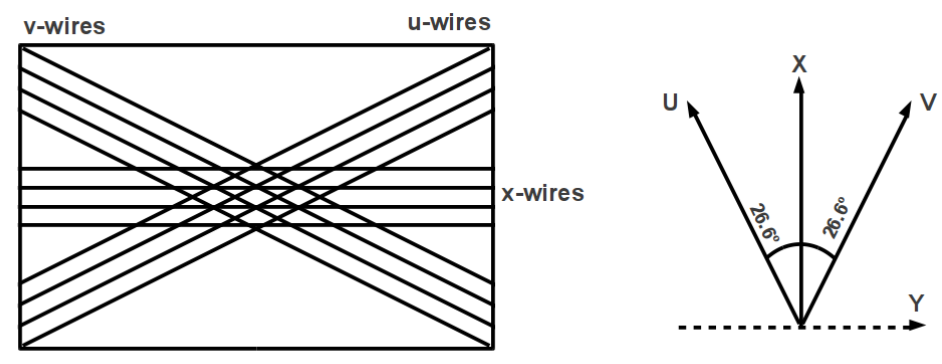

wire directions

X-U-V coordinates

Figure 4.2: The Region $2 x u v$-coordinates (drawings are based on the descriptions in Ref. [114] ). The orientation angles are $\theta_{u}=-26.6^{\circ}$ and $\theta_{v}=26.6^{\circ}$.

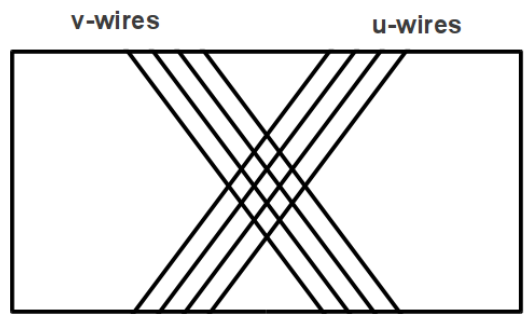

wire directions

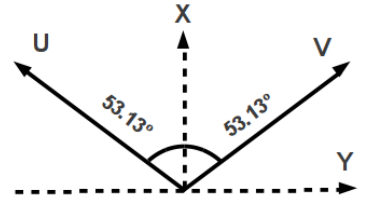

U-V coordinates

Figure 4.3: The Region $3 u v$-coordinates (drawings are based on the descriptions in Ref. [114] ). The orientation angles are $\theta_{u}=-53.13^{\circ}$ and $\theta_{v}=53.13^{\circ}$.

\subsection{Partial Track Finding}

The tracks individually reconstructed by the HDCs and the VDCs are referred to as partial tracks. The partial tracks in the tracking chambers are assumed to be straight lines. Pattern recognition is used to speed the partial track finding process.

\subsubsection{Pattern Recognition Algorithm}

The QTR borrowed an idea from the HERMES Reconstruction Code [115] using "treesearch" as its pattern recognition algorithm. Straight lines are identified via patterns to find tracks in detector systems efficiently. The treesearch algorithm uses a pattern database that contains all possible particle tracks for a given detector system and resolution. 


\section{Bit Pattern}

In order to find straight line partial tracks, the recorded hits from each tracking detector are encoded into a bit pattern. Fig. 4.4 shows a side view of electron partial tracks passing through the HDCs and VDCs. The two types of chambers have different drift directions and chamber orientations with respect to the incident electron path.

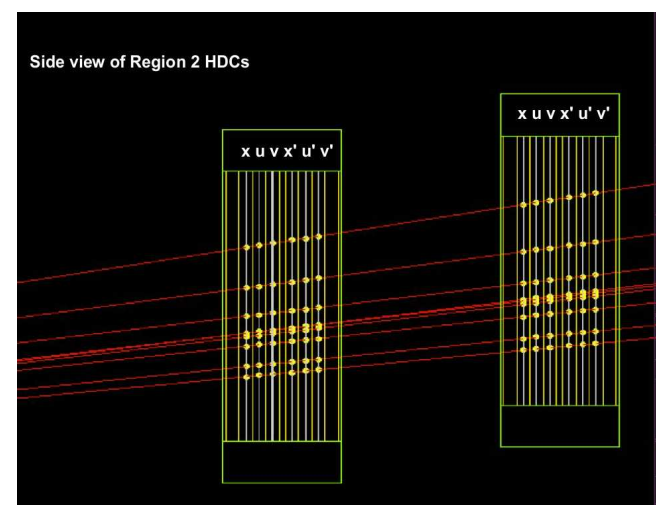

(a) side view of HDC wire planes

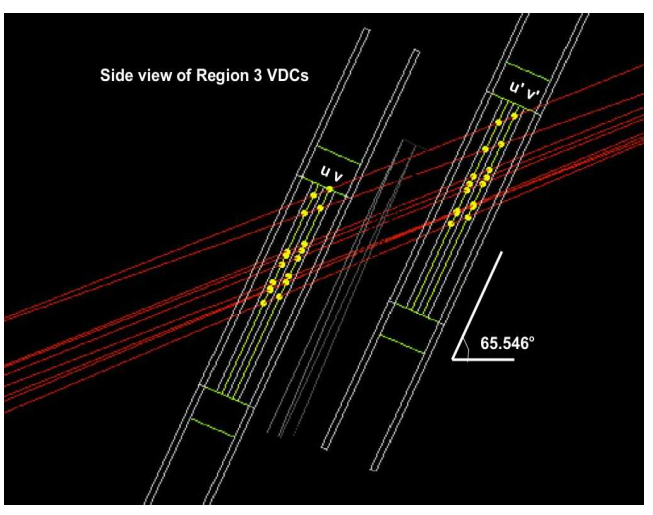

(b) side view of VDC wire planes

Figure 4.4: Side views of the Region 2 and Region 3 wire planes in the Q-weak Geant4 simulation. Note that the VDCs are rotated about their local $y$-axes by $24.454^{\circ}$ for better track resolutions. In both figures, the downstream direction is on the right hand side.

In the HDCs in one octant, there are a total of twelve wire planes with four planes of the same wire orientation in either $u, v$ or $x$. In the HDCs, a nominal particle track is approximately perpendicular to the wire planes (see Fig. 4.5). Each plane is divided logically into $2^{N}(N=1,2,3, \cdots)$ small cells. The main reason for this division is that each cell can be considered as a bit of a binary number to reduce storage and computation time. The cells struck by the incident electron under consideration are registered as "1" (or "on"), and others as "0" (or "off"). This forms a 1D bit pattern for a single wire plane. For any "on" bit, only the drift distance can be determined, as opposed to the actual track position, since there is an intrinsic left-right ambiguity. Information from consecutive wire planes with the same wire orientation can help to reduce the left-right ambiguity. The combination of 1D bit patterns in these planes forms a 2D bit pattern, as seen in Fig. 4.5.

The VDCs are oriented in such a way that a particle with nominal trajectory will go through four 


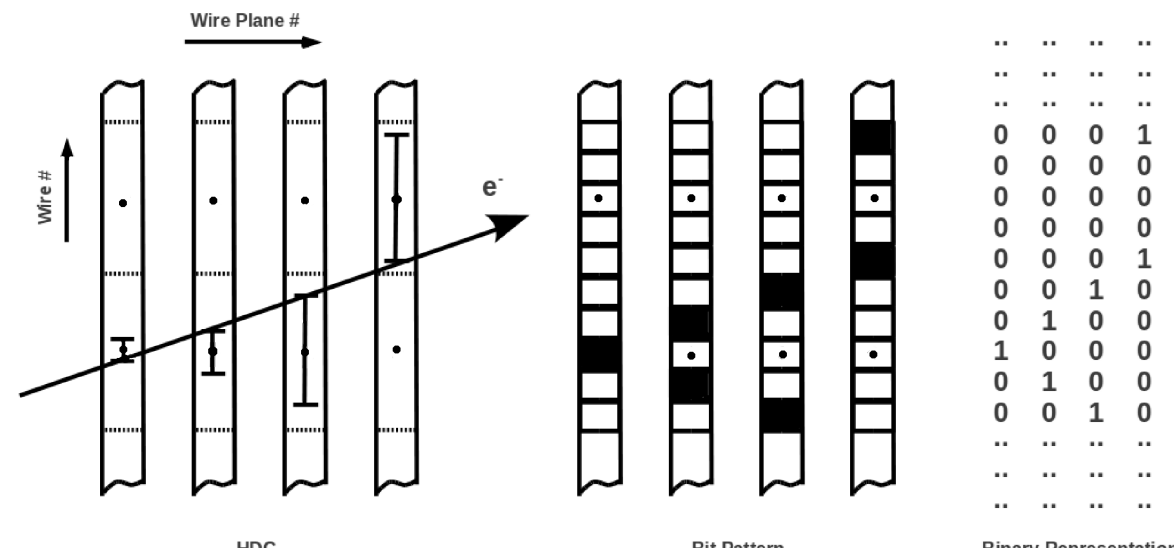

Figure 4.5: Example of Region 2 HDC bit pattern in four wire planes.

to eight drift cells in a single wire plane. Since the electron drift direction is vertical (perpendicular to the plane), each plane of wires has its own 2D bit pattern, as seen in Fig. 4.6. The maximum number of hits for an ideal track is eight, so the bit pattern has eight columns.

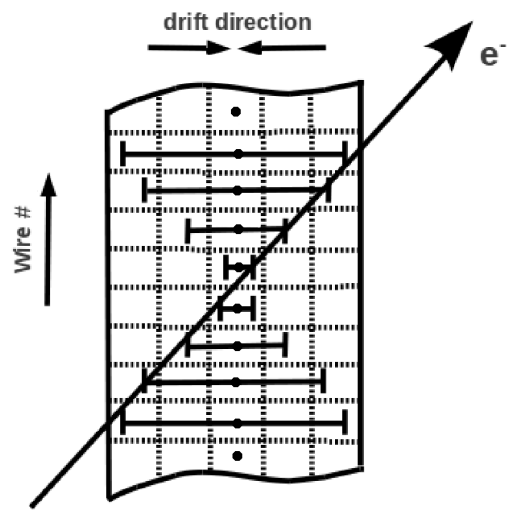

VDC

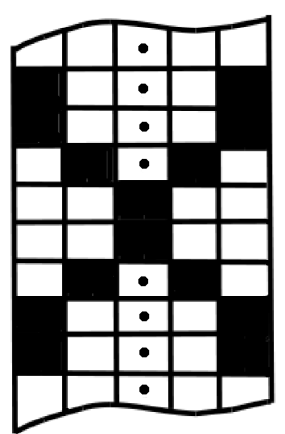

Bit Pattern

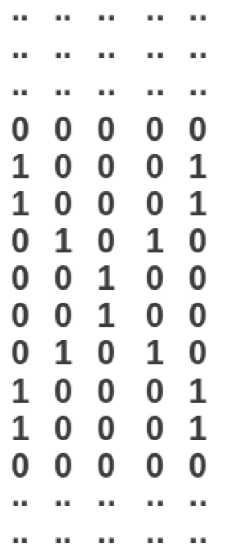

..

Binary Representation

Figure 4.6: Example of Region 3 VDC bit pattern in a single wire plane.

A pattern database is generated, which contains all potential bit patterns. By matching detector encoded patterns against the pre-defined patterns in a database, tracks are identified. In QTR, the 
pattern database is generated starting from a two bit 'parent' pattern, then recursively producing all potential 'child' patterns progressively doubling the resolution at each stage.

\section{Pattern Matching}

Pattern matching is the process of searching the pattern database, and checking if a subset of detector hits is close to the bits in the bit patterns. Matched detector hits are then fit to a straight line as track candidates. When matching the actual detector hit pattern with respect to the patterns in the pattern database, a linear searching method is slow and thus impractical. In QTR, a recursive searching method is used. For example, consider starting with a trivial pattern at a resolution of one bin. All 'child' patterns ('2-bit patterns') at doubled resolution are examined. If a matching pattern is found, the depth of the recursion is increased and the patterns are compared at the new, doubled resolution. Then the search restarts with the '2-bit detector' as the parent pattern. All other child patterns from the original level of resolution are tested, and the track search is repeated at higher resolution. This track search algorithm is highly recursive. The resolution is successively doubled and reaches (chamber width) $\times 2^{-N}$ after $N$ steps. A search of the pattern database finds maximally one track, because only one pattern and one of its children are used for each level of resolution.

\subsubsection{Parameterization of Partial Track Projections}

A set of hits, identified by treesearch to belong to a "good" track, is used to construct the line parameters of a partial track projection in $x, u$, and $v$ planes. The parameters can be expressed in terms of slopes $\left(k_{x}, k_{u}, k_{v}\right)$ and intersections/offsets $\left(b_{x}, b_{u}, b_{v}\right)$ of the linear function

$$
\left(\begin{array}{l}
p_{x} \\
p_{u} \\
p_{v}
\end{array}\right)_{i}=\left(\begin{array}{l}
k_{x} \\
k_{u} \\
k_{v}
\end{array}\right) z_{i}+\left(\begin{array}{c}
b_{x} \\
b_{u} \\
b_{v}
\end{array}\right)
$$

where $\left(p_{x}, p_{u}, p_{v}\right)$ represent the hit positions in terms of the $x, u, v$ coordinates, $z_{i}$ is the $z$ location of the $i$-th $(i=1 \ldots N)$ hit in the detectors along the track. For all the hits in all projections, the linear 
equations can be expressed explicitly as

$$
\left(\begin{array}{ccc}
p_{x, 1} & p_{u, 1} & p_{v, 1} \\
p_{x, 2} & p_{u, 2} & p_{v, 2} \\
\vdots & \vdots & \vdots \\
p_{x, N} & p_{u, N} & p_{v, N}
\end{array}\right)=\left(\begin{array}{cc}
z_{1} & 1 \\
z_{2} & 1 \\
\vdots & \vdots \\
z_{N} & 1
\end{array}\right) \cdot\left(\begin{array}{ccc}
k_{x} & k_{u} & k_{v} \\
b_{x} & b_{u} & b_{v}
\end{array}\right)
$$

where

$$
\alpha=\left(\begin{array}{lll}
k_{x} & k_{u} & k_{v} \\
b_{x} & b_{u} & b_{v}
\end{array}\right)
$$

is the parameter matrix of partial track projections.

In Eqn. 4.2, there are $3 \times 2$ unknowns, which form the 6 elements of the parameter matrix. The known variables for the linear equations are $p_{x u v, i}$ and $z_{i}$, obtained from tracking measurements, detector geometries and survey results. With more equations than unknowns, the linear equations can be solved by using a least-squares method.

The above discussion is for the simplest case, assuming that all measurements for $p_{x, i}, p_{u, i}$ and $p_{v, i}$ are treated with the same level of uncertainty, i.e.,

$$
\sigma_{x, i} \simeq \sigma_{u, i} \simeq \sigma_{v, i} \quad(i=0 \cdots N)
$$

where $\sigma_{x, i}, \sigma_{u, i}$ and $\sigma_{v, i}$ are the uncertainties of measuring the hit position $p_{i}$ in $x, u$ and $v$ directions, respectively, determined by the chamber resolution. If the above condition cannot be satisfied, the measured hit positions should be weighted by their respective uncertainties $\left(\sigma_{x u v, i}\right)$. Because the equations in Eqns. 4.2 are linear, each individual equation can be directly weighted, for instance, as:

$$
\frac{1}{\sigma_{x, i}^{2}} p_{x, i}=\frac{1}{\sigma_{x, i}^{2}}\left(k_{x, i} z_{i}+b_{x, i}\right)=\frac{k_{x, i}}{\sigma_{x, i}^{2}} \cdot z_{i}+\frac{b_{x, i}}{\sigma_{x, i}^{2}} \cdot 1
$$

It is possible that several tracks identified by treesearch are within one 'road' (i.e., the tracks 
occupy the same bit array). These tracks will have different $\chi^{2}$-values, given by [115]:

$$
\chi^{2}=\frac{1}{N-2} \sum_{i=1}^{N} \frac{1}{\sigma_{i}^{2}}\left[p\left(z_{i}\right)-p_{i}\right]^{2}
$$

The final partial track parameters are determined for the track which has the smallest $\chi^{2}$-value, as being the most likely track responsible for the observed hit pattern.

\subsubsection{Partial Tracks in Three Dimensions}

A partial track is a three dimensional object, whose 3D track parameters can be constructed from the parameters of its $x, u, v$ projections. Defining a coordinate transformation matrix $R$ and its inverse matrix $T=R^{-1}$, as:

$$
R=\left(\begin{array}{cc}
\cos \theta_{u} & \sin \theta_{u} \\
\cos \theta_{v} & \sin \theta_{v}
\end{array}\right), \quad T=\frac{1}{\cos \theta_{u} \sin \theta_{v}-\sin \theta_{u} \cos \theta_{v}}\left(\begin{array}{cc}
\sin \theta_{v} & -\sin \theta_{u} \\
-\cos \theta_{v} & \cos \theta_{u}
\end{array}\right),
$$

where $\theta$ is the orientation angle of the $u$-, $v$ - axes with respect to the $x$-axis, the transformation between the local $(x, y)$ coordinates and the projected $(u, v)$ coordinates is given by:

$$
\left(\begin{array}{l}
u \\
v
\end{array}\right)_{p r o j}=R\left(\begin{array}{l}
x \\
y
\end{array}\right),\left(\begin{array}{l}
x \\
y
\end{array}\right)=T\left(\begin{array}{l}
u \\
v
\end{array}\right)_{p r o j} .
$$

Since Region 2 HDCs have wire planes oriented in the $x$ direction, the $x$ coordinate can also be obtained from direct measurements. The value of $x$ can be extracted from a combination of both direct measurement and $u v$ to $x y$ transformation, weighted by their respective uncertainties. A weighted transformation matrix $T^{\prime}$ can be constructed so that

$$
\left(\begin{array}{l}
x \\
y
\end{array}\right)=T^{\prime}\left(\begin{array}{l}
u \\
v \\
x
\end{array}\right)_{p r o j},
$$


where $x_{\text {proj }}$ is the weighted combination of the directly measured $x$ (referred to as $x_{x}$ ) and the $u v \rightarrow x y$ transformed $x$ (referred to as $\left.x_{u v}\right) ; T^{\prime}$ is the weighted inverse transformation matrix defined as [115]:

$$
T^{\prime}=\left(\begin{array}{ccc}
T_{11} \frac{\Delta x_{x}^{2}}{\Delta x_{x}^{2}+\Delta x_{u v}^{2}} & T_{12} \frac{\Delta x_{x}^{2}}{\Delta x_{x}^{2}+\Delta x_{u v}^{2}} & \frac{\Delta x_{u v}^{2}}{\Delta x_{x}^{2}+\Delta x_{u v}^{2}} \\
T_{21} & T_{22} & 0
\end{array}\right),
$$

$\Delta x_{x}$ is the uncertainty of the directly measured $x$, which is the same as the $\sigma_{x}$ in Section 4.3.2, and $\Delta x_{u v}$ is the uncertainty of the $u v \rightarrow x y$ transformed $x$, determined by $\sigma_{u}$ and $\sigma_{v}$ via error propagation in Eqn. 4.8.

\section{Partial Track Parameters}

From the above procedure, a partial track's $x$-, $u$ - and $v$-projections can be obtained. The recombination of $x$-, $u$-, $v$-projections into a 3D partial track is schematically shown in Fig. 4.7. Partial tracks are parameterized in terms of slopes and offsets in the local $(x, y, z)$ coordinates as:

$$
\left(\begin{array}{l}
x \\
y \\
z
\end{array}\right)=\left(\begin{array}{cc}
k_{x} & b_{x} \\
k_{y} & b_{y} \\
1 & 0
\end{array}\right) \cdot\left(\begin{array}{l}
z \\
1
\end{array}\right),
$$

where the slopes $\left(k_{x}, k_{y}\right)$ and offsets $\left(b_{x}, b_{y}\right)$ parameters are given by:

$$
\left(\begin{array}{ll}
k_{x} & b_{x} \\
k_{y} & b_{y}
\end{array}\right)=T^{\prime}\left(\begin{array}{ll}
k_{u} & b_{u} \\
k_{v} & b_{v} \\
k_{x} & b_{x}
\end{array}\right)_{p r o j}
$$

Eqns. 4.11 and 4.12 describe the trajectory of a straight partial track. 


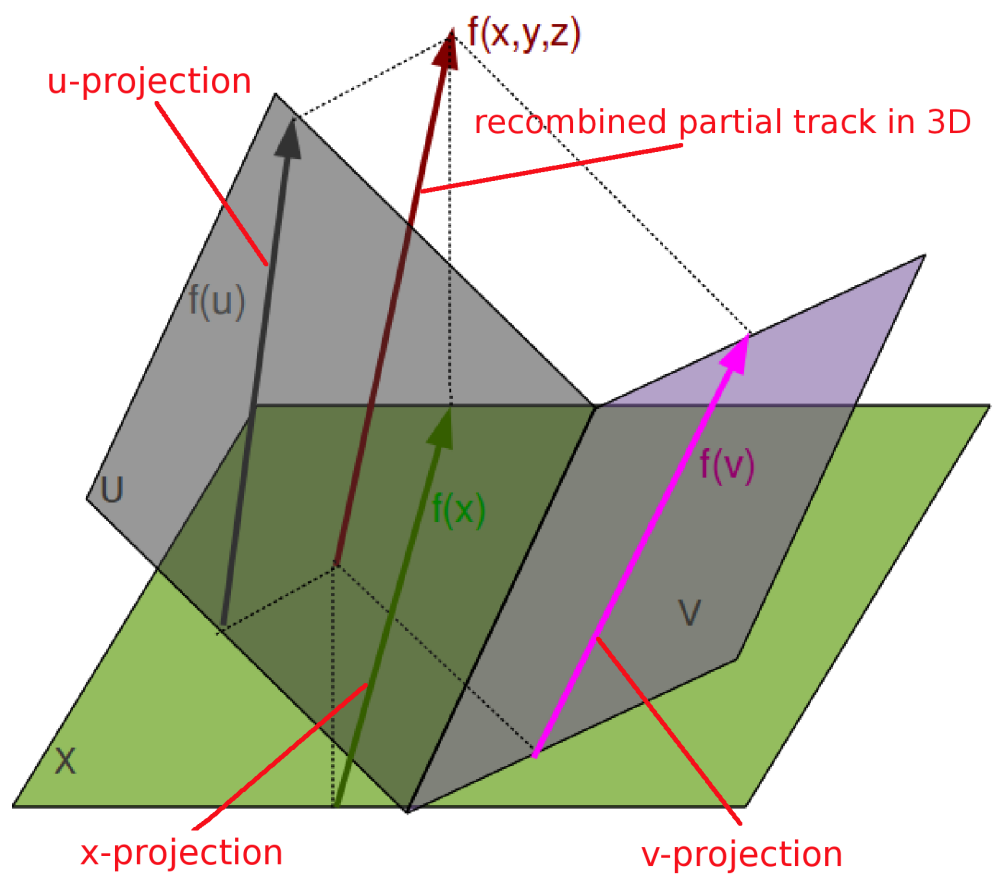

Figure 4.7: Partial track in three dimensions [115], represented by $f(x, y, z)$ in $x-y-z$ coordinates. The upstream partial tracks are reconstructed from the recombination of $u$-, $v$ - and $x$-projections, represented by $f(u), f(v)$ and $f(x)$, from Region 2 and the downstream partial tracks are obtained from the combination of $u$-, v-projections from Region 3. The $u$-, v- and $x$-planes in this figure represent the planes in which the $2 \mathrm{D} u, v$ and $x$ bit patterns are formed.

\subsection{Bridging Partial Tracks}

The partial tracks from the upstream and downstream sections of the spectrometer are combined by "bridging" the tracks through the magnetic field of the spectrometer. A bridging algorithm is used in the QTR code for matching the partial tracks and determining the parameters (momentum, scattering angle and vertex) of the combined tracks. The flow chart in Fig. 4.8 illustrates the procedure. Front and back partial tracks are used as input. Unreasonable bridging candidates (partial tracks) are removed by a filter. Front and back partial tracks are then matched with a momentum look-up table. If they cannot be matched, a shooting method, using an RK4 (the 4th-order Runge-Kutta) integration method to propagate ("swim") simulated electrons through the magnetic field is used. 
These procedures are described further in the following sections.

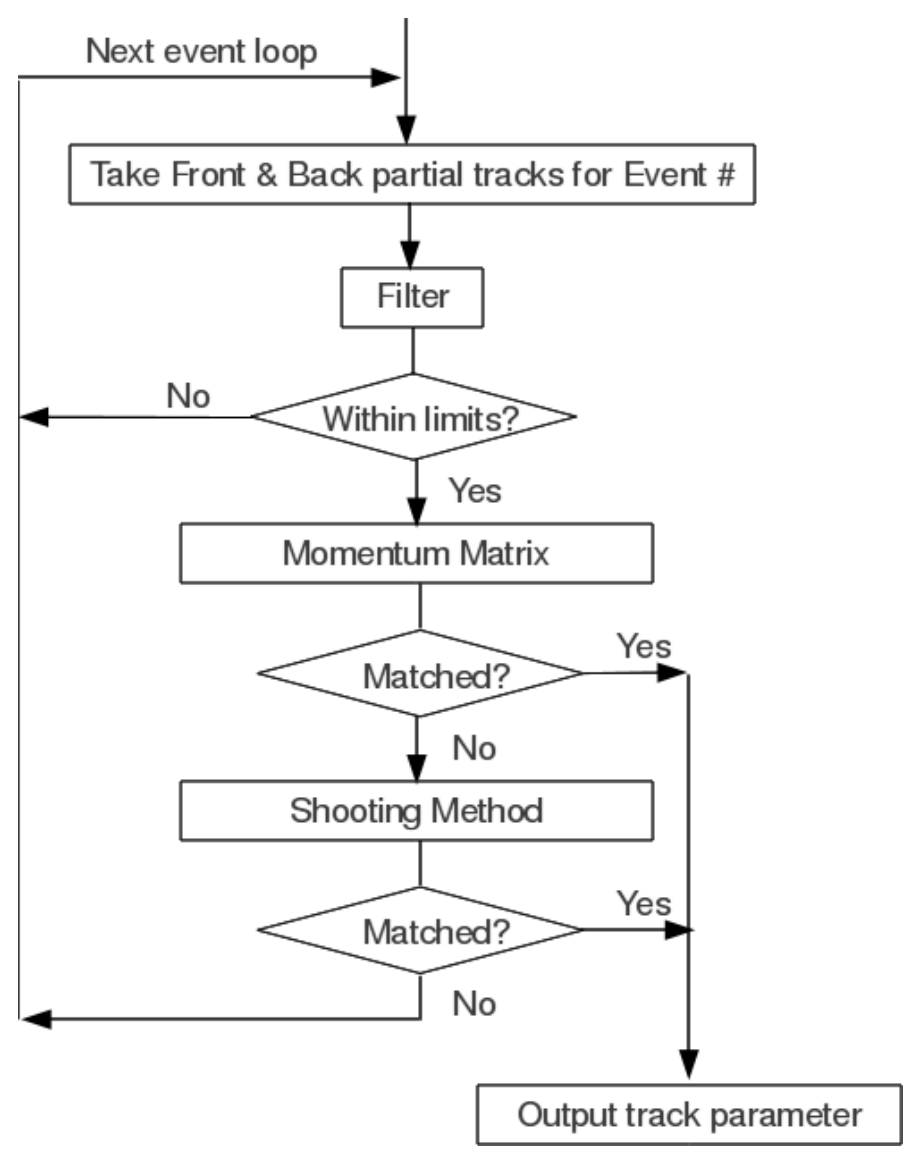

Figure 4.8: Flow chart of partial track bridging.

\subsubsection{Track Filter}

The filter applies the following cuts with the following default values:

- Vertex cut: The vertex of a primary electron defines interaction point of elastic scattering process in the target. Since the incident electrons are not tracked, in the tracking software, it is defined as the point at which the backward extension from a Region 2 partial track passes closest to the beam axis. The $35 \mathrm{~cm}$ long Q-weak liquid hydrogen target is centered at global coordinates of $(0,0,-650 \mathrm{~cm})$. If the z-location of a vertex lies outside the range $[-675 \mathrm{~cm}$, 
$-625 \mathrm{~cm}$ ], QTR considers this partial track not originating from the target, and the track is rejected.

- Scattering angle cut: The elastic e-p scattering angles are selected by the collimators, and are centered at about $8^{\circ}$ with a limit range of about $\left[5^{\circ}, 11^{\circ}\right]$. QTR evaluates the scattering angles by using the Region 2 partial tracks. A scattering angle beyond the range of $\left[3^{\circ}, 13^{\circ}\right]$ is rejected.

- Bending angle cut: The magnetic field of the QTOR spectrometer is dominantly azimuthal $\left(B_{\phi}\right)$, with small components in the radial and axial directions $\left(B_{r}\right.$ and $\left.B_{Z}\right)$; hence, the scattered electrons are mainly deflected in the $\theta$ direction with only small deflection in $\phi$. The $\theta$ and $\phi$ differences of an electron trajectory between the entrance and exit of the spectrometer should be within reasonable limits. In QTR, the limits for $\Delta \theta$ and $\Delta \phi$ are set to $\left[10^{\circ}, 33^{\circ}\right]$ and $\left[-5^{\circ}, 5^{\circ}\right]$, respectively.

- Energy cut: Neglecting any energy loss along the trajectory, the maximum energy of a scattered electron is the beam energy $(1.165 \mathrm{GeV})$. The QTOR spectrometer has a $0.67 \mathrm{~T} \cdot \mathrm{m}$ field integral along the central scattered electron trajectory, which deflects low energy electrons out of the detector acceptance. Simulations indicate that the scattered electron energies accepted by the spectrometer and main detectors are well defined in a range of $[0.90,1.165] \mathrm{GeV}$. If an electron's reconstructed energy lies outside this range, the track is rejected.

\subsubsection{Tracking in the Magnetic Field}

Electron trajectories through the QTOR magnet are deduced by solving the equations of motion for electrons traveling in the magnetic field:

$$
\gamma m_{e} \frac{d^{2} \vec{r}}{d t^{2}}=-e \vec{v} \times \vec{B},
$$


where $\vec{r}$ is the position of the electron, $\vec{v}$ is the velocity, $\vec{B}$ is the magnetic field, and $m_{e}$ is the electron's rest mass. The factor $\gamma=\left(1-v^{2} / c^{2}\right)^{-1 / 2}$ ( $c$ is the speed of light) arises due to relativity. Because $\vec{v}=\frac{d \vec{r}}{d t}$, the $2^{\text {nd }}$ order vector differential equation can be written as a set of coupled $1^{\text {st }}$ order differential equations as:

$$
\begin{array}{lll}
\frac{d x}{d t}=v_{x}, & \frac{d v_{x}}{d t}=-\frac{e}{\gamma m_{e}}\left(v_{y} B_{z}-v_{z} B_{y}\right), \\
\frac{d y}{d t}=v_{y}, & \frac{d v_{y}}{d t}=-\frac{e}{\gamma m_{e}}\left(v_{z} B_{x}-v_{x} B_{z}\right), \\
\frac{d z}{d t}=v_{z}, & \frac{d v_{z}}{d t}=-\frac{e}{\gamma m_{e}}\left(v_{x} B_{y}-v_{y} B_{x}\right),
\end{array}
$$

which are amenable to solution via the $4^{\text {th }}$ order Runge-Kutta integration (RK4) [116]. The initial conditions $\left(x_{0}, y_{0}, z_{0}\right)$ and $\left(v_{0 x}, v_{0 y}, v_{0 z}\right)$ are given by the Region 2 partial track parameters, i.e., the initial coordinates $\left(x_{0}, y_{0}, z_{0}\right)$ are obtained by projecting the Region 2 partial track to a $z_{0}$-plane upstream of QTOR, and the initial velocity is estimated using the electron's momentum $\left(\vec{p}_{0}=\gamma m_{e} \vec{v}_{0}\right)$ and partial track direction, assuming elastic scattering kinematics (see Eqn. 1.21 for reference).

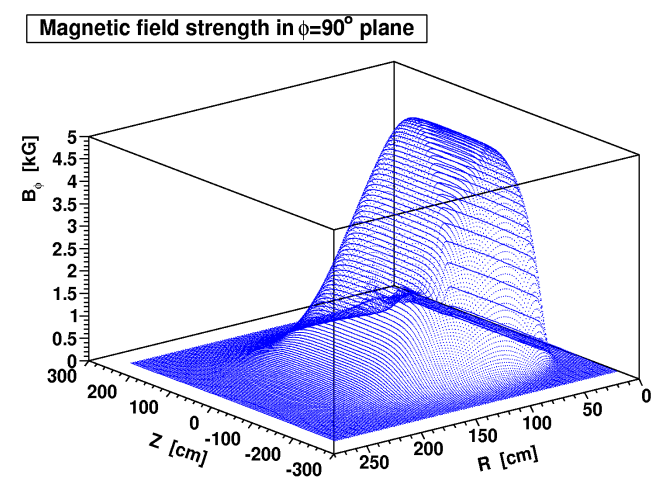

(a)

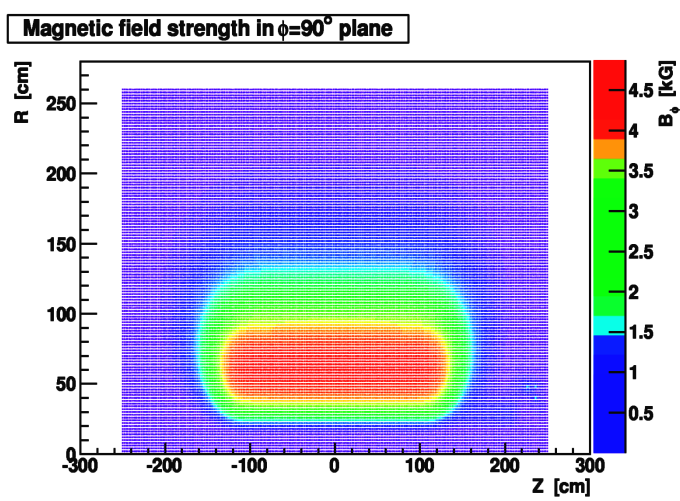

(b)

Figure 4.9: 3D plot (a) and contour plot (b) of the azimuthal magnetic field components $B_{\phi}$ on the median plane between two coils. The QTOR magnetic field is dominated by $B_{\phi}$. Its radial and axial components $B_{R}$ and $B_{Z}$ are small. 
The shape of the magnetic field is depicted in Fig. 4.9. The values are read from a field map that was calculated by numerically integrating the current density distribution in the coils, based on the as-built coil geometry, and verified with a 3D magnetic field mapper [82]. Since the QTOR spectrometer is driven by a steady DC current, the computed field values at a specific coil current can be scaled to other currents.

The resulting field map consists of three magnetic field components $B_{x}, B_{y}$ and $B_{z}$ on lattice points. The lattice points are defined in cylindrical coordinates with separation $\Delta R(2 \mathrm{~cm}), \Delta Z$ $(2 \mathrm{~cm})$ and $\Delta \phi\left(1^{\circ}\right)$. The field values at an arbitrary position between lattice points are obtained from three dimensional trilinear interpolations. Two other interpolation methods (double bilinear and nearest neighbor) are also available as options, permitting a trade-off between computing time and precision.

\subsubsection{The Momentum Matrix}

The momentum of an electron can be determined iteratively in the propagation process as a front partial track is matched to a back partial track. However, propagation through an inhomogeneous magnetic field is time consuming; a look-up-table technique can be used to speed up the bridging process.

To generate this table, a large number of trajectories with well-defined kinematics are calculated, and stored in a look-up table before the analysis. In the table, the recorded back track parameters $\left(R_{b}, \phi_{b}, \theta_{b}, \phi_{b}^{\prime}\right)$ are indexed by the front track parameters $\left(P, R_{f}, \phi_{f}, Z_{v}\right)$ in order to facilitate a rapid search. As the schematic diagram shown in Fig. 4.10 indicates, these parameters are:

- $P$ : the electron momentum (capital ' $P$ ' is used as the magnitude of three momentum in this chapter),

- $R_{f}, \phi_{f}$ : the cylindrical coordinates of the crossing point, when projecting the Region 2 partial tracks upstream to the $z=Z_{f}$ plane,

- $Z_{v}$ : the $z$-location of the scattering vertex, $Z_{v}=Z_{f}-R_{f} / \tan \theta_{f}$, where the $\theta_{f}$ is the scattering 
angle derived from the Region 2 partial track,

- $R_{b}, \phi_{b}$ : the cylindrical coordinates of the crossing point, when projecting the Region 3 partial tracks to the downstream $z=Z_{b}$ plane,

- $\theta_{b}$ and $\phi_{b}^{\prime}$ : the direction angles of the Region 3 partial track, where $\left(\sin \theta_{b} \cos \phi_{b}^{\prime}, \sin \theta_{b} \sin \phi_{b}^{\prime}, \cos \theta_{b}\right)$ defines the direction unit vector.

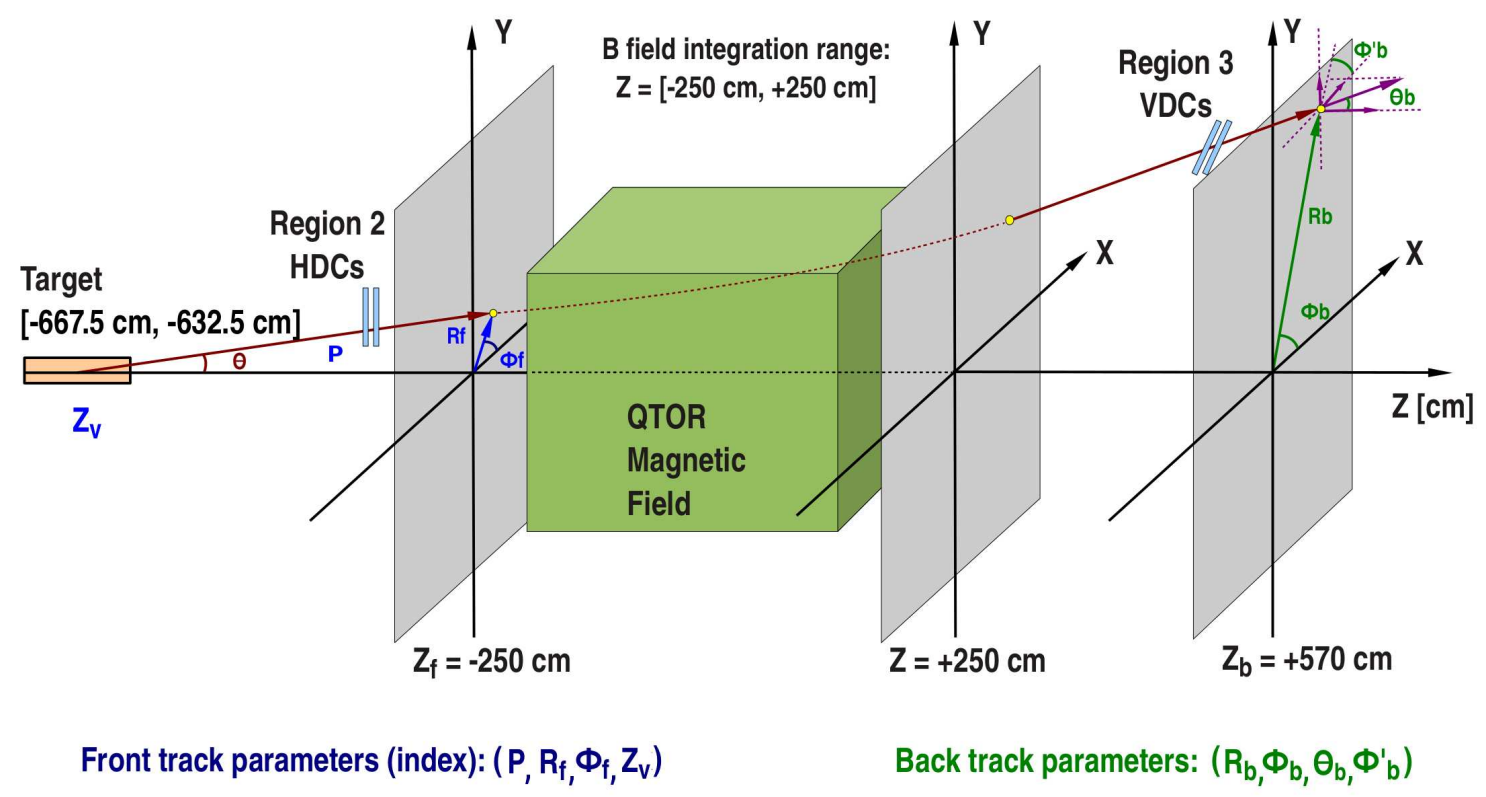

Figure 4.10: Schematic diagram showing the QTR momentum lookup table parameters.

The look-up table is generated by varying the front track parameters on a lattice. Then the electrons are tracked from the $Z_{f}$ plane to the $Z_{b}$ plane. The RK4 algorithm is used to solve the equations of motion. The calculation results in a "momentum matrix" (a 4-dimensional array), with element $M_{P}$ defined as a vector of 4 components, i.e:

$$
M_{P}=\left(R_{b}, \phi_{b}, \theta_{b}, \phi_{b}^{\prime}\right)_{P, R_{f}, \phi_{f}, Z_{v}}
$$

where parameters $P, R_{f}, \phi_{f}$ and $Z_{v}$ are integers, used as indices of the momentum matrix element. 
In order to enable a rapid search to be made using the look-up table, the 4-dimensional array is converted into a 1-dimensional array, i.e. $\left(R_{b}, \phi_{b}, \theta_{b}, \phi_{b}^{\prime}\right)_{P, R_{f}, \phi_{f}, Z_{v}} \rightarrow\left(R_{b}, \phi_{b}, \theta_{b}, \phi_{b}^{\prime}\right)_{i}$, by re-indexing. For example, the following pseudo-code describes a re-indexing algorithm:

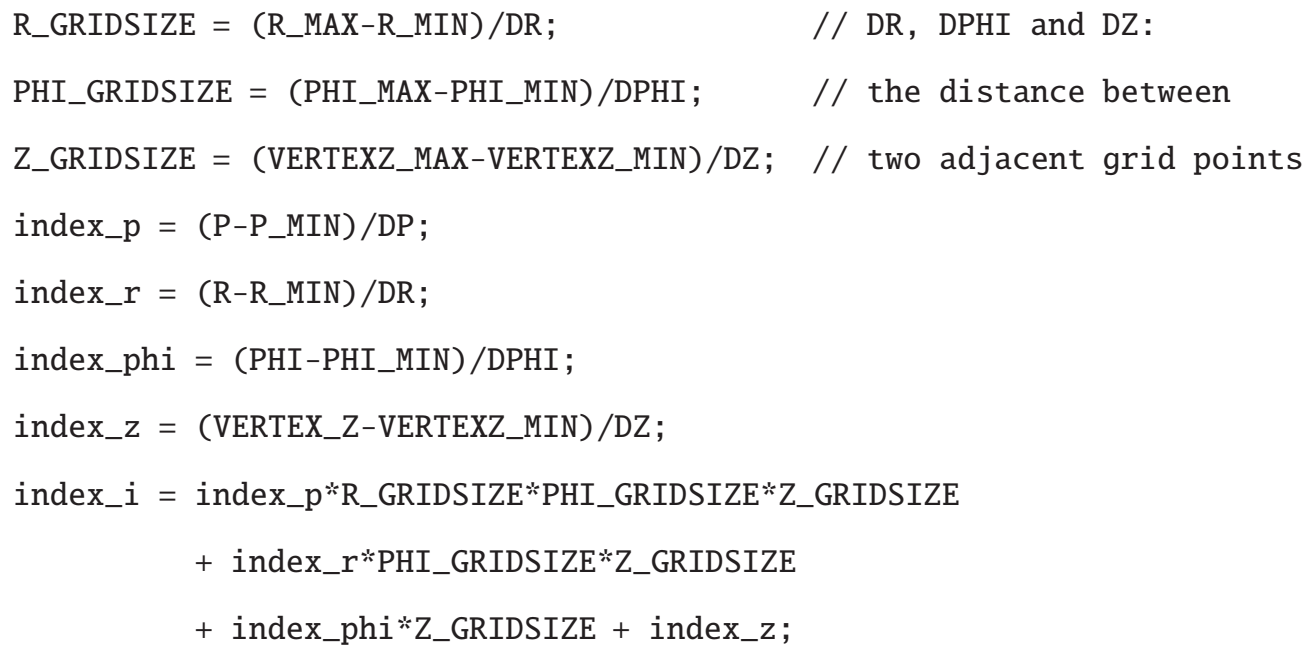

In order to determine the index $i$ of the 1-dimensional array uniquely, the indices $P, R_{f}, \phi_{f}$ and $Z_{v}$ must follow the same sequence given in Eqn. 4.15. The electron's momentum is obtained by searching the table, and performing an interpolation:

- Three parameters $R_{f}, \phi_{f}, Z_{v}$ are derived from the Region 2 partial track. Their closest neighboring integers are used to compute the corresponding index.

- The table is searched with index element $[P]$ varying from $900 \mathrm{MeV} / \mathrm{c}$ to $1160 \mathrm{MeV} / \mathrm{c}$ in $10 \mathrm{MeV} / \mathrm{c}$ steps. This results in a list of matrix elements with different momentum.

- By matching the actual Region 3 partial track parameters to the list, and performing a polynomial interpolation, the track momentum is determined.

This scheme greatly reduces the size of the matrix. By optimizing the step width, the momentum can be determined to an accuracy of $0.1 \%$. 


\subsubsection{Shooting Method}

For tracks which are not within the kinematic region of the momentum matrix, their momenta may be determined by a shooting method [116]. The adjustable parameter in the shooting method is the electron's momentum $P$. Taking into account the electron energy loss in the target, the initial value of $P$ is estimated using the e-p scattering kinematics. By varying $P$, and computing the trajectory with the RK4 algorithm, the front and back partial tracks are matched at the $Z_{b}$ plane. The NewtonRaphson method [116] is used so that quick convergence is obtained.

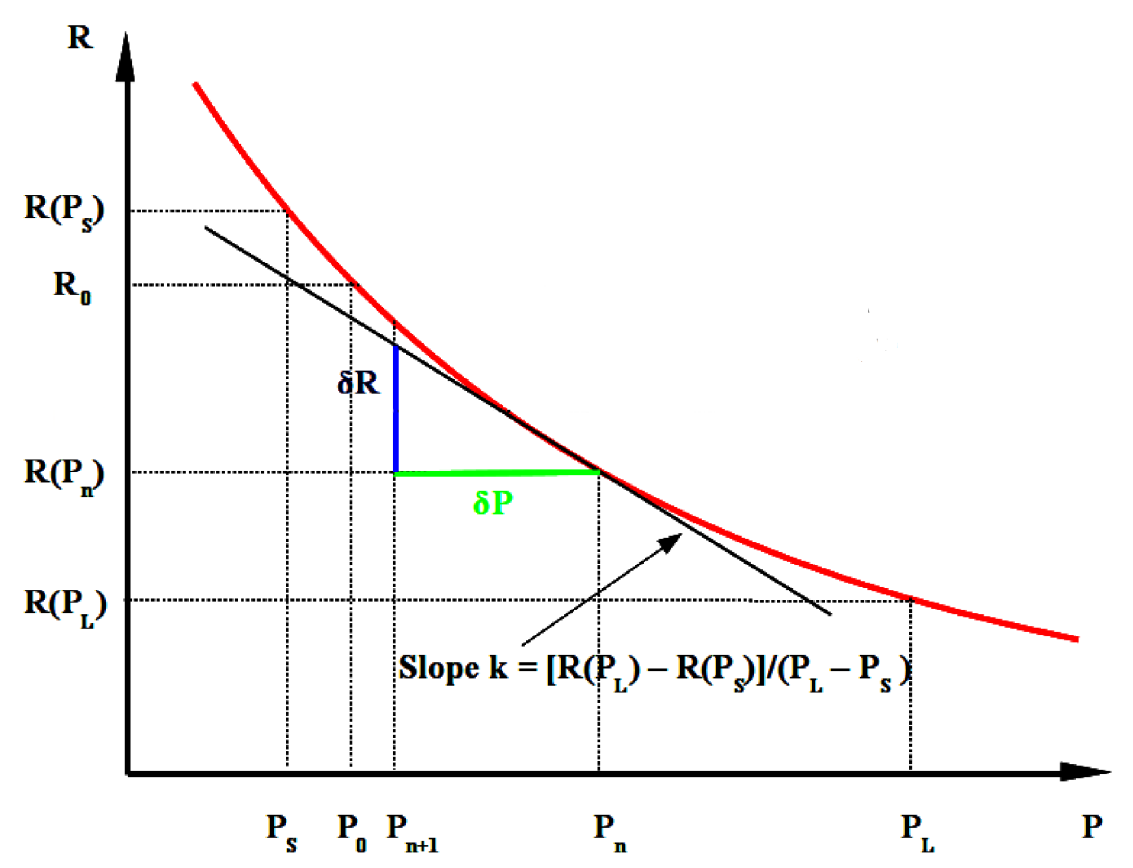

Figure 4.11: Shooting method using Newton iteration.

The shooting method with Newton iteration is illustrated in Fig. 4.11. A VDC partial track intersects a downstream plane at a radial distance $R_{0}$ to the beam center. For an electron with momentum $P_{n}(n=1,2,3 \ldots)$, its trajectory will intersect the same downstream plane at a radial distance $R\left(P_{n}\right) . R$ is a monotonically decreasing function of $P$, and the slope at $P_{n}$ is

$$
k=\frac{d R}{d P} \simeq \frac{R\left(P_{L}\right)-R\left(P_{S}\right)}{P_{L}-P_{S}}=\frac{R\left(P_{n}+\Delta P\right)-R\left(P_{n}-\Delta P\right)}{2 \Delta P} .
$$


The correction for $P_{n}$ can be estimated by

$$
\delta P=\delta R / k
$$

where $\delta R=\frac{1}{2}\left[R\left(P_{L}\right)+R\left(P_{S}\right)\right]-R_{0}$ and $\delta P=P_{n}-P_{n+1}$, leading to the expression for the iteration:

$$
\begin{aligned}
& \frac{1}{2}\left[R\left(P_{L}\right)+R\left(P_{S}\right)\right]-R_{0}=\left(P_{n}-P_{n+1}\right) \frac{R\left(P_{L}\right)-R\left(P_{S}\right)}{2 \Delta P} \\
& P_{n+1}=P_{n}-\frac{\Delta P\left[R\left(P_{n}+\Delta P\right)+R\left(P_{n}-\Delta P\right)-2 R_{0}\right]}{R\left(P_{n}+\Delta P\right)-R\left(P_{n}-\Delta P\right)} .
\end{aligned}
$$

Defining a resolution variable res, the iterations end when a resolution condition $\left|R\left(P_{n}\right)-R_{0}\right|<$ res (in units of $\mathrm{cm}$ ) is satisfied at the $Z_{b}$ plane. The resolution res was set to $0.5 \mathrm{~cm}$ during testing, and was adjusted to meet different accuracy requirements.

\subsubsection{Momentum Transfer Determination}

When determining the momentum transfer, the energy loss of the electron in the target and target windows must be taken into account. In the target, the pre- and post-scattering energy losses $\left(\delta E_{\text {pre }}\right.$ and $\delta E_{\text {post }}$ ) can be calculated based on Monte Carlo simulations (see Fig. 4.12) and the electron's path lengths before and after scattering, which requires the determination of the scattering vertex. In QTR, the scattering vertex is found by extension of the Region 2 partial track back to the target region.

The 4-momentum transfer squared is computed by:

$$
Q^{2}=4 E_{0} E^{\prime} \sin ^{2} \frac{\theta}{2}=4\left(E_{b}-\delta E_{\mathrm{pre}}\right)\left(E+\delta E_{\mathrm{post}}\right) \sin ^{2} \frac{\theta}{2}
$$

where the post-scattering energy $E^{\prime}$ at vertex is calculated from the reconstructed momentum $P$ $(P \simeq E)$ and the post-scattering energy loss $\delta E_{\text {post }}$ (the average value $\left\langle\delta E_{\text {post }}\right\rangle \sim 24 \mathrm{MeV}$ ), i.e. $E^{\prime}=E+\delta E_{\text {post }}$; the pre-scattering energy $E_{0}$ at vertex is calculated from the measured beam energy $E_{b}$ and pre-scattering energy loss $\delta E_{\text {pre }}$ (the average value $\left\langle\delta E_{\text {pre }}\right\rangle \sim 14 \mathrm{MeV}$ ), i.e. $E_{0}=E_{b}-\delta E_{\text {pre }}$ 
(see Fig. 4.13 for a simulated pre-scattering energy distribution); $\theta$ is the reconstructed scattering angle. These variables are indicated in Fig. 4.12.

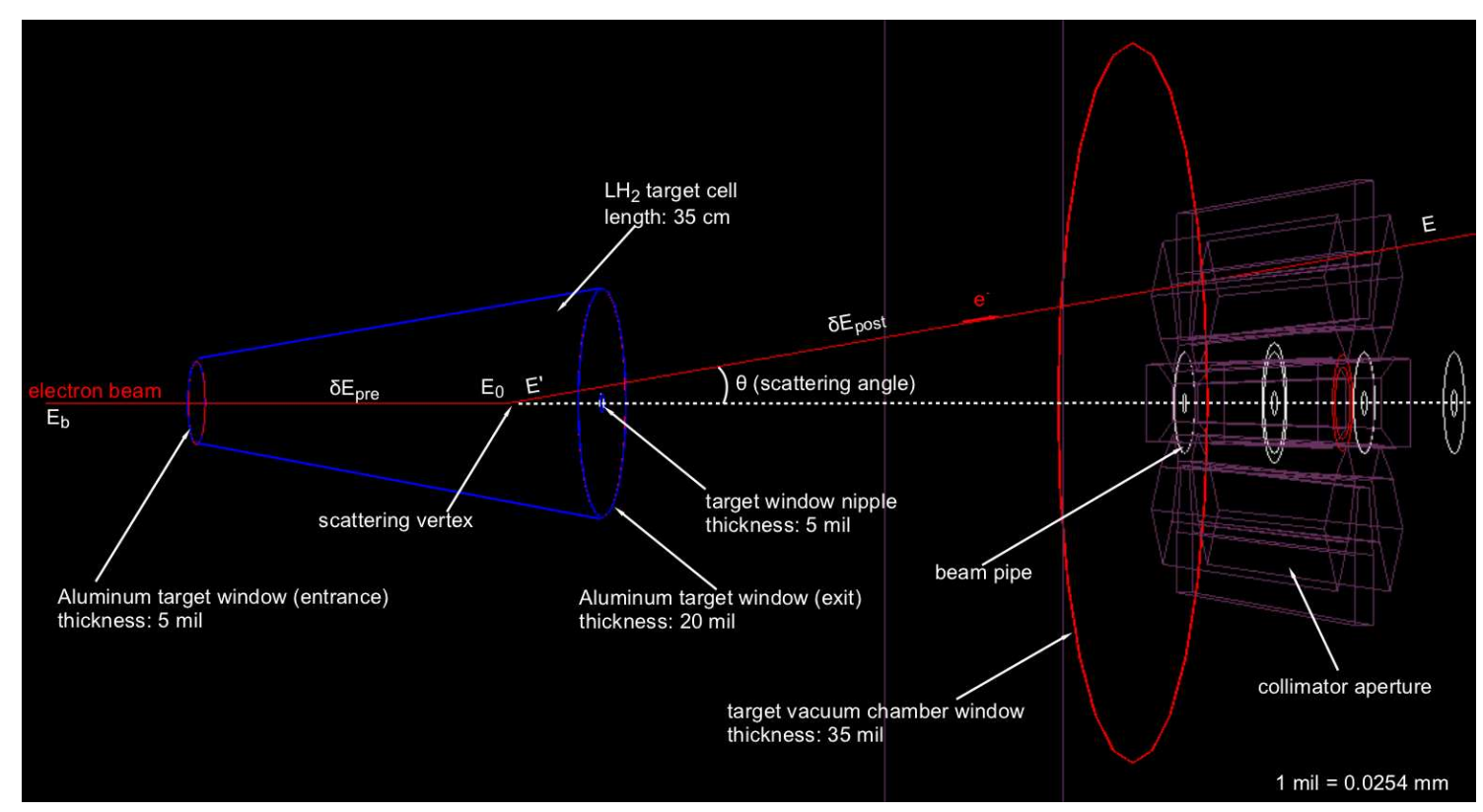

Figure 4.12: Outline of the energy loss simulation using the Q-weak Geant4 Monte Carlo simulation program (QweakSimG4).

The $Q^{2}$ could be determined by $E_{0}, E^{\prime}$ and $\theta$, according to Eqn. 4.20. However, from Eqn. 1.21, only two of them are independent variables. Therefore, any two of the three variables may be used for $Q^{2}$ determination in QTR. During earlier QTR tests, $Q^{2}$ was determined based on $E^{\prime}, \theta$ and $\left\langle\delta E_{\text {post }}\right\rangle$. Recently, the tracking analyzer was changed to use $E_{0}, \theta$ and $\left\langle\delta E_{\text {pre }}\right\rangle$ for $Q^{2}$ determination. The precision of the determined $Q^{2}$ then mainly relies upon the precision of the $E_{b}$ measurement, Region 2 partial track determination and the estimation of $\delta E_{\text {pre }}$ in Monte Carlo simulations.

The $Q^{2}$ must be calculated from valid tracks which generate a Čerenkov light signal in the main Čerenkov detectors. The light-weighted $\left\langle Q^{2}\right\rangle$ is determined by

$$
\left\langle Q^{2}\right\rangle=\frac{\sum_{i}(\# \mathrm{PE})_{i} Q_{i}^{2}}{\sum_{i}(\# \mathrm{PE})_{i}},
$$




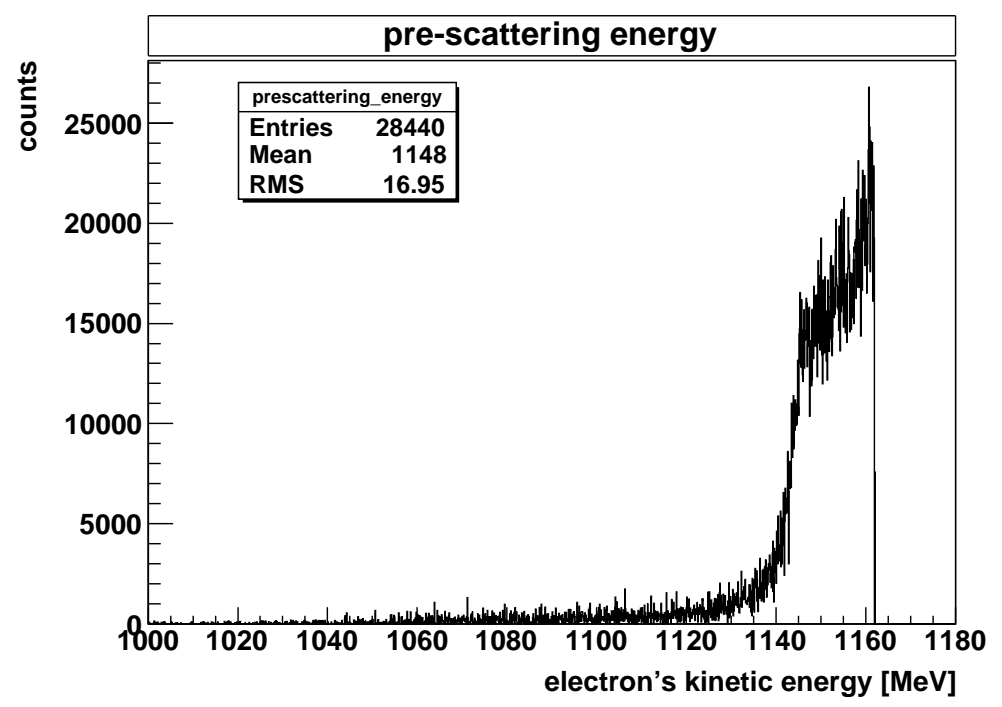

Figure 4.13: A simulated pre-scattering energy $\left(E_{0}\right)$ distribution at vertexes in the $\mathrm{LH}_{2}$ target. The average pre-scattering energy loss is $\left\langle\delta E_{\text {pre }}\right\rangle=E_{b}-\left\langle E_{0}\right\rangle=1162-1148 \mathrm{MeV}=14 \mathrm{MeV}$.

where $Q_{i}^{2}$ is the 4-momentum transfer squared for track $i$, and \#PE is the yield of photo-electrons in the main detector associated with track $i$. \#PE can be determined from the main detector's single photo-electron calibration and ADC pulse height.

\subsection{Bridging Performance Test}

The Q-weak Geant4 Monte Carlo simulation program, known as QweakSimG4, was used to provide simulated events for QTR, to test its performance. Fig. 4.14 shows some simulated tracks. Monte Carlo simulated data are particularly well-suited for QTR tests, because this method generates known kinematic information for each event. In addition, the QweakSimG4 can simulate the

light response of the main detector, so that the light-weighted $\left\langle Q^{2}\right\rangle$ can be evaluated and compared with experimental results. 


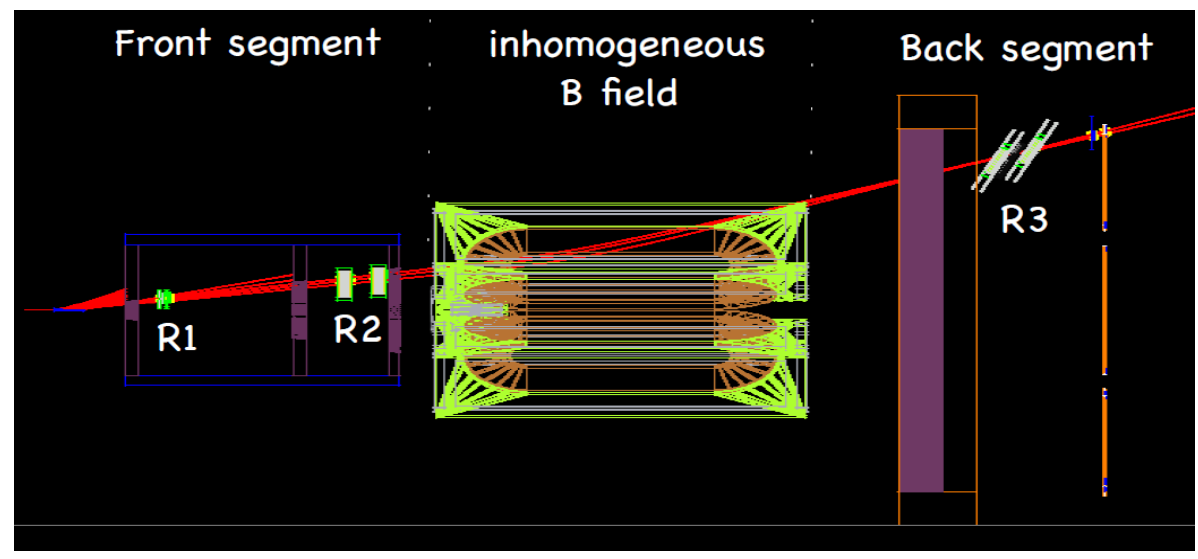

Figure 4.14: The simulated tracks and three regions of tracking detectors in QweakSimG4.

\section{Bridging Speed}

One of the considerations in the QTR development is the computing speed of the software. In QTR, the bridging process is one of the most time consuming parts. The computing times for two bridging algorithms are compared in Fig. 4.15. Using a $2 \mathrm{GHz}$ single CPU, it takes about $0.06 \mathrm{~ms}$ to determine the momentum of a track by searching the look-up table, and $6.5 \mathrm{~ms}$ by using the shooting method with an integration step size of $1 \mathrm{~cm}$ [117].

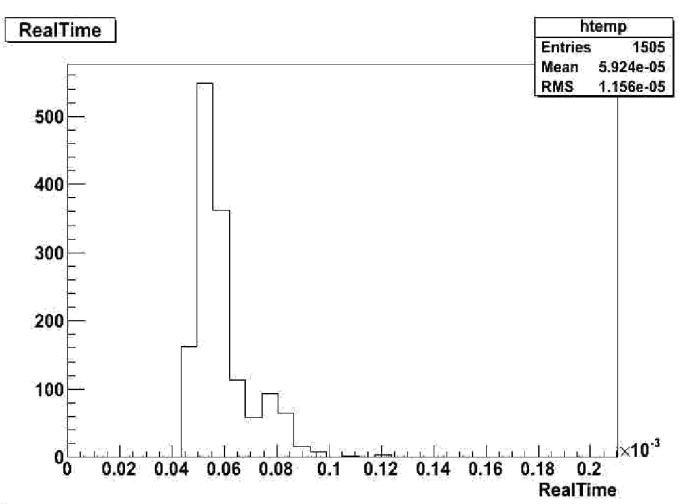

(a)

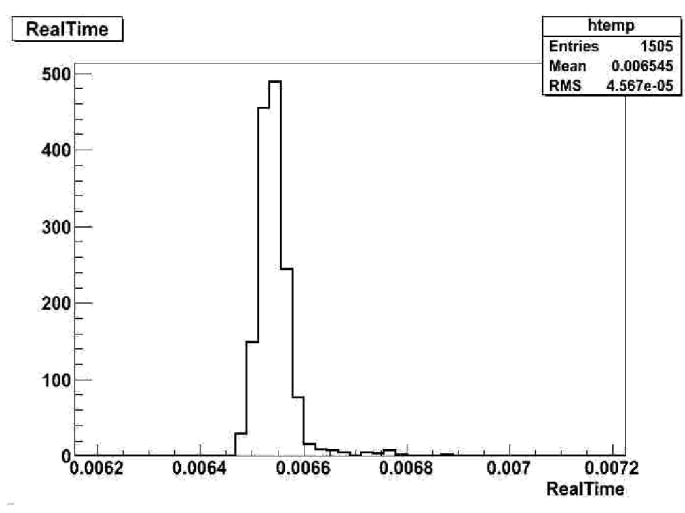

(b)

Figure 4.15: Computing time comparison for different bridging algorithms. (a) Computing time (seconds) for the look-up table method. (b) Computing time (seconds) for the shooting method. 


\section{Residuals}

The momentum reconstruction was tested with "ideal" simulated events for which multiple scattering was switched off in the simulation. In this way each simulated event provided a clean partial track pair for QTR. In order to mimic the effects of detector resolution and partial track finding accuracy, the ideal partial tracks in Region 2 and Region 3 chambers were smeared according to Gaussian distributions in positions and angles with $\sigma_{\text {position }}=500 \mu \mathrm{m}$ and $\sigma_{\text {slope }}=0.1$ degree. The test results with and without this smearing are compared in Fig. 4.16. Referring to this figure, the momentum residual represents the momentum difference between reconstructed events and generated events. It was found that the mean of momentum residuals could be determined to $<0.1 \%$, with a RMS width of $\sim 0.5 \%$ of the electron's momentum even with smeared tracks.

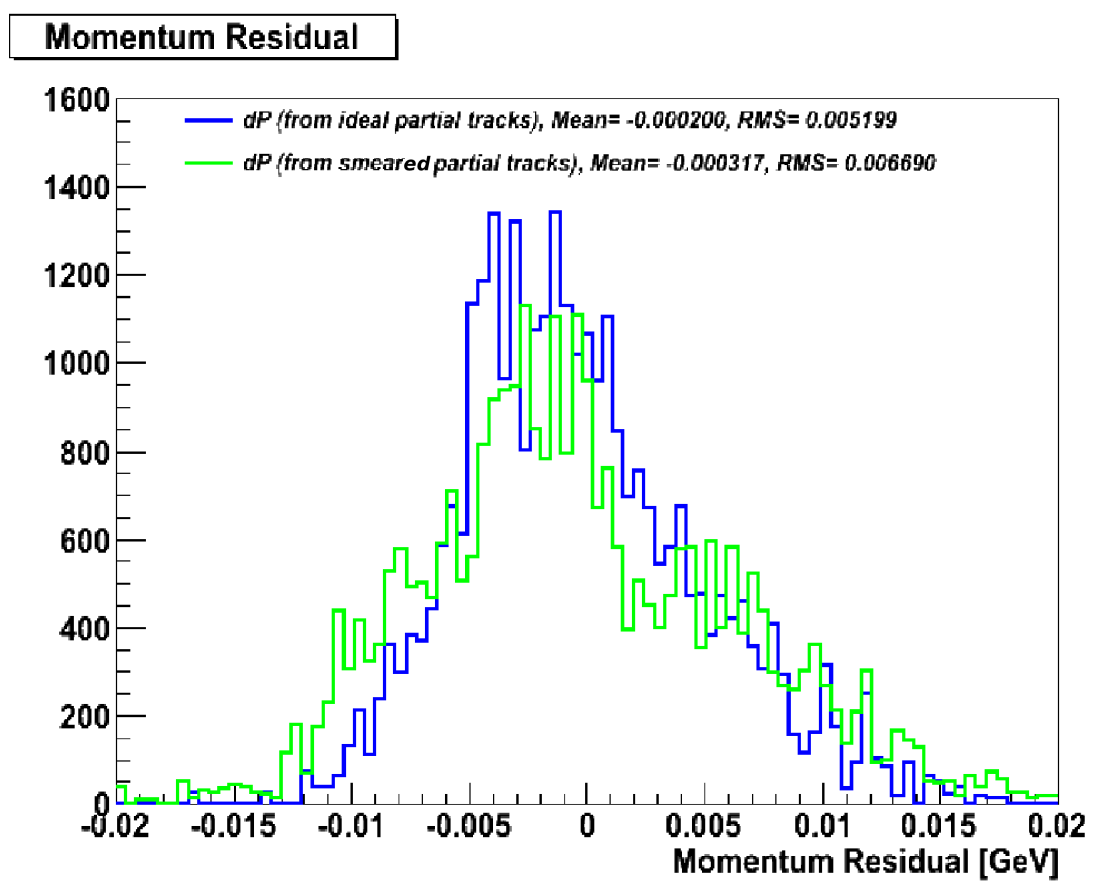

Figure 4.16: Distribution of momentum residuals for "ideal" simulation events with and without resolution smearing for Region 2 and Region 3 partial tracks. The offset of the mean momentum residuals is due to the systematic bias introduced by simulation.

The momentum reconstruction was further tested with "realistic" Monte Carlo events, with the 
multiple scattering process turned on and resolution smearing on. The generated and reconstructed momentum distributions are compared in Fig. 4.17. In this more realistic case, the mean of momentum residuals could also be determined to $<0.1 \%$, with a RMS width of $\sim 1 \%$ of the electron's momentum (see Fig. 4.18).

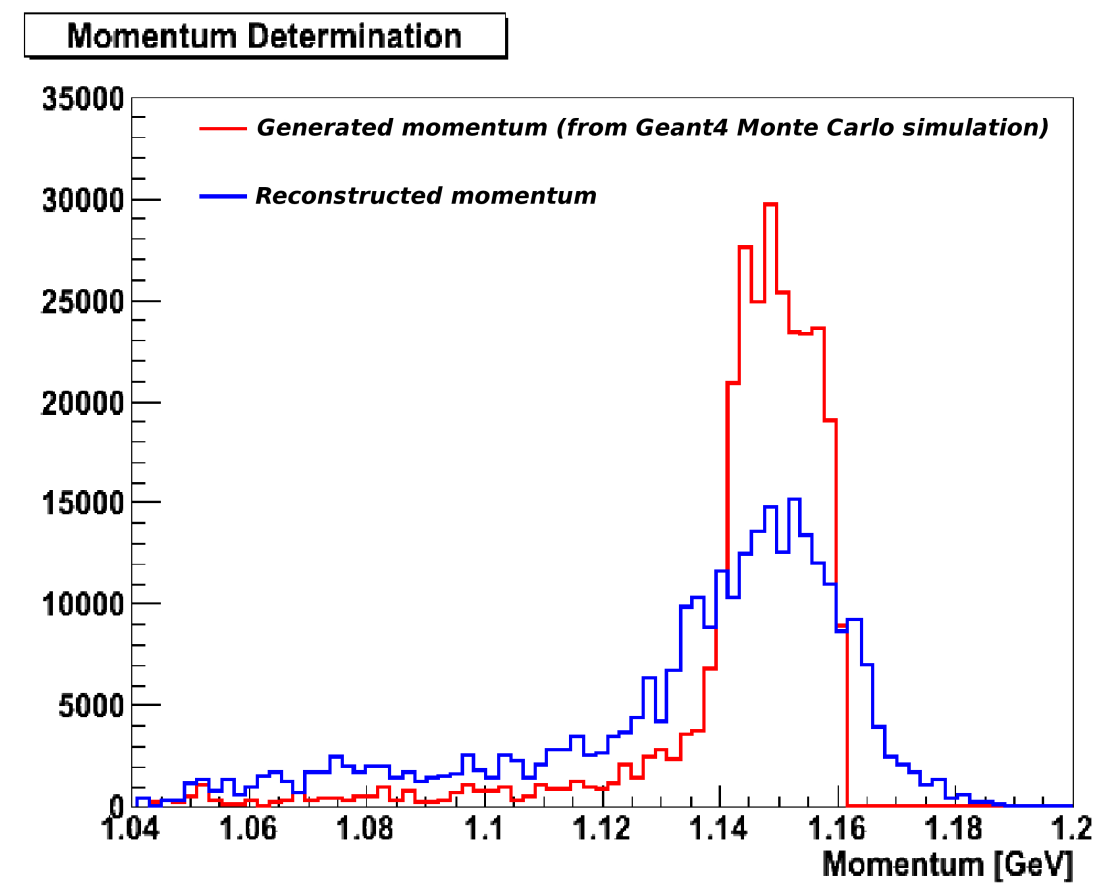

Figure 4.17: Comparison of simulated momentum distribution (red) and reconstructed momentum distribution (blue).

The quality of the algorithm was checked by examining reconstructed track parameters. The mean residuals of four major track parameters, extrapolated to the focal plane, are shown in Table 4.1 .

The tests with simulated events demonstrate good performance of the bridging algorithm. The results showed furthermore that high precision can be reached by either the look-up table or the shooting method. 


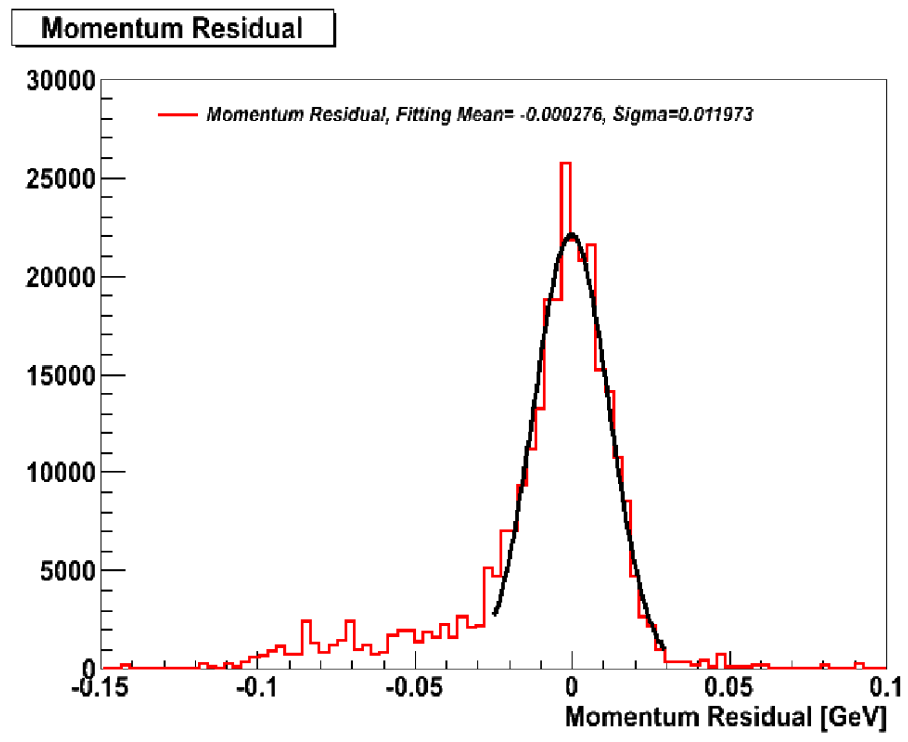

Figure 4.18: Distribution of momentum residuals for "realistic" simulation events.

\begin{tabular}{cccc}
\hline \hline Parameter & Residual & Ideal Event & Ideal Event with Smeared Tracks \\
\hline $\begin{array}{cccc}\text { Directional Angle } \\
(\theta, \phi)\end{array}$ & $\Delta \theta$ [degree] & $0.063 \pm 0.016$ & $0.15 \pm 0.10$ \\
& $\Delta \phi$ [degree] & $0.00 \pm 0.16$ & $-0.71 \pm 0.93$ \\
\hline Hit Position & $\Delta R[\mathrm{~cm}]$ & $0.44 \pm 0.12$ & $0.27 \pm 0.56$ \\
$(R, \Phi)$ & $\Delta \Phi[$ degree] & $0.00 \pm 0.13$ & $-0.61 \pm 0.77$ \\
\hline \hline
\end{tabular}

Table 4.1: Mean residuals of different track parameters for ideal events with or without smeared partial tracks. The uncertainties shown in the table are RMS widths.

\section{Reconstruction Efficiency}

The overall QTR reconstruction efficiency is defined as:

$$
\epsilon=\epsilon_{\text {front }} \cdot \epsilon_{\text {bridge }} \cdot \epsilon_{\text {back }},
$$


where $\epsilon_{\text {front }}$ and $\epsilon_{\text {back }}$ are the front and back partial track finding efficiencies, respectively; $\epsilon_{\text {bridge }}$ is the partial track bridging efficiency. The quantities $\epsilon_{\text {front }}$ and $\epsilon_{\text {back }}$ represent the probability that a front/back partial track is recognized by the partial track finding program, defined as

$$
\epsilon_{\text {front } / \text { back }}=\frac{\text { Number of correctly found partial tracks }}{\text { Number of actual partial tracks }} .
$$

$\epsilon_{\text {bridge }}$ is the probability that front and back partial track pairs are matched by the partial track bridging program, defined as

$$
\epsilon_{\text {bridge }}=\frac{\text { Number of matched partial track pairs }}{\text { Number of actual partial track pairs }}
$$

Testing with Monte Carlo simulated events, the QTR bridging efficiency is $98 \%$ for the realistic events. The partial track finding efficiencies were not carefully tested in simulation.

\subsection{Preliminary Tracking Results}

Measuring the reconstruction efficiency is difficult with real data, because the raw data are strongly contaminated by backgrounds. However, Ref. [115] discussed a scheme to test the efficiency with real data by varying parameters so as to change different qualities of reconstruction. For instance, by varying the number of maximal allowed missing hits, the chamber efficiency can be calculated.

In dedicated runs during the Q-weak Run I period, event mode data were taken for all octants with the Region 2 and Region 3 chambers. The first attempt at reconstructing tracking results from actual data showed very reasonable values for track parameters and reconstructed $Q^{2}$, although with low efficiency [118]. In that analysis, a large number of partial tracks were rejected by the track filter, introduced in Section. 4.4.1. With steady improvements to the partial track finding efficiency and resolution in Region 2 and Region 3, the QTR reconstruction efficiency has increased. Some preliminary results from the most recently updated analyzer are given below as examples. To check the performance of the track finding code, the track filter was not activated during this analysis. All 
the examples below are from the analysis of one octant (octant 5) data in tracking run 8658, which were acquired at a beam current of $50 \mathrm{pA}$.

Since the partial tracks are straight lines in near zero magnetic field regions, they can be directly projected to planes at different $z$ locations, to provide a visual check of the scattered beam profiles. As an example, Fig. 4.19 shows the track projections from upstream and downstream partial tracks. Most events $(\sim 98 \%)$ are within the outlines of the collimator opening and main detector quartz bar.

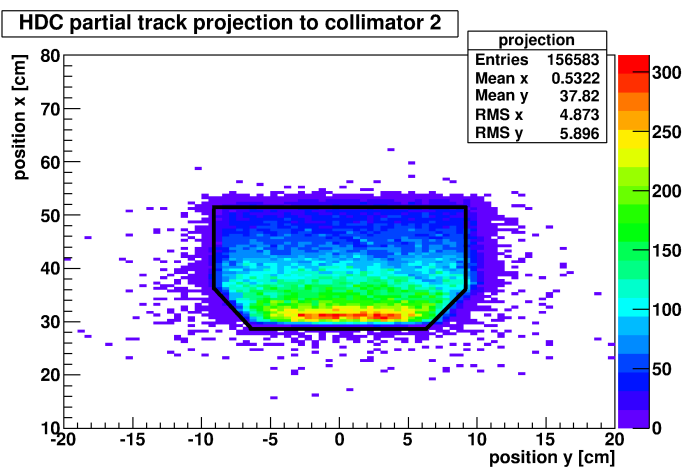

(a) HDC partial track projection to collimator 2

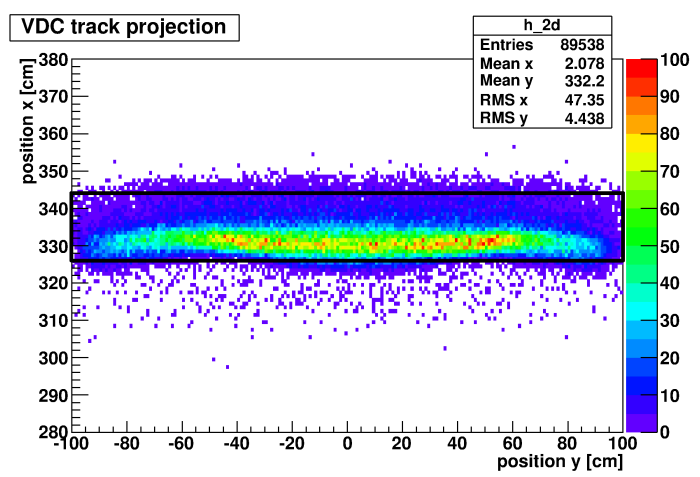

(b) VDC partial track projection to main detector

Figure 4.19: Partial track projections. (a) The upstream partial track projections to the upstream aperture of the primary collimator $(z=-385.7 \mathrm{~cm})$. The black frame outlines the collimator opening. The color scale indicates the number of HDC (Region 2) partial tracks. (b) The downstream partial track projections to the main detector central $z$-plane $(z=577.9 \mathrm{~cm})$. The black frame outlines the main detector quartz bar. The color scale indicates the number of VDC (Region 3) partial tracks.

Fig. 4.20 shows the projected scattering vertex before and after track bridging. The vertex resolution is mainly determined by the HDC partial track parameters. There are $50 \%$ of scattering vertices lying outside of the liquid hydrogen target region (between $z=-632.5 \mathrm{~cm}$ and $z=-667.5 \mathrm{~cm}$ ) from the difference in the number of events between Fig. 4.20 (a) and (b). This implies that the vertex reconstruction needs to be further improved. A scintillation fiber detector for improving vertex determination in Q-weak Run II is being considered, to replace the malfunctioning GEM chambers [119]. The reconstructed scattering angle and the electron's kinetic energy are shown in Fig. 4.21 (a) and Fig. 4.21 (b), respectively. The mean values of the reconstructed 


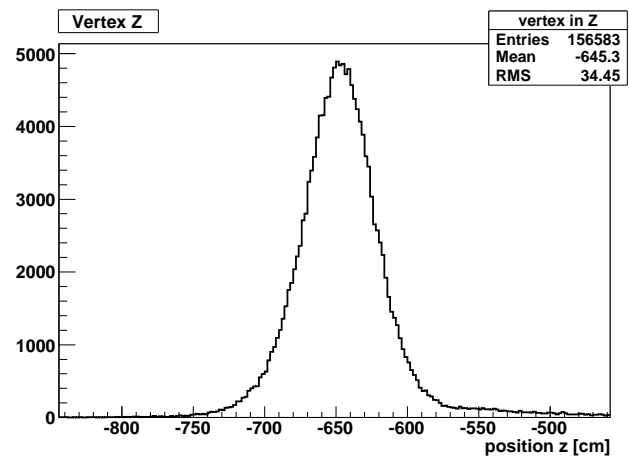

(a)

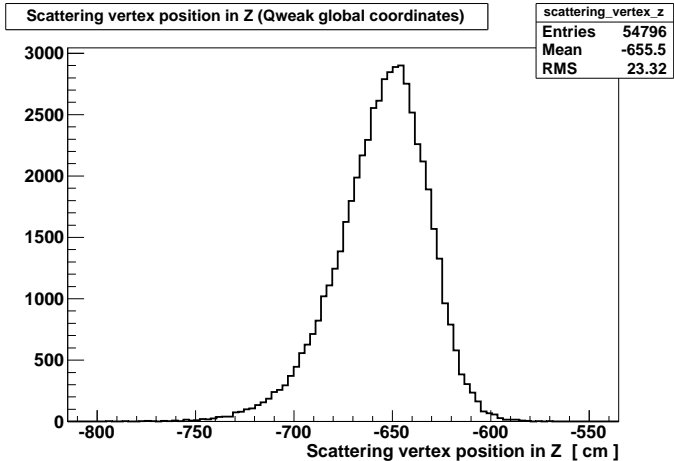

(b)

Figure 4.20: Projected scattering vertices before and after track bridging. (a) Scattering vertices projected by HDC partial tracks before bridging. (b) Scattering vertices projected by the bridged tracks. The distribution is narrowed after bridging, implying that some HDC background tracks are rejected.

scattering angle $\left(7.61 \pm 0.01^{\circ}\right)$ and kinetic energy $(1.113 \pm 0.001 \mathrm{GeV})$ are close to the simulated results $\left(7.782 \pm 0.007^{\circ}\right.$ and $\left.1.111 \pm 0.001 \mathrm{GeV}\right)$. However, the differences between the reconstructed and the simulated distributions need further investigation.

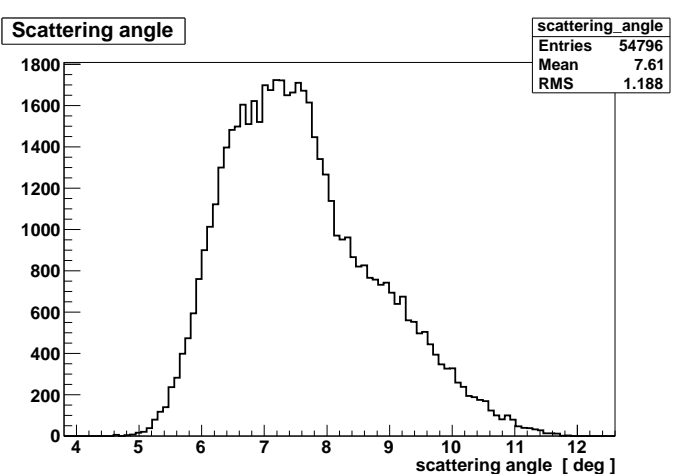

(a)

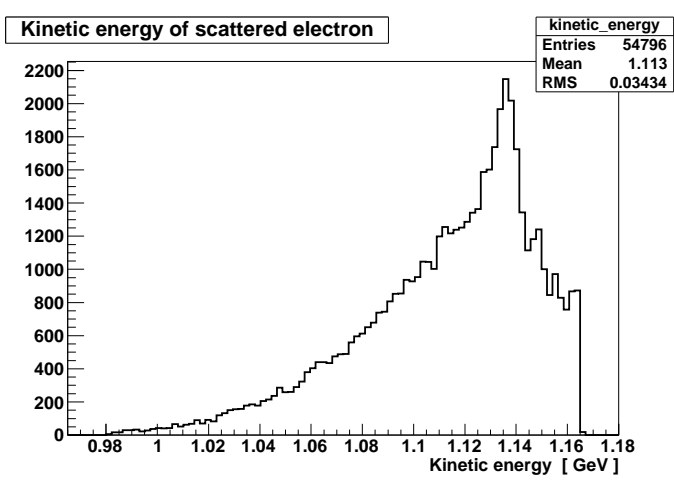

(b)

Figure 4.21: The reconstructed scattering angle (a) and electron kinetic energy (b).

When correlating the reconstructed tracks with the main detector PMT signals, the track dependent light response of the main detector can be mapped out. The hit distribution, light distribution, 


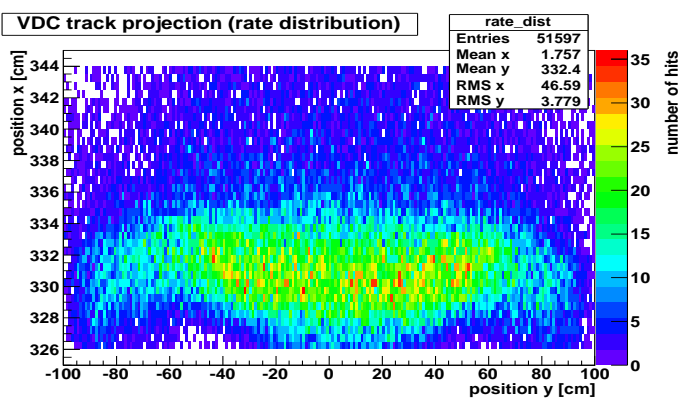

(a) Number of hits distribution

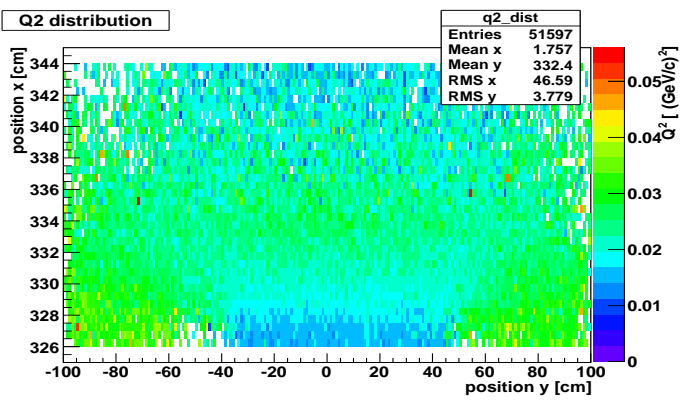

(c) $Q^{2}$ distribution

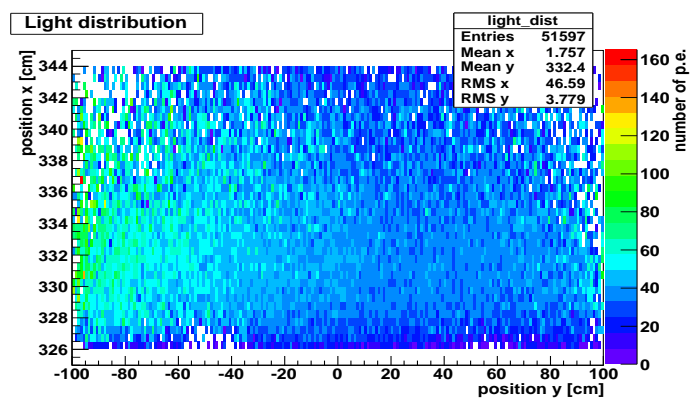

(b) light yield distribution

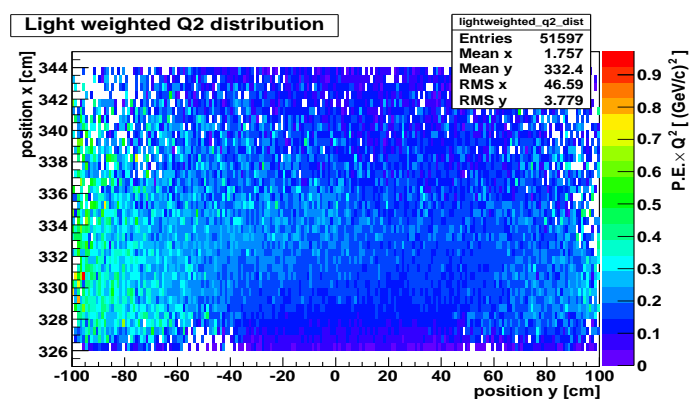

(d) Light-weighted $Q^{2}$ distribution

Figure 4.22: Distributions of number of hits, light yield, $Q^{2}$ and light-weighted $Q^{2}$ on main detector 5 (from tracking run 8658).

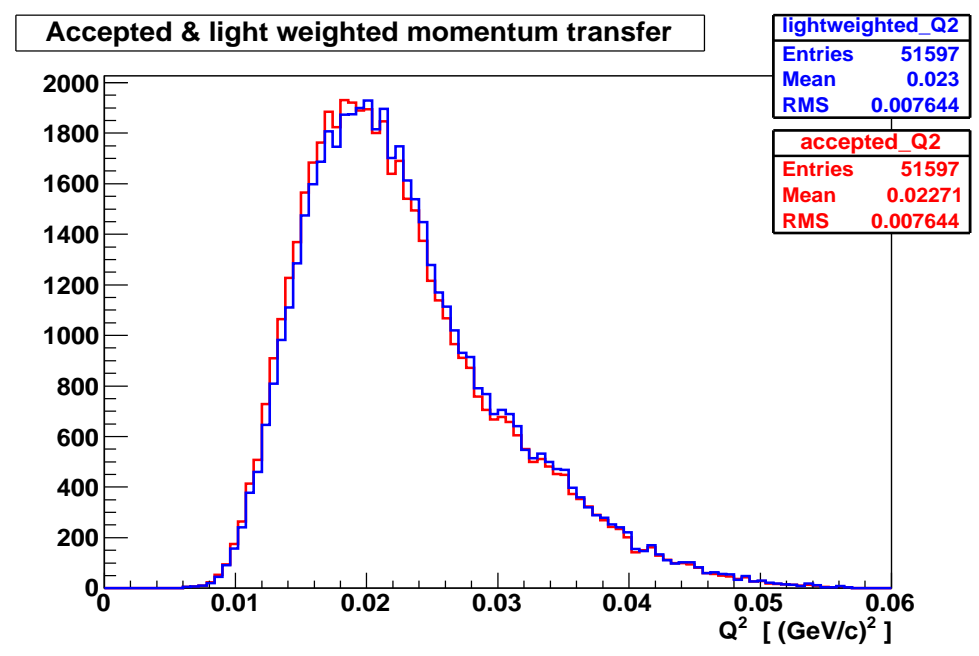

Figure 4.23: Reconstructed squared momentum transfer (red) and light-weighted momentum transfer (blue). The light weighted distribution is shifted to higher $Q^{2}$ by $1.3 \%$ on average. 
$Q^{2}$ distribution and light-weighted $Q^{2}$ distribution of the main detector are exhibited in Fig. 4.22. The number of hits distribution in Fig. 4.22 (a) shows a 'moustache' shape, which is in agreement with simulation. The light yield and $Q^{2}$ distributions Fig. 4.22 (b) and (c) also approximately agree with simulations: more photo-electrons are obtained when the electrons strike the ends of a main detector quartz bar, closer to PMTs; higher $Q^{2}$ value events also lie close to the quartz bar ends. The light-weighted $Q^{2}$ distribution in Fig. 4.22 (d) is a combination of the distributions in Fig. 4.22 (a), (b) and (c). The histograms of reconstructed $Q^{2}$ and light-weighted $Q^{2}$ shown in Fig. 4.23 indicate that the light weighting shifts the value of $\left\langle Q^{2}\right\rangle$ up by about $1.3 \%$. The most recent simulation with pre-radiated main detectors indicates that the light-weighted $\left\langle Q^{2}\right\rangle$ is about $1.4 \%$ larger than the value of $\left\langle Q^{2}\right\rangle$ without light weighting.

Continuous efforts are still being made to further improve the track finding efficiency and precision of partial track reconstruction [120]. What is reported here represents the results available at the time of writing. 


\section{Chapter 5}

\section{Scanner Data Analysis}

In this chapter, scanner data analysis will be discussed, and some preliminary results will be presented (for a complete list of the scanner runs taken during Q-weak Run I, see Table B.1 in Appendix B). The discussions here are mainly focused on the evaluation of the scanner detector performance and studies of $Q^{2}$ extrapolation to high beam currents. Other scanner applications, namely spectrometer optics measurements for $\mathrm{LH}_{2}$ and $\mathrm{Al}$ targets, will also be discussed.

\subsection{Focal Plane Flux Profile}

The scanner detector measures the scattered electron rates near the focal plane of the spectrometer across the fiducial area of the main detector in octant 7 (the bottom octant). Rate distributions may be measured at various beam currents. The rate distributions are obtained based on measured singles rates, accidental coincidence rates (randoms) and total coincidence rates from the two photomultiplier tubes, counted independently by four scaler channels as described in Section 3.11. 


\subsubsection{Raw Rate Distribution}

\section{Coincidence and Accidental Coincidence Rate Maps}

Čerenkov light generated by one electron which passes through both quartz elements is detected by the two scanner PMTs simultaneously. These events provide the true coincidence rate $R_{\text {coin }}$. A false, or accidental coincidence rate $R_{a c c}$ occurs when two unrelated events happen to appear for each detector arm within the selected time window. As explained in Chapter 3, accidental coincidences are estimated by counting another coincidence channel formed by the two PMTs, with one signal being delayed outside the time resolution of the system.

Because the rates measured by the scanner detector are functions of its position $(x, y)$, we use $R(x, y)$ to denote the 2-dimensional rate distribution in the scan plane (called a rate map). The raw coincidence rate map and measured accidental rate map from run 6616 at a beam current of $50 \mu \mathrm{A}$ are shown as examples in Fig. 5.1. The rate maps in the figure are 2D histograms, whose bin contents are denoted by $R_{t o t}\left(x_{i}, y_{j}\right)$ and $R_{a c c}\left(x_{i}, y_{j}\right)$ for the total coincidence and accidental rates, respectively. The $x_{i}$ and $y_{j}$ represent the coordinates ${ }^{1}$ of the quartz elements for the $(i, j)$ bin in the 2D histogram. The scanner's nominal active area for normal incidence, $1 \mathrm{~cm}^{2}$, was selected as the bin size, within which the average rate was recorded. The entire map was made following the vertical scan path as shown in Fig. 3.28. The plots in Fig. 5.1 show that the accidental intensities near the center of the main detector are very high $\left(R_{a c c}\left(x_{i}, y_{j}\right) / R_{t o t}\left(x_{i}, y_{j}\right) \sim 66 \%\right)$. In order to obtain the electrons' true rate distribution, the raw rate map must be corrected for accidental coincidences, and furthermore deadtime effects, which are grouped together and referred to as rate corrections in the following discussion.

Beam Current Normalization and Quality Cuts With a nominal motion speed of $5 \mathrm{~cm} / \mathrm{s}$, the scanner system can complete a full bar scan within half an hour. During this data taking period, beam current changes, beam trips or excursions may occur. The beam intensity dependence of the

${ }^{1}$ Unless explicitly noted, all the coordinates in this chapter use Q-weak global coordinates. 


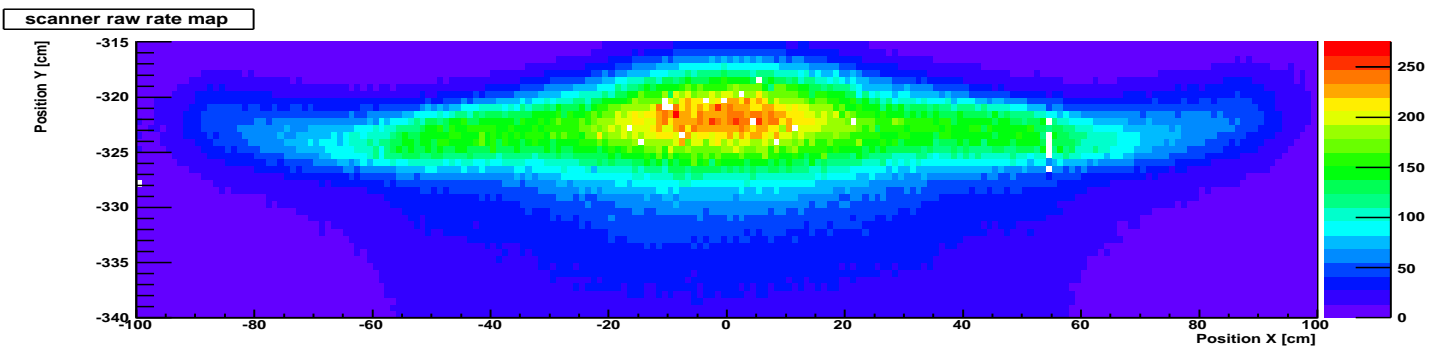

(a) Coincidence rate map

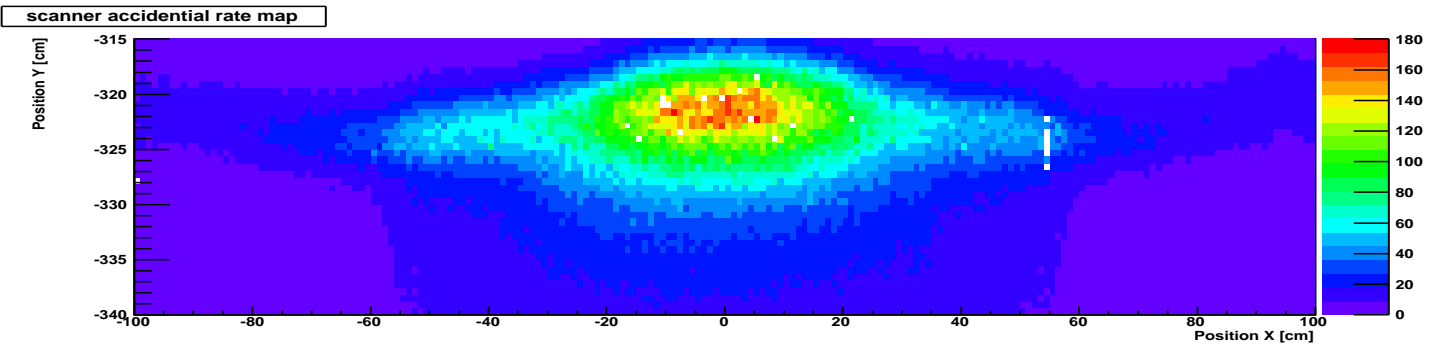

(b) Accidental rate map

Figure 5.1: Raw rate maps of total coincidence and accidental coincidence acquired by the scanner detector at $50 \mu \mathrm{A}$ beam current with the $\mathrm{LH}_{2}$ target (run 6616). In this figure, the $x$ - and $y$-coordinates are in units of $\mathrm{cm}$, and the color scale is the rate in units of $\mathrm{kHz}$. The rate maps are not normalized to beam current, whose distribution has a $0.24 \mu \mathrm{A}$ RMS width during this scan period. The white strips shown in the rate maps are due to beam trips.

measured rate can be removed by normalizing the rate to the instantaneous beam current:

$$
\hat{R}\left(x_{i}, y_{i}\right)=R\left(x_{i}, y_{i}\right) / I_{\text {beam }} \quad\left[\mathrm{Hz} / \mathrm{cm}^{2} / \mu \mathrm{A}\right]
$$

The effects due to beam trips are eliminated by applying beam current cuts. Typically, cuts to reject beam currents of less than $90 \%$ of the nominal values are applied. The normalized rate $\hat{R}\left(x_{i}, y_{i}\right)$ can be used as a characteristic variable for direct comparison of rate distributions in different runs. This is convenient for the scanner detector to observe the stability of the rate distribution and monitor beam optics. However, in order to conveniently perform rate corrections (discussed in Section 5.1.2), most rate maps in this chapter are purposely not normalized to beam current. 


\section{Singles Rate Map}

When the coincidence requirement is not imposed, additional rate is experienced due to single tube backgrounds. This rate is monitored by counting the rates for each PMT separately, which are referred to as the singles rates.

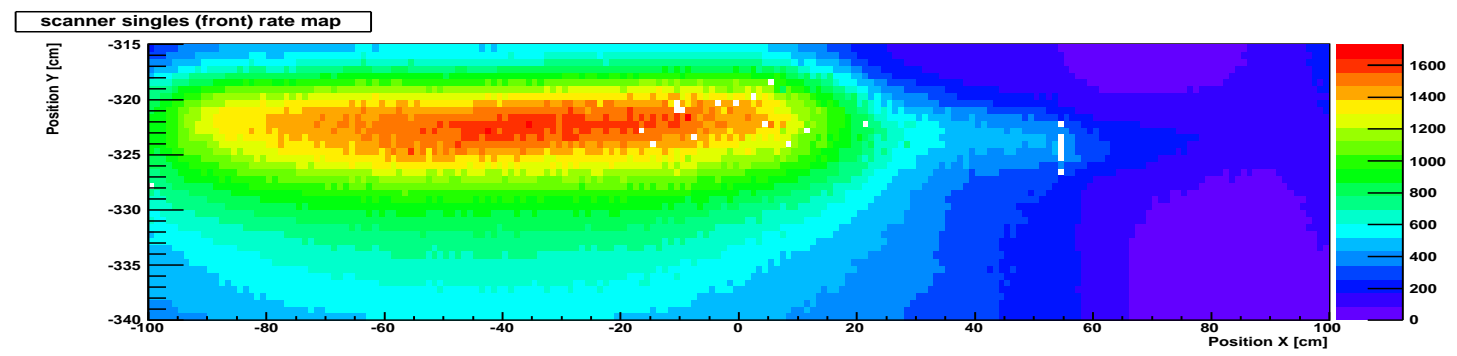

(a) Singles rate map $R_{A}\left(x_{i}, y_{j}\right)$ acquired by the PMT on the right hand side for the front quartz radiator and light guide

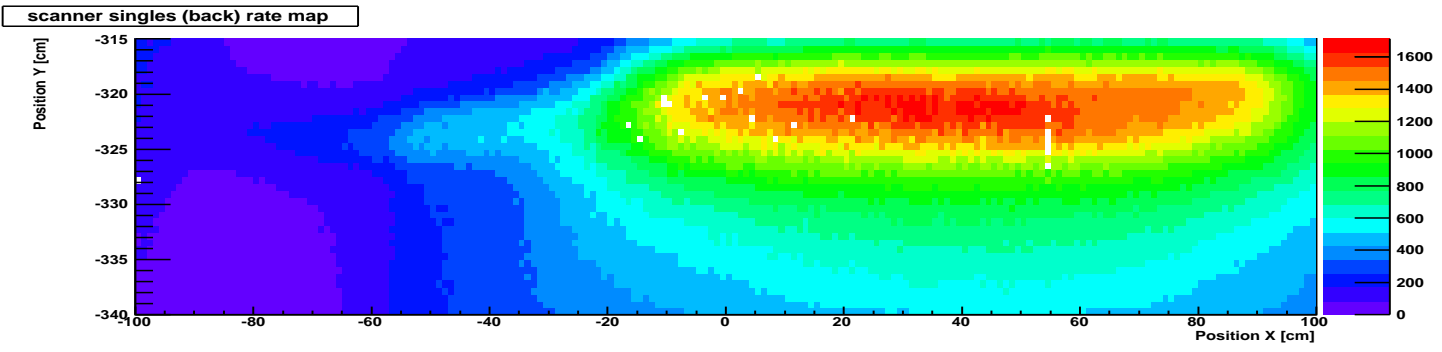

(b) Singles rate map $R_{B}\left(x_{i}, y_{j}\right)$ acquired by the PMT on the left hand side for the back quartz radiator and light guide

Figure 5.2: Singles rate maps (run 6616, at $50 \mu \mathrm{A}$ beam current) acquired by the two PMTs of the scanner detector during a scan at $18.48 \mathrm{~cm}$ upstream of the main detector. The "front" singles (a) refer to the PMT located at the right hand side, and the "back" singles (b) refer to the PMT at the left hand side when looking upstream. The rate maps are not normalized to beam current, whose distribution has a $0.24 \mu \mathrm{A}$ RMS width during this scan period. In this figure, the $x$ - and $y$-coordinates are in units of $\mathrm{cm}$, and the color scale is the rate in units of $\mathrm{kHz}$.

Fig. 5.2 shows the singles rate distributions from run 6616 at a beam current of $50 \mu \mathrm{A}$. The plots show how the two PMT's rates $R_{A}\left(x_{i}, y_{j}\right)$ and $R_{B}\left(x_{i}, y_{j}\right)$ vary with the position of the scanner detector over its scan range. The singles rates are $\sim 10 \times$ larger than the coincidence rates in Fig. 5.1.

Projecting the singles rate map onto the $x$-direction is useful for visualization. The sums of rates along each $y$-bin over a range of $25 \mathrm{~cm}$, the $\mathcal{R}_{A}\left(x_{i}\right)=\sum_{j} R_{A}\left(x_{i}, y_{j}\right)$ and $\mathcal{R}_{B}\left(x_{i}\right)=\sum_{j} R_{B}\left(x_{i}, y_{j}\right)$ for 
each PMT, are shown in Fig. 5.3. The singles rates are the highest when the relevant PMT for that arm itself is close to the center of the main detector on the inner radius side of that octant. Further investigation confirmed that a large number of gamma rays from the Region 1 enclosure and (later installed) beam pipe shielding near the Region 2 area can enter this region and strike the scanner's light guides or PMTs directly (see Fig 5.4). This led to the very high singles rates in each tube. At $50 \mu \mathrm{A}$ beam current, the maximum singles rate was about $1.5 \mathrm{MHz}$.

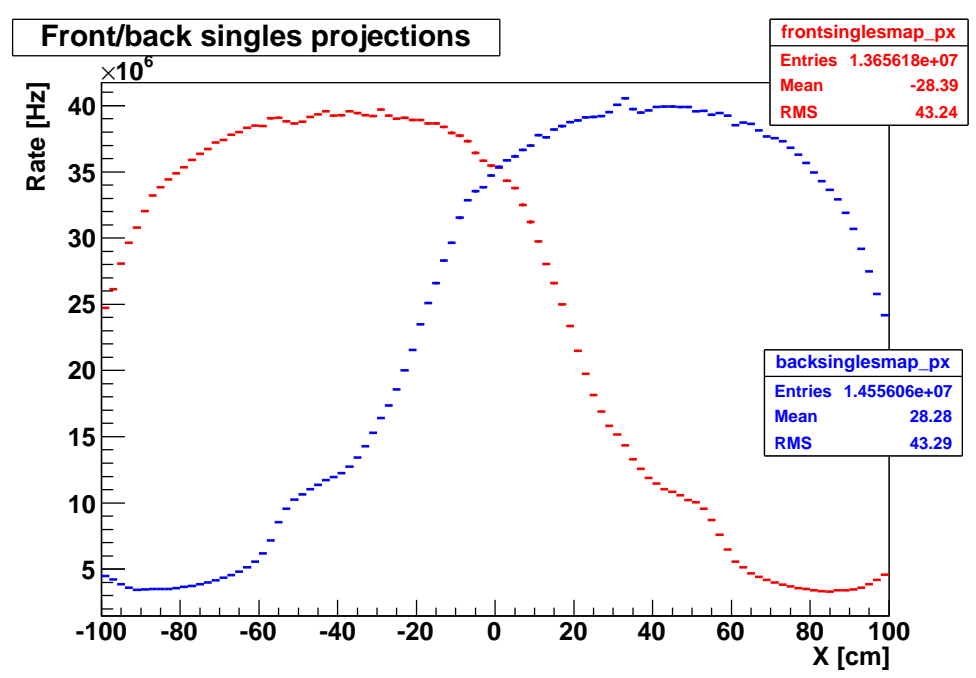

Figure 5.3: Projection of the scanner singles rate maps (in run 6616 at $50 \mu \mathrm{A}$, not normalized to beam current) onto the $x$-axis.

\subsubsection{Rate Corrections}

\section{Correction for Accidental Coincidences}

Accidental coincidences result from uncorrelated events occurring by chance within the coincidence resolving time of the system. If discriminated pulses from one PMT (tube A) have a width $\tau_{1}$, while those from the other PMT (tube B) have a width $\tau_{2}$, and the singles rates in each PMT are $R_{A}$ and 


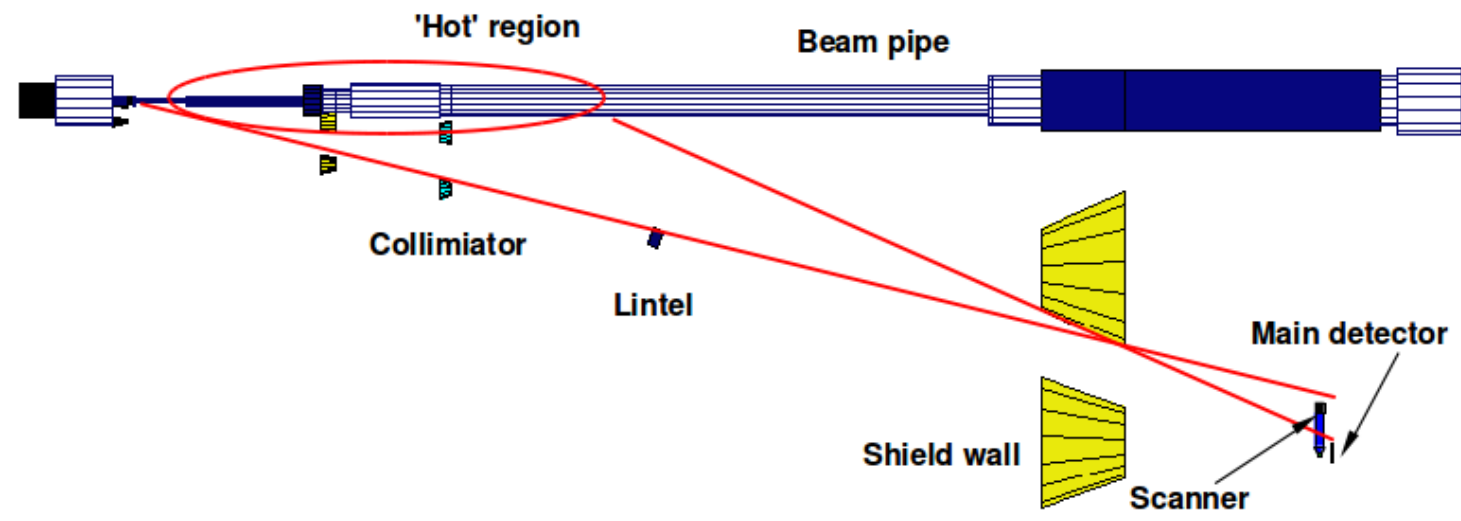

Figure 5.4: Scanner light guides and PMTs are exposed to the gamma rays from interactions in upstream beam pipe and collimators (the 'hot' region, indicated by the red ellipse).

$R_{B}$, then the accidental coincidence rate is [121]

$$
R_{a c c}=R_{A} R_{B}\left(\tau_{1}+\tau_{2}\right)=\tau R_{A} R_{B}
$$

where $\tau=\tau_{1}+\tau_{2}$ is the time window to form a coincidence, which limits the maximum operable rate of the detector. The time window $\tau$ should be as small as possible to reduce accidentals, but large enough to cover the maximum analog pulse widths of the PMT signals themselves. Based on these considerations, $\tau$ was set to $80 \mathrm{~ns}$, with $\tau_{1}=\tau_{2}=40 \mathrm{~ns}$, which is just enough to cover the tail of a large amplitude PMT pulse (the oscilloscope waveforms in Fig. 5.5 show typical PMT pulses), thus avoiding possible double pulsing.

As previously mentioned, a direct measurement of accidental coincidences was conducted using an additional coincidence circuit, with an extra delay line (100 ns) inserted in one PMT channel. The delay was set large enough so that the random accidentals would be outside the true coincidence window.

Neglecting deadtime, the true coincidence rate distribution can be extracted by subtracting the 


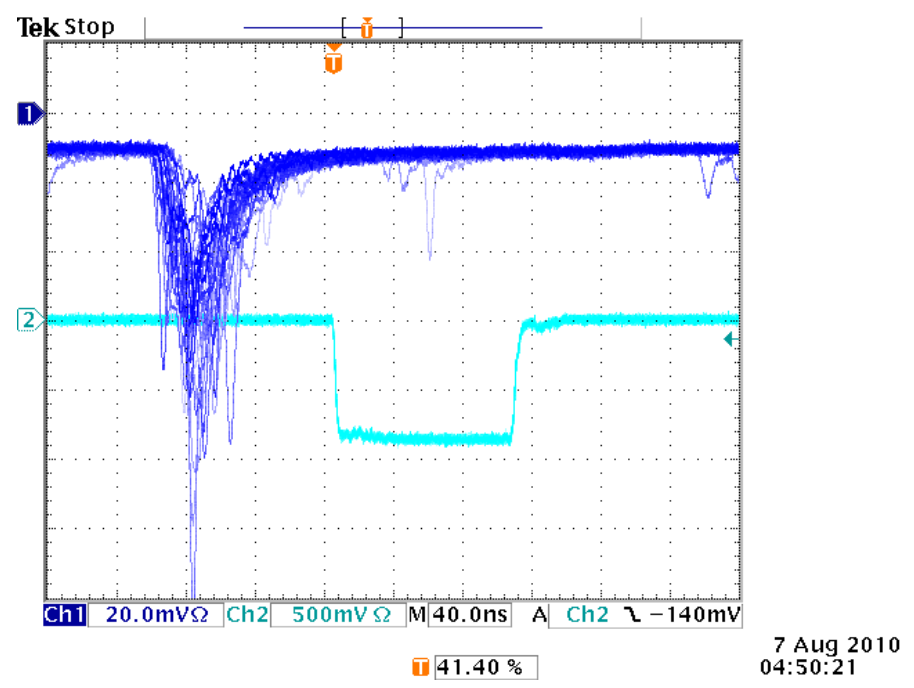

Figure 5.5: Waveforms of typical scanner PMT output pulses (blue) and the delayed charge ADC gate pulse (cyan).

rate of accidental coincidences from the rate of total coincidences, as expressed by

$$
R_{\text {coin }}\left(x_{i}, y_{i}\right)=R_{t o t}\left(x_{i}, y_{i}\right)-R_{a c c}\left(x_{i}, y_{i}\right)
$$

where the $R_{a c c}\left(x_{i}, y_{i}\right)$ are obtained either from direct measurement or calculation based on Eqn. 5.2. The difference between Fig. 5.1 (a) and (b) is calculated using Eqn. 5.3 and displayed in Fig. 5.6. However, this simplified method is only suited to low-current measurements where deadtime losses are low (see Fig. 5.13). As we will see in the next section, this is not the case for operation of the scanner at even higher beam current.

\section{Deadtime Correction}

Deadtime (DT) loss occurs when an incoming pulse is not recorded because either the detector or its electronics chain is busy in processing an earlier event; events occurring within the deadtime period are lost.

For singles measurements, the true singles rates $\left(R_{A}\right.$ and $\left.R_{B}\right)$ can be calculated from the mea- 


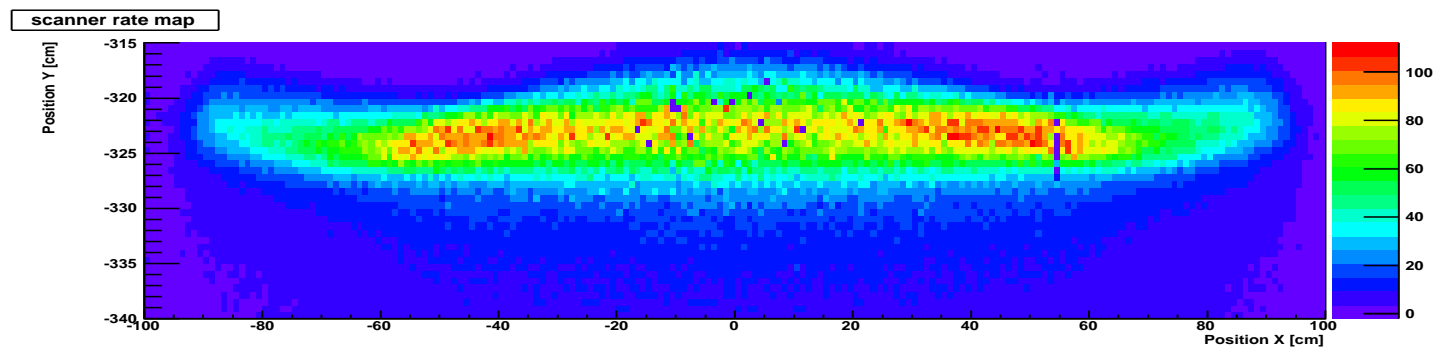

Figure 5.6: Coincidence rate distribution for run 6616 at $50 \mu \mathrm{A}$ beam current, corrected for accidental coincidences as discussed in the text. In the figure, the $x$ - and $y$-coordinates are the Q-weak global coordinates in units of $\mathrm{cm}$. The color scale is the rate in $\mathrm{kHz}$. The rate map is not normalized to beam current, whose distribution has a $0.24 \mu \mathrm{A}$ RMS width during this scan period.

sured singles rates $\left(R_{A}^{\prime}\right.$ and $\left.R_{B}^{\prime}\right)$ by [121]:

$$
R_{A}=\frac{R_{A}^{\prime}}{1-R_{A}^{\prime} \tau_{1}}, \quad R_{B}=\frac{R_{B}^{\prime}}{1-R_{B}^{\prime} \tau_{2}}
$$

where $\tau_{1}$ and $\tau_{2}$ are the deadtimes for channel A and channel B, respectively.

As noted earlier, the accidental coincidence scaler channel records the rate of uncorrelated events in the two PMTs if they occur within the coincidence window. The measured accidental coincidence rate can then be compared to a deadtime corrected version of Eqn. 5.2, i.e:

$$
R_{a c c}=R_{A}^{\prime} R_{B}^{\prime}\left(\tau_{1}+\tau_{2}\right)=\frac{R_{A} R_{B}\left(\tau_{1}+\tau_{2}\right)}{\left(1+R_{A} \tau_{1}\right)\left(1+R_{B} \tau_{2}\right)}
$$

The main coincidence channel records all pulses from two singles channels, counting both the correlated true rate and uncorrelated accidental rate. If $\tau_{1} \leq \tau_{2}$, the total rate in this channel then can be modelled as [122]:

$$
R_{\text {total }}=\frac{R_{\text {coin }}}{1+R_{\text {coin }} \tau_{1}}+\frac{\left(R_{A}-R_{\text {coin }}\right)\left(R_{B}-R_{\text {coin }}\right)\left(\tau_{1}+\tau_{2}\right)}{\left[1+\left(R_{A}-R_{\text {coin }}\right) \tau_{1}\right]\left[1+\left(R_{B}-R_{\text {coin }}\right) \tau_{2}\right]}
$$

where the $R_{\text {coin }}$ is the true coincidence rate. In the case that $R_{\text {coin }} \ll R_{A}$ and $R_{\text {coin }} \ll R_{B}$, the true 
coincidence rate expression can be simplified to

$$
R_{\text {coin }}=\left(R_{\text {total }}-R_{\text {acc }}\right)\left(1+R_{\text {coin }} \tau_{1}\right)
$$

With measured $R_{A}, R_{B}, R_{a c c}$ and $R_{\text {total }}$, the true coincidence rate $R_{\text {coin }}$ can be resolved numerically by applying Newton iterations on Eqn. 5.6. The time resolution and deadtime can be checked by using Eqn. 5.5, where it is assumed that

$$
\tau_{1}=\tau_{2}=\left(\tau_{1}+\tau_{2}\right) / 2=\tau / 2
$$

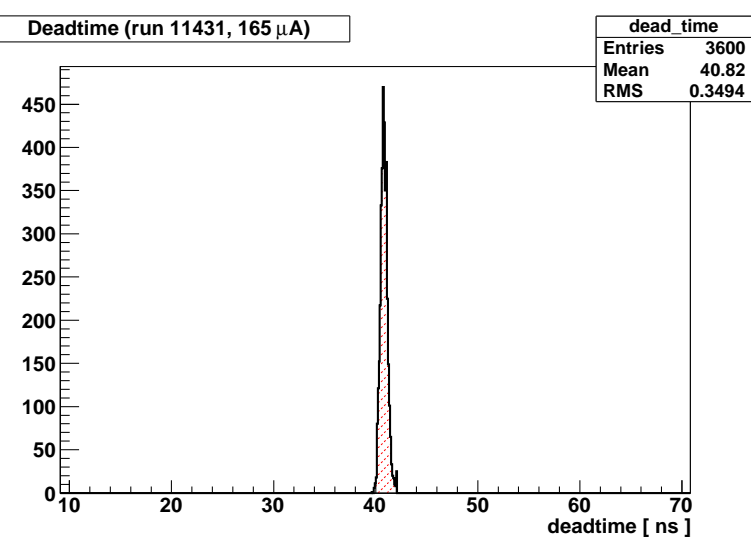

Figure 5.7: Measured deadtime for run 11431 at $165 \mu \mathrm{A}$ beam current with the $\mathrm{LH}_{2}$ target. The mean value of the distribution is $40.82 \mathrm{~ns}$, with a RMS width of $0.35 \mathrm{~ns}$.

As an example of the application of these concepts, the deadtime obtained by applying Eqn. 5.5 and Eqn. 5.8 for run 11431 at $165 \mu \mathrm{A}$ is shown in Fig. 5.7. The deadtime has a value of $40.82 \mathrm{~ns}$ with a RMS width of $0.35 \mathrm{~ns}$. The RMS width was found to vary from run to run when extracted by this technique. We used these measured deadtimes and not the electronics pulse widths to correct the data, since the former were more appropriate to the experimental conditions. The deadtimes found for several scanner runs are listed in Table 5.1. At low beam current, the RMS widths of the deadtime distributions are large, due to small counts in the scaler caused by high triggering rates, as 
will be explained in Section 5.2.3.

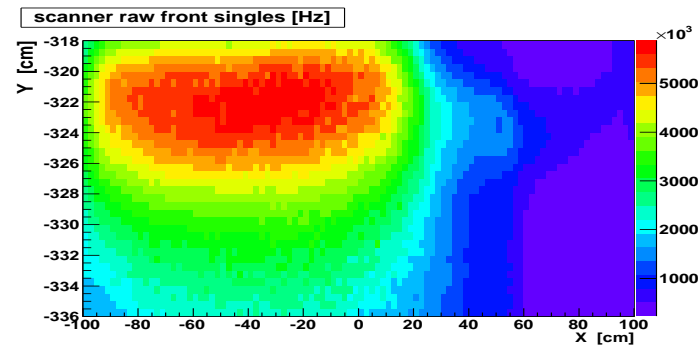

(a) front singles distribution

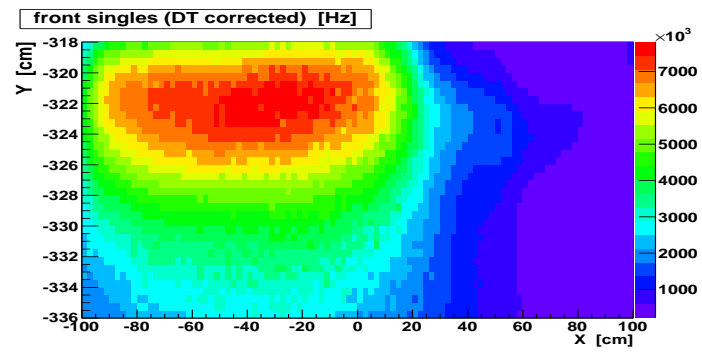

(c) front singles distribution (DT corrected)

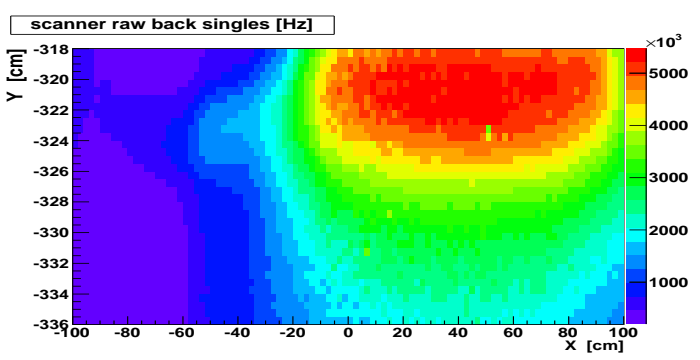

(b) back singles distribution

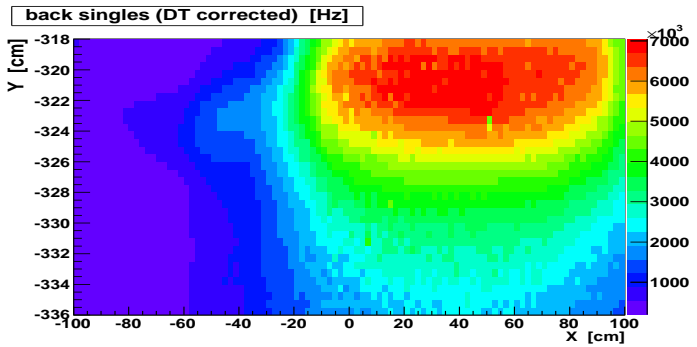

(d) back singles distribution (DT corrected)

Figure 5.8: Deadtime corrections to singles rate distributions for run 10044 at $150 \mu \mathrm{A}$ beam current with the $\mathrm{LH}_{2}$ target. The color scale is the rate in $\mathrm{Hz}$. The rate maps are not normalized to beam current, whose distribution has a $0.21 \mu \mathrm{A}$ RMS width during this scan period.

As another example, the singles rates for run 10044 at $150 \mu \mathrm{A}$ were corrected for deadtime effects using Eqn. 5.4, and the singles distributions before and after deadtime corrections are compared in Fig. 5.8. The comparison (see the color scales) indicates that the maximum correction to the singles rate is $25 \%$. By using the determined deadtime and the corrected singles rates, the true coincidence rate map then can be obtained by applying Eqn. 5.6. The results are shown in Fig. 5.9. The corrected rate map (bottom right plot in Fig. 5.9) and the same map after normalization to instantaneous beam current (see Fig. 5.10 (b)) show a left-right asymmetry. This feature is discussed in Section 5.1.3.

The above correction method was tested by performing a PMT discriminator pulse width scan. At several different discriminator pulse width settings, the deadtime was obtained by using Eqn. 5.5, 

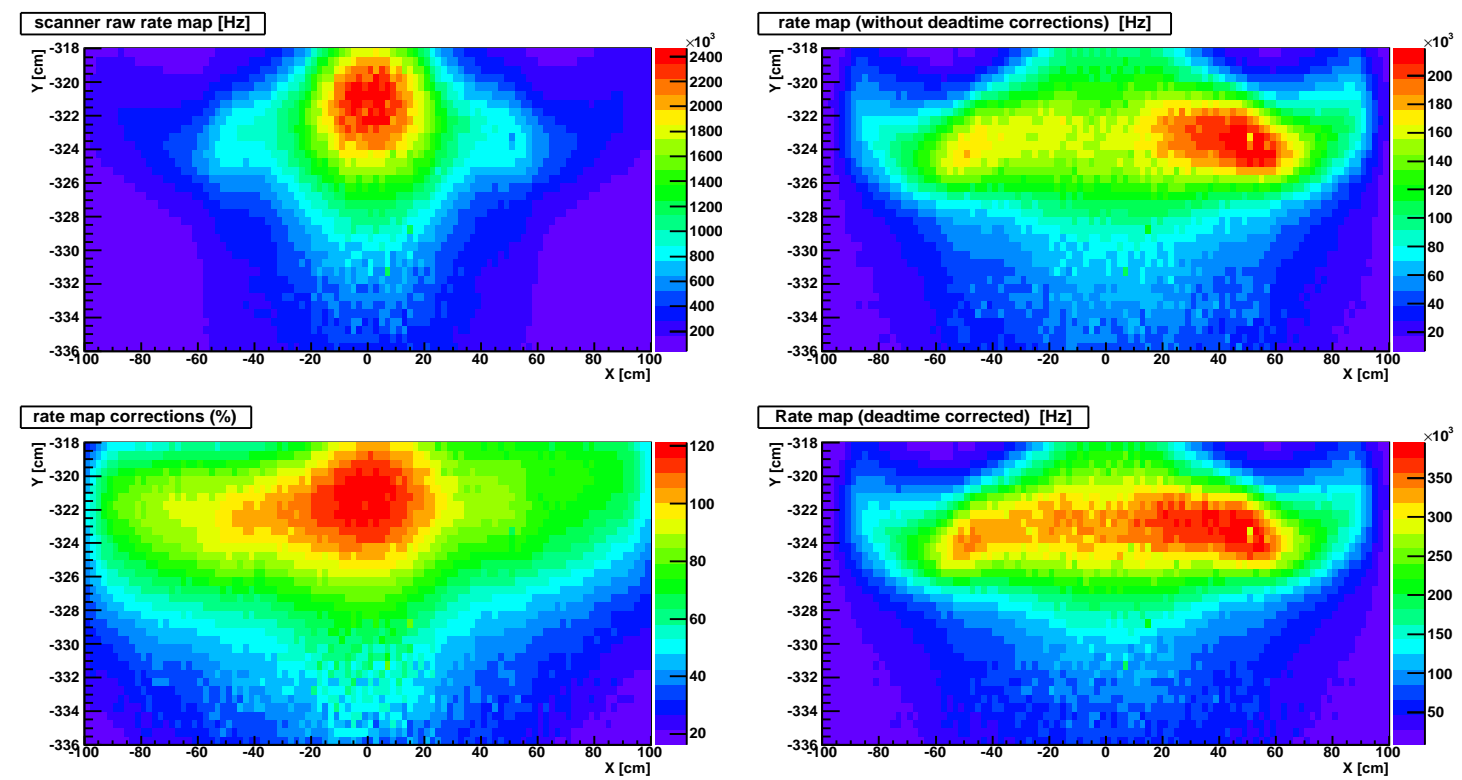

Figure 5.9: Deadtime corrections to the scanner rate map. The top left image is the raw rate map measuring the total coincidence, the top right image is rate map with accidentals subtracted, the bottom left image shows the distribution of deadtime correction effects expressed in percent, and the bottom right image is rate map after both accidental coincidence and deadtime corrections for run 10044 at $150 \mu \mathrm{A}$ with the $\mathrm{LH}_{2}$ target. The rate maps are not normalized to beam current, whose distribution has a $0.21 \mu \mathrm{A}$ RMS width during this scan period.

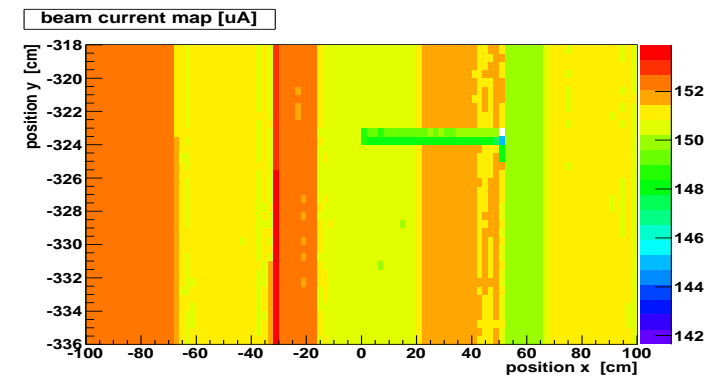

(a)

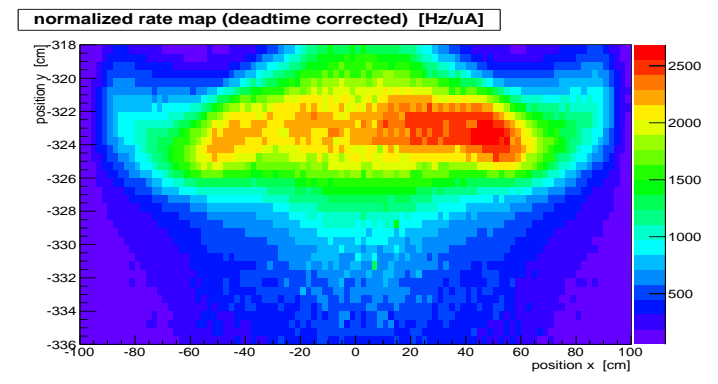

(b)

Figure 5.10: (a) The beam current map during the scanner scan period in run 10044. (b) The normalized scanner rate map (the bottom right plot in Fig. 5.9 was normalized to instantaneous beam current) in run 10044 at $150 \mu \mathrm{A}$. A beam current cut was applied in this analysis so that only data with $I_{\text {beam }}>140 \mu \mathrm{A}$ were used. 
and the results are shown in Fig 5.12. The deadtimes determined by this method were slightly smaller than the discriminator pulse width settings. This is believed to be caused by two additional inverting gates in the accidental coincidence channel, which tend to reduce the discriminated pulse widths by about $1-3 \mathrm{~ns}$.

\begin{tabular}{cccccccc}
\hline \hline $\begin{array}{c}\text { Run } \\
\text { Number }\end{array}$ & $\begin{array}{c}\text { Beam } \\
{[\mu \mathrm{A}]}\end{array}$ & $\begin{array}{c}\text { Disc. Pulse } \\
\text { Width }[\mathrm{ns}]\end{array}$ & $\begin{array}{c}\text { Deadtime } \\
{[\mathrm{ns}]}\end{array}$ & $\begin{array}{c}\text { DT RMS } \\
\text { Width }[\mathrm{ns}]\end{array}$ & $\begin{array}{c}\text { Left Bin } \\
{[\mathrm{kHz} / \mu \mathrm{A}]}\end{array}$ & $\begin{array}{c}\text { Central Bin } \\
{[\mathrm{kHz} / \mu \mathrm{A}]}\end{array}$ & $\begin{array}{c}\text { Right Bin } \\
{[\mathrm{kHz} / \mu \mathrm{A}]}\end{array}$ \\
\hline 6615 & 10 & 40 & 40.9 & 7.7 & 1.74 & 1.77 & 2.14 \\
6616 & 50 & 40 & 39.5 & 2.1 & 1.72 & 1.84 & 2.05 \\
9710 & 145 & 40 & 42.9 & 0.9 & 1.16 & 1.77 & 2.18 \\
10044 & 150 & 40 & 42.8 & 1.4 & 1.79 & 1.80 & 2.41 \\
10987 & 150 & 20 & 17.6 & 0.4 & 1.78 & 2.04 & 2.29 \\
10913 & 150 & 10 & 9.2 & 0.2 & 1.75 & 1.84 & 2.16 \\
$10988-1$ & 150 & 30 & 29.0 & 0.4 & 1.76 & 1.96 & 2.29 \\
$10988-2$ & 150 & 40 & 37.1 & 0.4 & 1.81 & 1.89 & 2.29 \\
10991 & 150 & 50 & 46.0 & 0.7 & 1.92 & 1.92 & 2.24 \\
11431 & 165 & 45 & 40.8 & 0.4 & 1.77 & 1.87 & 2.29 \\
\hline Mean & & & & 1.72 & 1.87 & 2.23 \\
RMS & & & & 0.19 & 0.08 & 0.10 \\
\hline \hline
\end{tabular}

Table 5.1: Comparison of total rate corrections at three typical positions, the left bin $(-50 \mathrm{~cm}$, $-322.5 \mathrm{~cm})$, central bin $(0,-322.5 \mathrm{~cm})$ and right bin $(50 \mathrm{~cm},-322.5 \mathrm{~cm})$, in the rate distribution for each run (see Fig. 5.11 for the bin locations). 'RMS' in the table is the ' $\sigma$ ' of the deadtime distribution.

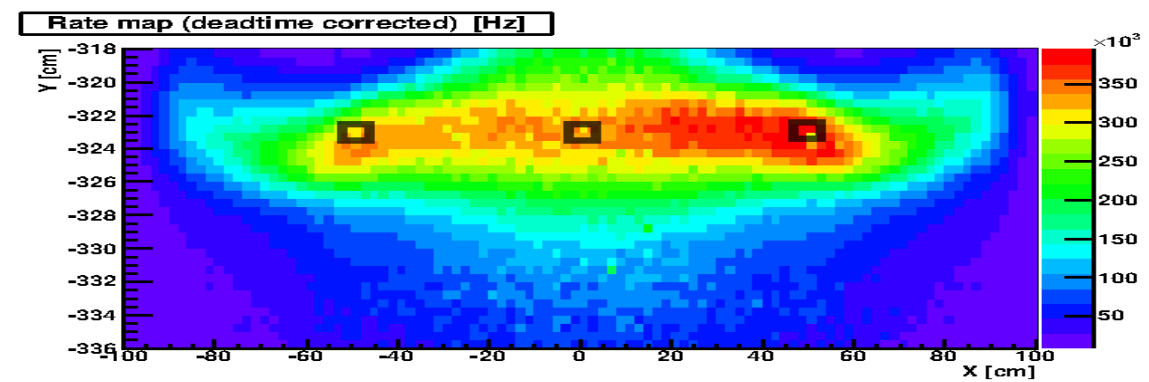

Figure 5.11: Locations of the left bin $(-50 \mathrm{~cm},-322.5 \mathrm{~cm})$, central bin $(0,-322.5 \mathrm{~cm})$ and right bin $(50 \mathrm{~cm},-322.5 \mathrm{~cm})$ are indicated by three black boxes in a rate map. These three typical positions are in the highest rate region of the Q-weak elastic electron image. 


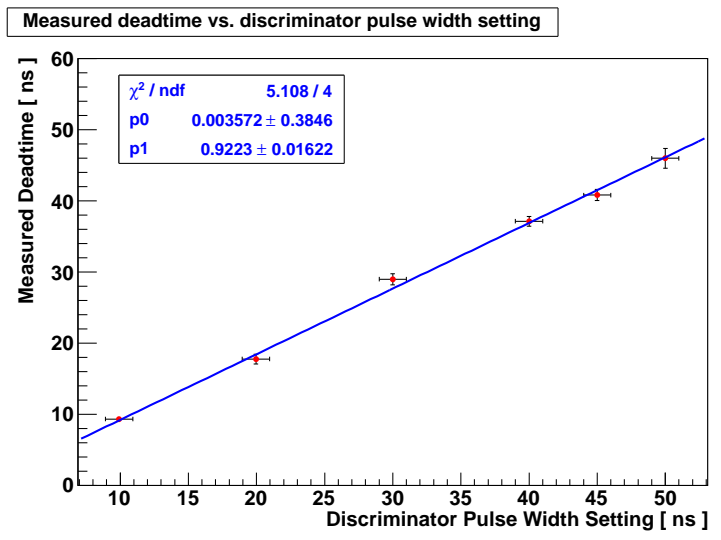

Figure 5.12: Measured deadtime versus the discriminator output pulse width settings in the scanner pulse width scan. The discriminator output pulse widths are measured with an oscilloscope, so an approximate $1 \mathrm{~ns}$ error bar is imposed. The size of the $y$-error-bar is $2 \sigma$ of the measured deadtime.

After making deadtime corrections, the rate maps tend to show improved linearity with beam current. An example can be seen in Fig. 5.13, which shows comparisons of rates before and after corrections for several runs at different beam currents. The data points in this figure were taken from run 6615 , run 6616, run 9710, run 10044 and run 11431 , with beam currents $10-165 \mu \mathrm{A}$, as listed in Table 5.1. All except run 11431 had discriminator pulse width of $40 \mathrm{~ns}$; run 11431 had discriminator pulse width of $45 \mathrm{~ns}$. The rates at three typical positions, the left, central and right bins, of each rate map are displayed (see Fig. 5.11 for the bin locations). The top panels in this figure show the front singles rates in three bins, with the rates before and after DT corrections being represented by blue and red points (or lines), respectively. Similarly, the middle panels show the rates for back singles. It can be seen that larger DT correction effects appear in the left bin for front singles, while in the right bin for back singles. The corrected front singles rates indicate obvious non-linearity with beam current. However, the coincidence rates after DT corrections demonstrate good linearity in all three bins, which are shown as the red lines in the bottom panels. The other two lines, the green one and the blue one, represent the raw total coincidence rates and those after accidental coincidence subtractions. From the three bottom plots, the trend can be seen that accidental corrections and DT 


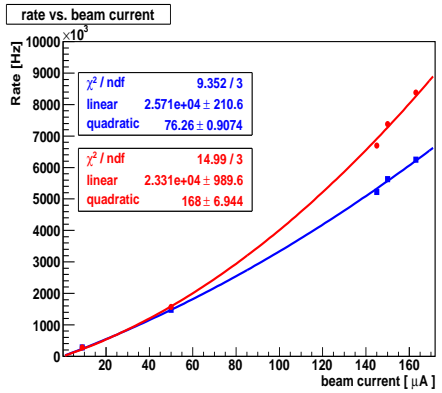

(a) front PMT singles, left bin

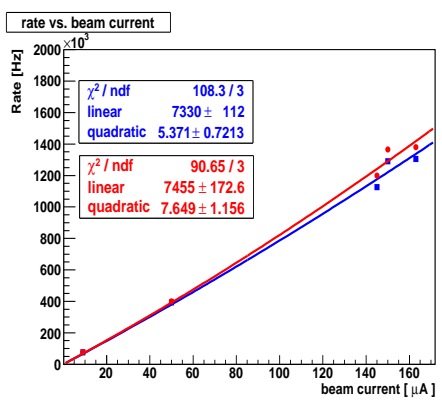

(d) back PMT singles, left bin

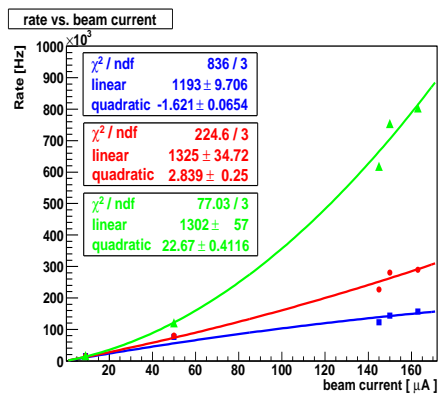

(g) coincidence, left bin

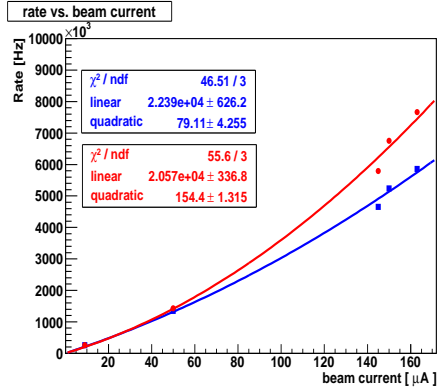

(b) front PMT singles, central bin

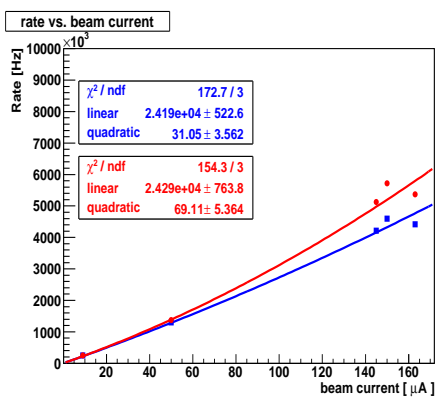

(e) back PMT singles, central bin

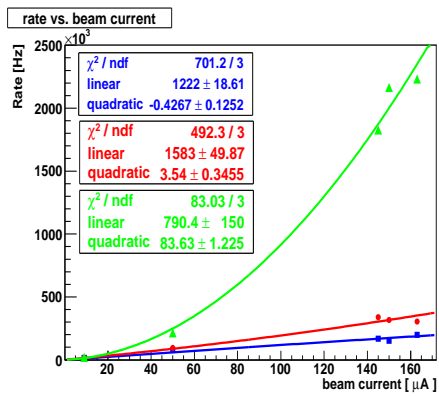

(h) coincidence, central bin

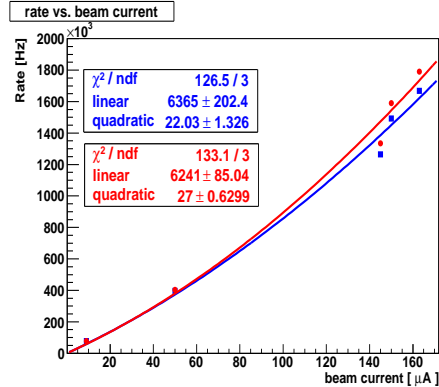

(c) front PMT singles, right bin

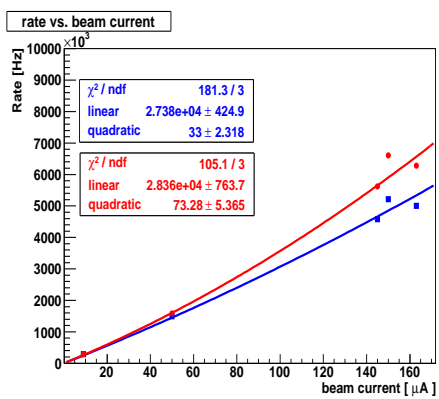

(f) back PMT singles, right bin

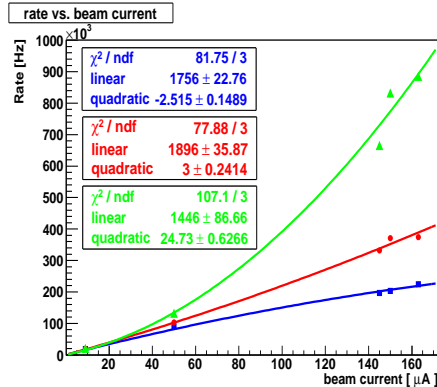

(i) coincidence, right bin

Figure 5.13: Singles and coincidence rate corrections for the left bin $(-50 \mathrm{~cm},-322.5 \mathrm{~cm})$, central bin $(0,-322.5 \mathrm{~cm})$ and right bin $(50 \mathrm{~cm},-322.5 \mathrm{~cm})$ of the scanner rate maps (see Fig. 5.11 for the bin locations). The raw total coincidence rates are indicated by the green curves; the raw singles rates and the rate differences between total coincidence rates and accidental rates are indicated by the blue curves; the rates after deadtime corrections are indicated by the red curves. The error bar on each data point was assigned by adding $1 \mathrm{~ns}$ to its deadtime. Each set of data points was fitted to function $f(x)=a x+b x^{2}$, where $a$ and $b$ are linear and quadratic parameters, respectively. 
corrections affect the coincidence rates with opposition sign. The plots (g) - (i) in Fig. 5.13 also imply that the correction method is able to recover the true rate information. However, there are still non-negligible quadratic terms in the fits, which might be caused by rate-dependent PMT gain shifts, which will require further investigation.

\subsubsection{Scanner Results Discussion}

Fig. 5.14 shows a comparison of two runs taken at $10 \mu \mathrm{A}$ and $50 \mu \mathrm{A}$ beam currents, respectively. The measured rate distributions in these two runs are symmetric and show good linearity with beam current (i.e. the normalized rates at $10 \mu \mathrm{A}$ are approximately the same as these at $50 \mu \mathrm{A}$ ). The relative residual (the difference over the average) of the normalized rate distribution $\hat{R}\left(x_{i}, y_{j}\right)$ between them is about $0.86 \%$. Both singles rates also show linear response to beam current. In the $10 \mu \mathrm{A}$ run, the maximum singles rate is $0.3 \mathrm{MHz}$, while in the $50 \mu \mathrm{A}$ run, it increases by a factor of 5 , as expected.

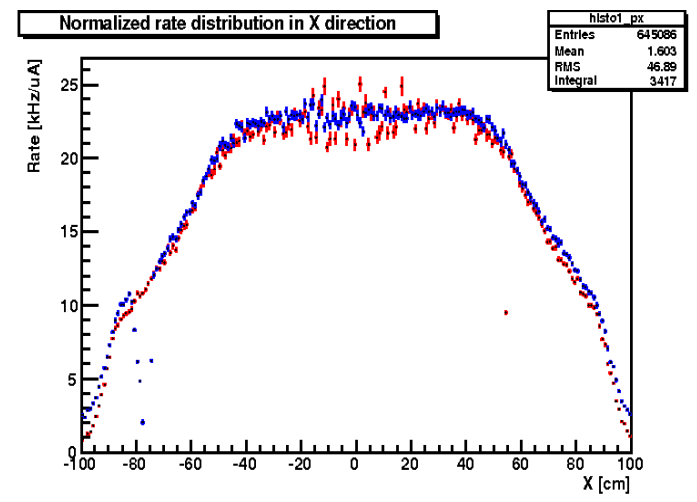

(a) Projection of rate distribution in the $x$-direction

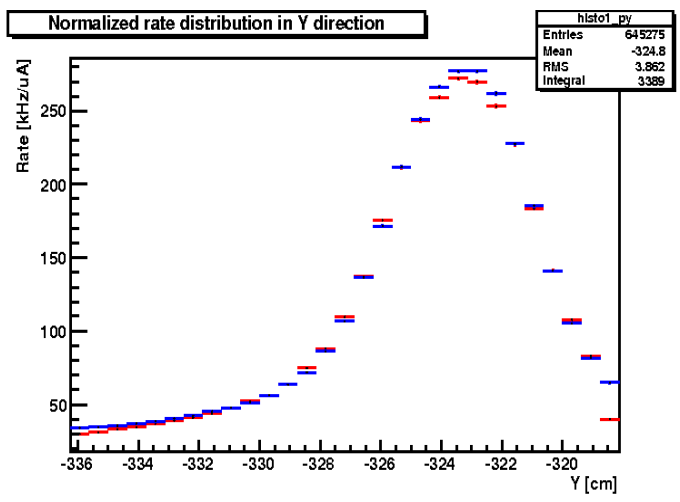

(b) Projection of rate distribution in the $y$-direction

Figure 5.14: Comparison of normalized rate distribution projections at $10 \mu \mathrm{A}$ (blue, run 6615) and $50 \mu \mathrm{A}$ (red, run 6616) in $x$ (horizontal) and $y$ (vertical) directions. The position information for these runs was recorded by a less accurate 12-bit QDC, leading to fluctuations in the rate maps, which are particularly obvious in figure (a).

However, analyzing later runs taken in February 2011 (once higher beam currents $(\gtrsim 150 \mu \mathrm{A})$ were routinely delivered to the experiment), it was found that the singles rates for the front PMT 
increased by $33 \%$ over expectation at $145 \mu \mathrm{A}$. The back PMT singles rate changed very little. Not unexpectedly, very high accidental coincidence rates were found near the central region, which dominated the total coincidence rate even more than at $50 \mu \mathrm{A}$. The maximum accidental coincidence rate was $90 \%$ of the total coincidence rate at $165 \mu \mathrm{A}$. The rate distribution images measured by the scanner also showed a slight left-right asymmetric trend (see Fig. 5.10 (b)).

The reason for the changes in the front singles and the rate distributions have not been clearly identified. Here we suggest some possible explanations which merit further study. The singles rate increase in the front PMT could be caused by the following:

- It occurred since the first high beam current run, which was taken right after the installation of new Region 2 shielding. The shielding change may have led to increased gamma backgrounds for the front PMT.

- It may be caused by a gain shift or pedestal shift in the PMT itself due to large anode current, which would shift events above the threshold of the discriminator. At $150 \mu \mathrm{A}$, the maximum singles rate for the front tube is $\sim 5.2 \mathrm{MHz}$, higher than the expected $4.5 \mathrm{MHz}$ (linearly scaled from lower current runs). At $1500 \mathrm{~V}$ bias voltage, the PMT anode current measured with a picoammeter is $\sim 10 \mu \mathrm{A} / \mathrm{MHz}$, which agrees with the calculation:

$$
\begin{aligned}
\text { Anode Current } & =\# \text { of PE } \times \text { PMT Gain } \times \text { Electron's Charge } \times \text { Event Rate } \\
& =(10 \mathrm{PE}) \times\left(6 \times 10^{6}\right) \times\left(1.6 \times 10^{-19} \mathrm{C}\right) \times(1 \mathrm{MHz}) \simeq 10 \mu \mathrm{A} .
\end{aligned}
$$

With $\sim 5 \mathrm{MHz}$ singles rate, the PMT anode current $\sim 50 \mu \mathrm{A}$ is still within the PMT's operational limit (maximum continuous anode current $200 \mu \mathrm{A}$ ), but close to the PMT voltage divider's limit $(56 \mu \mathrm{A})$ for linear operation. The linearity of the PMT is affected by more than $4 \%$ due to the limitation of the divider circuitry once the PMT anode current goes beyond the limit (see the characteristics of Photonis S563 voltage divider in Ref. [123]).

- It might also arise from another unknown electronics systematic effect at high rate. 
In addition to the singles rate, there is also the mystery of the left-right asymmetric trend in the measured rate distributions in high beam current runs. There is some possibility that the left-right asymmetry is a real physics effect representing a new background in the main detector, and possibly having arisen in the Region 2 shielding change. Indeed Region 3 tracking data seem also to see a slight asymmetry in the bottom octant (see Fig. 5.15, and Table 5.2, which summarizes the leftright asymmetries of rate distributions measured by the scanner or tracking detectors). The other possibility would be an imperfect deadtime (or accidental coincidence) correction, with the front PMT possibly experiencing an increased singles rate. This idea also has support, because Fig. 5.9 shows that the degree of left-right symmetry improved when deadtime corrections were taken into account.

Based on Run I scanner data analysis, the following suggestions are made for possible improvements to the system for Q-weak Run II:

- Installation of good shielding for the scanner PMTs is the most effective way to improve detector performance. This will significantly reduce the total rate received by the detector, avoid large PMT anode current and non-linearity caused by background gamma rays, and improve the signal-to-background ratio. This is possible with several feet of concrete or steel, but could be very expensive. It is also a difficult problem because smaller radiation shielding could even increase the backgrounds due to EM showering.

- Installation of a lead pre-radiator for the scanner quartz elements is recommended, as well as reduction of the PMT gains to adapt to the resultant larger signal. This will help to increase the signal-to-background ratio because the yields from backgrounds would be reduced by dropping the gain of the PMTs. Similar effects can be achieved by moving the scanner detector downstream of the main detector since there is already a pre-radiator installed in front of the main detector. This suggestion is being pursued at the time of writing.

- Before Run II data taking, it would be advisable to perform linearity tests at high rates on the scanner PMTs themselves. 
- The randoms measurement should be rechecked. Each setting in the electronics system should be carefully measured, such as the digital pulse-widths out of the PMT discriminators and other logic modules, and the deadtime should also be measured directly (using e.g. a highfrequency $20 \mathrm{MHz}$ random pulser).

- Monte Carlo modelling of deadtime and accidental coincidences should be carried out, to confirm the techniques used for rate corrections.

- A better understanding of backgrounds could be obtained by performing a background scan with the scanner's quartz radiators removed from the detector.

- A logic circuit could be added to the electronics chain to narrow the width of the coincidence window so as to reduce the accidental coincidences. The suggested method is to form narrow ( $\sim 5$ ns) secondary pulses after the 40 ns wide discriminator pulses. Those narrowed pulses would then be fed to the coincidence unit.

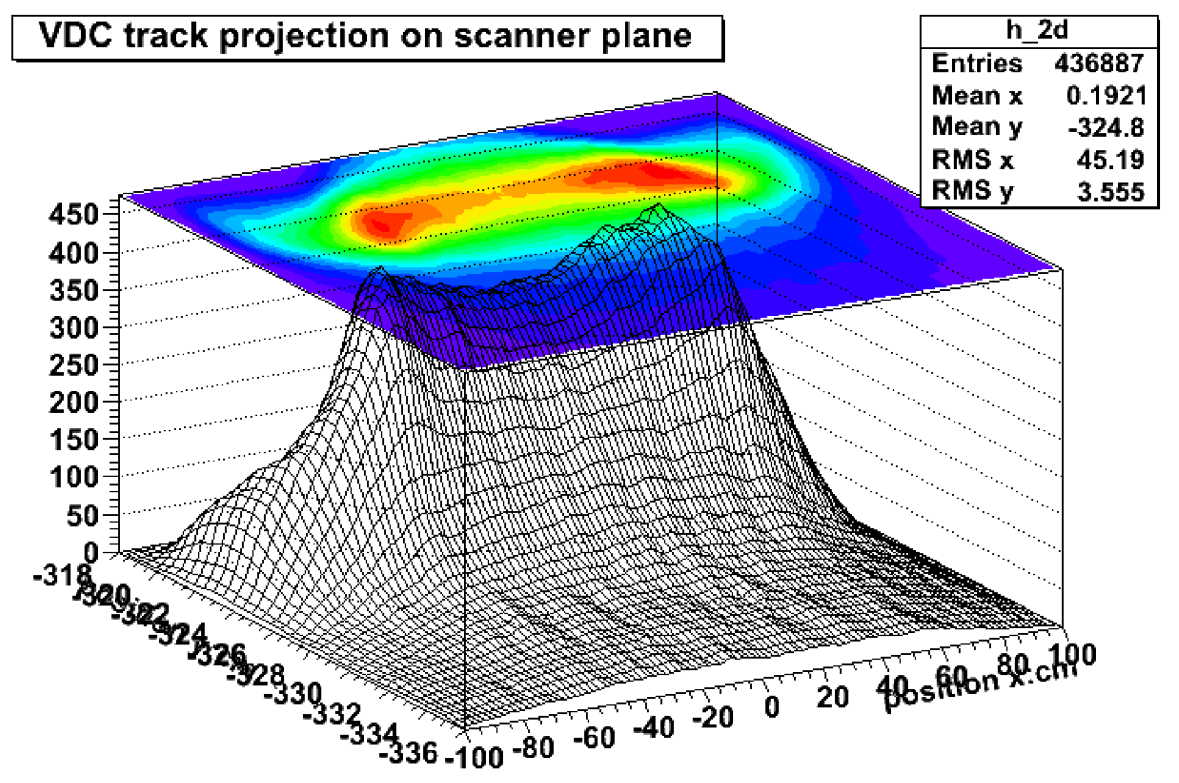

Figure 5.15: The VDCs track projection onto the scanner plane for run 8672 at $25 \mathrm{nA}$ in octant 7 (bottom octant). 


\subsection{Momentum Transfer Extrapolation}

As discussed earlier, the scanner detector was calibrated against the tracking chambers in Region 3 at beam currents less than $100 \mathrm{nA}$. The rate distribution in parity mode was measured at 150 $180 \mu \mathrm{A}$. This section discusses the comparison of low current and high current scans to extrapolate the light-weighted $\left\langle Q^{2}\right\rangle$ to high current.

\subsubsection{Calibration to Region 3 VDCs}

The scanner detector's response to electrons is expected to be uniform with scan position despite the complicated geometry involved, based on Monte Carlo simulations (see Fig. 3.18). This uniformity can in principle be measured by running the Region 3 tracking chambers and the scanner together in dedicated low current tracking runs. The VDCs can provide information on each track in the zero magnetic field region close to the scanner detector location. By correlating each partial track with a main detector hit and light yield, the elastic event flux in the focal plane can be determined. The determined rate, $R_{V D C}\left(x_{i}, y_{j}\right)$, in the scanner plane can be treated as the true rate for calibration of the scanner. A calibration factor map

$$
\beta\left(x_{i}, y_{j}\right)=\frac{R_{V D C}\left(x_{i}, y_{j}\right)}{R_{\text {cal. }}\left(x_{i}, y_{j}\right)}
$$

can then be generated by obtaining scanner measurements $R_{c a l}\left(x_{i}, y_{j}\right)$ (taking data with the VDCs in the same run) at all points over the full scan range. As long as the scanner configuration is unchanged, the actual rate distribution, $R\left(x_{i}, y_{j}\right)$, in other higher current runs can be recovered using the scanner measurements $R_{\text {meas }}\left(x_{i}, y_{j}\right)$ (with proper corrections) and the calibration factor map $\beta\left(x_{i}, y_{j}\right)$, i.e:

$$
R\left(x_{i}, y_{j}\right)=\beta\left(x_{i}, y_{j}\right) R_{\text {meas }}\left(x_{i}, y_{j}\right)
$$

Due to limited beam time, the scanner was calibrated in this fashion at only a few positions during Q-weak Run I. Fig. 5.16 gives an example, showing the calibration at a fixed scanner location 
in event mode in run 8668 at $25 \mathrm{nA}$ beam current. During data taking in this run, the scanner detector was parked with its sensitive elements centered at the global coordinates $(0,-323 \mathrm{~cm})$. Region 3 partial tracks were reconstructed and projected onto the scanning plane within an area $1 \times 1 \mathrm{~cm}^{2}$ centered at the scanner location (see Fig. 5.16 (a)). While the scanner orientation is at $45^{\circ}$ to the $1 \mathrm{~cm}^{2}$ VDC event box used to compare scanner and VDC rates (see Fig. 3.4), assuming a relatively uniform event distribution, the systematic error due to these different orientations is smaller than other uncertainties in the calibration. Assuming a 100\% VDC chamber efficiency, by comparing the hits from those tracks and the corresponding hits measured by the scanner detector directly (see Fig. 5.16 (b)), the calibration factor at this position was measured to be $1.40 \pm 0.05$, where the uncertainty is simply estimated as the standard deviation $\sigma$ of a few measurements. This means the scanner has an efficiency of $1 / 1.4(\sim 70 \%)$ relative to its nominal $1 \times 1 \mathrm{~cm}^{2}$ active area, at $\sim 3 \mathrm{PE}$ threshold setting. This result is reasonable, based on a Monte Carlo simulation taking into account the detailed detector geometry (see Fig. 3.18).

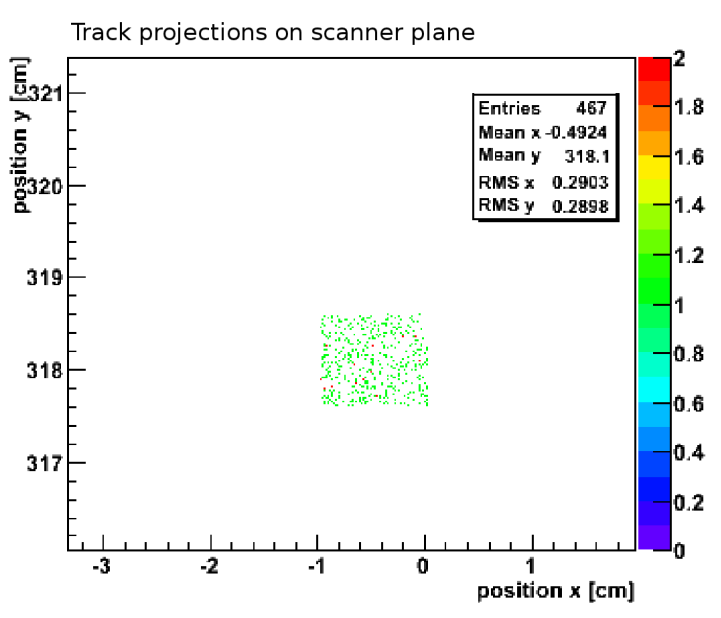

(a)

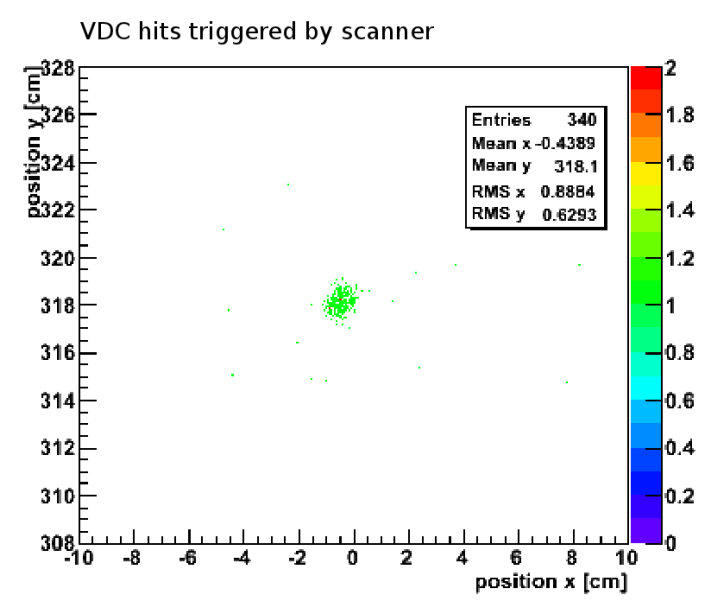

(b)

Figure 5.16: Scanner calibration to VDCs at a fixed scanner position in run 8668 at $25 \mathrm{nA}$ beam current: (a) The hits within a unit area $\left(1 \mathrm{~cm}^{2}\right)$ measured by the VDCs centered on the scanner probe position. (b) The VDC hit distribution for scanner events over 3 PE threshold. In this figure, some events extrapolate far from the scanner due to poor track reconstruction. The calibration factor is determined as the ratio of hits, i.e, $467 / 340$. 
This calibration method is useful for the scanner to obtain the actual rate for charged particles. For the purpose of $Q^{2}$ extrapolation, a relative calibration was devised, which does not require an absolute rate measurement, as discussed below.

\subsubsection{Dimensionless Normalization}

Ideally, we should obtain a calibration factor map over the entire scan region in dedicated calibration runs, which would involve both the scanner and the VDCs. During Q-weak Run I, however, such data were not acquired. Instead, two independent runs were compared: one is an event mode VDC run at a beam current of $25 \mathrm{nA}$; another is a scanner run at a higher beam current.

To make the comparison between the two runs, VDC partial tracks were propagated to the scanner plane, and the number of hits per unit area $\left(1 \mathrm{~cm}^{2}\right)$ per unit time $R_{3}^{\prime}\left(x_{i}, y_{j}\right)$ was calculated. This VDC rate distribution was then normalized to the central bin $\left(R_{0}^{3}\right)$ as $R_{3}^{\prime}\left(x_{i}, y_{j}\right) / R_{0}^{3}$, which reflects the relative hit distribution over the effective scan area. For the scanner detector, the rate distribution is expressed in a similar way. The relative scanner rate distribution is $\hat{R}_{s}^{\prime}\left(x_{i}, y_{j}\right) / \hat{R}_{0}^{s}$, where $\hat{R}_{s}^{\prime}\left(x_{i}, y_{j}\right)$ represents the beam-current-normalized rate in one bin (corrected for deadtime and accidentals, as discussed in Section 5.1), and $\hat{R}_{0}^{s}$ represents the beam current normalized rate in the central bin.

Normalizing to the respective total VDC hits or total scanner rate produced similar results. In this way, the VDC hit map and the scanner rate map are transformed to two dimensionless maps, making them easier to compare, and making the comparison wholly dependent on image shapes in the focal plane, as opposed to normalization issues.

The normalized VDC hit and scanner rate maps reflect the geometric distribution of the scattered electron flux. A direct comparison between them will generate a relative calibration factor map. Assuming that the central bin content of the scanner detector has perfect linearity to beam currents from $25 \mathrm{nA}$ to $10 \mu \mathrm{A}$ (the lowest beam current for the full bar scanner run so far) and that the single point calibration technique is accurate, this relative calibration factor map can be used to deduce the absolute rate distribution at $10 \mu \mathrm{A}$ or higher beam currents. 


\subsubsection{Contrast}

By comparing the relative hit distribution measured by the VDCs and the relative rate distribution measured by the scanner from bin to bin over the full scanning range, the difference between them at any position can be obtained, which is described by

$$
\operatorname{Residual}\left(x_{i}, y_{j}\right)=\frac{\hat{R}_{s}^{\prime}\left(x_{i}, y_{j}\right)}{\hat{R}_{0}^{s}}-\frac{R_{3}^{\prime}\left(x_{i}, y_{j}\right)}{R_{0}^{3}} \text {. }
$$

For convenience, we define a variable "contrast", or $C$. The contrast in one bin at beam current $I_{l}$ for the $l$-th measurement, as described by $C\left(I_{l}, x_{i}, y_{j}\right)$, can be expressed in terms of the $\operatorname{Residual}\left(x_{i}, y_{j}\right)$, or the ratio of two fluxes as:

$$
C\left(I_{l}, x_{i}, y_{j}\right)=1+\frac{\operatorname{Residual}\left(x_{i}, y_{j}\right)}{\frac{R_{3}^{\prime}\left(x_{i}, y_{j}\right)}{R_{0}^{3}}}=\frac{\frac{\hat{R}_{s}^{\prime}\left(x_{i}, y_{j}\right)}{\hat{R}_{0}^{s}}}{\frac{R_{3}^{\prime}\left(x_{i}, y_{j}\right)}{R_{0}^{3}}} .
$$

To describe the contrast over the entire effective area in a total of $k$ bins $^{2}$, one can use two average variables: the mean contrast, given by:

$$
C\left(I_{l}\right)=\frac{\sum_{i=1}^{m} \sum_{j=1}^{n} C\left(I_{l}, x_{i}, y_{j}\right)}{k}
$$

and the total-rate ratio, given by:

$$
\frac{R^{\prime}}{R_{0}}=\frac{\sum_{i=1}^{m} \sum_{j=1}^{n} C\left(I_{l}, x_{i}, y_{j}\right) R_{3}^{\prime}\left(x_{i}, y_{j}\right)}{\sum_{i=1}^{m} \sum_{j=1}^{n} R_{3}^{\prime}\left(x_{i}, y_{j}\right)}
$$

where $m$ and $n$ are the maximum number of bins in $x$ - and $y$-directions, respectively, and the total bins $k=m \times n$. The mean contrast $C\left(I_{l}\right)$ reflects the average difference between the scanner's rate map relative to the VDC's map over the entire area at beam current $I_{l}$, and the total-rate ratio contains the rate distribution information at high beam current and reflects the contrast of the total

\footnotetext{
${ }^{2}$ For the full area of $200 \mathrm{~cm} \times 18 \mathrm{~cm}$, with $1 \mathrm{~cm}^{2}$ unit area for each bin, a total of 3600 bins describes a full rate or contrast map.
} 
rate $R^{\prime}$ over the entire area relative to the total rate $R_{0}$ from the VDC measurement. Both variables approach 1 if the shapes of VDC partial track projected map and scanner's rate map are very similar. However, these two summary variables only partially reflect the features of the rate map, because the rate map $R(x, y)$ is a complex $3 \mathrm{D}$ object.

Fig. 5.17 shows the mean contrasts and total-rate ratios at different beam currents. The data are from run 6615 at $10 \mu \mathrm{A}$, run 6616 at $50 \mu \mathrm{A}$, run 9710 at $145 \mu \mathrm{A}$, run 10044 at $150 \mu \mathrm{A}$ and run 11431 at $165 \mu \mathrm{A}$. The blue line represents the average contrast of these runs, and the red line represents the average total-rate ratio. The uncertainty for each data point is calculated from the rate change when the deadtime is varied by its RMS value. In most scanner runs, the RMS widths of the measured deadtime are less than $1 \mathrm{~ns}$, and the $1 \mathrm{~ns}$ deadtime variations correspond to assigning an uncertainty of about $2.5 \%$ to the measured accidental values.

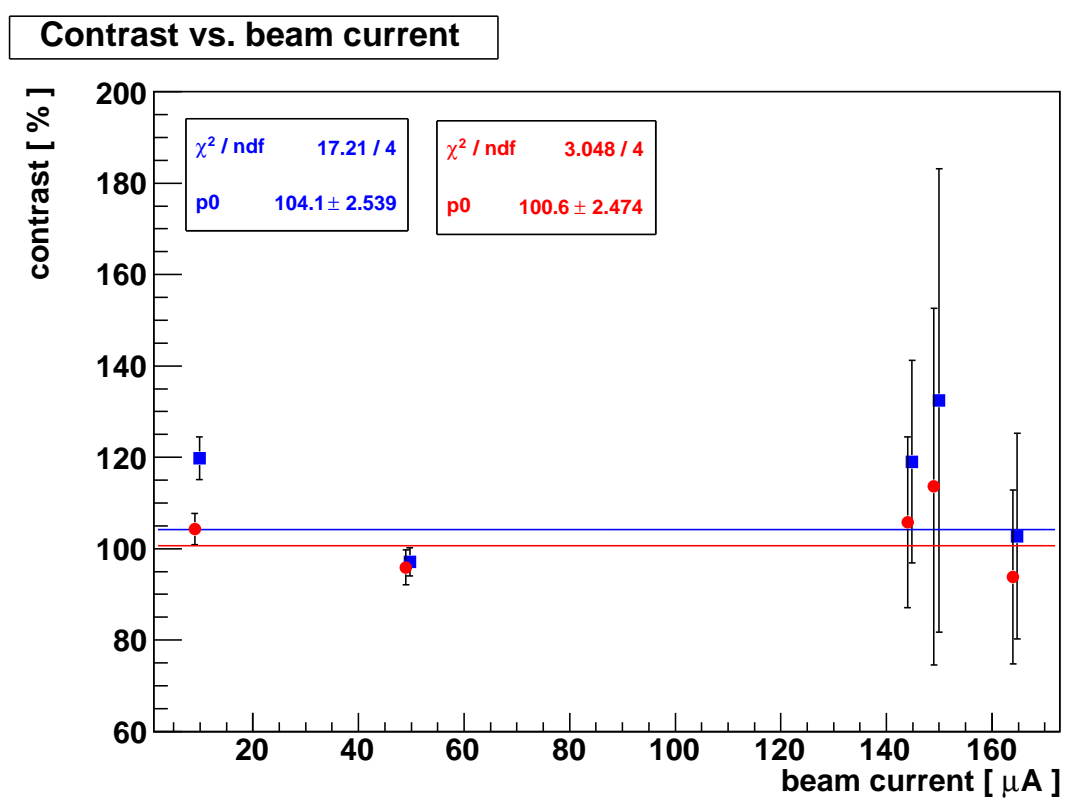

Figure 5.17: The mean contrast (blue marker and line) described by Eqn. 5.14, and the total-rate ratio (red marker and line) described by Eqn. 5.15, of the scanner measured rate distribution, at several different beam currents.

Comparing with the $25 \mathrm{nA}$ VDC run, the scanner's contrast map at $10 \mu \mathrm{A}$ appears to be relatively 


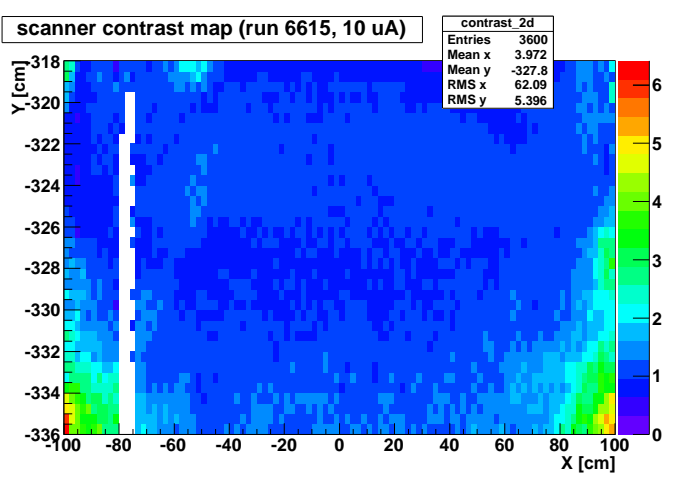

(a) The contrast map

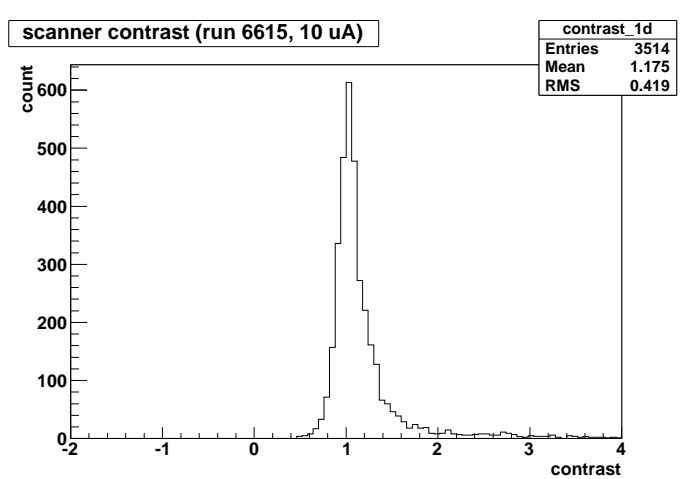

(b) The contrast histogram

Figure 5.18: The scanner's contrast map $C\left(I_{l}, x_{i}, y_{i}\right)$ described by Eqn. 5.13 for run 6615 at $10 \mu \mathrm{A}$ beam current. The color scale in figure (a) and the $x$-axis in figure (b) indicate the contrast values. The white strip in figure (a) arises from a data cut due to a beam trip.

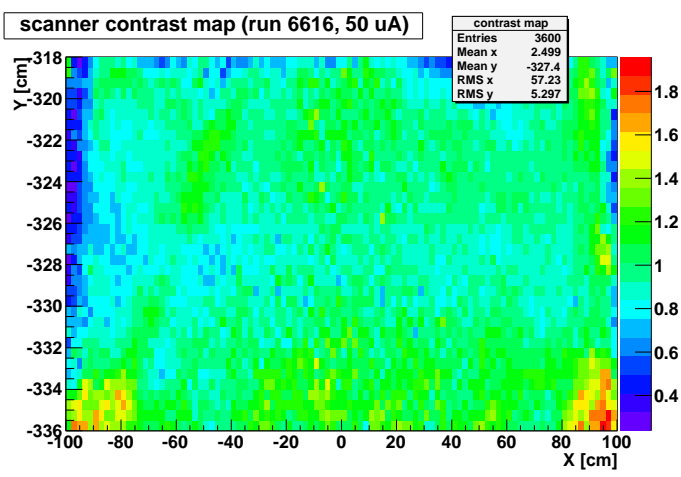

(a) The contrast map

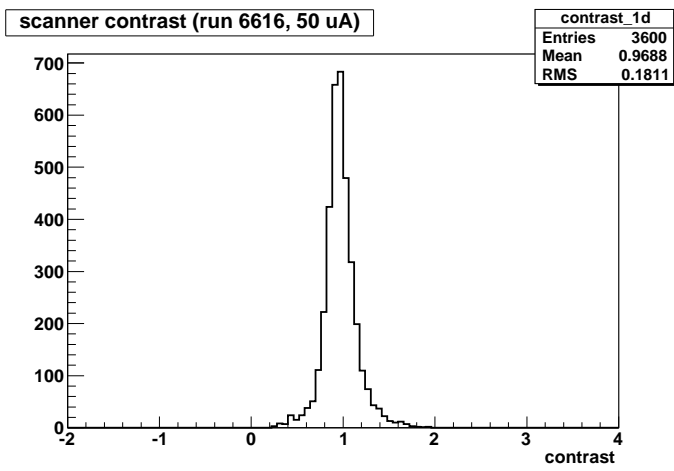

(b) The contrast histogram

Figure 5.19: Scanner's contrast map for run 6616 at $50 \mu \mathrm{A}$ beam current.

uniform in the central region (see Fig. 5.18). The contrast distribution at other currents was also checked and found even to be more uniform. For example, Fig. 5.19 shows the scanner's contrast distribution at $50 \mu \mathrm{A}$ beam current in run 6616. Large non-uniformities are present at the edges of the scanning area, due to low counting rates and thus large statistical errors in these regions. For example, assuming a constant rate of $1 \mathrm{kHz} / \mu \mathrm{A}$ in these regions and a scaler gating rate of $960 \mathrm{~Hz}$ (MPS trigger), the number of counts in the scaler for each trigger at $10 \mu \mathrm{A}$ beam current would be 
$1 \mathrm{kHz} / \mu \mathrm{A} \times 10 \mu \mathrm{A} / 960 \mathrm{~Hz} \simeq 10$. With this low number of counts, even a LSB (least significant bit) error in the scaler would cause a $10 \%$ error on the measured rate. In addition, the VDCs have a relatively low number of hits (hence low statistical precision) in the low rate regions, which also contributes to the apparent non-uniformities. In high rate regions, however, the scanner map is relatively uniform compared to the VDC map.

Fig. 5.20 gives the rate values at three high rate scanner positions (see Fig. 5.11 for the three bin locations), and at different beam currents. The linear response of the scanner at the central bin position demonstrates the feasibility of using the central bin normalization method to compare the shapes of the rate maps at different beam currents.

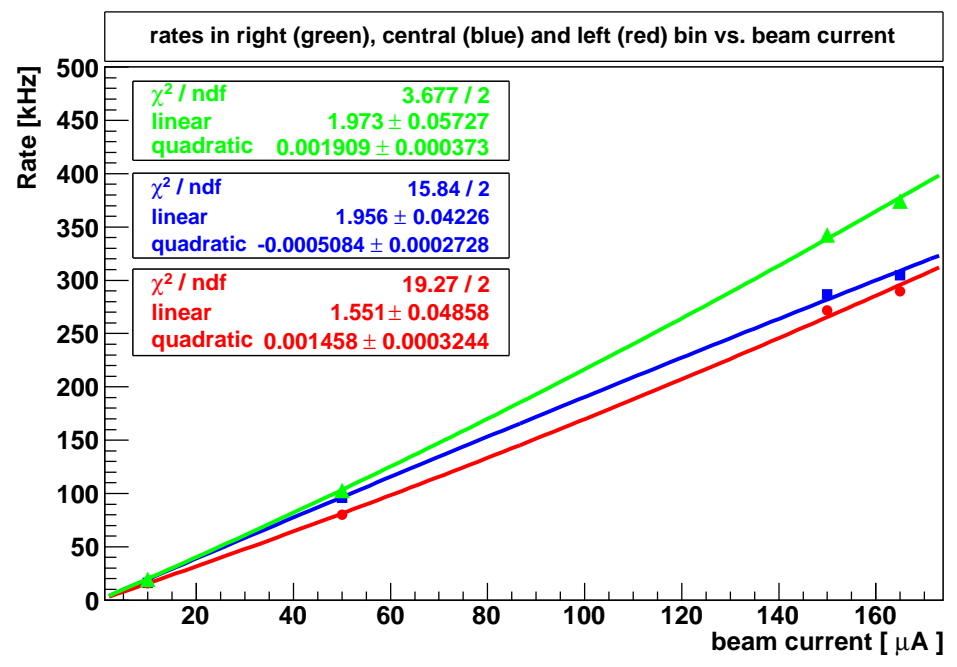

Figure 5.20: The rates at the left bin $(-50 \mathrm{~cm},-322.5 \mathrm{~cm})$ in red, central bin $(0 \mathrm{~cm},-322.5 \mathrm{~cm})$ in blue and right bin $(50 \mathrm{~cm},-322.5 \mathrm{~cm})$ in green versus beam current. The data points are taken from run 6615 at $10 \mu \mathrm{A}$, run 6616 at $50 \mu \mathrm{A}$, the average of run 10044, 10913,10988, 10991 at $150 \mu \mathrm{A}$ and run 11431 at $165 \mu \mathrm{A}$. The error bar on each data point were assigned by varying deadtime by $1 \mathrm{~ns}$, and each set of data was fitted to $f(x)=a x+b x^{2}$. 


\subsubsection{Light-weighted $\left\langle Q^{2}\right\rangle$ Extrapolation}

As discussed in Chapter 4, the light-weighted $\left\langle Q^{2}\right\rangle$ is determined by the tracking system at low beam current (see Eqn. 4.21) in event mode. The light-weighted $Q^{2}$ is a function of hit position $(x, y)$, number of hits $(N)$ and the main detector light yield (\#PE). From low to high beam current, if the relative event rate at every position does not change, i.e. if the geometric shape of the rate map does not change, the light-weighted $\left\langle Q^{2}\right\rangle$ should remain constant. The scanner contrast $\left(C\left(I_{l}, x_{i}, y_{j}\right)\right)$ map can detect a geometric shape change of the rate distribution. Therefore, it is feasible to use $C\left(I_{l}, x_{i}, y_{j}\right)$ to extrapolate the light-weighted $\left\langle Q^{2}\right\rangle$ to different beam currents.

Eqn. 4.21 may be rewritten in a slightly more compact notation as

$$
\left\langle Q^{2}\right\rangle_{0}=\frac{\sum P E_{i} \cdot Q_{i}^{2}}{\sum P E_{i}}
$$

where $P E_{i}$ and $Q_{i}^{2}$ are the number of photo-electrons detected by the main detector PMTs and the 4-momentum transfer squared for event $i$, respectively; the subscript " 0 " is used to represent a low beam current tracking run, at which $Q^{2}$ is determined. In terms of event rate in a bin $k$ of the VDC rate map, Eqn. 5.16 can be written as:

$$
\left\langle Q^{2}\right\rangle_{0}=\frac{\sum N_{k}\langle P E\rangle_{k} \cdot\left\langle Q^{2}\right\rangle_{k}}{\sum N_{k}\langle P E\rangle_{k}}=\frac{\sum R_{k}\langle P E\rangle_{k} \cdot\left\langle Q^{2}\right\rangle_{k}}{\sum R_{k}\langle P E\rangle_{k}}
$$

in which $\langle P E\rangle_{k}$ is the mean number of photo-electrons in bin $k,\left\langle Q^{2}\right\rangle_{k}$ is the mean of $Q_{i}^{2}$ in bin $k$, and $N_{k}$ is the number of hits in bin $k$, which is proportional to the event rate $R_{k}{ }^{3}$ in that bin (noting that an event is indexed by $i$, and a bin is indexed by $k$ ). Eqn. 5.17 gives the light-weighted $\left\langle Q^{2}\right\rangle$ determined from track reconstruction, VDC hits and the main detector response. The extrapolation to beam current $I$ is done by weighting the light yield contribution of events with the scanner contrast, according to:

$$
\left\langle\mathrm{Q}^{2}\right\rangle_{\text {ext }}(I)=\frac{\sum C_{k} R_{k}\langle P E\rangle_{k} \cdot\left\langle Q^{2}\right\rangle_{k}}{\sum C_{k} R_{k}\langle P E\rangle_{k}}
$$

\footnotetext{
${ }^{3}$ It worth noting that the event rate used in this section refers to the rate normalized to beam current.
} 
This extrapolation is based on the assumption that $\left\langle Q^{2}\right\rangle_{k}$ and $\langle P E\rangle_{k}$ do not change from low to high beam current, and that only the relative rate distribution might change with beam current. It also assumes changes in $C_{k}$ observed by the scanner would also be seen by the main detector. Other assumptions could be tested if a source of current-varying $C_{k}$ were found.

Because the extrapolation is performed by using the contrast $C_{k}$, the systematic uncertainty of the extrapolation can be estimated by evaluating the uncertainty of the contrast in each bin,

$$
\Delta\left\langle Q^{2}\right\rangle_{e x t}(\text { syst. })=\frac{\sum\left|\delta C_{k}\right| R_{k}\langle P E\rangle_{k} \cdot\left\langle Q^{2}\right\rangle_{k}}{\sum C_{k} R_{k}\langle P E\rangle_{k}}
$$

$\delta C_{k}$ involves both statistical and systematic contributions. The statistical uncertainty is estimated by

$$
\left.\delta C_{k} \text { (stat. }\right)=\frac{\delta R_{k}^{\prime}(\text { stat } .)}{R_{k}}=\frac{R M S\left(R_{k}^{\prime}\right)}{\sqrt{n} R_{k}},
$$

where $R_{k}^{\prime}$ is the mean of rates measured by the scanner in bin $k$, and $n$ is the number of rate values filled in that bin. Indeed, $\delta R_{k}^{\prime}$ has both statistical and systematic contributions; $\delta C_{k}$ (stat.) is dominated by statistics since the variation of rate with scanner position is generally small. The systematic uncertainty is mainly associated with the accidental rate measurement, which is equivalent to the deadtime determination. For most scanner runs, the deadtime has an RMS width $\left(\sigma_{\tau}\right)$ of less than $1 \mathrm{~ns}$, and therefore the value $\sigma_{\tau}=1 \mathrm{~ns}$ was selected to estimate the systematic uncertainty. Larger deadtime deviations, such as $\sigma_{\tau}=2 \mathrm{~ns}$ or more, could be investigated in further analysis. If we assign $\sigma_{\tau}$ as the uncertainty of the deadtime, the systematic uncertainty of the scanner contrast can be estimated by:

$$
\delta C_{k}(\text { syst. })=\frac{\left|R_{k}^{\prime}\left(\tau \pm \sigma_{\tau}\right)-R_{k}^{\prime}(\tau)\right|}{R_{k}} .
$$

The total uncertainty of $C_{k}$ is obtained by combining $\delta C_{k}$ (stat.) and $\delta C_{k}$ (syst.) in quadrature. Comparing to the systematic uncertainty, the statistical uncertainty is negligible. This estimate provides the systematic uncertainty of the extrapolation due to the scanner detector itself. The uncertainties due to $Q^{2}$ mapping by the VDCs and the photo-electron yield of the main detectors are not included; 
these effects would correspond to an overall scale error in $\left\langle Q^{2}\right\rangle$.

Fig. 5.21 shows the comparison of the determined light-weighted $Q^{2}$ map in octant 5, and the extrapolated light-weighted $Q^{2}$ distribution by the scanner in octant 7. Ideally, the $\left\langle Q^{2}\right\rangle$ extrapolation in the scanner octant should use the reconstructed track and $Q^{2}$ data in the same octant. However, for lack of tracking results in octant 7 due to hardware and geometry issues associated with Region 2 chambers which result in very low track bridging efficiencies in all octants but octant 1 and 5, the $Q^{2}$ data in octant 5 were used here, with an assumption that the $Q^{2}$, number of photo-electron and rate distributions for octant 5 and 7 are the same. The $\left\langle Q^{2}\right\rangle$ extrapolation results at several different beam currents are shown in Fig. 5.22, which indicates that the average of the five extrapolation results agrees with the value of the determined light-weighted $\left\langle Q^{2}\right\rangle$.

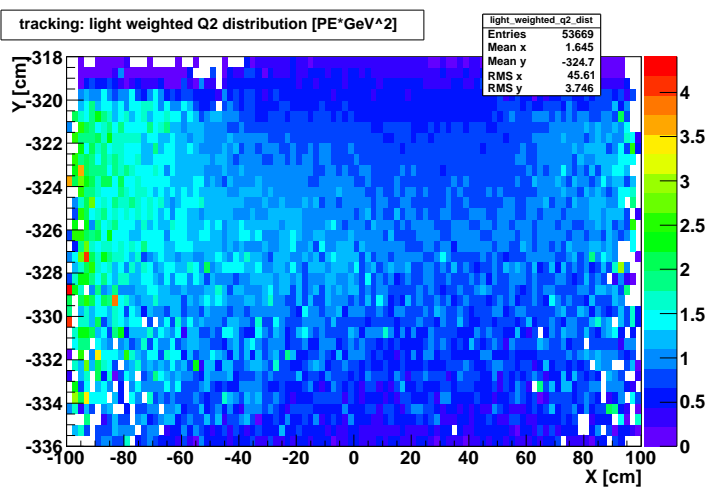

(a)

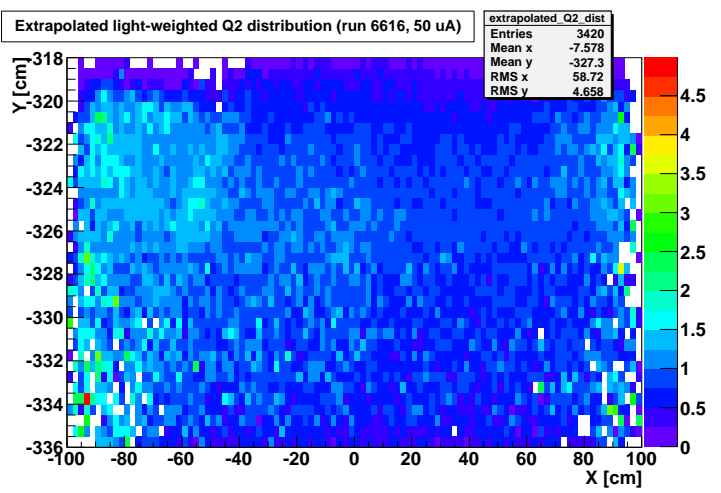

(b)

Figure 5.21: (a) The light-weighted $Q^{2}$ distribution measured in tracking run 8658 at $50 \mathrm{pA}$ in octant 5. (b) The extrapolated light-weighted $Q^{2}$ distribution using scanner data (run 6616) at $50 \mu \mathrm{A}$ in octant 7.

A comparison displayed in Fig. 5.23 shows that the rate and light (number of photo-electron) yield distributions for octant 5 and octant 7 are indeed different due to the differences of detectors, magnetic fields and shielding from octant to octant. Left-right asymmetries of these distributions can also be observed. Table 5.2 summarizes the left-right asymmetries of several different distributions measured by tracking detectors or the scanner at various beam currents. Because the systematic uncertainty of tracking measurements is still under evaluation, the uncertainties of tracking results 


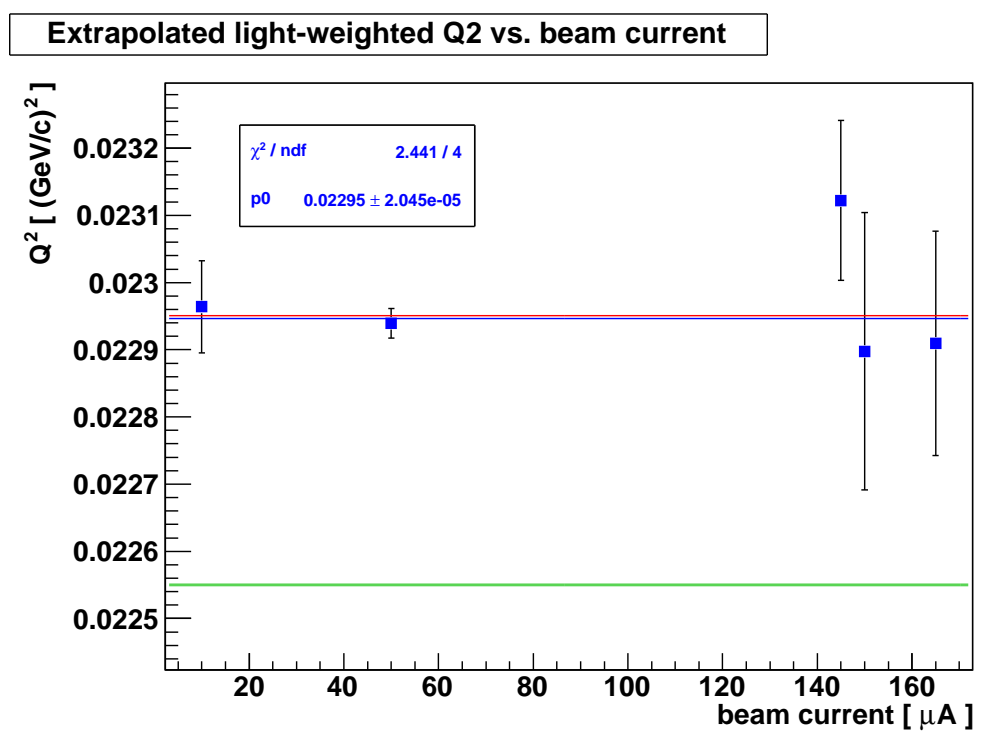

Figure 5.22: The extrapolated light-weighted $\left\langle Q^{2}\right\rangle$ based on the scanner data at five different beam currents. The average of five extrapolation results (blue line at $0.02295 \pm 0.00002(\mathrm{GeV} / \mathrm{c})^{2}$ ) and the determined light-weighted $\left\langle Q^{2}\right\rangle$ (red line at $\left.0.022951(\mathrm{GeV} / \mathrm{c})^{2}\right)$ from the tracking run and the $\left\langle Q^{2}\right\rangle$ before light-weighting (the green line at $\left.0.02255(\mathrm{GeV} / \mathrm{c})^{2}\right)$ are also shown in this plot. The extrapolated light-weighted $\left\langle Q^{2}\right\rangle$ value at $145 \mu \mathrm{A}$ is relatively larger than those from other beam currents because of bad experimental conditions (tune beam, bad halo rate) in that run.

showing in this table are statistical only. For the scanner measurements, the lower current data seem to show smaller uncertainties. To confirm that scanner results in octant 7 could be applied to octant 5, and with what confidence, we would propose that more complete tracking runs be taken so that the true electron rate distributions could be compared. We would still need $C_{k}$ for other octants, to truly extrapolate those octants with current, which would require reworking the scanner support structure for mounting in different octants. Alternatively, a reasonable systematic uncertainty for $C_{k}$ could be derived that would allow application of this technique to other octants. We attempt no such evaluation here. 


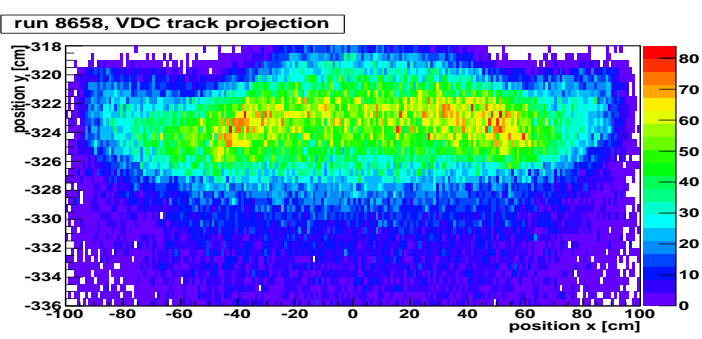

(a) Octant 5: rate distribution

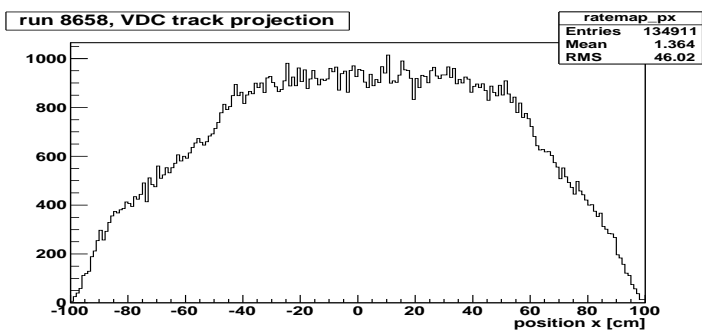

(c) Octant 5: rate distribution projected to $x$

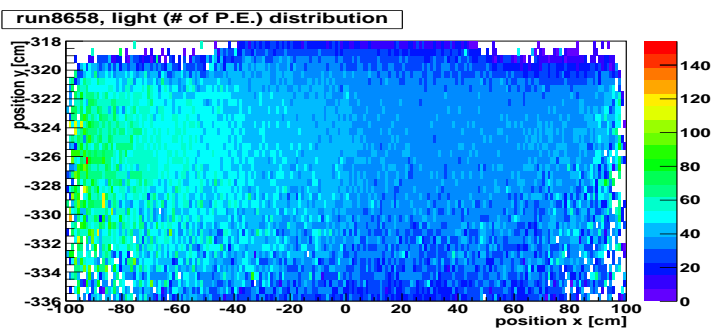

(e) Octant 5: main detector light yield distribution run 8658, light (\# of P.E.) distribution

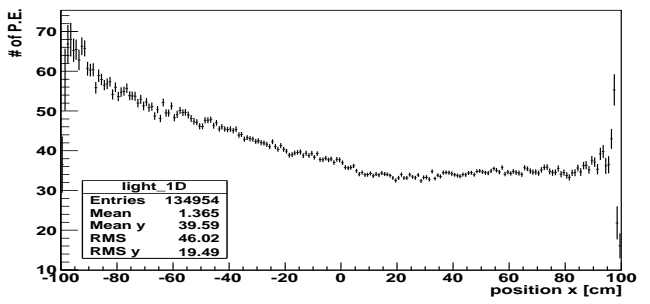

(g) Octant 5: average light yield along quartz bar

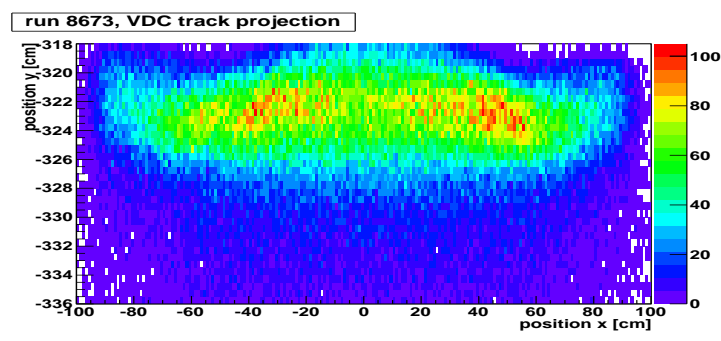

(b) Octant 7: rate distribution

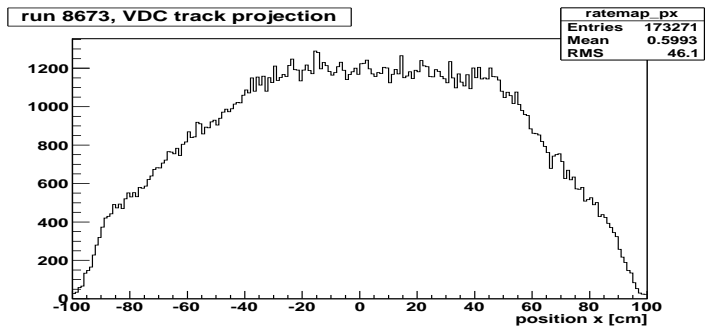

(d) Octant 7: rate distribution projected to $x$

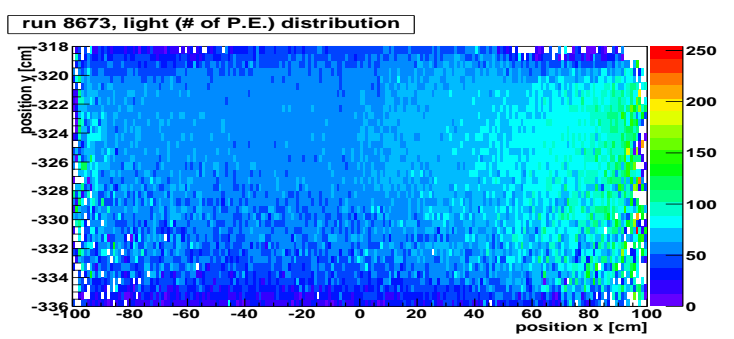

(f) Octant 7: main detector light yield distribution run 8673, light (\# of P.E.) distribution

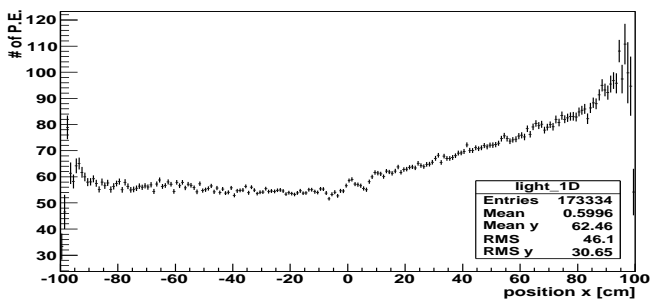

(h) Octant 7: average light yield along quartz bar

Figure 5.23: Comparison of rate and light (\# of P.E.) distribution in Octant 5 (run 8658) and 7 (run 8673 ) at $50 \mathrm{pA}$ beam current. Plots are generated by projecting VDC tracks (with main detector light yield cut) to the scanner plane, and the coordinates for octant 5 plots are converted to octant 7 . All coordinates are global coordinates. The color scales indicate the number of hits in the scanner plane and main detector light yields, which were obtained by using the single photo-electron calibration values given in Ref. [124]. 


\begin{tabular}{|c|c|c|c|c|c|}
\hline Run & Octant & $\begin{array}{c}\text { Beam } \\
\text { Current }\end{array}$ & $\begin{array}{l}\text { Distribution } \\
\text { Type }\end{array}$ & $\begin{array}{l}\text { Left-Right } \\
\text { Asymmetry }\end{array}$ & $\begin{array}{l}\text { Figure } \\
\text { Reference }\end{array}$ \\
\hline \multirow[t]{4}{*}{8658} & 5 & $50 \mathrm{pA}$ & $\begin{array}{l}\text { VDC partial track } \\
\text { projection map }\end{array}$ & $-0.026 \pm 0.003$ & $\begin{array}{l}\text { Fig. } 5.23(\mathrm{a}),(\mathrm{c}) \\
\text { and Fig. } 4.22(\mathrm{a})\end{array}$ \\
\hline & & & $\begin{array}{l}\text { ADC pulse height } \\
\text { distribution map }\end{array}$ & $0.099 \pm 0.001$ & \\
\hline & & & $\begin{array}{l}\text { light (\# of PE) } \\
\text { distribution map }\end{array}$ & $0.155 \pm 0.002$ & $\begin{array}{l}\text { Fig. } 5.23(\mathrm{e}),(\mathrm{g}) \\
\text { and Fig. } 4.22(\mathrm{a})\end{array}$ \\
\hline & & & $Q^{2}$ distribution map & $0.015 \pm 0.009$ & Fig. 4.22 (c) \\
\hline \multirow[t]{3}{*}{8673} & 7 & $50 \mathrm{pA}$ & $\begin{array}{l}\text { VDC partial track } \\
\text { projection map }\end{array}$ & $-0.015 \pm 0.003$ & Fig. 5.23 (b), (d) \\
\hline & & & $\begin{array}{l}\text { ADC pulse height } \\
\text { distribution map }\end{array}$ & $-0.049 \pm 0.001$ & \\
\hline & & & $\begin{array}{l}\text { light (\# of PE) } \\
\text { distribution map }\end{array}$ & $-0.131 \pm 0.002$ & Fig. 5.23 (f), (h) \\
\hline \multirow[t]{3}{*}{8672} & 7 & $25 \mathrm{nA}$ & $\begin{array}{l}\text { VDC partial track } \\
\text { projection map }\end{array}$ & $-0.020 \pm 0.003$ & Fig. 5.15 \\
\hline & & & $\begin{array}{l}\text { ADC pulse height } \\
\text { distribution map }\end{array}$ & $-0.049 \pm 0.001$ & \\
\hline & & & $\begin{array}{l}\text { light (\# of PE) } \\
\text { distribution map }\end{array}$ & $-0.129 \pm 0.002$ & \\
\hline 6615 & 7 & $10 \mu \mathrm{A}$ & $\begin{array}{l}\text { normalized scanner } \\
\text { rate map }\end{array}$ & $-0.030 \pm 0.001$ & Fig. B.1 \\
\hline 6616 & 7 & $50 \mu \mathrm{A}$ & $\begin{array}{l}\text { normalized scanner } \\
\text { rate map }\end{array}$ & $-0.034 \pm 0.003$ & Fig. B.2 \\
\hline 9710 & 7 & $145 \mu \mathrm{A}$ & $\begin{array}{l}\text { normalized scanner } \\
\text { rate map }\end{array}$ & $-0.029 \pm 0.010$ & Fig. B.3 \\
\hline 10044 & 7 & $150 \mu \mathrm{A}$ & $\begin{array}{l}\text { normalized scanner } \\
\text { rate map }\end{array}$ & $-0.065 \pm 0.010$ & $\begin{array}{l}\text { Fig. B.4 and } \\
\text { Fig. } 5.10 \text { (b) }\end{array}$ \\
\hline 11431 & 7 & $165 \mu \mathrm{A}$ & $\begin{array}{l}\text { normalized scanner } \\
\text { rate map }\end{array}$ & $-0.051 \pm 0.010$ & Fig. B.5 \\
\hline
\end{tabular}

Table 5.2: The left-right asymmetries of several distributions. For a distribution of a variable $\alpha$ in the focal plane, we define its left-right asymmetry as $A_{L R}=\left(L_{\alpha}-R_{\alpha}\right) /\left(L_{\alpha}+R_{\alpha}\right)$, where $L_{\alpha}$ is the sum of $\alpha$ on the left half-bar (for $x<0$ ), and $R_{\alpha}$ is the sum of $\alpha$ on the right half-bar (for $x>0$ ). $A_{L R}$ reflects the asymmetric feature of the distribution image. 


\subsection{Other Applications}

In addition to obtaining the focal plane flux profile and performing $\left\langle Q^{2}\right\rangle$ extrapolation, the scanner has several other applications; monitoring spectrometer optics is one of them. Because the scanner detector can quickly obtain the rate distribution in the focal plane, it can serve as a minimally invasive monitor. In order to do this, it is necessary to know the scanner's sensitivities to the variables to be monitored, as discussed below.

\section{Scanner Sensitivities}

The rate distribution image acquired by the scanner, $R\left(x_{i}, y_{j}\right)$, is sensitive to many effects from beam properties and experimental settings and conditions. The measured rate distributions are mainly characterized by the following parameters:

- $X_{C}$ is the $x$-location of the rate distribution centroid $\left(X_{C}=\frac{\sum_{i, j} x_{i} R\left(x_{i}, y_{j}\right)}{\sum_{i, j} R\left(x_{i}, y_{j}\right)}\right)$,

- $Y_{C}$ is the $y$-location of the rate distribution centroid $\left(Y_{C}=\frac{\sum_{i, j} y_{j} R\left(x_{i}, y_{j}\right)}{\sum_{i, j} R\left(x_{i}, y_{j}\right)}\right)$,

- $X_{p}$ is the $x$-location of the rate distribution peak,

- $Y_{p}$ is the $y$-location of the rate distribution peak,

- $R_{\max }$ is the maximum rate.

These parameters may be grouped to form a vector $P=\left(X_{C}, Y_{C}, X_{p}, Y_{p}, \ldots\right)$, which may be affected by experimental condition changes. A major factor is the QTOR DC current $I_{\text {qtor }}$. Other variables, such as beam parameters (beam position $x, y$, beam angles $\theta_{x}, \theta_{y}$ ), target location in $z$-direction $\left(Z_{t g t}\right)$, also influence the rate distribution. These experimental variables may be used to construct another vector $V$. For $P$ with $m$ elements and $V$ with $n$ elements, a $m \times n$ sensitivity matrix $S$ is defined to describe the relation between $P$ and $V$, as:

$$
P=S V
$$


or more explicitly:

$$
\left(\begin{array}{c}
X_{C} \\
Y_{C} \\
X_{p} \\
Y_{p} \\
R_{\max }
\end{array}\right)=\left(\begin{array}{ll}
S_{11} & S_{12} \\
S_{21} & S_{22} \\
S_{31} & S_{32} \\
S_{41} & S_{42} \\
S_{51} & S_{52}
\end{array}\right)\left(\begin{array}{c}
I_{q t o r} \\
Z_{\text {tgt }}
\end{array}\right),
$$

where for simplicity, we take five distribution parameters and two experimental variables only. To first order and neglecting correlations between variables, the sensitivity matrix element $S_{i j}$ is given by

$$
S_{i j}=\frac{\partial P_{i}}{\partial V_{j}}, \quad(i=1 \ldots m, j=1 \ldots n)
$$

For the purpose of monitoring spectrometer optics, we focus on the sensitivity to the QTOR magnetic field (QTOR current) variations. Several scanner runs were taken to measure this sensitivity parameter.

\section{$\mathrm{LH}_{2}$ Target Data}

The rate distribution in the focal plane is very sensitive to the variation of the spectrometer magnetic field. Small variations in the QTOR current $I_{q t o r}$ will move the rate image position radially. Therefore, the scanner can be used to monitor the spectrometer optics by performing fast vertical strip scans along the $y$-direction at $x=0$, where the rate variation is most pronounced.

A typical central strip scan takes less than 30 seconds, and a profile of rate distribution along the $y$-direction can be quickly obtained. If we describe the obtained profile with the parameters $Y_{p}$ and $R_{\max }$, the corresponding sensitivity elements are $\frac{\partial Y_{p}}{\partial I_{q t o r}}$ and $\frac{\partial R_{\max }}{\partial I_{q t o r}}$, describing how $Y_{p}$ and $R_{\max }$ vary with $I_{q t o r}$.

Table 5.3 shows the peak position in $y$ of the scanner profile and the maximum rate with respect to the QTOR current in run 10951, from which the sensitivities can be deduced. The peak position sensitivity at nominal QTOR current $\left(I_{q t o r}=8921 \mathrm{~A}\right)$ is $\frac{\partial Y_{p}}{\partial I_{q t o r}} \simeq 1.78 \mathrm{~cm} /\left(1 \% \times I_{q t o r}\right)$, i.e. a $1 \%$ 
magnetic field variation ${ }^{4}$ will change the location of scanned rate peak by about $1.78 \mathrm{~cm}$. The maximum raw rate (no deadtime correction and calibration, normalized to beam current) sensitivity at $8921 \mathrm{~A}$ QTOR current is $\frac{\partial \hat{R}_{\max }}{\partial I_{q t o r}} \simeq 0.34 \mathrm{kHz} / \mu \mathrm{A} /\left(1 \% \times I_{q t o r}\right)$.

\begin{tabular}{ccc}
\hline \hline QTOR current (A) & Peak position in $y(\mathrm{~cm})$ & Max. raw rate $(\mathrm{kHz} / \mu \mathrm{A})$ \\
\hline 8750 & 332.0 & 5.2 \\
8921 & 335.8 & 5.6 \\
9000 & 337.0 & 5.3 \\
\hline \hline
\end{tabular}

Table 5.3: The Peak position and maximum rate at three different QTOR currents in the QTOR scan run 11951 (75 $\mu \mathrm{A}$ beam current on $\mathrm{LH}_{2}$ target). During this run, the scanner took a central strip scan at $13.9 \mathrm{~cm}$ downstream of the main detector.

During the experiment, the nominal QTOR current is measured and monitored by a DCCT (DC current transformer), which has a high accuracy of 0.1 A. Although the DCCT is sensitive to QTOR current drifts, the variations in rate distribution due to $I_{\text {qtor }}$ changes need to be confirmed by scanner measurements, especially during parity runs.

\section{Al Target Data}

Similar studies were performed for the scanner detector upstream of the main detector, measuring the rate profile of a $1 \%$ radiation length upstream aluminum solid target [80], which has the same $z$-location as the $\mathrm{LH}_{2}$ target cell entrance window. The rate maps measured in runs 11994 and 11995 with two different $I_{q t o r}$ settings are compared in Fig. 5.24. The rate map projections in $y$ shown in Fig. 5.25 indicates that the peak of the profile shifts $2.1 \mathrm{~cm}$, for a $1.5 \%$ increase in magnetic field, corresponding to a sensitivity of $\frac{\partial Y_{p}}{\partial I_{q t o r}}=1.4 \mathrm{~cm} / 1 \%$.

Changes in the rate map due to variation of the target $z$-position were observed. Fig. 5.26 shows a rate map for run 11695. In this run, the downstream aluminum solid target [80], which has the same $z$-location of the $\mathrm{LH}_{2}$ target cell exit window, was used. Comparing the rate maps of this run with the upstream aluminum solid target runs, we observe a $\sim 5.3 \mathrm{~cm}$ shift of the rate map profile

\footnotetext{
${ }^{4} \mathrm{QTOR}$ is a DC iron-free magnet, therefore $\Delta B / B_{0}=\Delta I / I_{0} .1 \%$ current change causes a $1 \%$ magnetic field variation.
} 


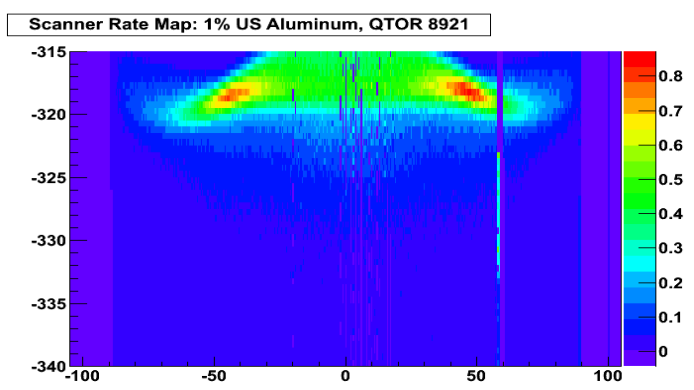

(a) rate map for run 11994

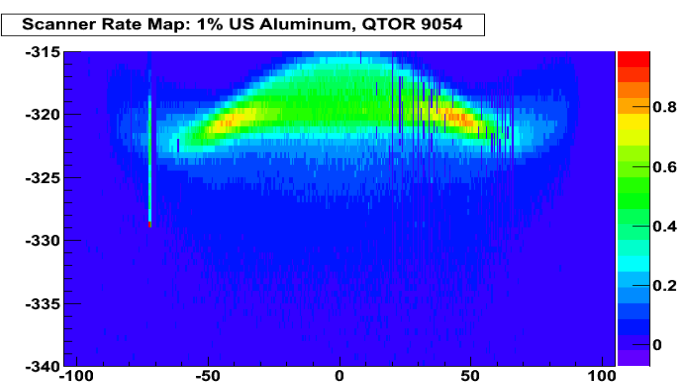

(b) rate map for run 11995

Figure 5.24: The rate maps for run 11994 and run 11995 with $65 \mu \mathrm{A}$ beam current on the $1 \%$ upstream (US) aluminum solid target with QTOR currents of 8921 A and 9054 A, respectively [125]. The $x$ - and $y$-axes are the Q-weak global $x$ - and $y$-coordinates in units of $\mathrm{cm}$. The color scales indicate the rate in arbitrary units.

peak in $y$.

\section{Scanner PMT Spectra and Pre-radiator Studies}

Fig. 5.27 shows the scanner PMT pulse height spectra, which were taken during full-range rate measurements. In contrast to the TRIUMF beam tests and cosmic tests (see Fig. 3.10 and Fig. 3.13 for reference), which were triggered by the coincidence of two trigger paddles, these spectra were obtained with a scanner self-coincidence trigger. Therefore, no pedestals appear in either spectra. Once other triggers, such as a trigger scintillator trigger or a main detector trigger, were selected, large pedestal peaks would appear due to the small scanner active area relative to that for the trigger detector.

At JLab, the scanner PMTs are operated at a lower gain than that at TRIUMF so as to be able to work at very high rates. Therefore, the single photo-electron peak cannot be resolved from the QDC spectra. Estimates of the charge corresponding to a single photo-electron were made based on oscilloscope observations. According to measured spectra and pedestals, the scanner light yields were estimated by

$$
\# \text { of } \mathrm{PE}=\left(\text { channel }_{\text {mean }}-\text { channel }_{\text {ped. }}\right) /\left(\text { channel }_{1 \mathrm{PE}}-\text { channel }_{\mathrm{ped}}\right) .
$$




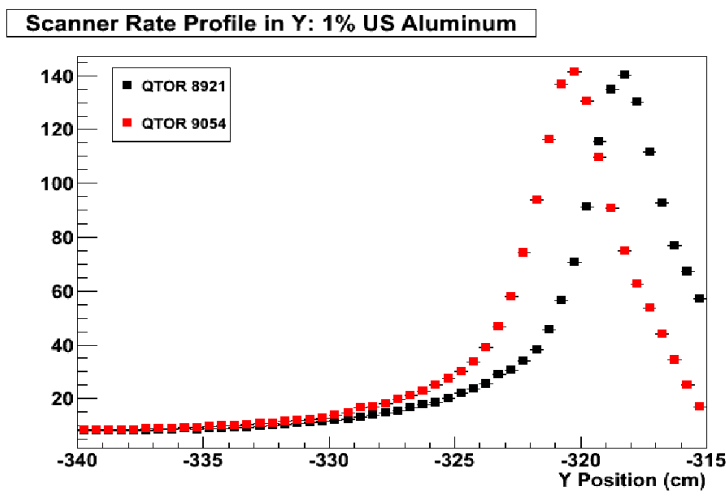

Figure 5.25: The comparison of the profile projections in global $y$ for run 11994 and 11995 at two different QTOR currents of 8921 A and 9054 A, respectively [125].

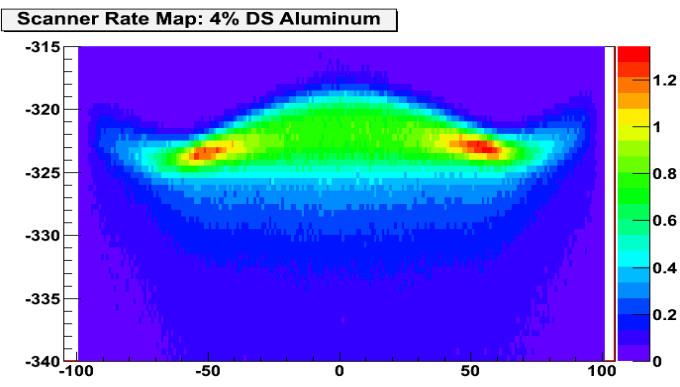

(a)

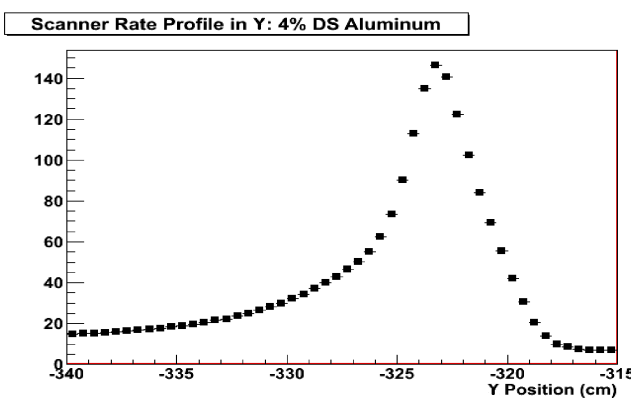

(b)

Figure 5.26: (a) The rate map for run 11695 with $26 \mu \mathrm{A}$ beam current on the $4 \%$ downstream (DS) Aluminum solid target at the nominal QTOR current (8921 A). The $x$ - and $y$-axes are the Q-weak global $x$ - and $y$-coordinates in units of $\mathrm{cm}$. The color scale indicates the rate in arbitrary units. (b) The rate map projection onto global $y$ [125].

The channel numbers for the pedestals were confirmed from the data triggered by the trigger scintillator. To reduce backgrounds from the detector itself, both of the two PMT thresholds were set to $\sim 3$ photo-electrons in hardware for all measurements. This implies $\sim 70 \%$ scanner efficiencies according to simulations (see Fig. 3.18) and experimental data (as discussed in Section 5.2.1).

As noted earlier, the scanner detector can perform measurements both upstream and downstream of the main detector. In the downstream location, besides a slight increase in the detector's photoelectron yield, the signal-to-background ratio $(\mathrm{S} / \mathrm{B})$ was also improved by the pre-radiator of the 


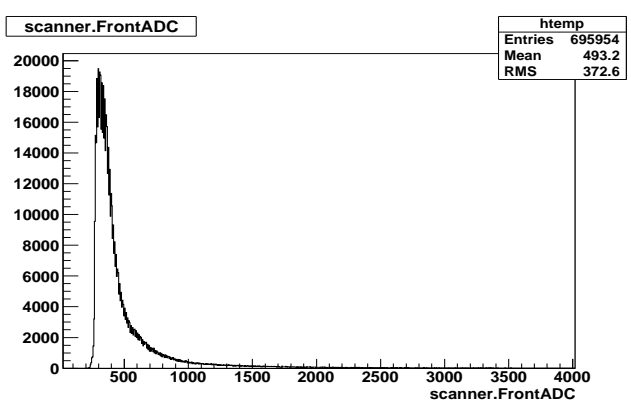

(a) upstream, front PMT

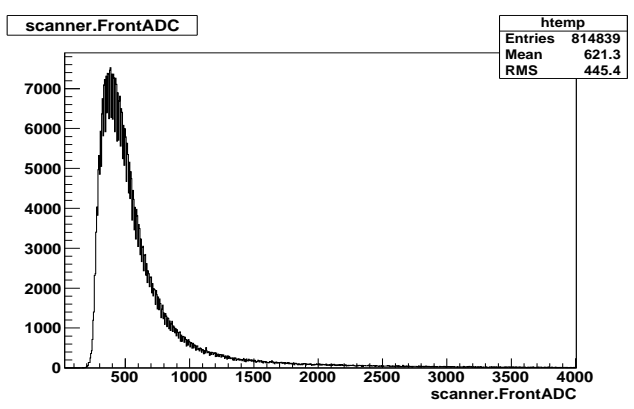

(c) downstream, front PMT

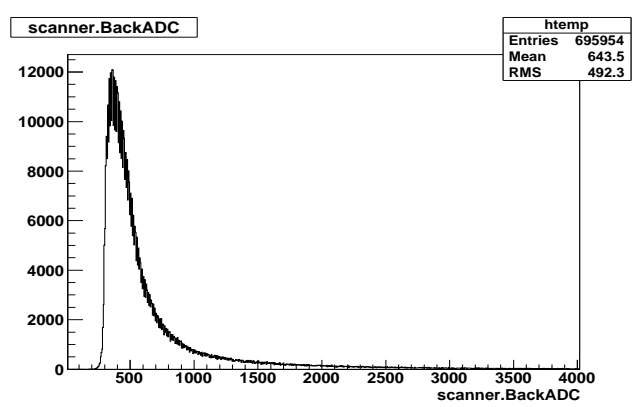

(b) upstream, back PMT

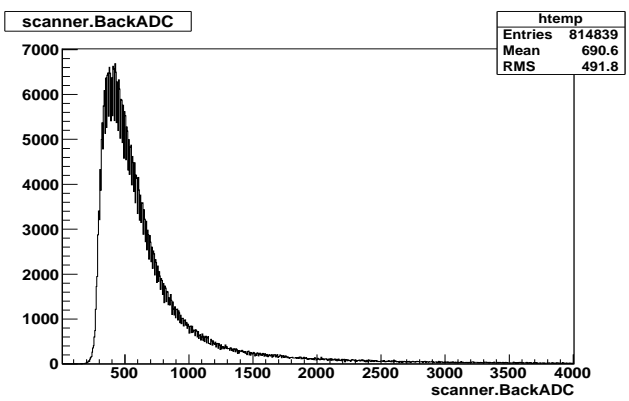

(d) downstream, back PMT

Figure 5.27: Scanner charge ADC spectra taken upstream (run 6616, $50 \mu \mathrm{A}$ beam current, $\mathrm{LH}_{2}$ target) and downstream (run 10949, $75 \mu \mathrm{A}$ beam current, $\mathrm{LH}_{2}$ target) of the main detector. The $x$-axis indicates the ADC channel number, with each channel corresponding to a charge of $0.1 \mathrm{pC}$.

main detector. The measured scanner rate was increased about a factor of 2.5 at the downstream location. Although the scanner detector and the main detector have different responses to the preradiator due to their different $z$-locations and active areas, the scanner detector could be a useful diagnostic device to study the effects of the main detector pre-radiator.

\subsection{Summary and Outlook}

In the first Q-weak run cycle, a very limited data set was available for the scanner. Therefore, the current work has focused on outlining the scanner analysis methodology. It is planned to make further technical improvements on the scanner, and to make more tracking and scanner measurements 
during the second Q-weak run period, to complete a full data set for superior $Q^{2}$ determination and to develop the extrapolation techniques first investigated here. 


\section{Chapter 6}

\section{Parity Data Analysis}

The goal of the parity data analysis is to obtain the physics asymmetry from the raw main detector asymmetry, so as to extract the proton's weak charge $Q_{W}^{p}$. There are two main branches in the Q-weak analysis leading to the $Q_{W}^{p}$ determination, as depicted in Fig. 6.1, including the physics asymmetry obtained by the parity analysis and the $\left\langle Q^{2}\right\rangle$ determined by the tracking analysis. A method of reconstructing and extrapolating $\left\langle Q^{2}\right\rangle$ to high beam current has been introduced in the previous chapters. Procedures for extracting the physics asymmetry from the raw asymmetry via experimental corrections will be the main focus of this chapter. As the Run I data analysis is not fully completed at this stage, only preliminary results for raw asymmetries will be reported. The status of the systematic uncertainty will also be reviewed.

\subsection{General Analysis Procedures}

The Q-weak parity data are collected during production runs with current mode main detectors and electronics. During data taking, the beam helicity state is reversed with a frequency of $960 \mathrm{~Hz}$, while the signals from the main detectors and related beam line components are integrated in each helicity state (MPS window) and recorded in CODA format. Typically, each parity run contains an hour's worth of data, segmented in about 10 data files (or runlets). The raw data are stored on the 


\section{Parity}

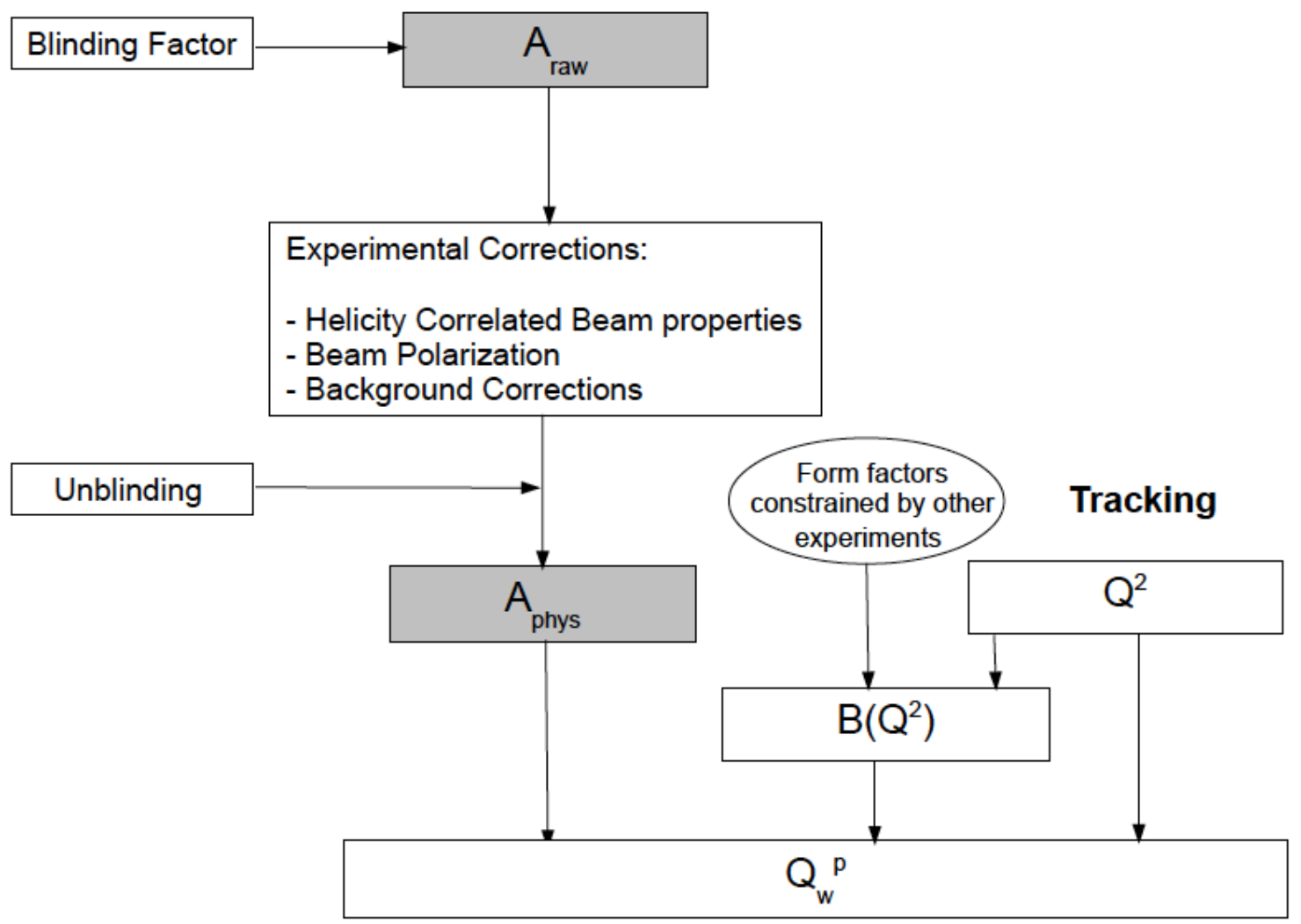

Figure 6.1: Flow chart of the Q-weak analysis procedures. There are two main branches: the parity branch for physics asymmetry determination and the tracking branch for $Q^{2}$ determination. In parity analysis, experimental corrections need to be applied to the raw asymmetry to extract physics asymmetry, and blinding analysis is adopted in this procedure. The blinding factors used in Q-weak Run I were randomly taken from $-60 \mathrm{ppb}$ to $+60 \mathrm{ppb}$.

hard drives of the DAQ computers, and later transferred onto tape silo storage for off-line analysis.

When processing the data in one run, the parity analyzer first decodes the CODA events into physical quantities, such as detector PMT yields (the signal amplitude of PMT output current, determined by event rate, number of photo-electron yield and PMT gain) and beam parameters. Raw asymmetries are calculated based on the yields of Čerenkov bars within each pseudo-random quartet helicity pattern (“+ - -+" or "- + +-"). The data are processed in several "passes", and each pass has different calibrations and data cuts. During the first pass, initial beam current cuts and device 
failure cuts are applied, to remove data taken during beam trips and excursions. The beam charge asymmetry and the helicity-correlated differences of other beam parameters are also calculated. All these quartet-based quantities may then be saved as ROOT [126] histograms and trees for further analysis. Run-based means and uncertainties of detector yields, asymmetries, and beam parameters are stored in a MySQL [127] database to facilitate the later query, retrieval and analysis.

In order to extract the physics asymmetry, various corrections need to be applied. Detector and $\mathrm{BCM}$ calibrations are needed to arrive at main detector yields. The false asymmetries due to helicity-correlated beam properties must be removed by using linear regression techniques. The effects caused by beam polarization and background dilutions also need to be taken into account. Details of these corrections will be discussed in Section 6.3. To avoid human bias when analyzing data, a blinded analysis is used. A blinding factor is added to the raw asymmetry and will not be revealed until all analyses are finalized. The unknown blinding factor is therefore present in all the data shown here.

\subsection{Raw Asymmetry}

\subsubsection{Initial Data Processing}

To extract the main detector normalized yields for asymmetry calculations, several corrections need to be applied, as described below.

Pedestal Subtraction The main detector consists of eight octants, and the yield ${ }^{1}$ of each detector is recorded by two PMT channels. The pedestals of those channels, i.e. the DC offsets of the electronics chains, including dark current due to thermal noise and cosmic rays, must be subtracted from the raw signals so that real detector yields can be obtained. The pedestals may change periodically due to variations in temperature, room backgrounds, etc. To account for these effects, brief pedestal

\footnotetext{
${ }^{1}$ For the main detectors, each PMT yield is referred to as the total number of photo-electrons collected by the PMT photocathode in unit time, which is then amplified by the PMT and converted into a DC voltage signal via a transimpedance amplifier. The yield of one detector is defined as the sum of the two PMTs' yields on the Cerenkov bar.
} 
runs are taken once or twice per day with beam off during production data taking. The average pedestal values are subtracted from the yield of each PMT channel. The subtraction is done with the most recently updated pedestal values.

PMT Gain Match The light yield seen by one PMT decreases with distance from the PMT along the quartz bar. The sum of the two PMTs' light yields exhibits reduced position dependence. In order to sum the two PMT signals for one detector to obtain the detector's yield, or to sum the total 16 PMT signals to obtain the yield for all detectors, the gains of those PMT channels should be well matched to each other. The gain is adjusted by adjusting each PMT's high voltage. Weighting factors are used in the analysis software to further equalize the PMTs' yields. These factors are determined based on weighting each PMT's yield by the average yield of 16 PMTs taken from a good quality production run.

Beam Current Normalization In order to reduce the dependence on beam intensity fluctuations, the main detector yields are normalized to beam current (or beam charge) after pedestal subtraction. The normalization is done in each MPS according to a particular BCM's readout. BCMs are calibrated periodically in dedicated runs.

\subsubsection{Data Selection}

The main detector yields are processed in several analysis passes. In each pass, it is necessary to check data quality and apply improved calibrations and cuts. During preliminary data quality selection, beam trip, beam excursion, and hardware failure cuts are imposed. Events that have been taken with unstable beam conditions, with electronic glitches, DAQ failures, and/or detector problems are rejected. In order to avoid systematic bias, cuts are optimized to stretch over some time period. For example, beam trips must give the $\mathrm{LH}_{2}$ target enough time to recover. In subsequent passes through the data analysis, more stringent cuts and improved analysis will be applied, which require the events outside of acceptable beam parameter limits to be removed from the data set. For data taken with large charge asymmetries due to charge feedback malfunctions, substantial fractions 
of a typical 1 hour run or more might be eliminated. The data used in this thesis are from the first pass analysis.

\subsubsection{Raw Asymmetry Calculation}

Raw Asymmetry for a Single Detector The normalized detector yield is proportional to the differential cross section in e-p elastic scattering. Therefore, according to the definition of the asymmetry $A_{P V}=\left(\sigma_{R}-\sigma_{L}\right) /\left(\sigma_{R}+\sigma_{L}\right)$, the measured asymmetry may be expressed in terms of the detector yield as:

$$
A_{m d i}=\frac{Y_{i}^{+}-Y_{i}^{-}}{Y_{i}^{+}+Y_{i}^{-}}
$$

where $A_{m d i}(i=1-8)$ is the asymmetry of the $\mathrm{i}$-th main detector, $Y_{i}^{+}$and $Y_{i}^{-}$are the detector yields (sum of two PMTs' yields, weighted by their weighting factors) for the "+" and "-" helicity states of a quartet, respectively. As mentioned earlier, the beam helicity is reversed in a pseudo random quartet pattern (“+--+" or " -++- "); the detector asymmetry in one quartet is calculated by using Eqn. 6.1, with the $Y_{i}^{+}$and $Y_{i}^{-}$corresponding to the yield sum of two "+" and two "-" helicity states in the quartet, respectively.

Raw Asymmetry for All Detectors The overall experimental asymmetry is obtained from $A_{m d a l l}$, the combination of all eight main detectors' asymmetry. $A_{m d a l l}$ is calculated in the same way as that for the $A_{m d i}$ mentioned above, but by summing the yields from the total 16 PMT channels together. The combined asymmetry is calculated for each quartet, and with more data being accumulated, a histogram of the quartet-by-quartet asymmetry forms a Gaussian distribution. The mean of the running average asymmetry and its statistical width can then be expressed by:

$$
\left\langle A_{\text {mdall }}^{\text {run }}\right\rangle=\frac{1}{N_{\text {qrt }}} \sum_{i}^{N_{\text {qrt }}} A_{\text {mdall }, i},
$$


and

$$
\sigma\left(A_{\text {mdall }}^{\text {run }}\right)=\left[\frac{1}{N_{q r t}} \sum_{i}^{N_{q r t}}\left(A_{\text {mdall }, i}-\left\langle A_{\text {mdall }, i}^{\text {run }}\right\rangle\right)^{2}\right]^{\frac{1}{2}},
$$

respectively, where $N_{q r t}$ is the total number of good quartets in the run and $A_{m d a l l, i}$ is the asymmetry for the i-th quartet.

In addition to the yield-based combination, the $A_{\text {mdall }}$ for a runlet or a run can also be calculated with another option - the asymmetry-based combination. With this method, the $A_{\text {mdall }}^{\text {run }}$ is obtained by averaging the $A_{m d i}$ for single detectors separately, using an appropriate statistical weight $\left(1 / \sigma\left(A_{m d i}\right)^{2}\right)$ from each detector. If the measurements of the detectors are independent, the asymmetries obtained using these two methods should agree. Fig. 6.2 shows an example of both the $A_{m d i}$ and $A_{m d a l l}$ distributions (in ppm) obtained from a production run 12023. The detector asymmetry plots for MD1 - MD8 are arranged in clockwise direction following the octant order defined in the Q-weak coordinate system. The central plot shows the combination asymmetry for the whole main detector array. Each detector measures the quartet-to-quartet asymmetry distribution with $\sim 650 \mathrm{ppm}$ width, providing the combination asymmetry width of $\sim 240 \mathrm{ppm}$ in this $165 \mu \mathrm{A}$ run.

Main detector asymmetries for a series of runs are combined using a weighted mean based on the statistical uncertainty of each run. Explicitly, the averaged asymmetry over runs is calculated by using

$$
A_{\text {total }}=\frac{\sum_{\text {run }} A_{\text {run }} / \delta\left(A_{\text {run }}\right)^{2}}{\sum_{\text {run }} 1 / \delta\left(A_{\text {run }}\right)^{2}},
$$

where the $\delta\left(A_{\text {run }}\right)$ represents run-based statistical uncertainty of $A_{\text {run }}$. The width and the statistical uncertainty for $A_{\text {total }}$ are given by

$$
\sigma\left(A_{\text {total }}\right)=\left[\sum_{\text {run }} \frac{1}{\sigma\left(A_{\text {run }}\right)^{2}}\right]^{-\frac{1}{2}}
$$

and

$$
\delta A_{\text {total }}=\frac{\sigma\left(A_{\text {total }}\right)}{\sqrt{N_{q r t}}}
$$



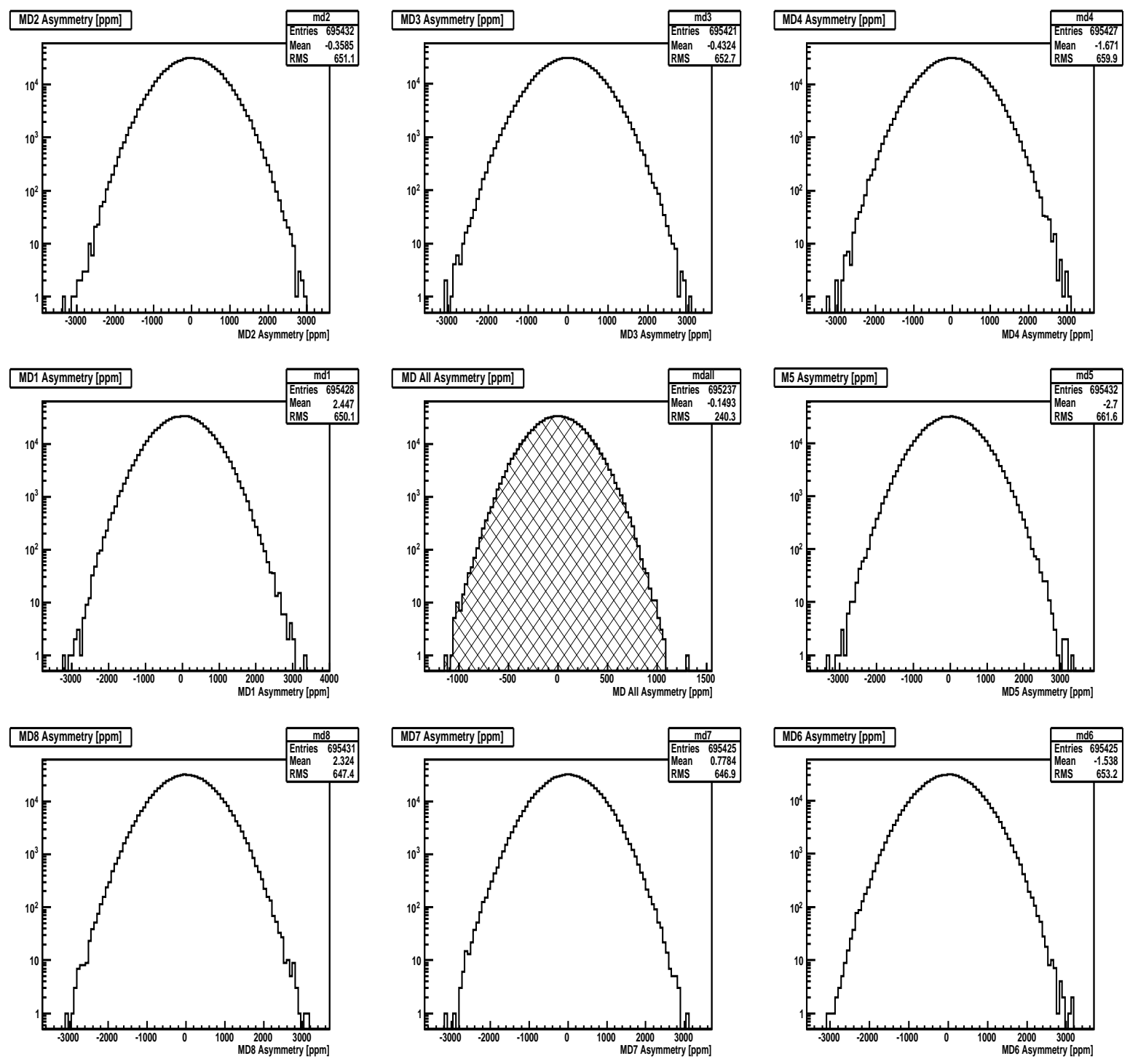

Figure 6.2: The asymmetry measured by each main detector and the combination of the whole main detector array in run 12023, with $165 \mu \mathrm{A}$ beam current, $4 \mathrm{~mm} \times 4 \mathrm{~mm}$ raster size, and $\mathrm{LH}_{2}$ target.

respectively, where the $N_{q r t}$ is the total number of good quartet patterns accumulated in the runs. 


\subsubsection{Data Quality in Run I}

Before further discussion of correction procedures to extract the physics asymmetry, it is necessary to check the quality of Run I data. Two main aspects are addressed: the statistical properties, and systematic effects.

\section{Statistical Property Check}

As mentioned above, a histogram of the quartet-by-quartet asymmetries should form a Gaussian distribution. Fig. 6.3 shows an example asymmetry distribution with a width of $240 \mathrm{ppm}$, acquired in a run at a beam current of $165 \mu \mathrm{A}$. The total number of good quartets ("Entries") and the measured asymmetry width indicate that a $288 \mathrm{ppb}$ statistical uncertainty was achieved within the $\sim 50$ minute measurement.

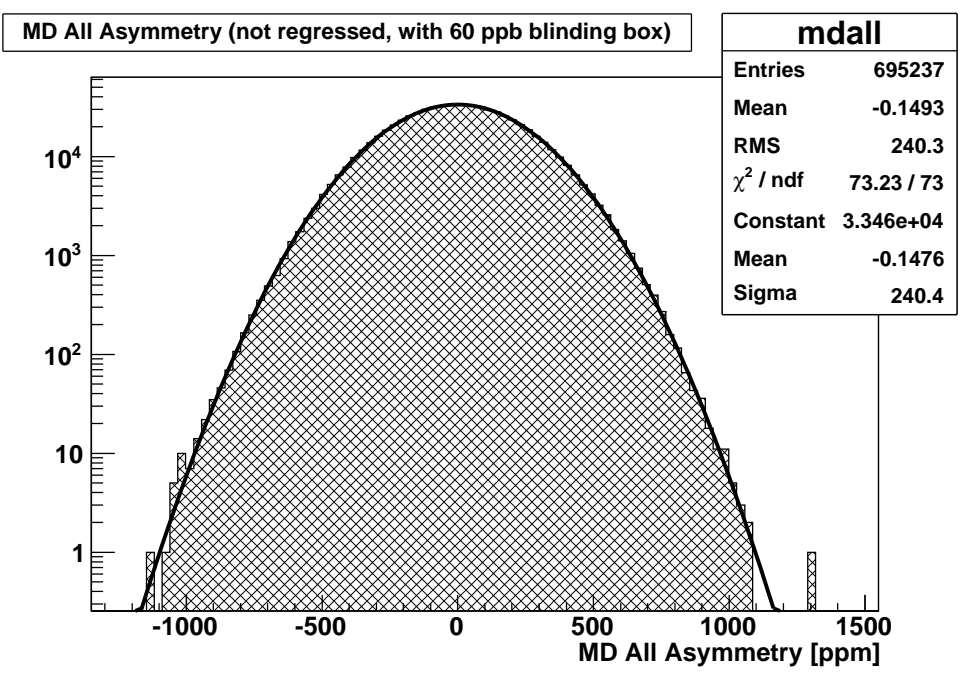

Figure 6.3: The raw asymmetry measured by main detectors in run 12023 at a beam current of $165 \mu \mathrm{A}$. The asymmetry distribution in this plot was fitted to a Gaussian.

Ideally, the asymmetry width would be as close as possible to that implied by counting statistics $\sigma_{\text {count }}$. In real measurements, the asymmetry width will be broadened by many noise factors, such as the detector resolution $\sigma_{d e t}$, target boiling noise $\sigma_{t g t}$, beam current normalization $\sigma_{B C M}$, electronics 
noise $\sigma_{\text {elec }}$, etc., which if uncorrelated with one another contribute to the total width in quadrature:

$$
\sigma(A)=\sqrt{\sigma_{\text {count }}^{2}+\sigma_{\text {det }}^{2}+\sigma_{\text {tgt }}^{2}+\sigma_{B C M}^{2}+\sigma_{\text {elec }}^{2}+\ldots} .
$$

According to simulations, at a beam current of $165 \mu \mathrm{A}$, the total rate detected by the main detectors is $R=5.87 \mathrm{GHz}$, which gives an asymmetry distribution width $\sigma_{\text {count }}$ due to pure counting statistics:

$$
\sigma_{\text {count }}=\frac{1}{\sqrt{R t}}=\frac{1}{\sqrt{5.87 \mathrm{GHz} \times \frac{1}{960 \mathrm{~Hz}} \times 4 \times 93 \%}}=210 \mathrm{ppm}
$$

where $t$ is the counting time for one quartet with a 93\% helicity reversal live-time [128].

The main detector resolution, which reflects the relative RMS noise width of the detector signals and is defined as " $\frac{\sigma}{\text { mean }}$ " of the photo-electron yield distribution obtained from event mode calibration runs, of $\sim 45 \% \sigma_{\text {count }}$ will contribute $\sim 10 \%$ excess noise above counting statistics [85], i.e.

$$
\sqrt{\sigma_{\text {count }}^{2}+\sigma_{\text {det }}^{2}}=\sqrt{\sigma_{\text {count }}^{2}\left(1+45 \%{ }^{2}\right)}=\sigma_{\text {count }}(1+10 \%)
$$

When taking the $\sigma_{d e t}$ term into account, the asymmetry width is expected to be increased to 231 ppm. Other contributions arising from BCM measurements for current normalization $\left(\sigma_{B C M} \sim 50\right.$ ppm) and target boiling noise $\left(\sigma_{\text {tgt }} \sim 50 \mathrm{ppm}\right)$, lead to a slight increase in the width to $242 \mathrm{ppm}$. Electronic noise is about 3 orders of magnitude below counting statistics, and is therefore negligible. The observed width of $240 \mathrm{ppm}$ in this run is therefore close to expectation.

As the statistical uncertainty projection plot shows in Fig. 6.4, if Q-weak continues data taking with a typical asymmetry width of $235 \mathrm{ppm}$ and $87 \%$ beam polarization during Run II, a 2.3\% measurement of the asymmetry can be reached with a reasonable operation efficiency of $50 \%$, and the final goal of $2.1 \%$ can be achieved with an efficiency of $70 \%$ within the scheduled beam time. Taking account of the time given to other studies, such as periodic tracking measurements, beam polarization measurements with the Møller polarimeter, accelerator beam studies, and other downtime, the typical operation efficiency is about $50 \%$. 


\section{$\Delta$ A/A Projections (assumes 235 ppm MD, 87\% pol)}

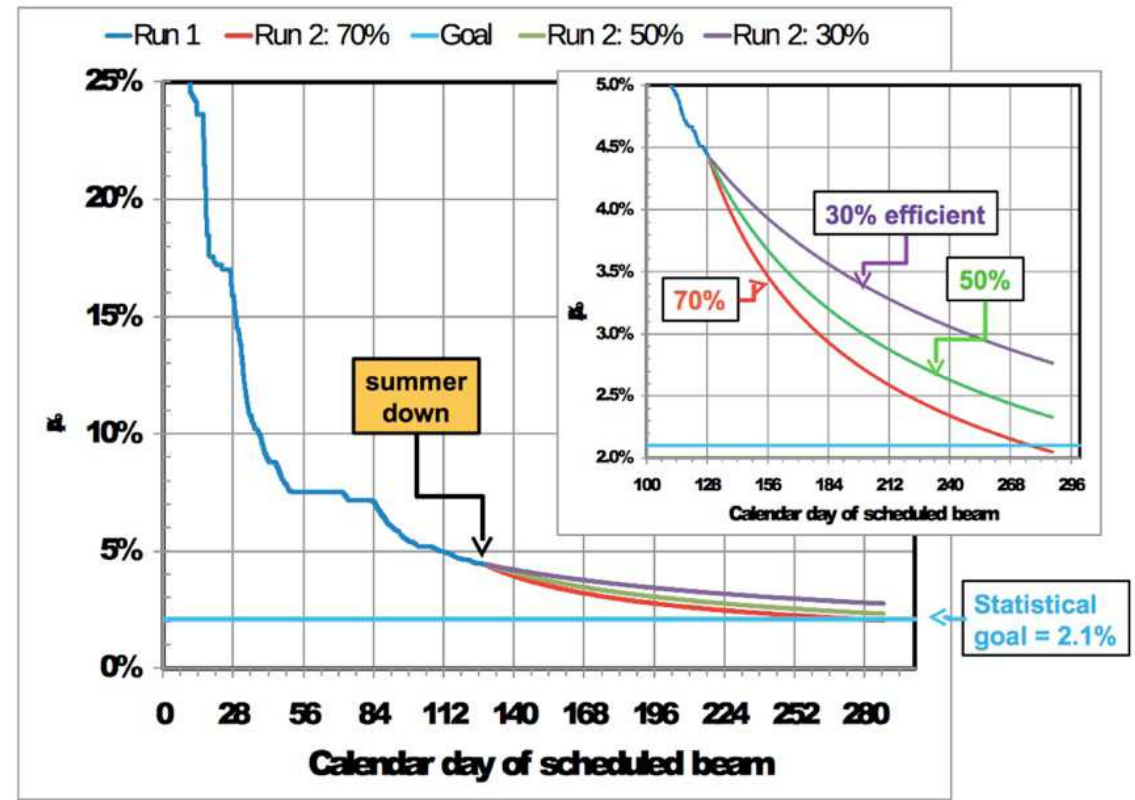

Figure 6.4: Evaluation of the expected statistical uncertainty $(\Delta \mathrm{A} / \mathrm{A})$ versus scheduled beam time (in calendar days) under the assumption of $30 \%, 50 \%$ and $70 \%$ operation efficiencies [128].

\section{Systematic Checks}

During data taking, the helicity state is reversed every $\sim 1 \mathrm{~ms}$ at the injector. As mentioned in Section 2.2.2, slow helicity reversals are performed periodically by changing the IHWP and Wien filter states to study possible systematic effects on a relatively longer timescale. The IHWP state is reversed every eight hours being placed in either the IN or OUT state, and the Wien filter is changed to Left (L) or Right (R) about once per week. The combination of IHWP and Wien settings thus leads to four different sign-flip configurations: IN-L, OUT-L, IN-R and OUT-R.

Runs are grouped into "slugs", corresponding to different IHWP and Wien settings. One 8 hourlong data slug is collected with a given IHWP state, and the sign of the asymmetry is alternately reversed slug-by-slug by inserting or retracting the IHWP, with Wien filter settings unchanged. This reverses the sign of measured "IN" and "OUT" asymmetries, and the "IN + OUT" summed 
asymmetry should therefore be consistent with zero.

Fig. 6.5 shows an example of the raw asymmetry versus slug number under two source configurations. The sign of the average asymmetry reverses for the different IHWP states. Considered as two separate datasets, the two IHWP settings also show good statistical agreement in the measured asymmetries. The slower Wien-flip allows further systematic tests on longer timescales. During commissioning and Run I, a total of 136 slugs were collected, which have been grouped in six Wien periods. The result of the average asymmetry in each Wien configuration will be discussed in Section 6.5.

Asymmety vs. Slug (Q-weak Run I, Preliminary, Blinded)

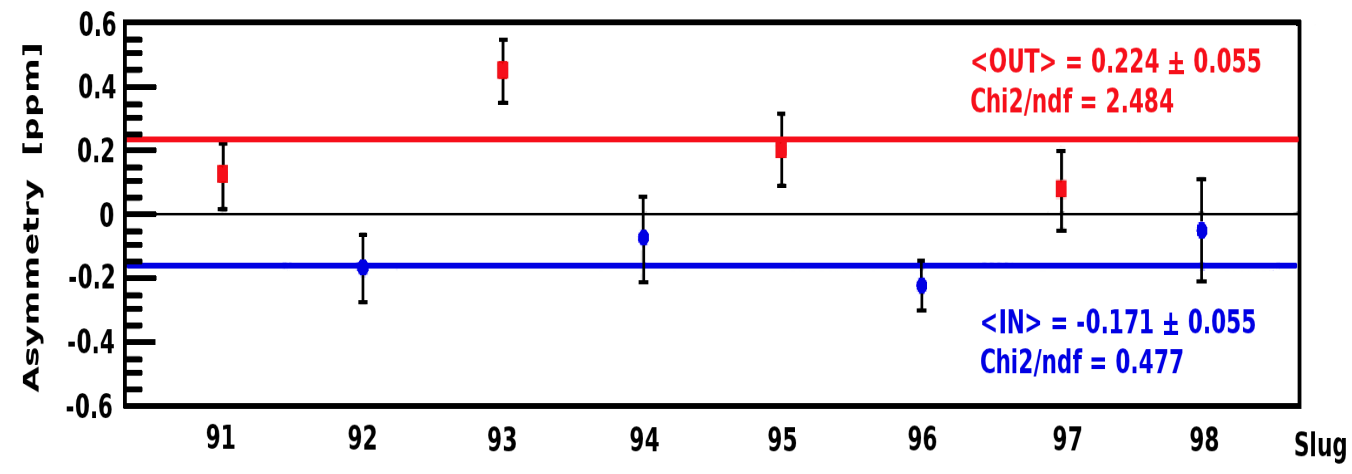

Figure 6.5: A "slug plot" for production runs with the $\mathrm{LH}_{2}$ target [129]. The data in these slugs were taken under two different IHWP configurations, and the average asymmetries for "IN" and "OUT" slugs appear in opposite sign as they should.

\subsection{Applying Corrections}

Corrections need to be applied to the raw asymmetry in order to obtain the physics asymmetry. In this section, basic correction methods are introduced. The preliminary analysis results and the status of the Q-weak Run I data will then be discussed in Section 6.5 (Physics corrections are discussed in Section 6.4). 


\subsubsection{Linear Regression}

Helicity-correlated beam property differences $\delta B$, such as beam energy $\left(E^{+}-E^{-}\right)$, charge $\left(Q^{+}-Q^{-}\right)$, positions $\left(x^{+}-x^{-}, y^{+}-y^{-}\right)$and angles $\left(\theta_{x}^{+}-\theta_{x}^{-}, \theta_{y}^{+}-\theta_{y}^{-}\right)$, may lead to significant false asymmetries. To first order, these systematic effects (at a few ppb level) can be removed through linear regression. In linear regression, the raw asymmetry is regressed against the beam parameters by subtracting a linear combination of all beam monitor asymmetries:

$$
A^{r e g}=A^{r a w}-\sum_{m} C_{m} \delta B_{m}
$$

where $\delta B_{m}$ is the helicity-correlated beam property difference or asymmetry for the $m$-th beam parameter. The factors $C_{m}=\partial A / \partial B_{n}$ are the sensitivities (regression slopes), which are obtained from fitting a straight line to $A^{\text {raw }}$ versus $\delta B_{m}$ correlation plots.

If the correlations between the beam parameters are non-negligible, higher order effects have to be taken into account. The sensitivities, $C_{m}$, involve the correlations of the detector asymmetry with respect to different beam parameters. In order to find the best fit value of $C_{m}$, one minimizes the $\chi^{2}$ function:

$$
\chi^{2}=\left\langle\frac{1}{\left(\delta A^{\text {raw }}\right)^{2}}\left(A^{\text {raw }}-\sum_{m} C_{m} \delta B_{m}\right)^{2}\right\rangle
$$

with respect to $C_{m}$ (assuming $\delta A^{\text {raw }}=$ constant), leading to a solution:

$$
\operatorname{Cov}\left(A^{r a w}, \delta B_{n}\right)=\sum_{m} C_{m} \operatorname{Cov}\left(\delta B_{m}, \delta B_{n}\right)
$$

in which the covariance of two variables $\alpha$ and $\beta$ is represented by

$$
\operatorname{Cov}(\alpha, \beta)=\langle(\alpha-\langle\alpha\rangle)(\beta-\langle\beta\rangle)\rangle=\langle\alpha \beta\rangle-\langle\alpha\rangle\langle\beta\rangle
$$

The angle brackets " \langle\rangle " in the above expressions represent the mean value of a random variable. $\delta A^{\text {raw }}$ in Eqn. 6.11 is the uncertainty of the asymmetry measurement for each quartet. The minimum 
$\chi^{2}$ is found when Eqn. 6.12 is satisfied. Eqn. 6.12 can be written explicitly in a matrix form as:

$$
\vec{Y}=X \cdot \vec{C}
$$

where

$$
X=\left(\begin{array}{cccc}
\operatorname{Cov}(\delta E, \delta E) & \operatorname{Cov}(\delta E, \delta Q) & \cdots & \operatorname{Cov}\left(\delta E, \delta y^{\prime}\right) \\
\operatorname{Cov}(\delta Q, \delta E) & \operatorname{Cov}(\delta Q, \delta Q) & \cdots & \operatorname{Cov}\left(\delta Q, \delta y^{\prime}\right) \\
\vdots & \vdots & \ddots & \vdots \\
\operatorname{Cov}\left(\delta y^{\prime}, \delta E\right) & \operatorname{Cov}\left(\delta y^{\prime}, \delta Q\right) & \cdots & \operatorname{Cov}\left(\delta y^{\prime}, \delta y^{\prime}\right)
\end{array}\right)
$$

is the covariance matrix of the beam parameters,

$$
\vec{Y}=\left(\begin{array}{c}
\operatorname{Cov}\left(A^{r a w}, \delta E\right) \\
\operatorname{Cov}\left(A^{r a w}, \delta Q\right) \\
\vdots \\
\operatorname{Cov}\left(A^{r a w}, \delta y^{\prime}\right)
\end{array}\right) \quad \text { and } \quad \vec{C}=\left(\begin{array}{c}
C_{1} \\
C_{2} \\
\vdots \\
C_{6}
\end{array}\right)
$$

are the covariance vector and the coefficient vector, respectively. The coefficients $C_{n}$ are then obtained by inverting the covariance matrix and multiplying by the covariance vector, i.e.

$$
\vec{C}=X^{-1} \vec{Y}
$$

The covariances are experimentally determined by performing multidimensional fits of $A^{\text {raw }}$ against the beam parameter differences, as well as single beam parameter differences against each other. Because the coefficients can change over time, they are determined for every run. The status of the linear regression analysis for the Run I data will be discussed in Section 6.5.1.

\subsubsection{Backgrounds Correction}

The experimental asymmetry after regression still involves contributions from backgrounds such as pions, elastic events from aluminum target windows, and soft gammas. Neglecting radiative 
corrections, the experimental asymmetry is given by the detector yield difference over detector yield sum as [54]:

$$
A^{e x p}=P_{b} \frac{A_{e p} Y_{e p}+A_{b k g} Y_{b k g}}{Y_{e p}+Y_{b k g}}
$$

where $P_{b}$ is the beam polarization, determined from the Møller and Compton measurements, the $Y$ 's are the beam-current-weighted detector yields, any $A$ without the superscript "exp" is a physics asymmetry, the subscript " $e p$ " indicates the elastic ep scattering channel and the subscript "bkg" indicates a background channel. By generalizing to an arbitrary number of background reactions, this can be expressed as:

$$
A_{e p}=\frac{A^{e x p}}{P_{b}}+\sum_{j}\left(\frac{A^{e x p}}{P_{b}}-A_{b k g, j}\right) \frac{Y_{b k g, j}}{Y_{e p}} .
$$

From Eqn. 6.19, background processes can affect the measurement by diluting the elastic signal and by exhibiting a non-zero asymmetry. Therefore, to determine the physics asymmetry $A_{e p}$, a correction for each background channel $j$ must be made, which requires that the fractional yield $Y_{b k g, j} / Y_{e p}$ and the difference of the asymmetries $\left(A_{\text {exp }} / P_{b}-A_{b k g, j}\right)$ be known from independent measurements.

Table 6.1 lists the main background sources and estimated contributions, prior to Q-weak running, according to simulations. The largest contributions arise from the aluminum windows of the target cell. Detailed background determination methods have been summarized in Ref. [54]. During Run I, the background dilution factor and asymmetry of the aluminum windows, as well as the contributions from inelastic events, were investigated through both simulations and dedicated measurements (see Section 6.5.3).

\subsubsection{Beam Polarization Correction}

The beam polarization $P_{b}$ in Eqns. 6.18 and 6.19 appears as a normalization factor for the asymmetry $A^{\text {exp }}$. The uncertainty on the $P_{b}$ measurement directly propagates to the asymmetry. Therefore, to reach the proposed experimental accuracy, $P_{b}$ needs to be determined to an uncertainty better than 


\begin{tabular}{ccc}
\hline \hline Background Source & Type & Asymmetry-weighted fractional yield contribution \\
\hline Aluminum target windows & elastic & $11.1 \%$ \\
& quasi-elastic & $-3.3 \%$ \\
\hline Target & inelastic & $-0.5 \%$ \\
\hline Beamline \& & photon & $0.4 \%$ \\
collimators & $e^{+} e^{-}$ & $0.1 \%$ \\
& neutron & $0.4 \%$ \\
\hline \hline
\end{tabular}

Table 6.1: Summary of background source and estimated contributions [54]. The asymmetryweighted fractional yield is defined as $A_{b k g} f$, where $f$ represents the dilution factor for backgrounds, defined as $f=Y_{b k g} /\left(Y_{e p}+Y_{b k g}\right)$.

$1 \%$.

Residual transverse polarization components in the nominally longitudinally polarized beam could cause a false asymmetry if the detectors were not symmetric about the beam axis. The transversely polarized electrons can contribute as a parity conserving Mott asymmetry, arising due to two photon exchange [130]. The correction $\alpha_{T}$ to the parity-violating asymmetry is then:

$$
\alpha_{T}=\beta A_{T}\left(\frac{P_{b}^{T}}{P_{b}}\right)
$$

where $A_{T}$ is the measured asymmetry for purely transverse beam polarization, $P_{b}^{T}$ and $P_{b}$ are the transverse and total beam polarizations, and $\beta$ is the degree of detector geometrical mismatch, which can be known from the octant to octant asymmetry variation in main detectors. In order to determine $A_{T}$, dedicated measurements with transversely polarized beam are needed. Preliminary results for $P_{b}$ and $A_{T}$ measurements will be provided in Sections 6.5.2 and 6.5.4. 


\subsubsection{Hadronic Structure Correction}

The Q-weak experiment aims to determine $Q_{W}^{p}$ from the measured parity-violating physics asymmetry $A_{P V}$, which contains contributions from nucleon structure form factors (see reference [54]):

$$
A_{P V}=A_{Q_{W}^{p}}+A_{\text {vector }}+A_{\text {axial }} .
$$

$A_{Q_{W}^{p}}$ represents the asymmetry contributed by $Q_{W}^{p}$ only at the $Q^{2} \rightarrow 0$ limit. $A_{\text {vector }}$ involves the vector electromagnetic and weak hadronic form factors; it reduces to the $Q^{4} B\left(Q^{2}\right)$ term in Eqn. 1.49 for small $Q^{2}$. $A_{\text {axial }}$ involves the e- $N$ axial form factor $G_{A}^{e}$. Estimates for the hadronic structure contributions to Q-weak are listed in Table 6.2 [54]. These values were determined from the results of other parity-violating electron scattering experiments at higher $Q^{2}$ that are more sensitive to nucleon structure, as discussed in Section 1.3.4.

\begin{tabular}{c|ccc}
\hline \hline & Correction [ppb] & Uncertainty [ppb] & Contribution to $Q_{W}^{p}$ uncertainty \\
\hline$A_{\text {vector }}$ & -101 & 2.83 & $1.5 \%$ \\
$A_{\text {axial }}$ & -12 & 2.16 & $1.2 \%$ \\
\hline Total & -113 & 3.56 & $1.9 \%$ \\
\hline \hline
\end{tabular}

Table 6.2: Summary of estimated hadronic structure corrections at Q-weak kinematics [54].

\subsection{Physics Result Extraction}

For each run, the physics asymmetry is obtained by correcting the raw asymmetry for beam parameters, backgrounds and beam polarization, neglecting radiative corrections. The asymmetry over $N$ runs is obtained by weighting the asymmetry of each run with the corresponding statistical uncertainty (see Eqns. 6.4 - 6.6 for reference, where the weighted mean and statistical uncertainty are calculated). The overall parity-violating asymmetry is given by the weighted mean of all runs. The systematic uncertainty due to beam properties is taken into account in the linear regression 
procedure. The remaining systematic uncertainty is calculated through error propagation from the uncertainties in background measurements and beam polarization measurements.

The physics asymmetry, $A_{Q_{W}^{p}}$, can be deduced by using Eqn. 6.21 to remove the hadronic contributions. The proton's weak charge then would be determined from

$$
Q_{W}^{p}=-\frac{4 \pi \alpha \sqrt{2} A_{Q_{W}^{p}}}{G_{F} Q^{2}},
$$

at the very small $Q^{2}$ determined via Q-weak track reconstruction (the $Q^{2}$ used in this formula indeed represents the "light-weighted $\left\langle Q^{2}\right\rangle$ " determined by QTR), as discussed in Chapter 4.

Alternatively, from the previous discussion in Section 1.3.4, a global fit of the PVES asymmetry to the $Q^{2} \rightarrow 0$ limit would allow an extraction of $Q_{W}^{p}$ from the world data, according to Eqn. 1.50. The Q-weak experiment will provide a new data point of $\overline{A_{P V}}\left(Q^{2}\right)$ to the global fit for $Q_{W}^{p}$ extraction.

The weak mixing angle is then extracted by:

$$
\sin ^{2} \theta_{W}=\frac{1}{4}\left(1-Q_{W}^{p}\right)+\text { radiative corrections }
$$

In fact, to determine the physics asymmetry, hence $Q_{W}^{p}$ and $\sin ^{2} \theta_{W}$, radiative corrections need to be applied to the measured asymmetry. The radiative corrections include the electroweak radiative corrections which have been discussed in Section 1.3.5. Electromagnetic (EM) radiative corrections which account for the electron's energy loss at the scattering vertex due to photon radiation must also be taken into account. These radiative corrections may affect $Q^{2}$ and the asymmetry, and are mainly accounted for in the $\left\langle Q^{2}\right\rangle$-determination and background determination as discussed in Section 4.4.5. Ultimately, they will be constrained by Monte Carlo comparisons with data.

\subsection{Preliminary Analysis Results}

The Q-weak experiment has finished its commissioning runs and first data-taking run cycle. This section gives the status of ongoing analyses of the beam polarization, helicity correlated beam prop- 
erty corrections, backgrounds, transverse asymmetry and raw asymmetries from the initial data analysis at the time of writing.

\subsubsection{Helicity Correlated Beam Property Corrections}

The detector sensitivities to helicity correlated beam properties have been analyzed. An example can be seen in Fig. 6.6, in which the beam properties, including positions $x$, $y$, directions $x^{\prime}, y^{\prime}$, energy $E$ and charge asymmetry $A_{q}$, are extracted from natural beam motion in a subset of runs. The sensitivities of $A_{m d a l l}$ (the asymmetry for the combination of all the Q-weak main detectors) versus run numbers are plotted. In this analysis, beam position and direction changes at target are extrapolated from BPM projections, while the energy difference is determined by the combination of information from a particular BPM $-\operatorname{bpm} 3 \mathrm{C} 12 \mathrm{X}, x$, and $x^{\prime}$. The energy sensitivity is around $-4 \mathrm{ppm} / \mathrm{ppm}$, and the sensitivity to the charge asymmetry seems quite stable at about $0.012 \%$ per percent $(0.012 \%$ change of signal for $1 \%$ of charge asymmetry change). Fig. 6.7 shows the contributions to $A_{\text {mdall }}$ corresponding to the sensitivities in Fig. 6.6, and Fig. 6.8 shows the sum of all helicity correlated beam property corrections [131]. Various regression schemes based on different combinations of beam monitors are also being studied and compared to each other in order to optimize the Q-weak regression method. A summary of helicity correlated beam properties for Q-weak Run I data is given in Table 6.3. The data were taken from the Pass-1 database, in which the energy information was not available; therefore, no average energy was evaluated in the table. The average values for other beam properties, beam charge asymmetries, position and angle differences at target are either within or close to the proposed specifications [132].

\subsubsection{Beam Polarization}

Beam polarization is one of the major error sources because $A_{\text {physics }}=A_{\text {experimental }} / P_{\text {beam }}$. The goal is to determine $P_{\text {beam }}$ to $1 \%$ using the Hall C Møller and Compton polarimeters. The Møller polarimeter provides invasive measurement to $0.75 \%$ accuracy at a few $\mu \mathrm{A}$, and the Compton polarimeter provides non-invasive and continuous measurement at full production beam current. 

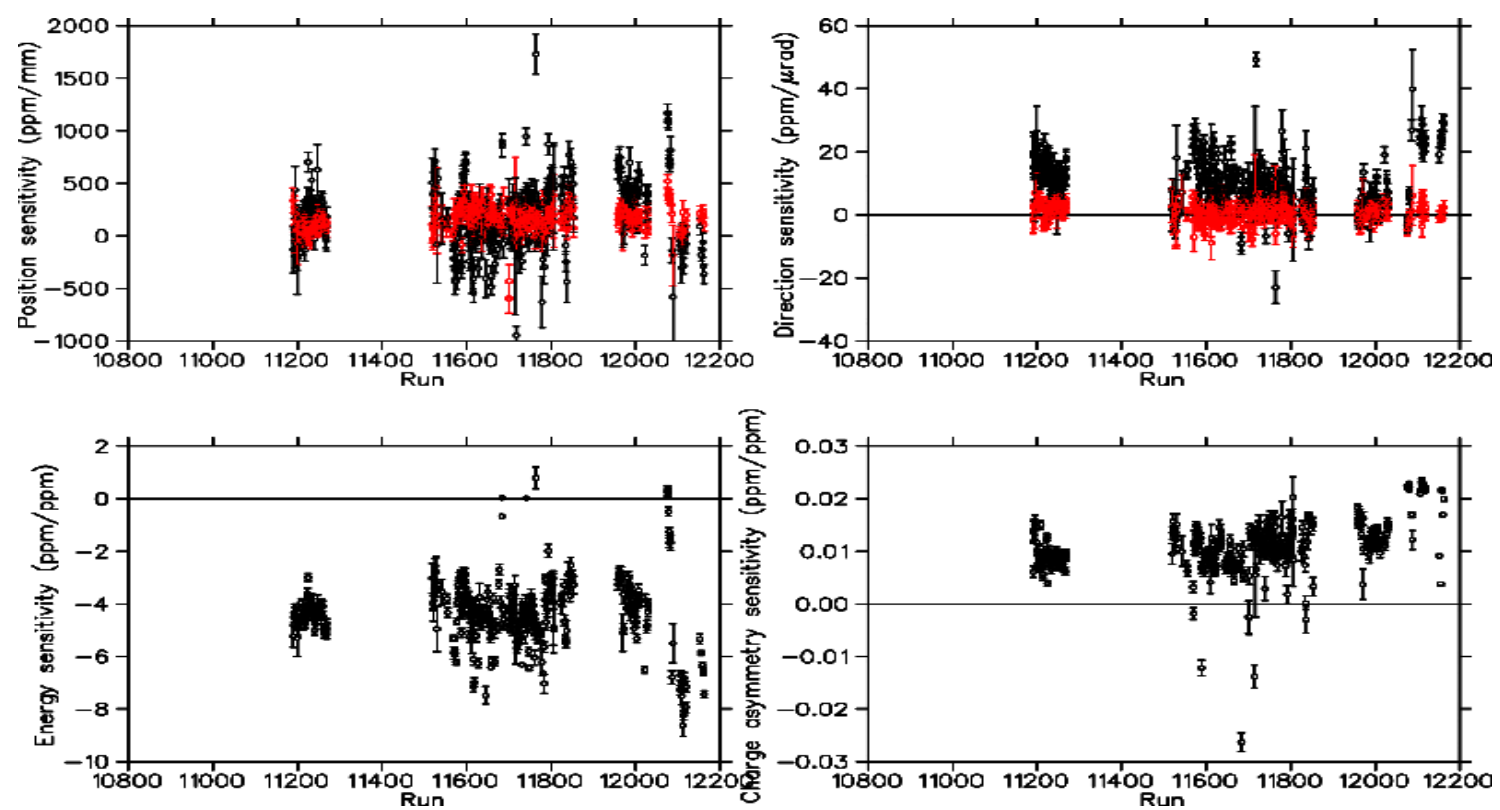

Figure 6.6: The main detector all bar sensitivities to beam motion $x$ (in black) and $y$ (in red), direction $\left(\theta_{x}, \theta_{y}\right)$ energy $(E)$ and charge asymmetry $\left(A_{q}\right)$ [131].
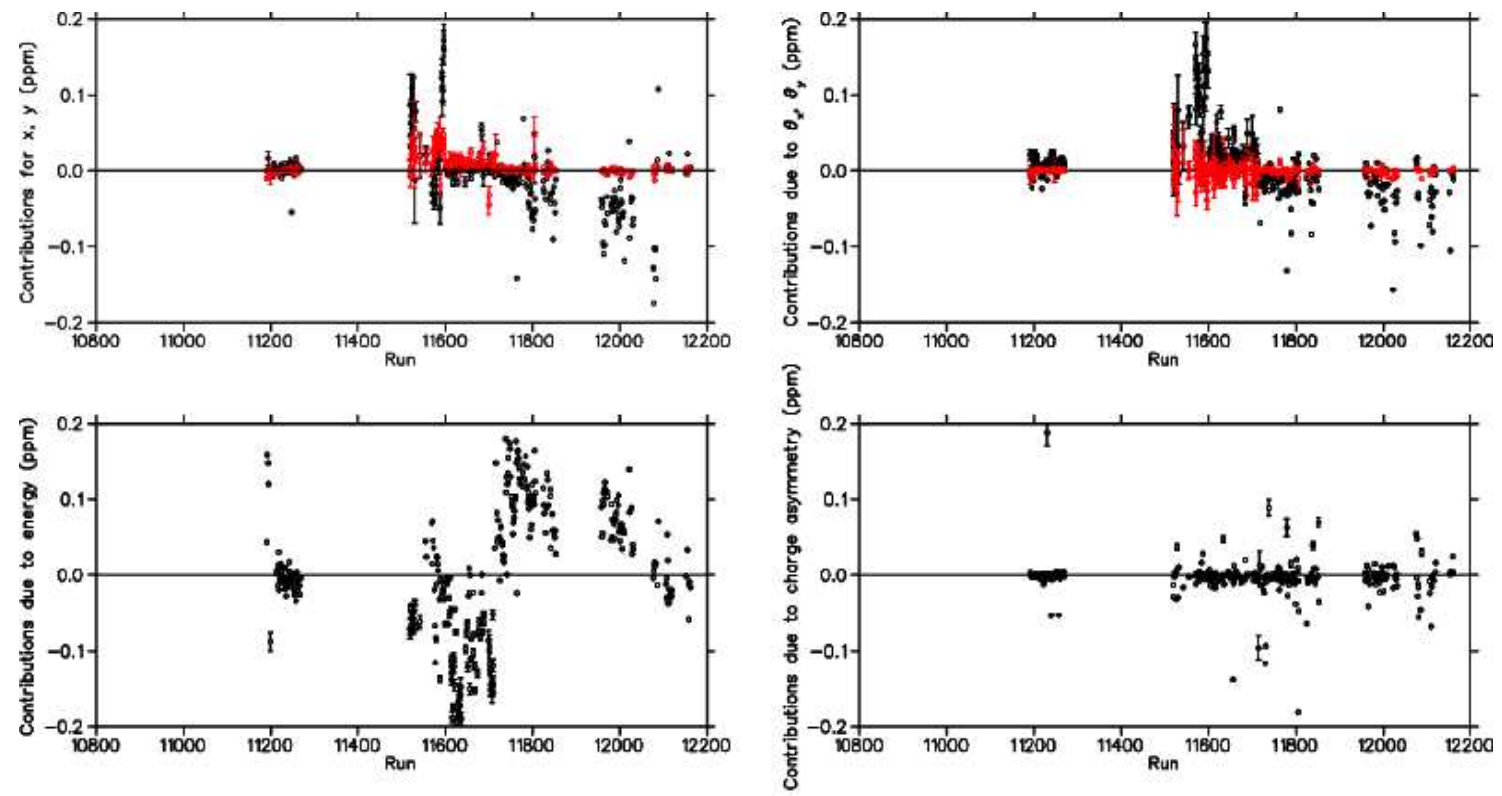

Figure 6.7: Helicity correlated beam property corrections to the asymmetry [131]. 


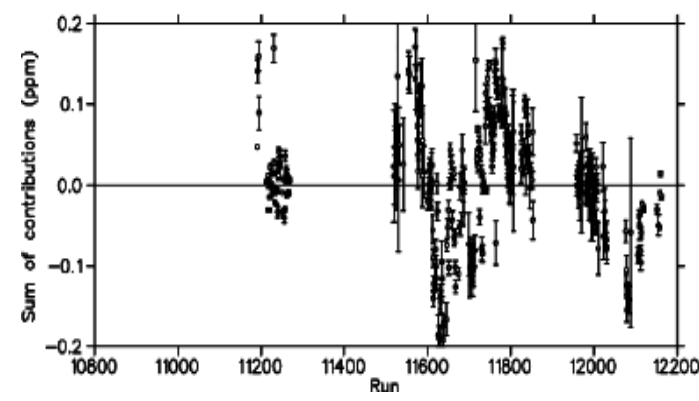

Figure 6.8: Sum of all helicity correlated beam property corrections to the asymmetry [131].

\begin{tabular}{|c|c|c|c|c|c|}
\hline & $\begin{array}{l}\text { IHWP } \\
\text { IN/OUT }\end{array}$ & $\begin{array}{l}\text { Wien } \\
\text { Left }\end{array}$ & $\begin{array}{l}\text { Wien } \\
\text { Right }\end{array}$ & $\begin{array}{c}\text { Run I } \\
\text { Average }\end{array}$ & $\begin{array}{c}\text { Proposal } \\
(2544 \mathrm{hrs})\end{array}$ \\
\hline $\begin{array}{c}\left\langle A_{q}\right\rangle \\
(\mathrm{ppm})\end{array}$ & $\begin{array}{c}\text { IN } \\
\text { OUT }\end{array}$ & $\begin{array}{r}-0.10 \pm 0.03 \\
0.21 \pm 0.03\end{array}$ & $\begin{array}{l}0.004 \pm 0.03 \\
-0.14 \pm 0.03\end{array}$ & $0.0083 \pm 0.015$ & $<0.1$ \\
\hline $\begin{array}{c}\langle\operatorname{tgt} X\rangle \\
(\mathrm{nm})\end{array}$ & $\begin{array}{l}\text { IN } \\
\text { OUT }\end{array}$ & $\begin{array}{r}-31 \pm 1 \\
17 \pm 1\end{array}$ & $\begin{array}{c}1.6 \pm 0.8 \\
14.3 \pm 0.8\end{array}$ & $3.9 \pm 0.5$ & $<2$ \\
\hline $\begin{array}{c}\langle\operatorname{tgt} Y\rangle \\
(\mathrm{nm})\end{array}$ & $\begin{array}{c}\text { IN } \\
\text { OUT }\end{array}$ & $\begin{array}{r}4 \pm 1 \\
-1 \pm 1\end{array}$ & $\begin{array}{r}-36 \pm 1 \\
13 \pm 1\end{array}$ & $-5.7 \pm 0.5$ & $<2$ \\
\hline $\begin{array}{c}\left\langle\operatorname{tgt} X^{\prime}\right\rangle \\
(\text { nrad })\end{array}$ & $\begin{array}{l}\text { IN } \\
\text { OUT }\end{array}$ & $\begin{array}{r}-1.18 \pm 0.04 \\
0.63 \pm 0.04\end{array}$ & $\begin{array}{c}-0.72 \pm 0.03 \\
0.6 \pm 0.03\end{array}$ & $-0.01 \pm 0.016$ & $<30$ \\
\hline $\begin{array}{l}\left\langle\operatorname{tgt} Y^{\prime}\right\rangle \\
(\mathrm{nrad})\end{array}$ & $\begin{array}{c}\text { IN } \\
\text { OUT }\end{array}$ & $\begin{array}{r}-0.2 \pm 0.03 \\
0.10 \pm 0.03\end{array}$ & $\begin{array}{r}-0.79 \pm 0.03 \\
0.83 \pm 0.03\end{array}$ & $-0.0024 \pm 0.016$ & $<30$ \\
\hline
\end{tabular}

Table 6.3: Summary of helicity correlated beam properties for the Q-weak Run I [132].

Beam polarization has been measured periodically over the entire Run I period. Fig. 6.9 shows some of the measured results, including Møller measurements and Compton measurements based on the electron detector alone. From the figure, the beam polarization has been typically between $86 \%$ and $88 \%$, better than the proposed $85 \%$. The Møller measurements typically have statistical uncertainty of less than $0.4 \%$ and systematic uncertainty of less than $0.6 \%$ [65], and the Compton measurements typically have about $1 \%$ statistical uncertainty and $0.6 \%$ partially evaluated systematic uncertainty [133]; the final systematics should be less than 1\% with Monte Carlo studies [134]. 


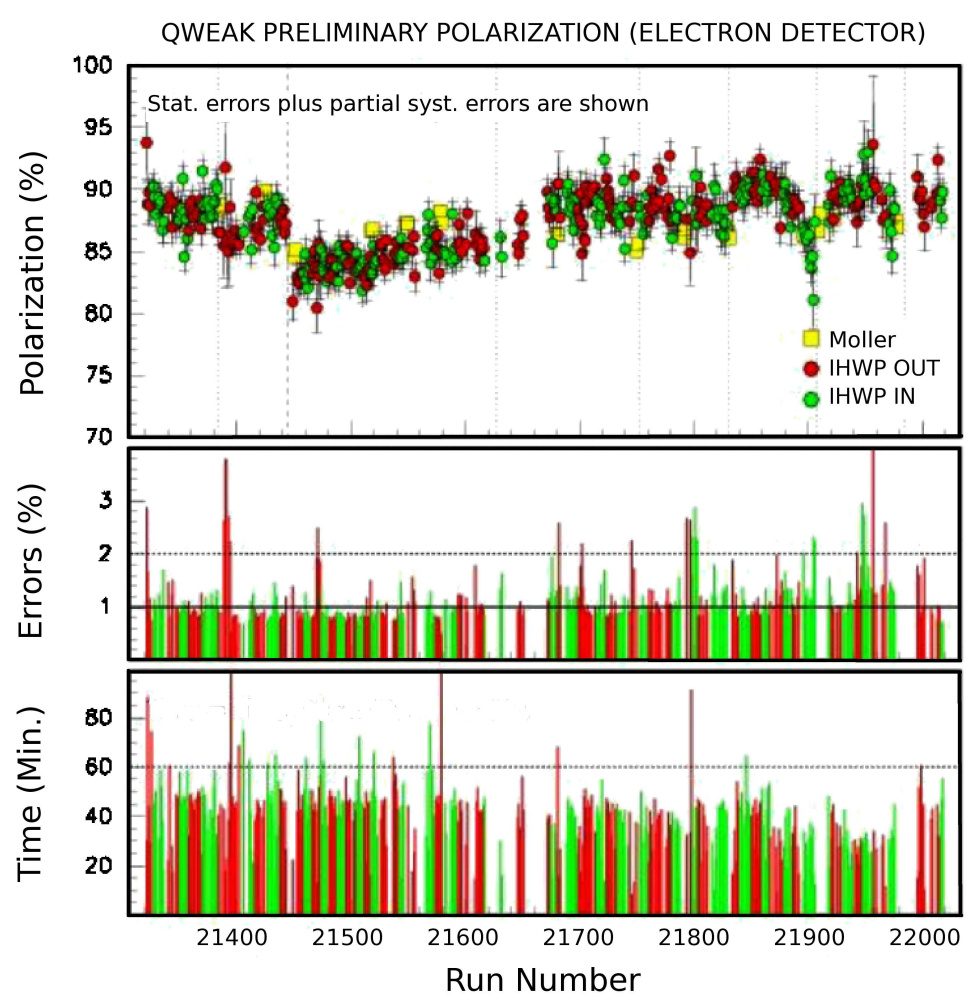

Figure 6.9: Preliminary measured beam polarization over runs with the Møller and Compton polarimeters [133].

These uncertainty estimates are very preliminary.

\subsubsection{Backgrounds}

As mentioned in Section 6.3.2, dilutions and asymmetries contributed by backgrounds need to be known for asymmetry corrections. In order to determine the dominant background contributions, the dilutions due to aluminum target windows and inelastic events, several dedicated measurements were made during Q-weak Run I. Table 6.4 lists the preliminary background dilution and correction results [129].

Apart from the background contributions from the aluminum target windows and inelastic events, there are diffuse backgrounds from the beamline. During the commissioning period, this 


\begin{tabular}{c|cc}
\hline \hline Source & Dilution of signal & Correction to asymmetry \\
\hline $\begin{array}{c}\text { Aluminum target windows } \\
\text { (elastics and quasi-elastics) }\end{array}$ & $3 \%$ & $20 \%$ \\
\hline $\begin{array}{c}\text { Inelastic asymmetry } \\
(N \rightarrow \Delta)\end{array}$ & $0.1 \%$ & $1 \%$ \\
\hline \hline
\end{tabular}

Table 6.4: Preliminary background dilution and correction results for the Q-weak Run I [135].

type of soft background contributed an $O(10) \%$ dilution of the main detector signal. After the installation of $2 \mathrm{~cm}$ thick lead pre-radiators in front of the main detectors, these contributions were reduced to $O(1) \%$. Following further improvements of the beamline shielding, such as plugging three shielding gaps and installing donut-shaped lead shields around the beam pipe near Region 2, the beamline backgrounds were reduced to $\sim 0.2 \%$ [129]. Systematic errors are under evaluation at this time.

\subsubsection{Transverse Asymmetry}

As discussed in Section 6.3.3, the residual transverse polarization, and the broken azimuthal symmetry in the detector system due to small differences between the individual main detectors may lead to a false asymmetry, which needs to be corrected knowing the measured transverse asymmetry $A_{T}$. During Q-weak Run I, a set of dedicated transverse asymmetry measurements for the $\mathrm{LH}_{2}$ target and the $4 \%$ DS Al target with fully vertically polarized beam was taken. To extract the transverse asymmetry, the regressed main detector asymmetries in eight octants were fitted to the function:

$$
A_{T}(\phi)=p_{0} \cos \left(\phi_{d e t}+p_{1}\right)+p_{2}
$$

where $\phi_{\text {det }}=($ octant -1$) \times 45^{\circ}$ is the average azimuthal angle, $p_{0}$ is the magnitude of the transverse asymmetry, $p_{1}$ is the phase offset to $\phi_{d e t}$, and $p_{2}$ involves the parity-violating asymmetry and background contributions. 
Preliminary results shown in Fig. 6.10 indicate good data quality. The fit changes sign with the IHWP state as it should; $p_{1}$ terms from the "IN" and "OUT" fits are different by $\sim 180^{\circ}$; and "IN+OUT" fits have a $p_{0}$ and a $p_{2}$ term that are consistent with zero. High statistical precision
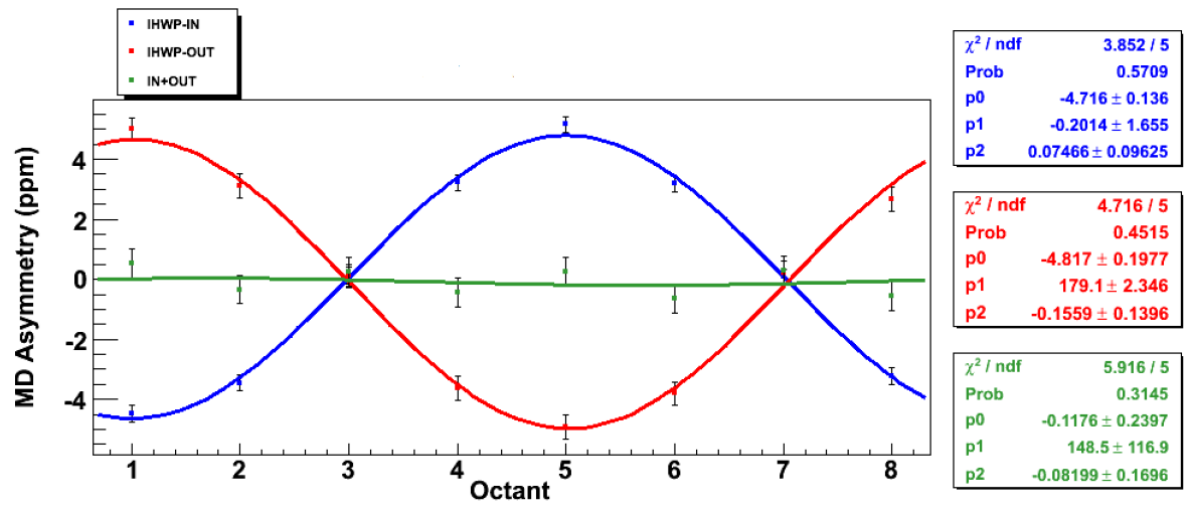

(a) $\mathrm{LH}_{2}$ target, regressed slug averages of main detector asymmetries
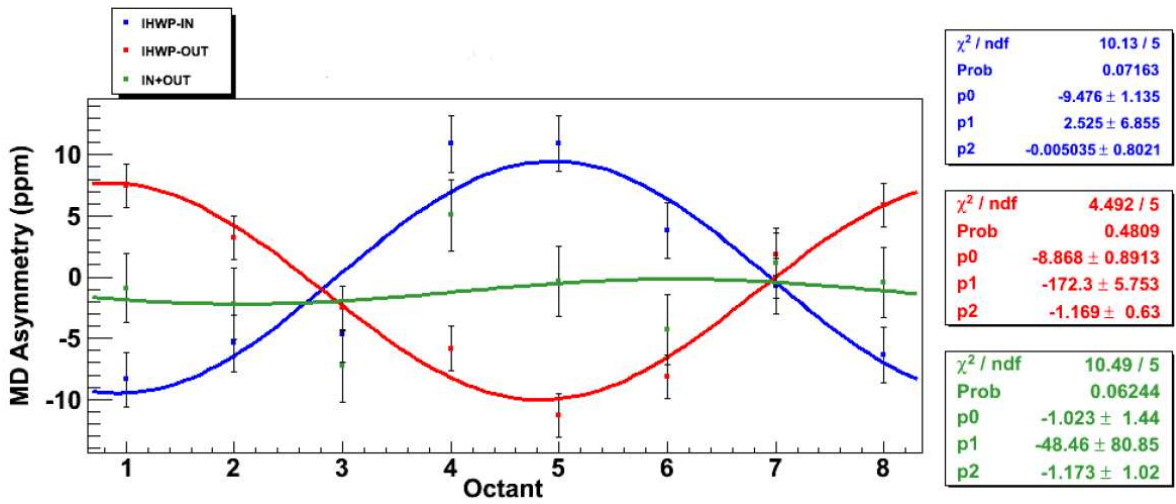

(b) $4 \% \mathrm{DS}$ aluminum target, regressed slug averages of main detector asymmetries

Figure 6.10: Preliminary results from transverse asymmetry measurements for $\mathrm{LH}_{2}$ target and $4 \%$ DS aluminum target [136].

on $A_{T}$ measurements has been achieved during Run I, with $2 \%$ for $\mathrm{LH}_{2}(-4.75 \pm 0.11 \mathrm{ppm})$, and $7.5 \%$ for the $4 \% \mathrm{DS} \mathrm{Al}$ target $(-9.1 \pm 0.7 \mathrm{ppm})$ [136]. These precisions are sufficient for the Q-weak transverse correction and target window background correction purposes. The measured transverse asymmetry will later be used to correct the Q-weak asymmetry for any residual transverse polarization components which may be of the order of a few percent. A first measurement of this with vertical polarization has been completed in Q-weak Run I. A set of horizontal transverse 
polarization data is planned to be taken during Run II.

\subsubsection{Raw Asymmetry}

The parity data accumulated during Q-weak Run I are summarized in Table 6.5. The data are grouped into 12 slugs (two for each Wien filter setting) according to the experimental configurations. The data in the Wien setting $0 / \mathrm{R}$ is for the first $25 \%$ measurement, which was assigned a different much larger blinding factor than the maximum $60 \mathrm{ppb}$ for all other slugs.

\begin{tabular}{ccrcrr}
\hline \hline $\begin{array}{c}\text { Wien } \\
(\# / F l i p)\end{array}$ & $\begin{array}{c}\text { Run } \\
\text { Range }\end{array}$ & $\begin{array}{r}\text { Slug } \\
\text { Range }\end{array}$ & $\begin{array}{c}\text { Date } \\
(2011)\end{array}$ & $\begin{array}{c}\text { Asymmetry [ppm] } \\
\text { (IHWP IN) }\end{array}$ & $\begin{array}{c}\text { Asymmetry [ppm] } \\
\text { (IHWP OUT) }\end{array}$ \\
\hline 0/R & $08964-09812$ & $<40$ & $13 / 01-10 / 02$ & $0.113 \pm 0.043$ & $-0.351 \pm 0.036$ \\
$1 / \mathrm{L}$ & $09939-10186$ & $42-58$ & $11 / 02-22 / 02$ & $-0.332 \pm 0.040$ & $0.214 \pm 0.044$ \\
2/R & $10196-11129$ & $59-80$ & $22 / 02-05 / 04$ & $0.106 \pm 0.031$ & $-0.229 \pm 0.034$ \\
$3 / \mathrm{L}$ & $11131-11390$ & $81-98$ & $05 / 04-14 / 04$ & $-0.227 \pm 0.038$ & $0.237 \pm 0.038$ \\
4/R & $11391-11711$ & $99-116$ & $14 / 04-28 / 04$ & $0.307 \pm 0.044$ & $-0.218 \pm 0.042$ \\
$5 / \mathrm{L}$ & $11714-11735$ & $117-136$ & $28 / 04-13 / 05$ & $-0.267 \pm 0.041$ & $0.087 \pm 0.034$ \\
\hline \hline
\end{tabular}

Table 6.5: Summary of Q-weak Run I asymmetry data (preliminary, blinded, and no correction for sign reversal) $[132,83]$.

Fig. 6.11 shows a plot of the asymmetry versus slug number during Q-weak Run I. It can be seen from the plot that the asymmetry sign reverses as the IHWP/Wien state changes. Therefore no significant systematic errors appear to be affecting the data. However, the average value of asymmetries from these Wien periods indicated poor internal consistency, especially for Wien period 5, in which the average asymmetry value showed a larger deviation from zero. Therefore, intense studies are being made to investigate the "IN+OUT" behavior for the Wien periods with large deviation [137]. To address this issue, further linear regression work is in progress to make detailed comparison of corrections from beam modulation and natural beam motion for a variety of independent variables. After the next pass analysis is complete, the global plots of unregressed and regressed asymmetries versus Wien state will be revisited. 
Asymmetry vs. Wien Period (Q-weak Run I, Blinded)

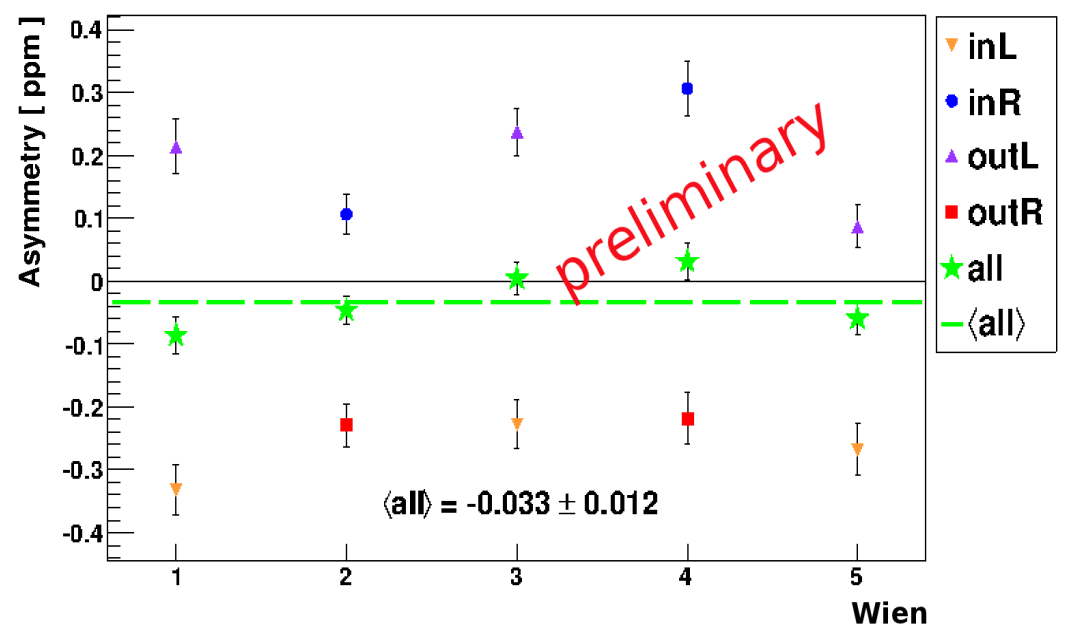

Figure 6.11: Blinded parity-violating asymmetries for all data with five Wien Filter settings (except the $25 \%$ data for Wien setting 0) $[132,83]$. The green dashed line represents the total average for each sign reversal configuration (IHWP IN and OUT, Wien Left and Right). The uncertainties shown are statistical.

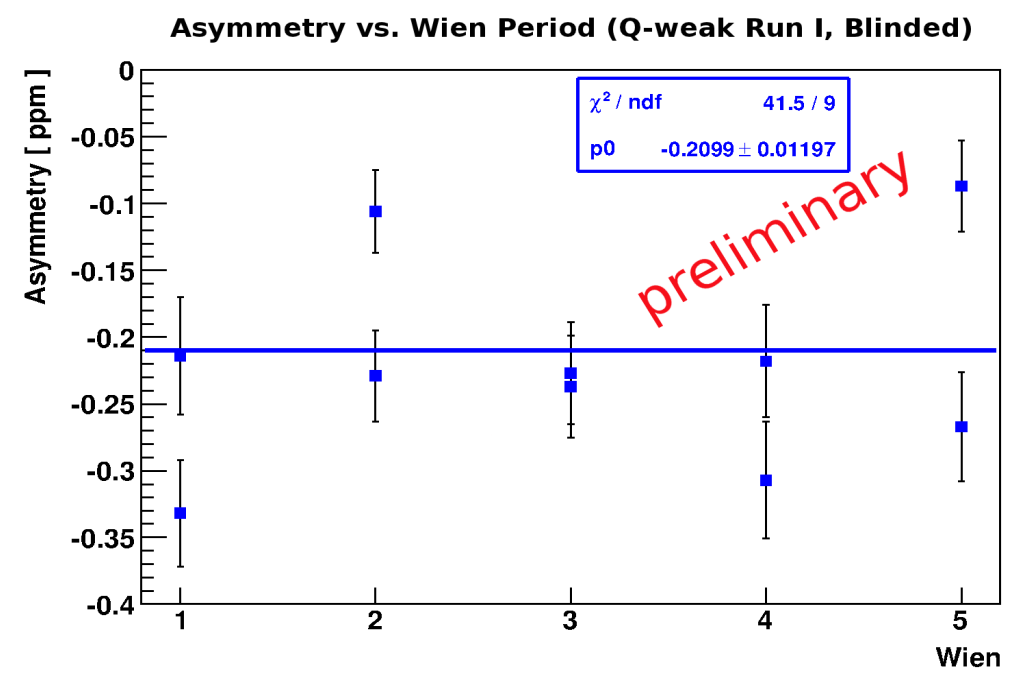

Figure 6.12: Total average of asymmetries for Wien period 1 - 5 (blinded). The line indicates the mean value of the data in these Wien periods. There are 2 sign-corrected data points for each Wien period, corresponding to IHWP IN and OUT configurations. 
After making sign corrections, the measured asymmetries from the slug data (not normalized to beam polarization) are plotted in Fig. 6.12. Naively, the mean value of Wien period 1-5 data is found to be

$$
A_{\text {meas }}(\text { Wien period } 1-5)=-0.210(\text { blinded }) \pm 0.012 \text { (stat.) } \mathrm{ppm} \text {. }
$$

This result has been blinded, containing a maximum blinding factor of $0.06 \mathrm{ppm}$. The statistical uncertainty is about $5.7 \%$. No preliminary extraction of the proton's weak charge is meaningful at this time, because the asymmetry data are still blinded. The lack of internal consistency of the data represents the present status of the data set. It is the subject of intense scrutiny within the collaboration at this time. 


\section{Chapter 7}

\section{Qweak Status Summary and Conclusion}

The Q-weak experiment will make a precision measurement of the proton's weak charge. This will be used to extract $\sin ^{2} \theta_{W}$ at $Q^{2} \sim 0.026(\mathrm{GeV} / \mathrm{c})^{2}$ to a precision of $0.3 \%$. The project is on track to reach the experimental goal. The experiment started its first commissioning data taking in July 2010, which was completed in the fall of 2010. The first production data taking cycle spanned January 2011 to May 2011. During these running periods,

- all critical subsystems were commissioned;

- the accelerator and experimental apparatus were working well, though not all the time;

- $150-180 \mu \mathrm{A}$ beam was typically delivered with $86-88 \%$ polarization, exceeding the proposal requirements;

- the helicity correlated beam parameters were controlled to an acceptable level;

- beam-line backgrounds were reduced from $O(10) \%$ to $O(0.1) \%$;

- initial background asymmetries and signal dilutions were measured;

- several tracking runs were taken;

- production parity data were accumulated and the raw asymmetry was measured to about $5 \%$. 
Changes, extensions and optimizations have been made during the down time from May to November 2011. The experiment resumed production runs in November 2011 and will complete its second data taking cycle in May 2012. When completed, the experiment will provide the world's best measurement of $\sin ^{2} \theta_{W}$ at low energies.

My specific contributions to the experiment, as presented in this thesis, have related to the scanner and to tracking (for details see Chapters 3, 4, 5 and Appendix C). In Chapter 3, details on the scanner detector development were introduced, from the very first design, Monte Carlo simulations, to prototype construction and TRIUMF beam tests, then to the later scanner detector construction and installation at JLab, the following commissioning, until the first results. Some scanner data were acquired during the Q-weak commissioning and production Run I. The analysis results for those data were discussed in Chapter 5. The scanner was used to map out the variations of rate distribution from low to high beam current. It was verified to be able to work in both low and high beam currents, although deficiencies for very high-current running were observed, arising from large accidental coincidence rates. Some unexpected results were found from PMT singles rates and asymmetric rate maps measured at high beam currents. Although there are still mysteries, suggestions on how to investigate these problems have been made. Methods for $\left\langle Q^{2}\right\rangle$ extrapolation with the scanner were discussed as well. The uncertainty of the $\left\langle Q^{2}\right\rangle$ extrapolation is dominated by systematics arising due to rate corrections. The extrapolated $\left\langle Q^{2}\right\rangle$ at high beam current agrees with the light-weighted $\left\langle Q^{2}\right\rangle$ determined by tracking runs at low current to a level of better than $0.1 \%$, which is far smaller than the $\left\langle Q^{2}\right\rangle$ shift of about $1.4 \%$ due to light-weighting in the main detectors.

Based on a magnetic field swimming algorithm, tracking software was developed for $Q^{2}$ determination. The software incorporates Q-weak track reconstruction and Monte Carlo simulation. This software has been used to obtain the first results on $\left\langle Q^{2}\right\rangle$, which are very encouraging (see Chapter 4). The code should allow a determination of $\left\langle Q^{2}\right\rangle$ to $\sim 0.5 \%$ if the detector specifications are met. Work to further improve track finding efficiency and track parameter resolution is currently underway. With more tracking data being taken in Q-weak Run II, $\left\langle Q^{2}\right\rangle$ is expected to be determined to its anticipated precision. 
As of completing this writing, some of the scanner Run I data have not been fully understood. The remaining mysteries will require further investigation during Q-weak Run II. Improvements on scanner detector performance are essential to provide accurate interpretation of the data. These can be done by improving detector shielding, optimizing its electronics chain and testing the linearity of the PMT rate response. The deadtime correction technique introduced in Chapter 5 also needs to be confirmed with Run II data, along with the Monte Carlo simulations of deadtime effects.

One of the scanner goals is to extrapolate the tracking results to $180 \mu \mathrm{A}$ full beam current, which was not achieved in the Q-weak Run I. The scanner was operated up to $165 \mu \mathrm{A}$ at that time. This therefore necessitates further investigation of rate distributions with the scanner at beam currents of $165-180 \mu \mathrm{A}$ during the Q-weak Run II. To perform $\left\langle Q^{2}\right\rangle$ extrapolation, some essential tests are required, such as the test of scanner linearity versus beam current. It is especially crucial to have a full bar calibration of the scanner detector against the VDC in the same octant, in order to complete the discussion on $\left\langle Q^{2}\right\rangle$ extrapolation method presented in Chapter 5. These measurements must also be completed during Q-weak Run II. 


\section{Bibliography}

[1] Qweak-Collaboration. http://www.jlab.org/qweak/.

[2] JLab. Thomas Jefferson National Accelerator Facility, USA. http://www.jlab.org/.

[3] S.A. Page et al. The Qweak Experiment at Jefferson Laboratory. In K. de Jager et al., editors, From Parity Violation to Hadronic Structure and More. Springer, 2006.

[4] S. Weinberg. A Model of Leptons. Physical Review Letters, 19(21):1264, 1967.

[5] A. Salam. Weak and Electromagnetic Interactions. In 8th Nobel Symposium on 'Elementary particle theory, relativistic groups and analyticity' edited by N. Svartholm, pages 367-377, 1969.

[6] S.L. Glashow, J. Iliopoulos, and L. Maiani. Weak Interactions with Lepton-hadron Symmetry. Physical Review D, 2(7):1285, 1970.

[7] P. Langacker. The Standard Model and Beyond. High Energy Physics, Cosmology and Gravitation. Taylor \& Francis, December 2009.

[8] B. Povh, K. Rith, and C. Scholz. Particles and Nuclei: An Introduction to the Physical Concepts. Springer Verlag, 2008.

[9] K. Nakamura et al. Review of Particle Physics. Journal of Physics G: Nuclear and Particle Physics, 37:075021, 2010. 
[10] T. Morii, C.S. Lim, and S.N. Mukherjee. The Physics of the Standard Model and Beyond. World Scientific Publishing Co. Pte. Ltd., 2004.

[11] B.R. Martin and G. Shaw. Particle physics. John Wiley \& Sons Inc, 2008.

[12] E.D. Commins and P.H. Bucksbaum. Weak Interactions of Leptons and Quarks. Cambridge University Press, Cambridge, July 1983.

[13] M.J. Musolf, T.W. Donnelly, J. Dubach, S.J. Pollock, S. Kowalski, and E.J. Beise. Intermediate-energy Semileptonic Probes of the Hadronic Neutral Current. Physics Reports, 239(1-2):1-178, 1994.

[14] E.J. Beise, M.L. Pitt, and D.T. Spayde. The SAMPLE Experiment and Weak Nucleon Structure. Progress in Particle and Nuclear Physics, 54(1):289-350, 2005.

[15] J. Erler, A. Kurylov, and M. J. Ramsey-Musolf. Weak Charge of the Proton and New Physics. Physical Review D, 68(1):016006, Jul 2003.

[16] J. Erler and M.J. Ramsey-Musolf. Weak Mixing Angle at Low Energies. Physical Review D, 72(7):073003, Oct 2005.

[17] S.C. Bennett, C.E. Wieman, et al. Measurement of the $6 S \rightarrow 7 S$ Transition Polarizability in Atomic Cesium and an Improved Test of the Standard Model. Physical Review Letters, 82(12):2484-2487, Mar 1999.

[18] S.G. Porsev, K. Beloy, and A. Derevianko. Precision Determination of Electroweak Coupling from Atomic Parity Violation and Implications for Particle Physics. Physical Review Letters, 102(18):181601, 2009.

[19] G.P. Zeller et al. Precise Determination of Electroweak Parameters in Neutrino-Nucleon Scattering. Physical Review Letters, 88(9):091802, Feb 2002.

[20] P.L. Anthony et al. Precision Measurement of the Weak Mixing Angle in Møller Scattering. Physical Review Letters, 95(8):081601, Aug 2005. 
[21] G. Abbiendi et al. Precise Determination of the Z Resonance Parameters at LEP: 'Zedometry'. European Physical Journal, C19:587-651, 2001.

[22] K. Abe et al. High-Precision Measurement of the Left-Right $Z$ Boson Cross-Section Asymmetry. Physical Review Letters, 84(26):5945-5949, Jun 2000.

[23] J.T. Londergan and A.W. Thomas. Implications of Current Constraints on Parton Charge Symmetry. Journal of Physics G: Nuclear and Particle Physics, 31:1151, 2005.

[24] Moller-Collaboration. An Ultra-precise Measurement of the Weak Mixing Angle using Møller Scattering. http://hallaweb.jlab.org/12GeV/Moller/, December 2008.

[25] F. Halzen and A.D. Martin. Quarks and Leptons: Introductory Course in Modern Particle Physics. John Wiley \& Sons, international edition, December 1984.

[26] R.G. Sachs. Nucleon Electromagnetic Form Factors at High Momentum Transfer. Physical Review Letters, 12(9):231-233, Mar 1964.

[27] R.P. Feynman. Photon Hadron Interactions (W.A. Benjamin, New York), 1972.

[28] J.A. Tjon, P.G. Blunden, and W. Melnitchouk. Detailed Analysis of Two-boson Exchange in Parity-Violating ep Scattering. Physical Review C, 79(5):055201, 2009.

[29] M.N. Rosenbluth. High Energy Elastic Scattering of Electrons on Protons. Physical Review, 79(4):615, 1950.

[30] I.A. Qattan et al. Precision Rosenbluth Measurement of the Proton Elastic Form Factors. Physical Review Letters, 94(14):142301, Apr 2005.

[31] E. Reya and K. Schilcher. Neutral-current Effects in Elastic Electron-Nucleon Scattering. Physical Review D, 10(3):952, 1974.

[32] D.H. Beck and R.D. McKeown. Parity-Violating Electron Scattering and Neucleon Structure. Arxiv preprint hep-ph/0102334, 2001. 
[33] W.J. Marciano and A. Sirlin. Some General Properties of the $O(\alpha)$ Corrections to Parity Violation in Atoms. Physical Review D, 29(1):75-88, Jan 1984.

[34] Qweak-Collaboration. The Qweak Experiment: A Search for New Physics at the TeV Scale via a Measurement of the Proton's Weak Charge, Proposal JLab E02-020. http://www.jlab.org/qweak/, December 2007.

[35] P.A. Souder et al. Measurement of Parity Violation in the Elastic Scattering of Polarized Electrons from C12. Physical Review Letters, 65(6):694-697, Aug 1990.

[36] A. Acha, K. A. Aniol, D.S. Armstrong, J. Arrington, T. Averett, S.L. Bailey, J. Barber, A. Beck, H. Benaoum, J. Benesch, et al. Precision Measurements of the Nucleon Strange Form Factors at $Q^{2} \sim 0.1 \mathrm{GeV}^{2}$. Physical Review Letters, 98(3):32301, 2007.

[37] D.S. Armstrong, J. Arvieux, R. Asaturyan, T. Averett, S.L. Bailey, G. Batigne, D.H. Beck, E.J. Beise, J. Benesch, L. Bimbot, et al. Strange-quark Contributions to Parity-Violating Asymmetries in the Forward G0 Electron-proton Scattering Experiment. Physical Review Letters, 95(9):92001, 2005.

[38] F.E. Maas, P. Achenbach, K. Aulenbacher, S. Baunack, L. Capozza, J. Diefenbach, K. Grimm, Y. Imai, T. Hammel, D. Von Harrach, et al. Measurement of Strange-Quark Contributions to the Nucleon's Form Factors at $Q^{2}=0.230(\mathrm{GeV} / \mathrm{c})^{2}$. Physical Review Letters, 93(2):22002, 2004.

[39] F.E. Maas, K. Aulenbacher, S. Baunack, L. Capozza, J. Diefenbach, B. Gläser, T. Hammel, D. Von Harrach, Y. Imai, et al. Evidence for Strange-Quark Contributions to the Nucleon's Form Factors at $Q^{2}=0.108(\mathrm{GeV} / \mathrm{c})^{2}$. Physical Review Letters, 94(15):152001, 2005.

[40] R.D. Young, J. Roche, R.D. Carlini, and A.W. Thomas. Extracting Nucleon Strange and Anapole Form Factors from World Data. Physical Review Letters, 97(10):102002, 2006. 
[41] R.D. Young, R.D. Carlini, A.W. Thomas, and J. Roche. Testing the Standard Model by Precision Measurement of the Weak Charges of Quarks. Physical Review Letters, 99(12):122003, Sep 2007.

[42] S.L. Zhu, S.J. Puglia, B.R. Holstein, and M.J. Ramsey-Musolf. Nucleon Anapole Moment and Parity-Violating ep Scattering. Physical Review D, 62(3):033008, 2000.

[43] J. Schwinger. Quantum Electrodynamics. II. Vacuum Polarization and Self-Energy. Physical Review, 75:651-679, Feb 1949.

[44] W.J. Marciano. Precision Test of the Standard Electroweak Model. Advanced Series on Directions in High Energy Physics, 14(1):170, 2010.

[45] M. Gorchtein and C.J. Horowitz. Dispersion $\gamma Z$-Box Correction to the Weak Charge of the Proton. Physical Review Letters, 102(9):091806, Mar 2009.

[46] A. Sibirtsev, P.G. Blunden, W. Melnitchouk, and A.W. Thomas. $\gamma$-Z Corrections to ForwardAngle Parity-Violating ep Scattering. Physical Review D, 82(1):013011, Jul 2010.

[47] B.C. Rislow and C.E. Carlson. Contributions from $\gamma$ Z Box Diagrams to Parity Violating Elastic ep Scattering. Physical Review D, 83(11):113007, 2011.

[48] M. Gorchtein, C.J. Horowitz, and M.J. Ramsey-Musolf. Model Dependence of the $\gamma$-Z Dispersion Correction to the Parity-Violating Asymmetry in Elastic ep scattering. Physical Review C, 84:015502, Jul 2011.

[49] W.J. Marciano and A. Sirlin. Radiative Corrections to Atomic Parity Violation. Physical Review D, 27(3):552-556, Feb 1983.

[50] P.G. Blunden, W. Melnitchouk, and A.W. Thomas. New Formulation of $\gamma$-Z Box Corrections to the Weak Charge of the Proton. Physical Review Letters, 107:081801, Aug 2011.

[51] T. Mossberg, A. Flusberg, and SR Hartmann. Optical Second-Harmonic Generation in Atomic Thallium Vapor. Optics Communications, 25(1):121-124, 1978. 
[52] C.Y. Prescott, W.B. Atwood, RLA Cottrell, H. Destaebler, E.L. Garwin, A. Gonidec, R.H. Miller, L.S. Rochester, T. Sato, D.J. Sherden, et al. Further Measurements of Parity Nonconservation in Inelastic Electron Scattering. Physics Letters B, 84(4):524-528, 1979.

[53] W. Heil, J. Ahrens, HG Andresen, A. Bornheimer, D. Conrath, K.J. Dietz, W. Gasteyer, H.J. Gessinger, W. Hartmann, J. Jethwa, et al. Improved Limits on the Weak, Neutral, Hadronic Axial Vector Coupling Constants from Quasielastic Scattering of Polarized Electrons. Nuclear Physics B, 327(1):1-31, 1989.

[54] Qweak-Collaboration. The Qweak Experiment: A Search for New Physics at the TeV Scale via a Measurement of the Proton's Weak Charge, Proposal JLab E02-020. http://www.jlab.org/qweak/, December 2004.

[55] Qweak-Collaboration. The Qweak Experiment: A Search for New Physics at the TeV Scale via a Measurement of the Proton's Weak Charge, Proposal JLab E02-020. http://www.jlab.org/qweak/, December 2001.

[56] J. M. Grames. Measurement of a Weak Polarization Sensitivity to the Beam Obit of the CEBAF Accelerator. $\mathrm{PhD}$ thesis, University of Illinois at Urbana-Champaign, 2000.

[57] T. Maruyama, E.L. Garwin, R. Prepost, and G. H. Zapalac. Electron-spin Polarization in Photoemission from Strained GaAs Grown on GaAs1-xPx. Physical Review B, 46(7):42614264, Aug 1992.

[58] R. Prepost and T. Maruyama. Advances in Polarized Electron Sources. Annual Review of Nuclear and Particle Science, 45(1):41-88, 1995.

[59] P. A. Adderley, J. Clark, J. Grames, J. Hansknecht, K. Surles-Law, D. Machie, M. Poelker, M. L. Stutzman, and R. Suleiman. Load-locked DC High Voltage GaAs Photogun with an Inverted-geometry Ceramic Insulator. Physical Review Special Topics - Accelerators and Beams, 13:010101, Jan 2010. 
[60] T. Maruyama, D.-A. Luh, A. Brachmann, J.E. Clendenin1, E.L. Garwin, S. Harvey, J. Jiang, R.E. Kirby, C.Y. Prescott, R. Prepost, A.M. Moy, et al. Systematic Study of Polarized Electron Emission from Strained GaAsGaAsP Superlattice Photocathodes. Applied Physics Letters, 85(13):2640, 2004.

[61] M. Pitt. Overview of Technical Challenges inParity-Violating Electron Scattering Experiments, PAVI09: 4th International Workshop "From Parity Violation to Hadronic Structure and more". Conference talk, June 2009.

[62] J. Grames, P. Adderley, J. Benesch, J. Clark, J. Hansknecht, R. Kazimi, D. Machie, and M. Poelker. Two Wien Filter Spin Flipper, 2011 Particle Accelerator Conference (PAC'11), New York, NY, 28 Mar - 1 Apr 2011. In JLAB-ACC-11-1377; DOE/OR/23177-1608, March 2011.

[63] B. Odom, D. Hanneke, B. D'Urso, and G. Gabrielse. New Measurement of the Electron Magnetic Moment Using a One-Electron Quantum Cyclotron. Physical Review Letters, 97(3):030801, July 2006.

[64] B. Steiner, W. Ackermann, W.F.O. Muller, and T. Weiland. Wien Filter as a Spin Rotator at Low Energy. In Particle Accelerator Conference, 2007. PAC. IEEE, pages 170-172. IEEE, 2007.

[65] D. Gaskell. Møller Polarimetry (Qweak Document 1350-v2). Technical report, JLab, February 2011.

[66] D. Gaskell, D. Meekins, and C. Yan. New Methods for Precision Møller Polarimetry. The European Physical Journal A - Hadrons and Nuclei, 32:561-564, 2007.

[67] J.W. Martin, D. Dutta, A. Narayan, and P. Wang. Diamond Detectors for Compton Polarimetry. AIP Conference Proceedings, 1182(1):623-626, 2009.

[68] D. Story. Compton Polarimetry. Honours Thesis. University of Winnipeg, May 2007. 
[69] G. Bardin, C. Cavata, D. Neyret, B. Frois, J. P. Jorda, J. M. Legoff, S. Platchkov, L. Steinmetz, M. Juillard, M. Authier, et al. Conceptual Design Report of a Compton Polarimeter for CEBAF Hall A. DAPNIA/SPhN-96-14, 1996.

[70] D.C. Jones et al. Compton Polarimetry Update (Qweak Document 1413-v1). Technical report, JLab, June 2011.

[71] A. Narayan. A Diamond Micro-strip Electron Detector for Compton Polarimetry, Qweak Document 1478-v2. Technical report, JLab, September 2011.

[72] N. Nuruzzaman. Full List of Beam Components of Hall-C Beamline for Q-weak Experiment (Qweak Document 1203-v3). Technical report, JLab, September 2010.

[73] R. Kazimi et al. Precision Intercomparison of Beam Current Monitors at CEBAF. In Particle Accelerator Conference, 1995., Proceedings of the 1995, volume 4, pages 2610-2612, 1995.

[74] W. Barry. A General Analysis of Thin Wire Pickups for High Frequency Beam Position Monitors. Nuclear Instruments and Methods in Physics Research, A301(3):407-416, 1991.

[75] T. Powers. Improvement of the Noise Figure of the CEBAF Switched Electrode Electronics BPM system. AIP Conference Proceedings, 451(1):256-265, 1998.

[76] C. Yan et al. Superharp - A Wire Scanner with Absolute Position Readout for Beam Energy Measurement at CEBAF. Nuclear Instruments and Methods in Physics Research, A365(23):261-267, 1995.

[77] J.-F. Rajotte. Achieving Our Statistical Goal (Qweak Document 1439-v1). Technical report, JLab, June 2011.

[78] M. Pitt, J. Leacock, and D. Mack. Halo Monitor Status (Qweak Document 1288-v2). Technical report, JLab, September 2010.

[79] C. Yan, N. Sinkine, and R. Wojcik. Linear Beam Raster for Cryogenic Targets. Nuclear Instruments and Methods in Physics Research, A539(1-2):1-15, 2005. 
[80] G. Smith. Qweak Liquid Hydrogen Target: Preliminary Design and Safety Document (Qweak Document 1041-v2). Technical report, JLab, August 2009.

[81] G. Smith. Qweak Target Measured Performance (Qweak Document 1481-v1). Technical report, JLab, September 2011.

[82] P. Wang. Magnetic field Simulation and Mapping for the Qweak Experiment. Master's thesis, University of Manitoba, Winnipeg, Canada, June 2007.

[83] P. Wang. A Measurement of the Proton's Weak Charge Using an Integration Cerenkov Detector System. PhD thesis, University of Manitoba, Winnipeg, Canada, August 2011.

[84] M. Pitt. Region 2 Chambers and Mini-Torus (Qweak Document 107-v1). Technical report, JLab, March 2004.

[85] G. Smith. Qweak Status (Qweak Document 1337-v1). Technical report, JLab, January 2011.

[86] S. Yang. $Q^{2}$ and Tracking Report (Qweak Document 1504-v1). Technical report, JLab, October 2011.

[87] K. Myers. Qweak Trigger Scintillator Specifications and Cosmic Tests (Qweak Document 1220-v1). Technical report, JLab, April 2010.

[88] J. Leacock. Qweak Luminosity Monitors - Characterization and Performance (Qweak Document 1395-v1). Technical report, JLab, May 2011.

[89] P. King. Data Acquisition/Online Analysis (Qweak Document 1274-v1). Technical report, JLab, August 2010.

[90] CODA. An Online Data Acquisition System Developed at JLab. http://coda.jlab.org/.

[91] B. Roberts. VME QWEAK 8-Channel 18-Bit ADC, Qweak Document 693-v1. Technical report, JLab, August 2007.

[92] CAEN. Costruzioni Apparecchiature Elettroniche Nucleari S.p.A. http://www.caen.it/. 
[93] P. King. DAQ and Analysis Status (Qweak Document 1166-v1). Technical report, JLab, February 2010.

[94] R.S. Hicks, P. Decowski, C. Arroyo, M. Breuer, J. Celli, E. Chudakov, K.S. Kumar, M. Olson, G.A. Peterson, K. Pope, et al. Flux Profile Scanners for Scattered High-energy Electrons. Nuclear Instruments and Methods in Physics Research, A553(3):470-482, 2005.

[95] K.A. Aniol, D.S. Armstrong, T. Averett, M. Baylac, E. Burtin, J. Calarco, G.D. Cates, C. Cavata, Z. Chai, C.C. Chang, et al. Parity-Violating Electroweak Asymmetry in ep Scattering. Physical Review C, 69(6):065501, 2004.

[96] P.L. Anthony et al. Observation of Parity Nonconservation in Møeller Scattering. Physical Review Letters, 92(18):181602, 2004.

[97] M. Frank and I.E. Tamm. Coherent Radiation of Fast Electrons. In Dokl. AN SSSR, volume 14, pages 109-112, 1937.

[98] D. Mack. A Quartz Cerenkov Detector (Qweak Document 6-v1). Technical report, JLab, March 2002.

[99] P. Medeiros. Private Communication, May 2009.

[100] S. Agostinelli, J. Allison, K. Amako, J. Apostolakis, H. Araujo, P. Arce, M. Asai, D. Axen, S. Banerjee, G. Barrand, et al. Geant4 - A Simulation Toolkit. Nuclear Instruments and Methods in Physics Research, A506(3):250-303, 2003.

[101] S. Agostinelli et al. GEANT4 Collaboration. Nuclear Instruments and Methods in Physics Research, A506(250):13, 2003.

[102] MIRO-4. ALANOD Aluminium-Veredlung GmbH and Co. KG, Ennepetal, Germany. Alzak is a registered trademark of the Aluminum Company of America (ALCOA). http://alanod.com/. 
[103] Photonis Technologies. http://www.photonis.com/.

[104] Lintech-Motion. 2D Linear Motion Ball-screw Assembly, 912102 and 91212 series for each axis. http://www.lintechmotion.com/.

[105] Danaher Motion. http://www.danahermotion.com.

[106] Galil Motion Control. http://www.galilmc.com/.

[107] Honeywell Sensing and Control. http://sensing.honeywell.com/.

[108] Position Sensor. A-Tech Instruments Ltd. http://www.a-tech.ca/.

[109] National Instrument. http://www.ni.com/.

[110] J. Pan. Scanner Alignment Report from JLab Survey Group, Subatomic Physics Elog at the University of Winnipeg. http://nuclear.uwinnipeg.ca/elog/Scanner/116, July 2010.

[111] J. Pan. Technical Drawings for Scanner, Subatomic Physics Elog at the University of Winnipeg. http://nuclear.uwinnipeg.ca/elog/scanner.

[112] P. Medeiros. Private Communication, July 2011.

[113] D. Mack. Private Communication, February 2011.

[114] W. Deconinck. Qweak Track Reconstruction Software Document (appearing as doxygen comments of the software), http://qweak.jlab.org/QwAnalysis_Docs/html/, 2009.

[115] W. Wander. Reconstruction of High Energy Scattering Events in the HERMES Experiment. PhD thesis, University of Erlangen-Nürnberg, University of Erlangen-Nürnberg, 1996.

[116] T. Pang. An Introduction to Computational Physics. Cambridge University Press, New York, September 1997.

[117] J. Pan. Momentum Determination in QTR (Qweak Document 1171-v1). Technical report, JLab, February 2010. 
[118] J. Pan. First Look at $Q^{2}$ by Bridging R2 and R3 Partial Tracks. Qweak Analysis and Aimulation Elog. https:/qweak.jlab.org/elog/Analysis+\%26+Simulation/191, February 2011.

[119] A. Asaturyan. Region-1 Sliders, "Passive" and "Active" Detector Status Update, Qweak Physics Meeting: November 17, 2011, https:/qweak.jlab.org/wiki/index.php/Meetings, November 2011.

[120] D.S. Armstrong. $Q^{2}$ and Tracking (Qweak Document 1429-v1). Technical report, JLab, June 2011.

[121] W.R. Leo. Techniques for Nuclear and Particle Physics Experiments. Springer-Verlag, 2nd edition, 1994.

[122] G. Faraci, S. Notarrigo, and A.R. Pennisi. Dead Time Corrections in Coincidence Measurements by Time-To-Pulse-Height Converters or Standard Coincidence Systems. Nuclear Instruments and Methods in Physics Research, 165:325-332, 1979.

[123] Photonis Voltage Dividers. http://www.photonis.com/.

[124] R. Mahurin. Main Detector Event-Mode Gains during Weekend Ending March 14. Qweak Detector Elog. https://qweak.jlab.org/elog/Detector/38, March 2011.

[125] K. Myers. Scanner maps on 4\% DS Aluminum and 1\% US Aluminum. Qweak Analysis and Simulation Elog. https://qweak.jlab.org/elog/Analysis+\%26+Simulation/351 .

[126] ROOT. http://root.cern.ch/drupal/.

[127] MySQL. http://www.mysql.com/.

[128] G. Smith. Qweak Status (Qweak Document 1410-v3). Technical report, JLab, June 2011.

[129] D. Mack. Measuring the Proton's Weak Charge (Qweak Document 1466-v1). Technical report, JLab, August 2011. 
[130] D. Androic, D.S. Armstrong, J. Arvieux, S.L. Bailey, D.H. Beck, E.J. Beise, J. Benesch, F. Benmokhtar, L. Bimbot, J. Birchall, et al. Transverse Beam Spin Asymmetries at Backward Angles in Elastic Electron-Proton and Quasielastic Electron-Deuteron Scattering. Physical Review Letters, 107(2):22501, 2011.

[131] J. Birchall. Sample of Updated Sensitivities. Qweak Analysis and Simulation Elog. https://qweak.jlab.org/elog/Analysis+\%26+Simulation/362, May 2011.

[132] J.-F. Rajotte. Helicity-correlated Beam Properties (Qweak Document 1419-v2). Technical report, JLab, June 2011.

[133] V. Tvaskis. Electron Detector (Qweak Document 1415-v1). Technical report, JLab, August 2011.

[134] J. Martin. Private Communication, November 2011.

[135] W. Deconinck. The Qweak Experiment: A Search for New Physics at the TeV Scale (Qweak Document 1487-v1). Technical report, JLab, September 2011.

[136] B. Waidyawansa. Preliminary Results from the Transverse Measurement (Qweak Document 1414-v6). Technical report, JLab, August 2011.

[137] M. Pitt. Slow Reversal Behavior of the Elastic Data - Brief Review (Qweak Document 1473-v1). Technical report, JLab, August 2011. 


\title{
Appendix A
}

\section{Engineering Drawing of the 2D Motion}

\author{
Assembly
}




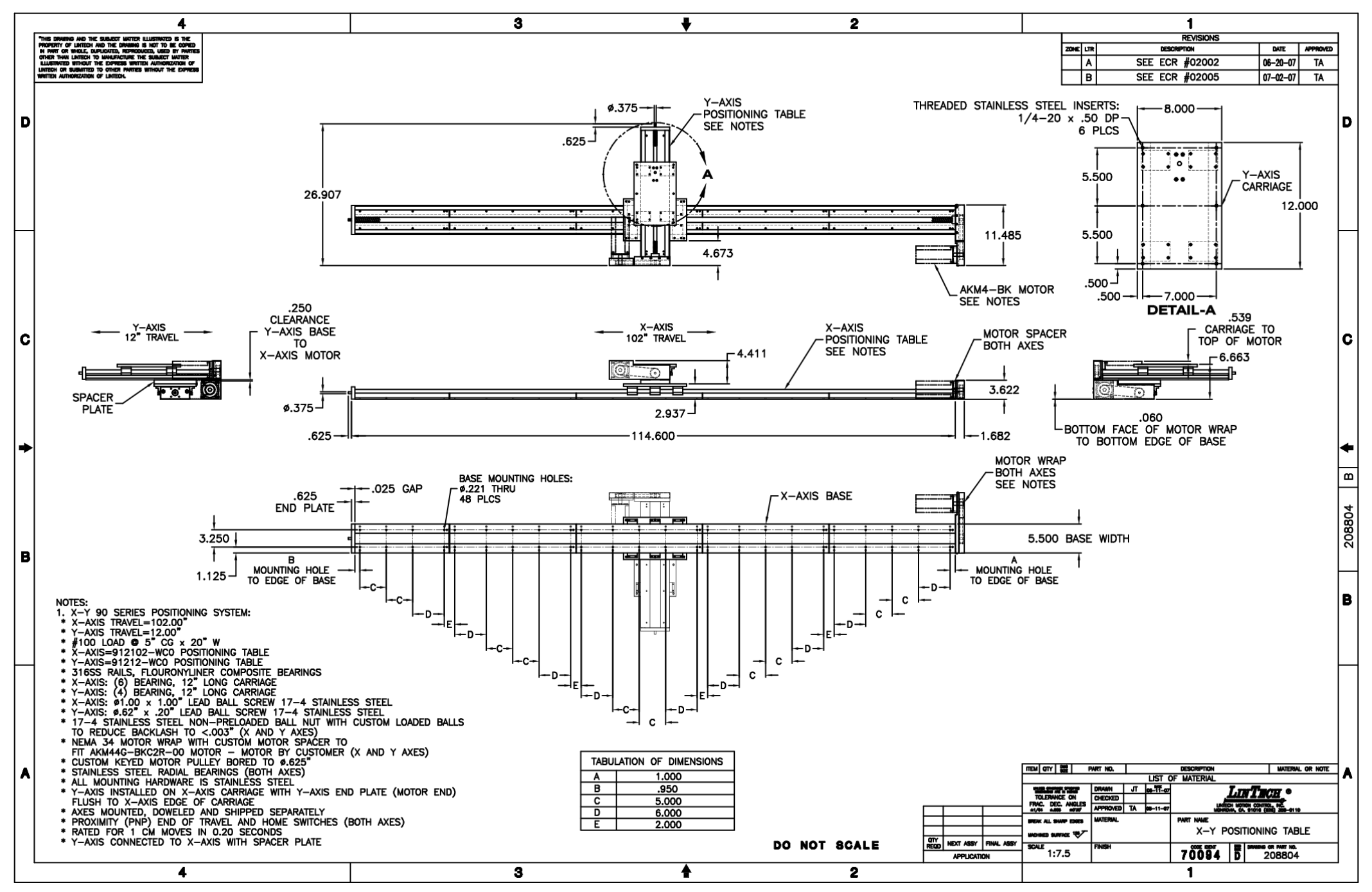

Figure A.1: Engineering drawing of Lintech 2D motion assembly. 


\section{Appendix B}

\section{Scanner Run List}

Table B.1 lists the scanner runs, taken during the Q-weak Run I. Only a small number of runs were taken due to limited beam time. Therefore, these runs are focused on the main purpose of the scanner

$-\left\langle Q^{2}\right\rangle$ extrapolation, with several runs for scanner performance study. Several normalized scanner rate maps (see Section 5.1 for the discussion of beam current normalization) taken at different beam currents are also shown in Fig. B.1 - Fig. B.5 for reference, where the color scale indicates rate in units of $\mathrm{Hz} / \mu \mathrm{A}$. 


\begin{tabular}{|c|c|c|c|c|c|c|c|c|}
\hline Run \# & $\begin{array}{l}\mathrm{I}_{\text {beam }} \\
(\mu \mathrm{A})\end{array}$ & Target & $\begin{array}{l}\text { QTOR Current } \\
\text { (A) }\end{array}$ & $\begin{array}{l}\text { Position Measured } \\
\text { by Module }\end{array}$ & $\begin{array}{l}\text { Disc. Pulse } \\
\text { Width (ns) }\end{array}$ & $\begin{array}{c}\text { Delay } \\
\text { line }(n s)\end{array}$ & Time & Comment \\
\hline 6615 & 10 & $\mathrm{LH}_{2}$ & 8921 & QDC & 40 & 64 & $11 / 06 / 2010$ & \\
\hline 6616 & 50 & $\mathrm{LH}_{2}$ & 8921 & QDC & 40 & 64 & $11 / 06 / 2010$ & \\
\hline 9710 & 145 & $\mathrm{LH}_{2}$ & 8921 & QDC & 40 & 64 & $2 / 04 / 2011$ & bad halo \\
\hline 10044 & 150 & $\mathrm{LH}_{2}$ & 8921 & VQWK & 40 & 64 & $2 / 14 / 2011$ & \\
\hline 10897 & 150 & $\mathrm{LH}_{2}$ & 8921 & VQWK & $5-40$ & 64 & $3 / 18 / 2011$ & parasitic run, DISC scan \\
\hline 10913 & 150 & $\mathrm{LH}_{2}$ & 8921 & VQWK & 10 & 64 & $3 / 19 / 2011$ & parasitic \\
\hline 10947 & 50 & $\mathrm{LH}_{2}$ & 8921 & VQWK & 20,30 & 64 & $3 / 28 / 2011$ & downstream \\
\hline 10948 & 75 & $\mathrm{LH}_{2}$ & 8921 & VQWK & 20 & 64 & $3 / 28 / 2011$ & (DS) \\
\hline 10951 & 75 & $\mathrm{LH}_{2}$ & $6500-9000$ & VQWK & 30 & 64 & $3 / 29 / 2011$ & downstream QTOR scan \\
\hline 10965 & 150 & $\mathrm{LH}_{2}$ & 8921 & VQWK & 20,30 & 64 & $3 / 30 / 2011$ & \\
\hline 10987 & 150 & $\mathrm{LH}_{2}$ & 8921 & VQWK & 20 & 100 & $3 / 30 / 2011$ & \\
\hline 10988 & 150 & $\mathrm{LH}_{2}$ & 8921 & VQWK & 30,40 & 100 & $3 / 31 / 2011$ & \\
\hline 10991 & 150 & $\mathrm{LH}_{2}$ & 8921 & VQWK & 50 & 100 & $3 / 31 / 2011$ & \\
\hline 11431 & 165 & $\mathrm{LH}_{2}$ & 8921 & VQWK & 45 & 100 & $4 / 15 / 2011$ & \\
\hline 11496 & 165 & $\mathrm{LH}_{2}$ & 6700 & VQWK & 45 & 100 & 4/20/2011 & inelastic \\
\hline 11695 & 26 & $\mathrm{DS} 4 \% \mathrm{Al}$ & 8921 & VQWK & 45 & 100 & $4 / 27 / 2011$ & \\
\hline 11994 & 65 & US $1 \% \mathrm{Al}$ & 8921 & VQWK & 45 & 100 & $5 / 07 / 2011$ & \\
\hline 11995 & 65 & US $1 \% \mathrm{Al}$ & 9054 & VQWK & 45 & 100 & $5 / 07 / 2011$ & \\
\hline
\end{tabular}

Table B.1: Focal plane scanner run list during Q-weak Run I. All the runs used the same threshold setting of $100 \mathrm{mV}$, corresponding to about 3 photo-electrons. 


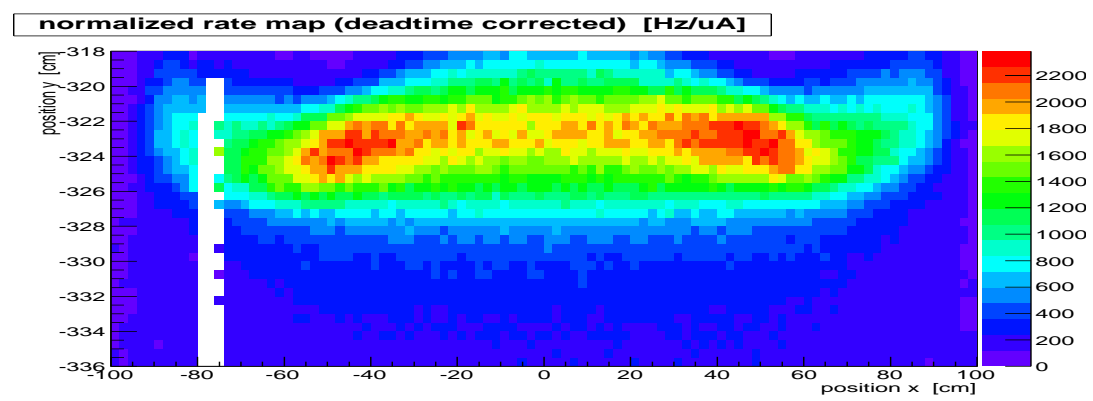

Figure B.1: Normalized scanner rate map at $10 \mu \mathrm{A}$ beam current (run 6615).

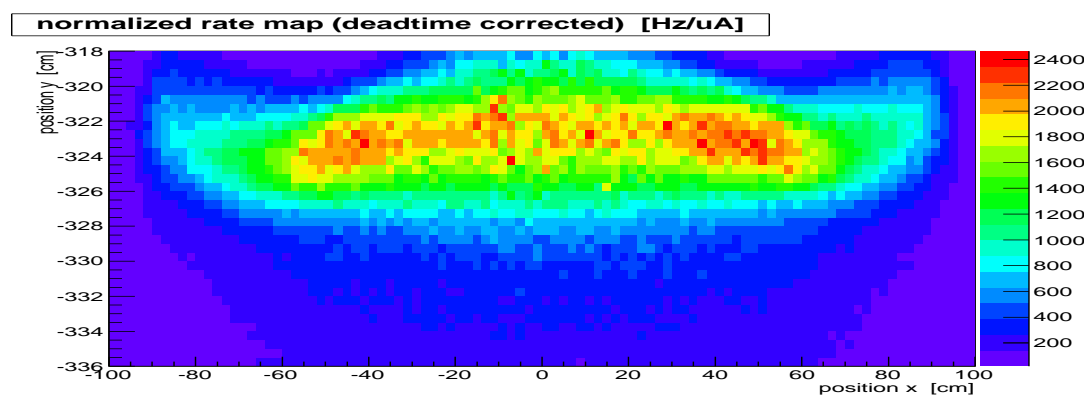

Figure B.2: Normalized scanner rate map at $50 \mu \mathrm{A}$ beam current (run 6616).

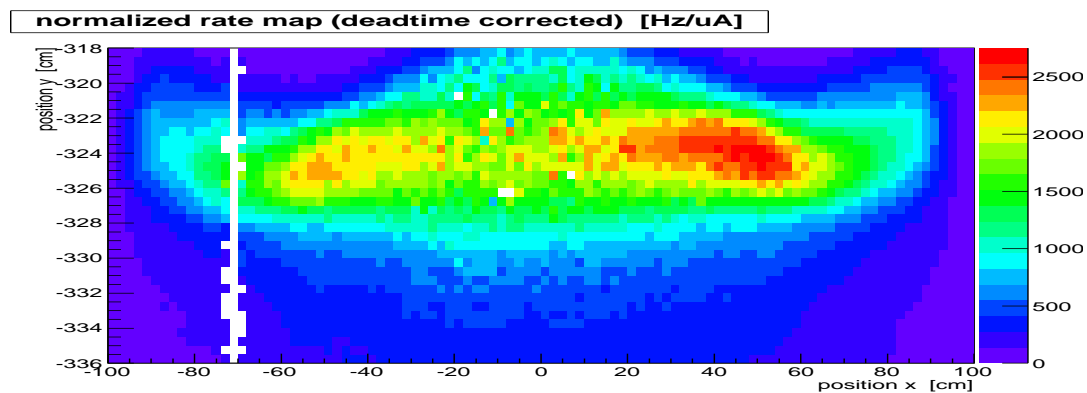

Figure B.3: Normalized scanner rate map at $145 \mu \mathrm{A}$ beam current (run 9710). 


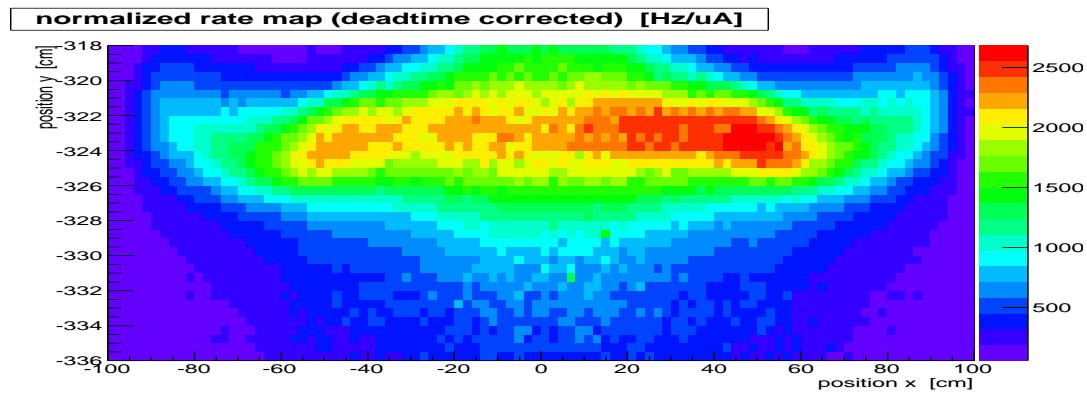

Figure B.4: Normalized scanner rate map at $150 \mu \mathrm{A}$ beam current (run 10044).

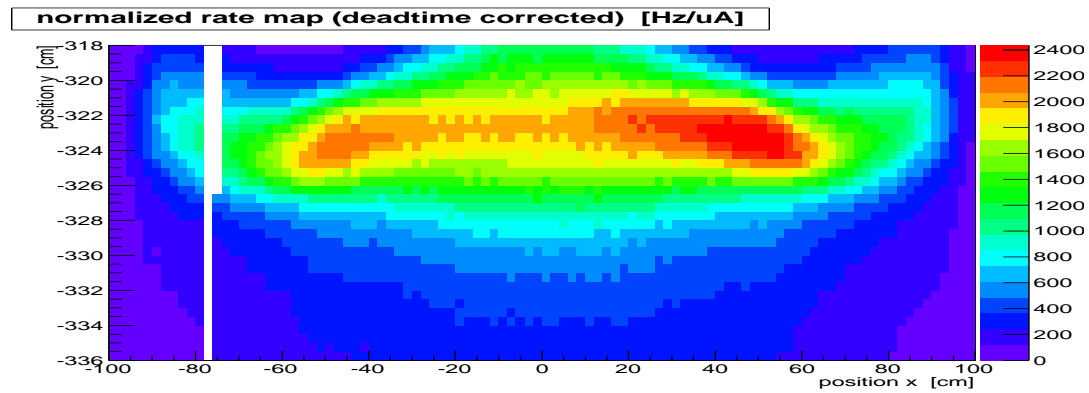

Figure B.5: Normalized scanner rate map at $165 \mu \mathrm{A}$ beam current (run 11431). 


\section{Appendix C}

\section{My Contributions to the Qweak}

\section{Experiment}

My main contributions to the Qweak experiment are briefly summarized in the following list.

1. Scanner detector:

- contributed to the design of the scanner detector,

- constructed the detector and its 2D motion system,

- conducted the detector tests on bench,

- performed the beam tests at TRIUMF and JLab,

- wrote the scanner motion control software,

- installed the detector in the Qweak experiment,

- built up the scanner electronics chain,

- completed the detector commissioning.

2. Main detectors:

- contributed to the construction of main detector optical assemblies. 
3. Track reconstruction:

- wrote one of two major sections of the track reconstruction program - momentum determination by partial track bridging,

- contributed to the Geant4-to-QTR interface code and tested the QTR with Geant4 simulation,

- contributed to coding for other aspects of QTR.

4. DAQ/analysis:

- contributed to coding for multiple aspects of the Qweak analysis software,

- wrote classes for subsystems and electronics modules to decode and process data, such as the scanner class, raster class, scaler class,

- analyzed a subset of tracking and parity data,

- analyzed the full set of scanner data.

5. Monte Carlo simulation:

- wrote an event generator for the Qweak Geant4 simulation package (QweakSimG4), to generate elastic events from liquid hydrogen target, elastic and quasi-elastic events from aluminum target windows,

- wrote and updated geometry description code for the QweakSimG4,

- wrote code to calculate the pre-scattering energy loss,

- wrote Geant4 simulation code to study the scanner detector responses. 


\title{
Appendix D
}

\section{Qweak Collaboration}

\author{
Q-weak Collaboration Spokespersons \\ Carlini, Roger (Principal Investigator) \\ Thomas Jefferson National Accelerator Facility \\ Finn, J. Michael \\ College of William and Mary - (deceased) \\ Kowalski, Stanley \\ Massachusetts Institute of Technology \\ Page, Shelley \\ University of Manitoba \\ Q-weak Collaboration Members \\ Androic, Darko \\ University of Zagreb \\ Armstrong, David \\ College of William and Mary \\ Asaturyan, Arshak \\ Yerevan Physics Institute \\ Averett, Todd \\ College of William and Mary \\ Balewski, Jan \\ Massachusetts Institute of Technology \\ Beaufait, Joseph \\ Thomas Jefferson National Accelerator Facility \\ Beminiwattha, Rakitha \\ Ohio University \\ Benesch, Jay \\ Thomas Jefferson National Accelerator Facility \\ Benmokhtar, Fatiha \\ Christopher Newport University \\ Birchall, James \\ University of Manitoba
}




\begin{tabular}{|c|c|}
\hline Cates, Gordon & University of Virginia \\
\hline Cornejo, Juan & College of William and Mary \\
\hline Covrig, Silviu & Thomas Jefferson National Accelerator Facility \\
\hline Dalton, Mark & University of Virginia \\
\hline Davis, Charles & TRIUMF \\
\hline Deconinck, Wouter & College of William and Mary \\
\hline Deng, Xiaoyan & University of Virginia \\
\hline Diefenbach, Juergen & Hampton University \\
\hline Dow, Karen & Massachusetts Institute of Technology \\
\hline Dunne, James & Mississippi State University \\
\hline Dutta, Dipangkar & Mississippi State University \\
\hline Ent, Rolf & Thomas Jefferson National Accelerator Facility \\
\hline Erler, Jens & University of Mexico \\
\hline Falk, Willie & University of Manitoba \\
\hline Forest, Tony & Idaho State University \\
\hline Franklin, Wilbur & Massachusetts Institute of Technology \\
\hline Furic, Miroslav & University of Zagreb \\
\hline Gaskell, David & Thomas Jefferson National Accelerator Facility \\
\hline Gericke, Michael & University of Manitoba \\
\hline Grames, Joseph & Thomas Jefferson National Accelerator Facility \\
\hline Grimm, Klaus & Louisiana Tech University \\
\hline Hashemi Shabestari, Mitra & Mississippi State University \\
\hline Higinbotham, Douglas & Thomas Jefferson National Accelerator Facility \\
\hline Holtrop, Maurik & University of New Hampshire \\
\hline Hoskins, Joshua & College of William and Mary \\
\hline
\end{tabular}




\begin{tabular}{|c|c|}
\hline Ihloff, Ernie & Massachusetts Institute of Technology \\
\hline Johnston, Kathleen & Louisiana Tech University \\
\hline Jones, Derek & George Washington University \\
\hline Jones, Donald & University of Virginia \\
\hline Jones, Mark & Thomas Jefferson National Accelerator Facility \\
\hline Jones, Richard & University of Connecticut \\
\hline Joo, Kyungseon & University of Connecticut \\
\hline Kargiantoulakis, Emmanouil & University of Virginia \\
\hline Kelsey, James & Massachusetts Institute of Technology \\
\hline Keppel, Cynthia & Hampton University \\
\hline King, Paul & Ohio University \\
\hline Kohl, Michael & Hampton University \\
\hline Korkmaz, Elie & University of Northern British Columbia \\
\hline Leacock, John & Virginia Polytechnic Institute and State University \\
\hline Leckey, John & College of William and Mary \\
\hline Lee, Anna & Virginia Polytechnic Institute and State University \\
\hline Lee, Jeong Han & Ohio University and The College of William and Mary \\
\hline Lee, Lawrence & TRIUMF \\
\hline Lung, Allison & Thomas Jefferson National Accelerator Facility \\
\hline Luwani Zurmbonwi, Ndukum & Mississippi State University \\
\hline MacEwan, Scott & University of Manitoba \\
\hline Mack, David & Thomas Jefferson National Accelerator Facility \\
\hline Magee, Joshua & College of William and Mary \\
\hline Mahurin, Rob & University of Manitoba \\
\hline Mammei, Juliette & Virginia Polytechnic Institute and State University \\
\hline
\end{tabular}




\begin{tabular}{|c|c|}
\hline Martin, Jeffery & University of Winnipeg \\
\hline McHugh, Martin & George Washington University \\
\hline Meekins, David & Thomas Jefferson National Accelerator Facility \\
\hline Mei, Jiawei & Thomas Jefferson National Accelerator Facility \\
\hline Michaels, Robert & Thomas Jefferson National Accelerator Facility \\
\hline Micherdzinska, Anna & George Washington University \\
\hline Mkrtchyan, Arthur & Yerevan Physics Institute \\
\hline Mkrtchyan, Hamlet & Yerevan Physics Institute \\
\hline Munroe, Richard & Christopher Newport University \\
\hline Myers, Katherine & George Washington University \\
\hline Narayan, Amrendra & Mississippi State University \\
\hline Nuruzzaman & Hampton University \\
\hline Opper, Allena & George Washington University \\
\hline Pan, Jie & University of Manitoba \\
\hline Paschke, Kent & University of Virginia \\
\hline Phillips, Sarah & University of New Hampshire \\
\hline Pitt, Mark & Virginia Polytechnic Institute and State University \\
\hline Poelker, B. & Thomas Jefferson National Accelerator Facility \\
\hline Rajotte, Jean-Francois & Massachusetts Institute of Technology \\
\hline Ramsay, W. Desmond & University of Manitoba \\
\hline Ramsey-Musolf, Michael & University of Wisconsin-Madison \\
\hline Roche, Julie & Ohio University \\
\hline Sawatzky, Bradley & Thomas Jefferson National Accelerator Facility \\
\hline Seva, Tomislav & University of Zagreb \\
\hline Silwal, Rupesh & University of Virginia \\
\hline
\end{tabular}


Simicevic, Neven

Smith, Gregory

Smith, Timothy

Solvignon, Patricia

Souder, Paul

Spayde, Damon

Subedi, Adesh

Subedi, Ramesh

Suleiman, Riad

Tsentalovich, Evgeni

Tvaskis, Vladas

van Oers, W.T.H.

Waidyawansa, Buddhini

Wang, Diancheng

Wang, Peiqing

Wells, Steven

Wood, Stephen

Yang, Siyuan

Young, Ross

Zhamkochyan, Simon

Zhao, Zhiwen

Zheng, Xiaochao

Zou, David
Louisiana Tech University

Thomas Jefferson National Accelerator Facility

Dartmouth College

Thomas Jefferson National Accelerator Facility

Syracuse University

Hendrix College

Mississippi State University

George Washington University

Thomas Jefferson National Accelerator Facility

Massachusetts Institute of Technology

University of Manitoba

University of Manitoba

Ohio University

University of Virginia

University of Manitoba

Louisiana Tech University

Thomas Jefferson National Accelerator Facility

College of William and Mary

The University of Adelaide

Yerevan Physics Institute

University of Virginia

University of Virginia

Massachusetts Institute of Technology 


\section{Index}

$u v$ to $x y$ transformation, 109

accidental coincidence, 132

accidental coincidences, 96

Alzak, 86

annihilation operator, 4

asymmetry-based combination, 174

axial form factor, 12

blinding analysis, 170

calibration factor map, 149

CEBAF, 29

charge asymmetry, 32

contrast, 152

counting statistics, 177

covariance matrix, 181

creation operator, 4

cryogenic liquid hydrogen target, 43

DCCT, 164

deadtime, 137

detector resolution, 177

diffuse background, 189

dilution factor, 183 dimensionless normalization, 151

Dirac form factor, 12

Dirac spinor, 11

Electroweak Standard Model, 2

excess noise, 177

fast raster, 42

fiducial area, 65

four-momentum, 9

hadronic form factor, 17

helicity control, 31

helicity operator, 11

helicity quartet pattern, 31

helicity reversal live-time, 177

Insertable Half Wave Plate (IHWP), 32

Lagrangian density, 3

left-right ambiguities, 50

light-weighted $\left\langle Q^{2}\right\rangle, 121$

Linac, 29

linear regression, 180

macro-pulse (MPS), 31 
magnetic shielding, 87

Mott cross-section, 13

Newton iteration, 139

normalized PV asymmetry, 18

operation efficiency, 177

parity-violating asymmetry, 14

parity-violating elastic e-p scattering, 8

partial track parameters, 109

pattern database, 107

pattern matching, 107

Pauli form factor, 12

physics asymmetry, 171

Pockels Cell, 31

post-scattering energy loss, 119

pre-scattering energy loss, 119

Q-weak collaboration, 2

quantum efficiency (QE), 71

quark weak couplings, 5

QweakSimG4, 121

radiation length, 43

radiative correction, 18

rate corrections, 132

rate map, 132

reconstructed momentum, 119

reconstructed scattering angle, 119
Rosenbluth Formula, 13

Sachs form factor, 12

scanner sensitivity matrix, 162

scattering vertex, 119

scintillation yield, 72

self-coincidence trigger, 165

settling time, 31

signal-to-background (S/B) ratio, 71

single photo-electron, 74

singles rates, 134

slug, 178

spin precession, 33

Standard Model (SM), 1

synthetic quartz, 85

time of flight (TOF), 74

toroidal magnet, 46

transimpedance amplifier, 171

transverse polarization, 183

tree level, 5

true coincidence, 132

weak charged current, 6

weak mixing angle, 1, 2

weak neutral current, 4

weighted transformation matrix, 109

Wien filter, 33

yield-based combination, 174 